
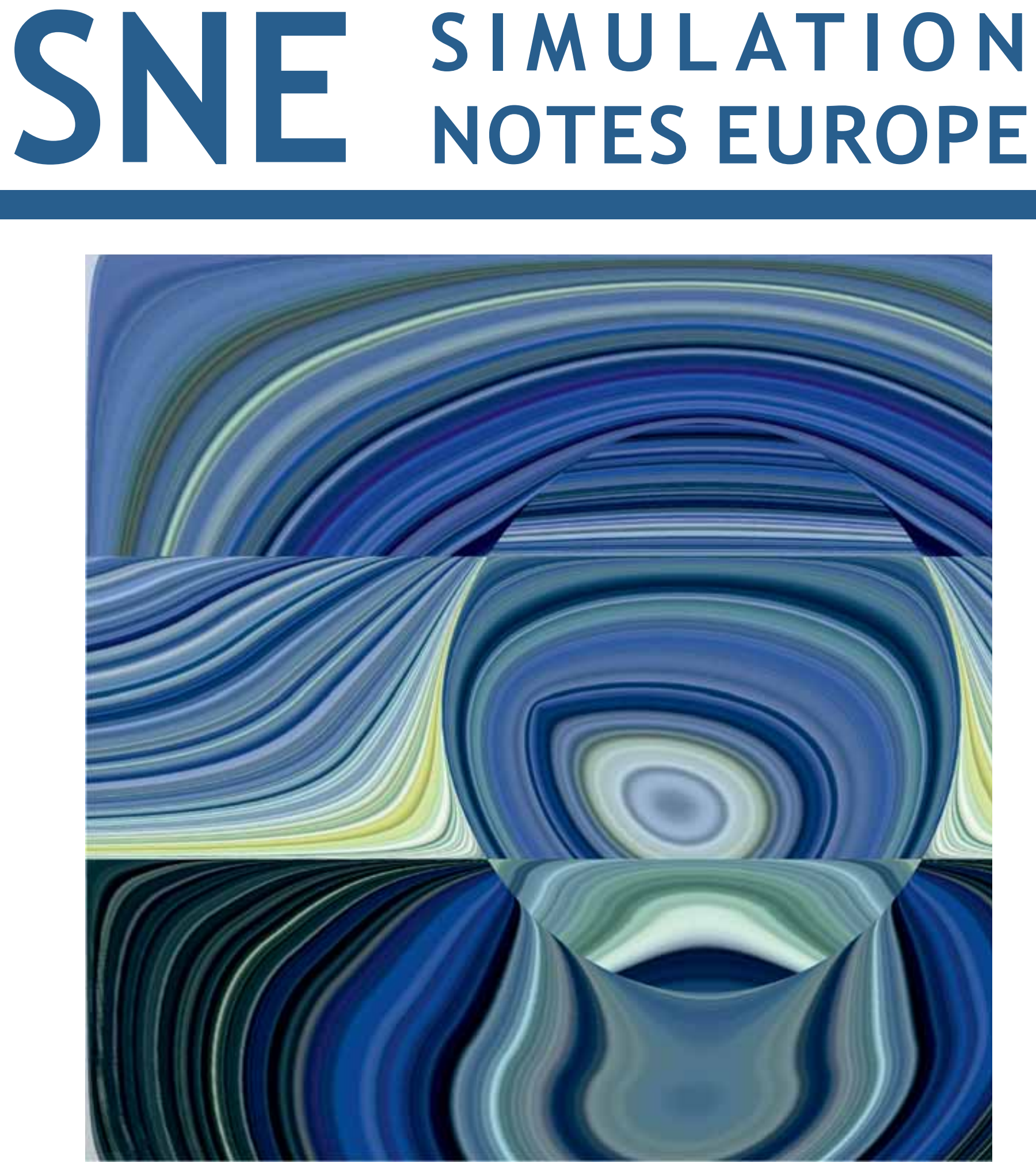

Volume 25 No.3-4 December 2015

doi: 10.11128/sne.25.34.1030

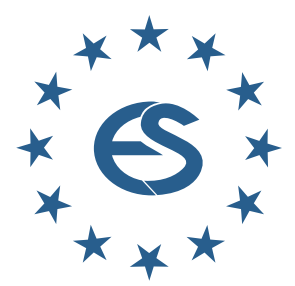

Journal on Developments and Trends in Modelling and Simulation Membership Journal for Simulation Societies and Groups in EUROSIM
Print ISSN 2305-9974

Online ISSN 2306-0271

ARGESIM 


\section{A modern approach to modeling and simulation}

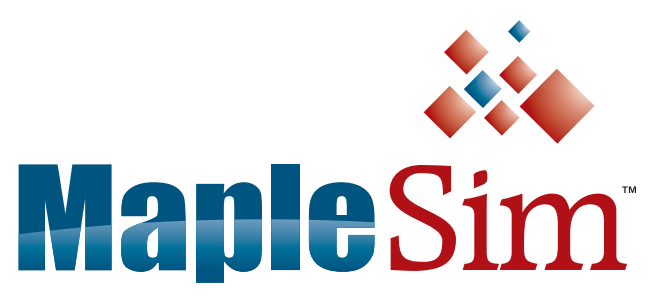

With MapleSim, educators have an industry-proven tool to help bridge the gap between theory and practice.

- MapleSim illustrates concepts, and helps students learn the connection between theory and physical behavior

- A wide variety of models are available to help get started right away
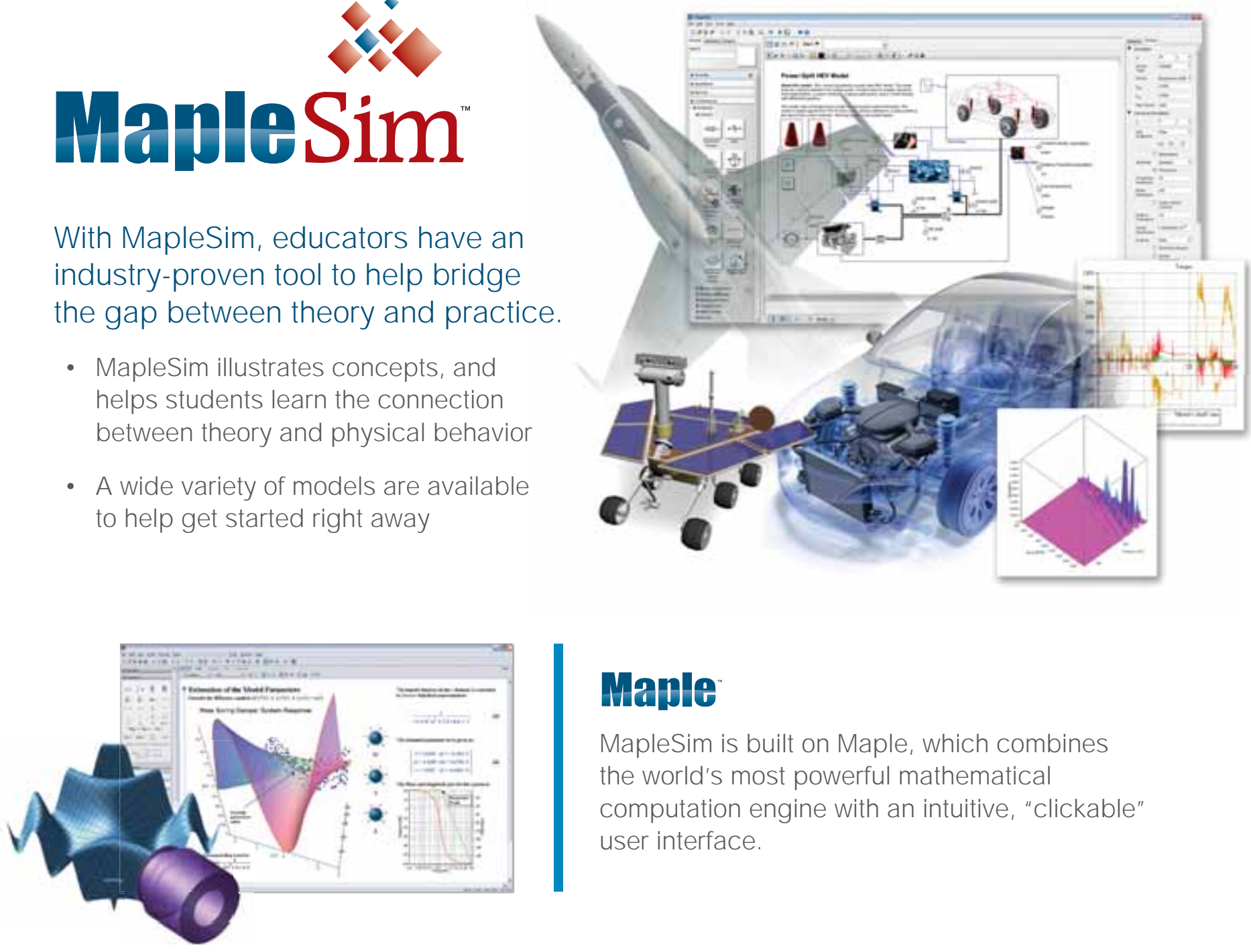

\section{Maple}

MapleSim is built on Maple, which combines the world's most powerful mathematical computation engine with an intuitive, "clickable" user interface.

To learn more about how you can reinforce engineering concepts using a combination of theory, simulation, and hardware, view this webinar.

\section{www.maplesoft.com/SNEWebinar}

Contact us: $+49(0) 241 / 980919-30$ 


\section{Editorial}

Dear Readers - This last issue of SNE Volume 25 reflects the broad variety of nowadays modeling and simulation, looking ahead for enrichment of SNE's publications with computational complex systems, starting with SNE Volume 26, 2016. The first part of contributions deal with modelling and simulation in physiology, whereby an overview note by $G$ Wedemann presents a computer simulation for cancer spread and treatment. The second part of contributions range from classic mechanic area and Modelica development and application via simulation for ambient assisted living to stochastic PDE modelling for epidemics.

Thanks to Vlatko Ceric, past president

of CROSSIM, who provided a series of his algorithmic art as cover pages SNE Volume 25 (samples at right).

'Algorithms, mathematics and art are interrelated in an art form called algorithmic art. Algorithmic art is visual art generated by algorithms that completely describe creation of images.'
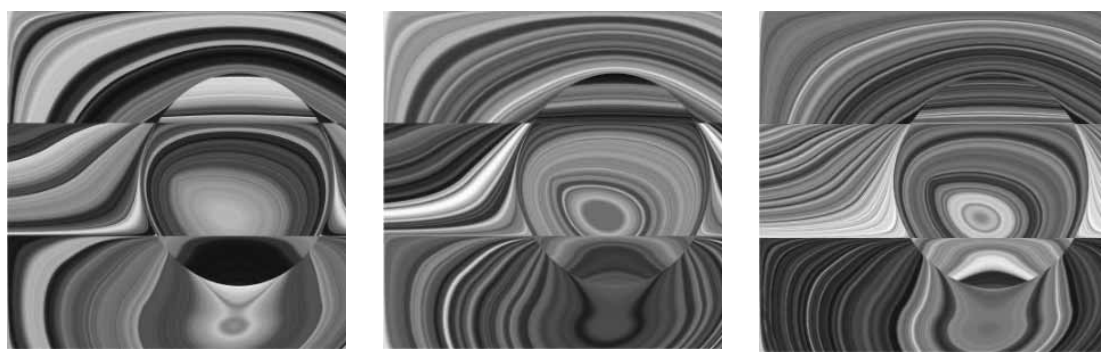

I would like to thank all authors for their contributions, and the organizers of the EUROSIM conferences for co-operation in post-conference publication, and the ARGESIM SNE staff for helping to manage the SNE administration and the improved SNE layout and extended templates for submissions (now also tex).

Felix Breitenecker, SNE Editor-in-Chief, eic@sne-journal.org; felix.breitenecker@tuwien.ac.at

\section{Contents SNE 25(3-4)}

SNE doi: $10.11128 /$ sne.25.34.1030

Computer Simulation of the Metastatic Progression and Treatment Intervention.

B. Hoffmann, A. Bethge, U. Schumacher, G. Wedemann ...... 123 Implementation, Testing, and Evaluation of Center Selection Methods for Supervised Radial Basis Networks to Enhance Breast Cancer Analysis. M. Nanavati, B. R. Bracio

Evaluation of Risk Factors for Parametrization of Cancer

Models. A. Bauer, G. Zauner, C. Urach

Nonlinear Methods in Heart Rate Variability: Can they Distinguish between Nonpathological and Pathological Subjects?

S. Hagmair, M. Bachler, S. Wassertheurer, C. Mayer

Reservoir Wave Paradigm: An Implementation and Sensitivity

Analysis. M.Ebner, B. Hametner, S. Parragh, S. Wassertheurer .. 151

Learning the Structure of Graphical Models Based on

Discrete Time Series Data in the Context of Ambient

Assisted Living. S. Fallmann, J. Kropf

Modeling, Simulation and Control of a Loading Bridge.

M. Hancic, G. Karer, I. Škrjanc

Cloud Computing Applied to Calibration of Flood Simulation

Models. M. Brettschneider, B. Pfützner, F. Fuchs-Kittowski

Decision Making in Ambient Assisted Living Environments

based on Uncertain and Fuzzy Data. D. Brunmeir, J. Kropf .... 179

Expressing Requirements in Modelica. L. Buffoni, P. Fritzson ... 185

PDE Modeling with Modelica via FMI Import of HiFlow3 C++

Components with Parallel Multi-core Simulations.

K. Stavåker, P. Fritzson, C. Song, M. Wlotzka, V. Heuveline

Probabilistic Drift Formulation of SIRS Models based on

SPDEs and the Kolmogorov Equation. G. Schneckenreither

ARGESIM Benchmark C13 'Crane and Embedded Control':

SIMULINK-modelled Dynamics and MATLAB-programmed

Control. G. Janisch, M. Leopoldseder, A. Körner

EUROSIM Societies Info \& News N1-N8

\section{Reader's Info}

Simulation Notes Europe publishes peer reviewed Technical Notes, Short Notes and Overview Notes on developments and trends in modelling and simulation in various areas and in application and theory, with main topics being simulation aspects and interdisciplinarity.

Individual submissions of scientific papers are welcome, as well as post-conference publications of contributions from conferences of EUROSIM societies. Furthermore SNE documents the ARGESIM Benchmarks on Modelling Approaches and Simulation Implementations with publication of solutions (Benchmark Notes). Special Educational Notes present the use of modelling and simulation in education and for e-learning.

SNE is the official membership journal of EUROSIM, the Federation of European Simulation Societies. A News Section in SNE provides information for EUROSIM Simulation Societies and Simulation Groups.

SNE is published in a printed version (Print ISSN 23059974) and in an online version (Online ISSN 2306-0271). With Online SNE the publisher ARGESIM follows the Open Access strategy, allowing download of published contributions for free. Since 2012 Online SNE contributions are identified by a DOI (Digital Object Identifier) assigned to the publisher ARGESIM (DOI prefix 10.11128). Print SNE, high-resolution Online SNE, full SNE Archive, and source codes of the Benchmark Notes are available for members of EUROSIM societies.

\section{SNE Print ISSN 2305-9974, SNE Online ISSN 2306-0271}

SNE Issue 25(34) Dec. 2015 doi: 10.11128/sne.25.34.1030

$\rightarrow$ www.sne-journal.org

莑=- office@sne-journal.org, eic@sne-journal

$\triangle$ SNE Editorial Office, c/o ARGESIM / DWH,

Neustiftgasse 57-59, 1070 Vienna, Austria 


\section{SNE Editorial Board}

SNE - Simulation Notes Europe is advised and supervised by an international scientific editorial board. This board is taking care on peer reviewing and handling of Technical Notes, Education Notes, Short Notes, Software Notes, Overview Notes, and of Benchmark Notes (definitions and solutions). At present, the board is increasing (see website):

David Al-Dabass, david.al-dabass@ntu.ac.uk Nottingham Trent University, UK

Felix Breitenecker, Felix.Breitenecker@tuwien.ac.at Vienna Univ. of Technology, Austria, Editor-in-chief

Maja Atanasijevic-Kunc,maja.atanasijevic@fe.uni-lj.si Univ. of Ljubljana, Lab. Modelling \& Control, Slovenia

Aleš Belič, ales.belic@sandoz.com Sandoz / National Inst.f. Chemistry, Slovenia

Peter Breedveld, P.C.Breedveld@el.utwente.nl University of Twenty, Netherlands

Agostino Bruzzone, agostino@itim.unige.it Universita degli Studi di Genova, Italy

Francois Cellier, fcellier@inf.ethz.ch ETH Zurich, Switzerland

Vlatko Čerić,vceric@efzg.hr Univ. Zagreb, Croatia

Russell Cheng,rchc@maths.soton.ac.uk University of Southampton, UK

Eric Dahlquist, erik.dahlquist@mdh.se, Mälardalen Univ., Sweden

Horst Ecker,Horst.Ecker@tuwien.ac.at Vienna Univ. of Technology, Inst. f. Mechanics, Austria

Vadim Engelson, vadim.engelson@mathcore.com MathCore Engineering, Linköping, Sweden

Edmond Hajrizi, ehajrizi@ubt-uni.net

University for Business and Technology, Pristina, Kosovo

András Jávor,javor@eik.bme.hu,

Budapest Univ. of Technology and Economics, Hungary

EskoJuuso,esko.juuso@oulu.fi

Univ. Oulu, Dept. Process/Environmental Eng., Finland

Kaj Juslin, kaj.juslin@vtt.fi

VTT Technical Research Centre of Finland, Finland

Andreas Körner, andreas.koerner@tuwien.ac.at

Technical Univ. Vienna, E-Learning Dpt., Vienna, Austria

Francesco Longo,f.longo@unical.it

Univ. of Calabria, Mechanical Department, Italy

Yuri Merkuryev,merkur@itl.rtu.lv, Riga Technical Univ.

David Murray-Smith,d.murray-smith@elec.gla.ac.uk

University of Glasgow, Fac. Electrical Engineering, UK

Gasper Music,gasper.music@fe.uni-lj.si

Univ. of Ljubljana, Fac. Electrical Engineering, Slovenia

Thorsten Pawletta,pawel@mb.hs-wismar.de

Univ. Wismar, Dept. Comp. Engineering, Wismar, Germany

Niki Popper,niki.popper@dwh.at dwh Simulation Services, Vienna, Austria

Kozeta Sevrani, kozeta.sevrani@unitir.edu.al Univ. Tirana, Inst.f. Statistics, Albania

Thomas Schriber, schriber@umich.edu University of Michigan, Business School, USA

Yuri Senichenkov, sneyb@dcn.infos.ru

St. Petersburg Technical University, Russia
Siegfried Wassertheurer, Siegfried.Wassertheurer@ait.ac.at AIT Austrian Inst. of Technology, Vienna, Austria

Sigrid Wenzel, S.Wenzel@uni-kassel.de

Univ. Kassel, Inst. f. Production Technique, Germany

\section{Author's Info}

Authors are invited to submit contributions which have not been published and have not being considered for publication elsewhere to the SNE Editorial Office. Furthermore, SNE invites organizers of EUROSIM conferences to submit postconference publication for the authors of their conferences.

SNE distinguishes different types of contributions (Notes):

- Overview Note - State-of-the-Art report in a specific area, up to 14 pages, only upon invitation

- Technical Note - scientific publication on specific topic in modelling and simulation, $6-8$ pages

- Education Note - modelling and simulation in / for education and e-learning; 6 - 8 pages

- Short Note - recent development on specific topic, max. 4 p.

- Software Note - specific implementation with scientific analysis, max 4 pages

- Benchmark Note - Solution to an ARGESIM Benchmark; commented solution 4 pages, comparative solutions 4-8 pages

Further info and templates (doc, tex) at SNE's website.

\section{SNE Contact \& Info}

$\rightarrow$ www.sne-journal.org

琵- office@sne-journal.org, eic@sne-journal.og

$\triangle$ SNE Editorial Office, ARGESIM / dwh Simulation Services, Neustiftgasse 57-59, 1070 Vienna, Austria

\section{SNE SiM ULATION NOTES EUROPE}

ISSN SNE Print ISSN 2305-9974, SNE Online ISSN 2306-0271

WEB: $\rightarrow$ www.sne-journal.org, DOI prefix 10.11128/sne

Scope: Technical Notes, Short Notes and Overview Notes on developments and trends in modelling and simulation in various areas and in application and theory; benchmarks and benchmark documentations of ARGESIM Benchmarks on modelling approaches and simulation implementations; modelling and simulation in and for education, simulation-based e-learning; society information and membership information for EUROSIM members (Federation of European Simulation Societies and Groups).

Editor-in-Chief: Felix Breitenecker, Vienna Univ. of Technology, Inst. f. Analysis and Scientific Computing, Div., Math. Modelling and Simulation, Wiedner Hauptstrasse 8-10, 1040 Vienna, Austria; 玤” Felix.Breitenecker@tuwien.ac.at, 㭋- eic@sne-journal.org

Layout / Administration: J. Tanzler, F. Preyser, T. Vobruba; C. Wytrzens, R. Leskovar et al.; M ath. M odelling and Simulation Group, Vienna Univ. of Technology, Wiedner Haupstrstasse 8-10, 1040 Vienna , 夆=0 office@sne-journal.org

Print SNE: Grafisches Zentrum, TU Vienna, Wiedner Hauptstrasse 8-10, 1040, Vienna, Austria

Online SNE: ARGESIM / ASIM , c.o. dwh Simulation Services, Neustiftgasse 57-59, 1070 Vienna, Austria

Publisher: ARGESIM ARBEITSGEM EINSCHAft SIMULATION NEWS - WORKING COM MITTEE SIM ULATION NEWS, Neustiftgasse 57-59, 1070 Vienna, Austria; $\rightarrow$ www.argesim.org, on behalf of ASIM ( $\rightarrow$ www.asim-gi.org and EUROSIM $\rightarrow$ www.eurosim.info (c) ARGESIM / EUROSIM / ASIM 2015 


\title{
Computer Simulation of the Metastatic Progression and Treatment I ntervention
}

\author{
Bertin Hoffmann ${ }^{1}$, Anja Bethge ${ }^{1}$, Udo Schumacher ${ }^{2}$, Gero Wedemann ${ }^{1 *}$ \\ ${ }^{1}$ Competence Center Bioinformatics, Institute for Applied Computer Science, University of Applied Sciences Stralsund, \\ Zur Schwedenschanze 15, 18435 Stralsund, Germany; *gero.wedemann@fh-stralsund.de \\ Institute of Anatomy and Experimental Morphology, University Cancer Center, University Medical Center Hamburg, \\ Martinistrasse 52, 20246 Hamburg, Germany
}

Simulation Notes Europe SNE 25(3-4), 2015, 123 - 131

DOI: $10.11128 /$ sne.25.on.10301

Received: Draft Sept. 15, 2015 (Inv. Lecture ASIM STS 2015);

Revised: December 10, 2015, Accepted: December 20, 2015;

\begin{abstract}
In many cancer entities, the primary tumour is able to spread metastases to distant sites in our body. In the majority of cases the fatality is not caused by the primary tumour but the metastases. Despite intensive research in the last decades, many details of this process are still not understood. Our collaboration developed a computer model that enables quantitative investigations of the metastatic progression. Different models of metastatic progression were compared with clinical and experimental data to gain new insights into this process.

The computer model is based on a discrete event simulation. Analytical functions describe the growth of the primary tumour and the metastases whereas intravasation event models the invasion of cancer cells from the primary tumour into the bloodstream. Further events simulate the behaviour of this cell until it either dies or founds a new metastasis. Clinical and experimental data were analysed to investigate, whether metastases spread early or late during the course of the disease, if metastases themselves are also able to metastasize and the impact of the immune system on the process of metastasis formation.

Furthermore, different therapies such as resection of the primary tumour, chemotherapy or radiotherapy and its impact on the number of metastases and the tumour burden were simulated.
\end{abstract}

\section{I ntroduction}

In 2013, 223842 citizens of Germany died because of cancer. Consequently, cancer is the second most common cause of death in Germany [1]. The same holds for many other developed countries. In general, not the primary tumour is the cause of death, but in more than $90 \%$ of the cases it is the distant metastases. For the development of new treatments, it is very important to understand the process of metastatic progression in detail to identify steps, which should be the target of pharmacological intervention.

The complex process of metastatic progression consists of many consecutive steps, which are influenced by the surrounding tissue and the immune system. When the primary tumour reaches a certain tumour mass, the oxygen and nutrient supply reduces over the time. Hence, the primary tumour sends angiogenic signals for neoformation of blood vessels. Following this process, cells of the primary tumour are able to invade blood or lymphatic vessels through the basal membrane, a process that is termed intravasation. Due to intravasation, malignant cells can now move to distant organs e.g. lung or liver and are called circulating tumour cells (CTCs). During the transport through the blood vessels, $99.9 \%$ of the malignant cells undergo apoptosis or are eliminated by the immune system [2]. Some of the few remaining cells are able to leave the blood vessels and migrate into the connective tissue spaces of the colonised organ. The isolated tumour cells are called disseminated tumour cells (DTCs) and are their origin of new (micro)metastases after they have been stimulated to grow. 
A micrometastasis can send angiogenic signals like the primary tumour and grow up to become a macrometastasis, if it survives [2, 3]. This whole process is called metastatic cascade, because every stage has to be complete before the next stage starts.

The metastatic process is subject of many current experimental and clinical research projects. Different mathematical models have been developed and data generated by these models were compared with clinical and experimental data to obtain new insights about this process. Common mathematical models usually focus on parts of the metastatic process e.g. molecular characteristics of a single tumour cell or the process of tumour growth. The application of these models enables for example the determination of the optimal dose for radiation therapy [4] or the development of better therapeutic strategies [5-7].

The scope of pure mathematical models is very limited in comparison with computer models which can model complex settings more easily. In contrast to pure mathematical models, computer models are also able to deliver conclusions about the range of variation of the results of the computations.

This article summarizes a computer model, which was used by our groups to analyse clinical and experimental data and to investigate alternatively treatments [8-11].

\section{Methods}

\subsection{Compartments and events}

A simulated system consists of a collection of components. These components have different properties and may be related with each other [12]. A component can be e.g. the primary tumour, which is related with the component bloodstream, because the tumour cells that form the primary tumour are able to intravasate into the bloodstream. Both components have the property 'number of cells'. Changes of the state of a component can be described as events, e.g. changes of properties or relation between two or more components. Our computer model comprises compartments and events (see Figure 1).

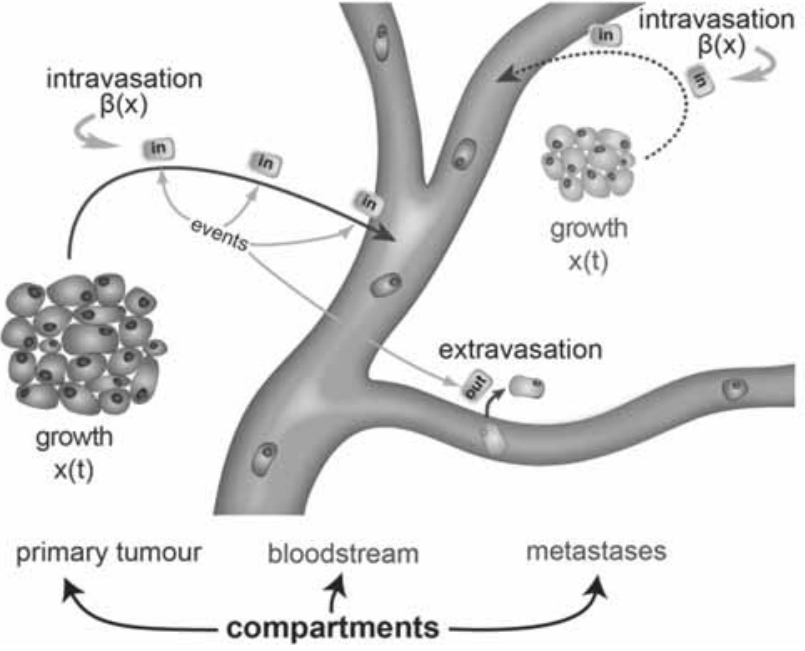

Figure 1. Schematic illustration of compartments and events. Source: [9].

In the computer model compartments represent components such as the primary tumour, the bloodstream and metastases. These compartments will be modelled continuously or discretely. By modelling a compartment as a continuous compartment, the behaviour can be described with mathematical functions. A growth function models the growth behaviour of a compartment and a colonization rate describes the spreading behaviour of malignant cells into other compartments such as the bloodstream. In contrast, the behaviour of discrete compartments is modelled by events.

For every type of discrete compartments a set of possible event types is defined with a possibility of its occurrence. During the creation of an event action, an event will be randomly chosen from this list accordingly to its probability of occurrence.

Events describe which action is triggered at a specific time [8-10]. These events are stored in an events list sorted by the time of their occurrence. One or more compartments will be modified during the execution of an event.

Furthermore, events can be created during an execution of other events, e.g. after an intravasation event of a malignant cell from the primary tumour into the bloodstream, a new event will be created and described the new behaviour or rather the next step of this cell. 
Events can be local and global events. Local events are limited to modify only one or two compartments, whereas global events are able to modify all compartments. Local events describe e.g. cell division or cell death (apoptosis). Global events are typically used to describe treatments such as chemotherapy, since this treatment affects not only the primary tumour, but also metastases.

In the discrete event simulation the current state of the system is modified at specific points in time. The number of tumour cells in the primary tumour can be decreased by cell death (apoptosis) or increased by cell division or intravasation of a single malignant cell of the primary tumour into the bloodstream. For example, the event 'cell division' increases the number of cells in the concerned compartment. Afterwards, two new events are generated which describe the behaviour of the mother cell and their daughter cell, e.g. apoptosis or intravasation. The event 'Apoptosis' decreases the number of cells in the compartment. The 'Intravasation' event decreases the number of cells in the compartment and increases the number of cells in the following compartment (e.g. bloodstream). A newly generated event describes the behaviour of this cell in the bloodstream, e.g. apoptosis or extravasation. The time point of this new event is generated using a Gaussian or Uniform distribution. The computation for each event will be performed separately.

In computer model described here, a continuous compartment will be used to model the growth of the primary tumour and the formation of metastases, the bloodstream is modelled by a discrete compartment (see Figure 1).

\subsection{Tumour growth in continuous compartments}

The number of cells $x$ in a continuous compartment changes over time with the rate $g(x)$ :

$$
\frac{d x}{d t}=g(x), \quad N_{0}:=x(t=0)
$$

In a patient the tumour growth starts with a single cell $N_{0}=1$ [13]. In experiments with model organisms e.g. mice with injected tumour cells at the beginning the number of cells at the time $t=0$ can be larger than 1 .
For the growth rate $g(x)$, many functions are available, e.g. linear, exponential, logarithmic or power law functions. The most common function that describe the growth behaviour is the Gompertz function [13]. This function is a sigmoid function and is defined as

$$
g(x)=a x \ln (b / x)
$$

where $a$ is the growth constant and $b$ the maximum size of the tumour [9]. Integrating Eq. (2) in Eq. (1), the number of tumour cells at time $t$ is given by

$$
x(t)=b\left(\frac{b}{N_{0}}\right)^{-e^{-a t}} .
$$

\subsection{I ntravasation in continuous compartments}

In our computer model, the event 'Intravasation' comprises all steps that are necessary to model the migration of a malignant cell from the primary tumour into the bloodstream. The intravasation event begins with the individual tumour cell braking away from the primary tumour and degrading the basal membrane with subsequent invasion of the surrounding connective tissue. Some cells succeed to detach from this tissue and invade into the bloodstream. The intravasation event is described by the colonization rate

$$
\beta(x)=m x^{\delta / 3},
$$

where $m$ is the colonization coefficient and $\delta$ is the fractal dimension, i.e. how well is the tumour supplied with blood [13].

The next time point to execute an intravasation event will be computed by the help of a numerical integration ensuing from the last executional time point. For this purpose a random number between 0 and 2 will be picked. The colonization rate will be numerically integrated in time until it reaches this random number. This time point will be selected for the next intravasation event [8].

\subsection{Modelling of treatments}

Our computer model provides the possibility to include different treatments such as resection of the primary tumour, chemotherapy, external beam radiation, radio embolization and radio immunotherapy into the simulation. As an example we describe the resection of the primary tumour. Other treatments are described in detail in [10]. 
By performing a resection of the primary tumour in a discrete compartment, the number of cells will be set to 0 . Events belonging to the primary tumour will be deleted from the event list. In a continuous compartment the growth function will be replaced by $x(t)=0$. Consequently, the colonization rate gives $\beta(x)=0$, i.e. the primary tumour cannot longer spread malignant cells.

\subsection{Modelling the dormancy state}

After the extravasation of a malignant cell from the bloodstream into the surrounding tissue, some tumour cells can switch to a dormant state. In this situation, the number of cells of the affected compartment remains constant for a time span.

After a few cell division cycles from the single cell a multi-cellular cluster evolved. In some cases, the development of the cluster slows down for a limited time, so called late dormancy, and increases afterwards. The reason for this behaviour could be the beginning of angiogenesis or the different regulation of gene expression of the cells within the metastases.

Discrete compartments do not generate events concerning the growth behaviour of the primary tumour during a dormant state. After the end of the dormant state an event will be executed to reset this state to the previous state. E.g. the primary tumour will continue growing with the appropriate parameters which were valid before the dormant state had occurred.

In a continuous compartment, the current growth function will be replaced with a constant function during dormancy. Afterwards, the tumour or metastasis will resume growing with the configured parameters. During late dormancy the size of the compartment is constant and continues growing after the dormancy.

\subsection{Simulation}

Our computer model can be configurated using an XML-File (Extensible Markup Language [14]). The simulated system can assembled like building blocks to define the component (e.g. primary tumour, metastases or bloodstream) and their properties (e.g. growth rate or colonization rate). A XML schema defines the structure of this XML file. Many samples and the description of the schema itself are public available under [15].
At the start of the simulation, the software reads the XML file and generates all necessary compartments and already known events (e.g. treatment intervention at day $\mathrm{X})$. The current state of the simulation progress will be stored in a file in Microsoft Excel format periodically. This file contains, amongst other data, current time, current size of the primary tumour and current number of cells of the metastases [10].

Since the generation of events is based on random numbers (see Section 1.3), each simulation run yields different results. Therefore, a simulation scenario will be simulated typically 100 times to obtain sufficient variance of the data. Finally, the mean value is determined from these 100 simulation runs [10].

\subsection{Configuration of the simulation}

A scenario with resection of the primary tumour and chemotherapy serves as an example for the configuration of a simulation (see Listing 1 in the Appendix).

A three-cycle chemotherapy starts on day 850 with an interval of 50 days (850, 900 and 950). The primary tumour will be resected on day 1100 . Size of the primary tumour and number of cells of the metastases are illustrated in Figure 2.

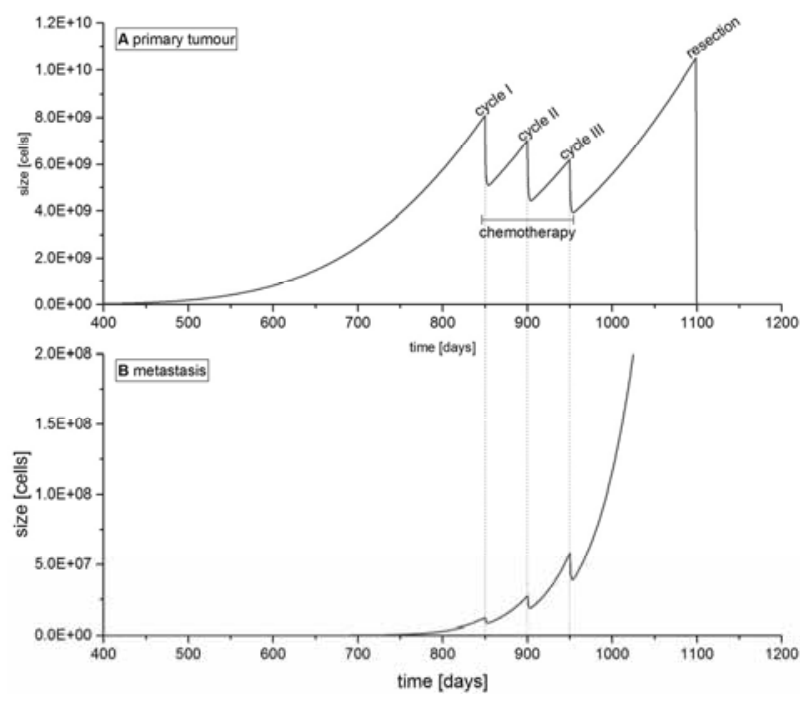

Figure 2. Impact of chemotherapy and resection of the primary tumour on the size of the primary tumour $(A)$ and the number of cells in all metastases (B). 
As shown in Figure 2A, chemotherapy influences the size of the primary tumour just for a short time. Only the resection reduces the size of the primary tumour to 0 . Regarding the number of cells in all metastases, chemotherapy has also an influence for only a limited period (see Figure 2B).

\section{Clinical Relevance of Late Spread Metastases}

\subsection{Research question}

Based on clinical data of an untreated patient with hepatocellular carcinoma (HCC) and multiple metastases, among others, the following questions were examined:

1. Are metastases able to spread metastases on their own?

2. Are cells, which were spread from large tumours, still able to form metastases?

\subsection{Simulated scenarios}

To examine these questions, four different scenarios were simulated (Table 1). In scenario A both the primary tumour and the metastases can spread new metastases. In scenario B just the primary tumour is able to do so. Scenario C and D model the case where late spread tumour cells of large tumours or metastases are not able to form new metastases.

For the simulation three values were chosen in relation to the maximum size $b$ for the size of the tumour, where the disseminated cells cannot form metastases any longer: $10^{8}, 10^{9}$ and $10^{10}$ cells. In Scenario D, such as in scenario B, metastases are not able to spread metastases on their own.

\begin{tabular}{lll}
\hline late & metastases are & metastases are \\
disseminated & able to & not able to \\
tumour cells... & metastasize & metastasize
\end{tabular}

\begin{tabular}{lll}
\hline $\begin{array}{l}\text { are able to } \\
\text { metastasize }\end{array}$ & A & B \\
\hline $\begin{array}{l}\text { are not able to } \\
\text { metastasize }\end{array}$ & C & D \\
\hline
\end{tabular}

Table 1. Simulated scenarios. Source: [9].

\subsection{Results}

The results are shown in Figure 3. The diagrams show the cumulated quantity of metastases in relation to the size. Scenarios A and B did not differentiate in the range of the clinical data (squares, circles and triangles), which were determined on days 1110, 1237 and 1310 after the estimated origin of the primary tumour through CT-Scans. Only in the range of small metastases $\left(<10^{6}\right.$ cells) differences on day 1310 were recognizable.

This is because metastases must reach a certain size to be able to form metastases on their own. Therefore, the CT-Scans can apparently detect metastases spread by the primary tumour only. Only in the later process (day 1310) metastases from metastases could develop.

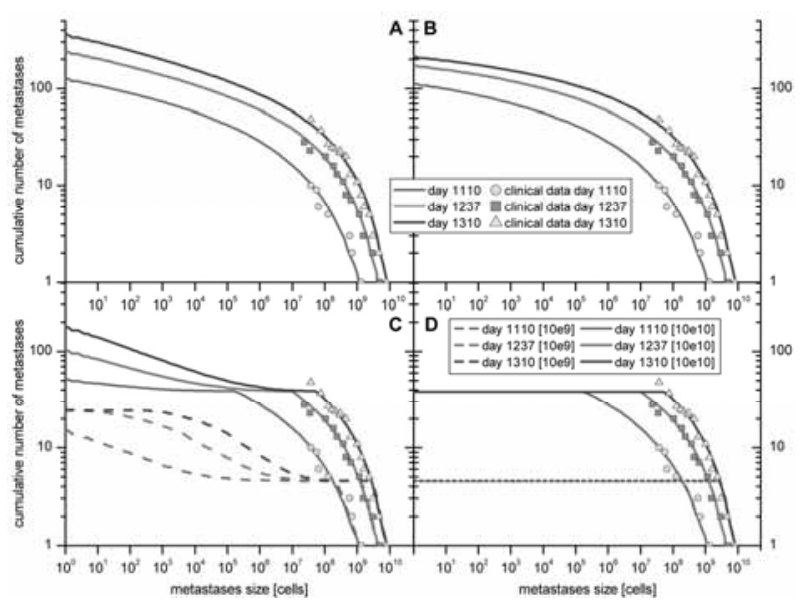

Figure 3. Results of the simulated scenarios A-D. The circles, squares and triangles represent the clinical data on different days.

From the available data it is not possible to make a reliable statement, if metastases can form metastases on their own. Data from more detailed investigations would be needed to answer this question. However, it turned out that in the range of these scenarios the question is not of clinical relevance, since metastases derived from the primary tumour alone contribute enough cells to the fatal tumour burden.

To answer the question whether large tumours or metastases are still spreading malignant cells, which are able to form metastases, scenario $\mathrm{C}$ and $\mathrm{D}$ were examined. Therefore, different sizes were chosen as limit values, whereby the primary tumour or the metastases spread cells are not able to build metastases anymore. 
At a limit value of $10^{9}$ cells it is recognizable that the data from the simulations (dotted line in C and D) did not match the clinical data (circles, squares and triangle). Thus cells, which are spread from a primary tumour or metastases that are bigger as $10^{9}$ cells, are able to form metastases. The clinical data and the simulation results are close together at the size of $10^{10}$ cells. The clinical data does not cover the whole range of size of the metastases. Therefore the question can also not be answered unequivocally $[8,10]$. However, it turns out even here, that the question is not of clinical relevance for the analysed scenarios.

\section{Metastatic Progression under the I nfluence of Treatments}

\subsection{Research question}

The influence of different treatments on the progression of metastasis formation was investigated with the aid of the computer model. The relevance of an early diagnosis on the therapy result was evaluated.

\subsection{Simulated scenarios}

The study was performed on the basis of the clinical data of a patient with HCC as in Section 2. The following treatments were simulated [15]: resection of the primary tumour, cycle specific and cycle non-specific chemotherapy, external beam radiation, radio immunotherapy, radio embolization and a combination of resection and a cycle specific chemo therapy. The complete parameterization of the examined scenarios is shown in Table 1 in [15]. In all scenarios the therapy start is simulated for day 700 (early diagnosis) and for day 1500 (late diagnosis). Scenarios were considered where the metastases had the ability to form metastases themselves or not.

\subsection{Results}

As an example, the influence of the resection of the primary tumour will be discussed here.

In the case of an early diagnosis and the subsequent early therapy (resection), there is a temporarily stagnation on the formation of new metastases if metastases are able to metastasize (see Figure 4A) or rather a complete stagnation else (see Figure 4B).

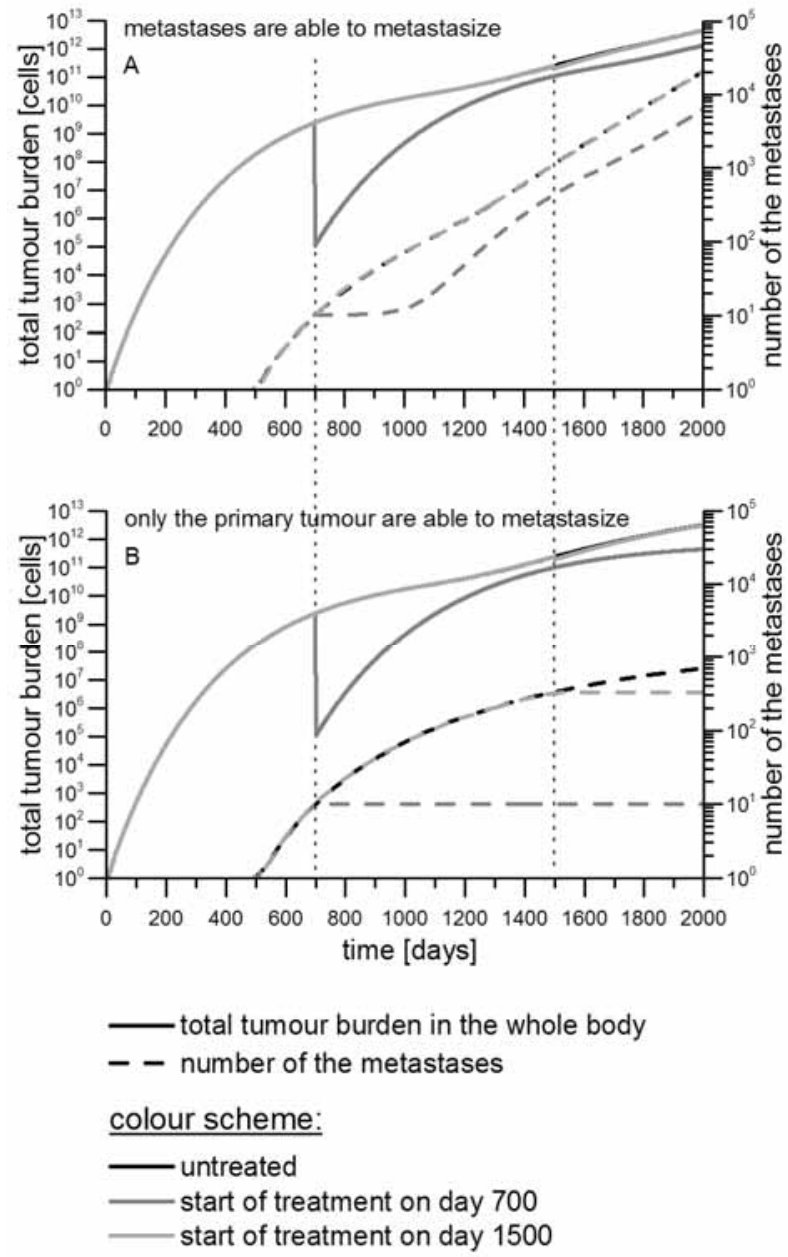

Figure 4. Results of the resection of the primary tumour on tumour burden and number of metastases on day 700 . In scenario $A$, the primary tumour and the metastases are able to spread malignant cells. In scenario $B$, only the primary tumour is able to do so. Adapted from: [16].

In the case that metastases can form metastases on their own a late therapy has hardly any influence on the number of the metastases. In the case that only the primary tumour is able to spread metastases the quantity of metastases stagnates after the resection. The late therapy has yet no relevant influence in terms of the overall tumour burden (see Figure 4B) [8-10].

The results illustrate the importance of an early diagnosis and the associated early start of the treatment. The continuation of the treatment is of great importance as otherwise the advantage of the early therapy will be lost [10]. 


\section{I nfluence of Natural Killer Cells on the Process of Metastatic Progression}

\subsection{Research question}

The immune system has different defence mechanisms to recognize and eliminate malignant cells. Natural Killer (NK) cells play an important role in the fight against tumour cells as their main function is to eliminate malignant cells [17-19]. If a NK cell is slated for killing, it releases granules, which contain different toxic proteins and consequently destroy malignant cells. Several studies shows that a decreased activity of NK cells facilitates metastatic progression, while with a higher activity slows this process down [20-22].

With the help of our computer model it was determined on which part of the metastatic spread NK cells have the biggest impact. In particular the influence on the growth of the primary tumour, the survival of the tumour cells in the blood and the formation of metastases was examined [8-11].

\subsection{Simulated scenarios}

For this purpose, data from experiments with mouse models were analysed. Rag2 mice do not have the ,recombination-activating gene 2" (rag2) which leads to the dysfunction of B- and T-lymphocytes, hence a specific immune response is absent in these mice. Therefor human tumour cells can be engrafted in this mouse strain. Pfp/rag2 mice are additionally devoid of the perforin gene (pfp), which disables important NK-cells activity. In mice from both strains $10^{6}$ human HT29 colon cancer cells were injected subcutaneously. After termination of the experiment the primary tumour was measured and the number of spontaneously formed lung metastases was determined.

These numbers served as the basis for the following simulation. In both mouse strains a dormancy of the metastasis was included in the simulations and in the group pfp/rag2 additional a late dormancy. Dormancy occurs directly after extravasation of malignant cells and stops cell proliferation for a given time span. Just after the end of the dormancy the tumour cells resume to proliferate as parametrized. Late dormancy occurs when a metastasis reaches a random size between 10 and 100 cells, remains for a certain time in this dormant state and continues growing after that.

\subsection{Results}

Results of the computer simulation show, that NK cells decelerate the growth of the primary tumour. Additional $80 \%$ of the cells in the blood were eliminated, which were otherwise able to form metastases without the activity of the NK cells. NK cells also make it difficult for the disseminated tumour to settle in the lung for nearly 30 days. These cells remain for that time in a dormant state [8-11].

Experimental data of the group pfp/rag2 could not be fitted with the observed results when the dormant state was set directly after the extravasation in the model. Only with late dormancy the calculated dated correlated with the experimentally obtained data. One possible explanation may be that metastases suffer after some cycles of cell division from nutrient deficiency and thus must first induce angiogenic signals to ensure nutrient supply. Further research is necessary at this point to explain the observed late dormancy [8-11].

\section{Discussion}

Computer simulations were shown to be a valuable tool to examine the process of metastasis formation in addition to experimental research and clinical studies. The developed computer model with its building block structure offers the possibility to investigate different scenarios with events and compartments. These include different descriptions of the tumour growth, spreading rates of metastatic tumour cells and the influence of therapies on tumour growth and metastasis formation. The model provides the possibility to analyse clinical or experimental data in more detail and to verify different model assumption quantitative. Predictions about the development of the metastatic progression can be made based on this model. This is particularly valuable to formulate new hypotheses based on data generated by this simulation.

The current version of our computer model includes an idealized process of therapy and does not consider resistance against the treatment methods. Furthermore, the impact of the radiotherapy on the healthy tissue is not considered. Our models of treatment interventions such as chemotherapy and radiation therapy are currently not validated by clinical data [10]. In the current research modelling of treatment interventions will be further developed with a range of new clinical and experimental data. 
Compared to other existing models (e.g. [13] or [23]), our computer model is not only able to include different growth and spread behaviour of primary tumours and metastases easily but also to include a broad range of treatment interventions such as resection of the primary tumour, chemotherapy, external beam radiation, radio embolization and radio immunotherapy as a single treatment or in combination.

\section{References}

[1] Statistisches Bundesamt (2014) Fachserie 12 Reihe 4: Todesursachen in Deutschland 2013. Statistisches Bundesamt, Wiesbaden.

[2] Talmadge JE, Fidler IJ (2010) AACR centennial series: the biology of cancer metastasis: historical perspective. Cancer Res 70:5649-5669. doi: 10.1158/00085472.CAN-10-1040.

[3] Weinberg RA (2006) The Nature of Cancer. In: Biol Cancer. Garland Science, pp 25-56.

[4] Bernhardt P, Ahlman H, Forssell-Aronsson E (2003) Model of metastatic growth valuable for radionuclide therapy. Med Phys 30:3227. doi: 10.1118/1.1628851.

[5] Badri H, Ramakrishnan J, Leder K (2015) Minimizing metastatic risk in radiotherapy fractionation schedules. Phys Med Biol 60:N405. doi: 10.1088/00319155/60/22/N405.

[6] Leder K, Pitter K, LaPlant Q, Hambardzumyan D, Ross BD, Chan TA, Holland EC, Michor F (2014) Mathematical modeling of PDGF-driven glioblastoma reveals optimized radiation dosing schedules. Cell 156:603616. doi: 10.1016/j.cell.2013.12.029.

[7] Benzekry S, Barbolosi D, Benabdallah A, Hubert F, Hahnfeldt P (2013) Quantitative Analysis of the Tumor/Metastasis System and its Optimal Therapeutic Control.

[8] Wedemann G, Bethge A, Haustein V, Schumacher U (2014) Computer simulation of the metastatic progression. In: Metastasis Res Protoc, 2nd ed. Springer, New York, pp 107-116.

[9] Bethge A, Schumacher U, Wree A, Wedemann G (2012) Are metastases from metastases clinical relevant? Computer modelling of cancer spread in a case of hepatocellular carcinoma. PLOS ONE 7:e35689. doi: 10.1371/journal.pone.0035689.
[10] Bethge A, Schumacher U, Wedemann G (2015) Simulation of metastatic progression using a computer model including chemotherapy and radiation therapy. $J \mathrm{Bi}$ omed Inform 57:74-87. doi: 10.1016/j.jbi.2015.07.011.

[11] Brodbeck T, Nehmann N, Bethge A, Wedemann G, Schumacher U (2014) Perforin-dependent direct cytotoxicity in natural killer cells induces considerable knockdown of spontaneous lung metastases and computer modelling-proven tumor cell dormancy in a HT29 human colon cancer xenograft mouse model. Mol Cancer 13:244. doi: 10.1186/1476-4598-13-244.

[12] Tyszer J (1999) Object-oriented computer simulation of discrete-event systems. Kluwer Academic Publ., Boston, Mass.

[13] Iwata K, Kawasaki K, Shigesada N (2000) A dynamical model for the growth and size distribution of multiple metastatic tumors. J Theor Biol 203:177-186. doi: 10.1006/jtbi.2000.1075.

[14] World Wide Web Consortium (2008) Extensible Markup Language (XML) 1.0 (Fifth Edition).

[15] Bethge A, Wedemann G (2014) CaTSiT - Computer simulation of metastatic progression and treatments. [Online]. Available at: http://bioinformatics.fhstralsund.de/catsit/. [Accessed: 12-Nov-2015].

[16] Bethge A (2015) Computersimulation der Metastatischen Progression und Interventionen. Dissertation, Universität Rostock.

[17] Biron CA (1997) Activation and function of natural killer cell responses during viral infections. Curr Opin Immunol 9:24-34.

[18] Trinchieri G (1989) Biology of natural killer cells. Adv Immunol 47:187-376.

[19] Arina A, Murillo O, Dubrot J, Azpilikueta A, Alfaro C, Pérez-Gracia JL, Bendandi M, Palencia B, HervásStubbs S, Melero I (2007) Cellular liaisons of natural killer lymphocytes in immunology and immunotherapy of cancer. Expert Opin Biol Ther 7:599-615. doi: 10.1517/14712598.7.5.599.

[20] Coca S, Perez-Piqueras J, Martinez D, Colmenarejo A, Saez MA, Vallejo C, Martos JA, Moreno M (1997) The prognostic significance of intratumoral natural killer cells in patients with colorectal carcinoma. Cancer 79:2320-2328. 
[21] Liljefors M, Nilsson B, Hjelm Skog A-L, Ragnhammar P, Mellstedt H, Frödin J-E (2003) Natural killer (NK) cell function is a strong prognostic factor in colorectal carcinoma patients treated with the monoclonal antibody 17-1A. Int J Cancer J Int Cancer 105:717-723. doi: 10.1002/ijc.11139

[22] Ménard C, Blay J-Y, Borg C, Michiels S, Ghiringhelli F, Robert C, Nonn C, Chaput N, Taïeb J, Delahaye NF, Flament C, Emile J-F, Le Cesne A, Zitvogel L (2009) Natural killer cell IFN-gamma levels predict long-term survival with imatinib mesylate therapy in gastrointestinal stromal tumor-bearing patients. Cancer Res 69:3563-3569. doi: 10.1158/0008-5472.CAN-08-3807

[23] Benzekry S, Gandolfi A, Hahnfeldt P (2014) Global dormancy of metastases due to systemic inhibition of angiogenesis. PLOS ONE 9:e84249. doi:

10.1371/journal.pone.0084249

\section{Appendix}

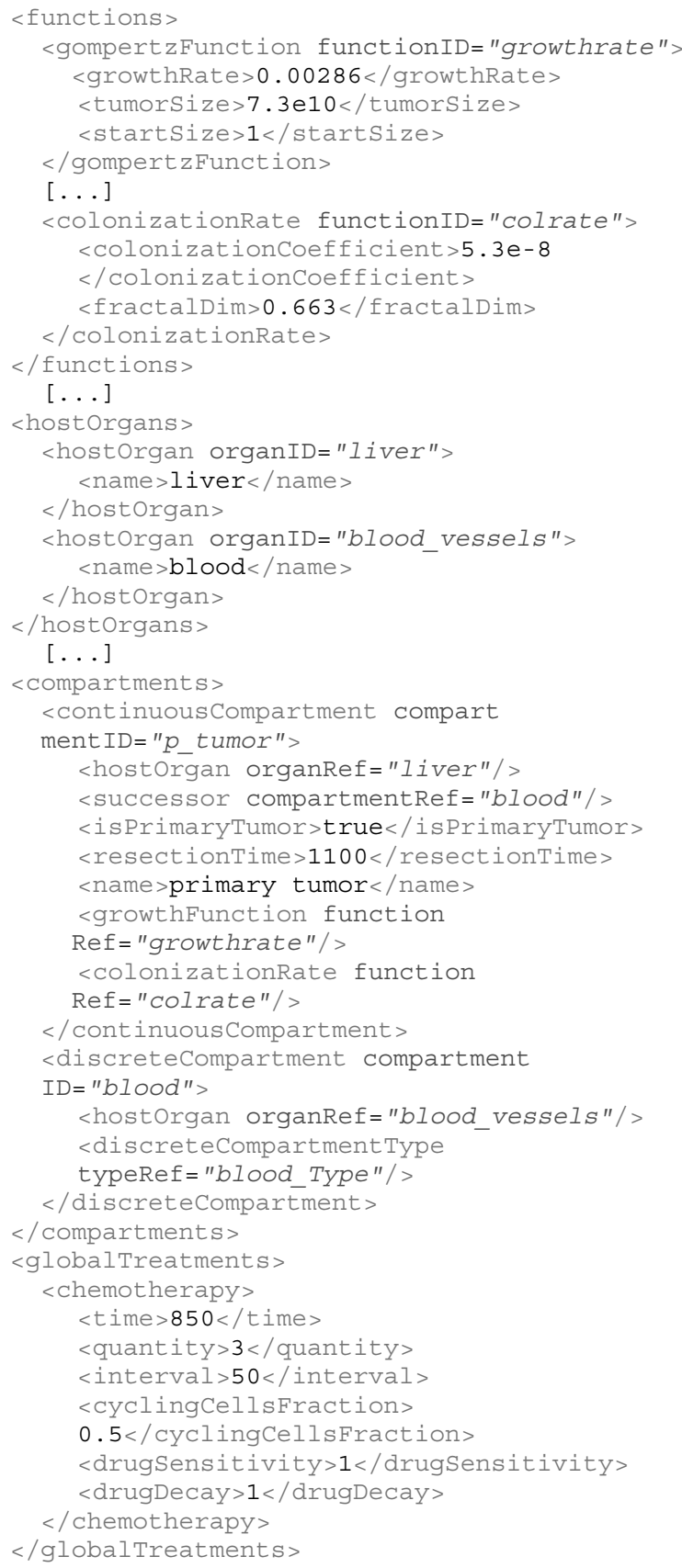

Listing 1. Code snippet of a simulated scenario with a resection of the primary tumour and chemotherapy. In line 2 the growth function and in line 8 the colonization rate is defined. A 3 -cycle chemotherapy is carried out starting on day 850 with an interval of 50 days. The resection of the primary tumour is on day 1100 . 


\section{ASIM - Buchreihen / ASIM Book Series}

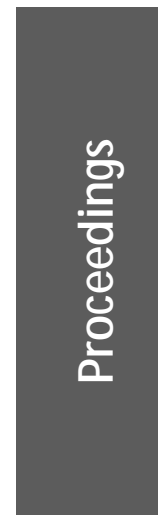

Simulation in Production und Logistics 2015 - 16. ASIM -Fachtagung Simulation in Produktion und Logistik M. Raabe, U. Clausen (Hrsg.); ISBN 978-3-8396-0936-1, Stuttgart: Fraunhofer Verlag, 2015. (O. P.)

Simulation in Produktion und Logistik 2013: Entscheidungsunterstützung von der Planung bis zur Steuerung W. Dangelmaier, C. Laroque, A. Klaas (Hrsg.); ISBN 978-3-942647-35-9, HNI-Verlagsschriftenreihe, Heinz Nixdorf Institut, Paderborn, 2013 (O. P.)

Modellierung, Regelung und Simulation in Automotive und Prozessautomation - Proc. 5. ASIM-Workshop Wismar 2011. C. Deatcu, P. Dünow, T. Pawletta, S. Pawletta (eds.), ISBN 978-3-901608-36-0, ASIM / ARGESIM, Wien, 2011 (O. A.)

Simulation in Produktion und Logistik 2010: Integrationsaspekte der Simulation - Technik, Organisation und Personal. G. Zülch, P. Stock, (Hrsg.), ISBN 978-3-86644-558-1, KIT Scientific Publ. Karlsruhe, 2010 (O.P.)

Simulation und Optimierung in Produktion und Logistik - Praxisorientierter Leitfaden mit Fallbeispielen. L. März, W. Krug, O. Rose, G. Weigert, G. (Hrsg.); ISBN 978-3-642-14535-3, Springer, 2011 (O.P.)

Verifikation und Validierung für die Simulation in Produktion und Logistik - Vorgehensmodelle und Techniken. M. Rabe, S. Spieckermann, S. Wenzel (eds.); ISBN: 978-3-540-35281-5, Springer, Berlin, 2008 (O. P.)

Qualitätskriterien für die Simulation in Produktion und Logistik - Planung und Durchführung von Simulationsstudien. S. Wenzel, M. Weiß, S. Collisi - Böhmer, H. Pitsch, O. Rose (Hrsg.); ISBN: 978-3-540-35281-5, Springer, Berlin, 2008

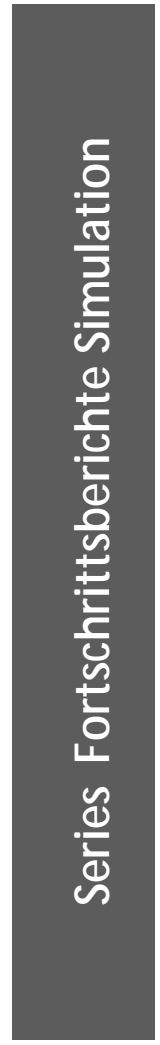

P. Einzinger: A Comparative Analysis of System Dynamics and Agent-Based Modelling for Health Care Reimbursement Systems. FBS 25, ASIM/ARGESIM Vienna, 2014; ISBN 978-3-901608-75-9, ARGESIM Report 75 (0. A.)

M. Bruckner: Agentenbasierte Simulation von Personenströmen mit unterschiedlichen Charakteristiken FBS 24, ASIM / ARGESIM Vienna, 2014; ISBN 978-3-901608-74-2, ARGESIM Report 74 (O. A.)

S. Emrich: Deployment of Mathematical Simulation Models for Space Management FBS 23, ASIM/ARGESIM Vienna, 2013; ISBN 978-3-901608-73-5, ARGESIM Report 73 (0. A.)

G. M aletzki: Rapid Control Prototyping komplexer und flexibler Robotersteuerungen auf Basis des SBC-Ansatzes. FBS 22, ASIM/ARGESIM Vienna, 2014; ISBN 978-3-901608-72-8, ARGESIM Report 72 (0. A.)

$X$. Descovich: Lattice Boltzmann Modeling and Simulation of Incompressible Flows in Distensible Tubes for Applications in Hemodynamics FBS 21, ASIM / ARGESIM Vienna, 2012; ISBN 978-3-901608-71-1, ARGESIM Report 71 (0. A.)

F. M iksch: Mathematical Modeling for New Insights into Epidemics by Herd Immunity and Serotype Shift FBS 20, ASIM/ARGESIM Vienna, 2012; ISBN 978-3-901608-70-4, ARGESIM Report 70 (0. A.)

S. Tauböck: Integration of Agent Based Modelling in DEVS for Utilisation Analysis: The MoreSpace Project at TU Vienna; FBS 19, ASIM/ARGESIM Vienna, 2012; ISBN 978-3-901608-69-8, ARGESIM Report 69 (O. A.)

Ch. Steinbrecher: Ein Beitrag zur prädiktiven Regelung verbrennungsmotorischer Prozesse FBS 18, ASIM / ARGESIM Vienna, 2010; ISBN 978-3-901608-68-1, ARGESIM Report 68 (O. A.)

O. Hagendorf: Simulation-based Parameter and Structure Optimisation of Discrete Event Systems FBS 17, ASIM/ARGESIM Vienna, 2010; ISBN 978-3-901608-67-4, ARGESIM Report 67 (0. A.)

Orders via ASIM (O. A.) or via Publisher (O. P.):

ASIM / ARGESIM Office Germany, Hochschule Wismar, PF 1210, 23952 Wismar, Germany

ASIM/ARGESIM Geschäftsstelle Österreich, c/ o DWH, Neustiftgasse 57, 1040 Vienna, Austria

Download (some books) via ASIM webpage in preparation

Info: www.asim-gi.org, info@asim-gi.org 


\title{
Implementation, Testing, and Evaluation of Center Selection Methods for Supervised Radial Basis Networks to Enhance Breast Cancer Analysis
}

\author{
Manan Nanavati ${ }^{*}$, Boris R. Bracio \\ Anhalt University of Applied Sciences, Koethen, Germany; *manan.nanavati@live.com
}

Simulation Notes Europe SNE 25(3-4), 2015, 133 - 139

DOI: $10.11128 /$ sne.25.tn. 10303

Received: January 158, 2015 (Selected ASIM SST 2014

Postconf. Publ.); Accepted: August 15, 2015;

\begin{abstract}
Breast cancer is a chronic disease which has been classified as a cancer type having one of the highest mortality rates. An early and accurate diagnosis of any chronic disease plays an important role and can be lifesaving. Numerous research articles state that the role of computerized diagnostic tools supporting the decision making in diagnosis of chronic diseases has increased over the past decade. The presented study implements and evaluates three different artificial neural networks in form of supervised radial basis networks (RBN). The performance of the RBN's in regards to different center selection methods using different clustering algorithm are evaluated with the help of the Wisconsin breast cancer dataset (WBCD) by UCl machine learning repository.
\end{abstract}

\section{Introduction}

Cancer is a disease where the neoplasm or the tumor cells show uncontrolled death which leads to formation of a mass or lumps [1], [2]. Today clinicians face the challenge to screen almost 200 different types of cancer [3]. In regards to breast cancer the World Cancer factsheet [4] lists it as the second most diagnosed cancer across the globe along with the second highest mortality rate. The regain of a healthy state of patients in case of chronic illness depends on an early detection as well as on a proper treatment. The most common methods for breast cancer diagnosis are: (1) surgical biopsy and (2) fine needle aspiration cytology (FNAC).
The physician experience and analytical skills are part of the subset for accurate diagnosis of breast cancer. One of the earliest computerized implementation for cancer diagnosis dates back to 1995 using a linear programming approach [5]. During the following decade several machine learning and data mining implementation followed. The positive results of those implementations led to an increase in usage of such computerized tools and underlined the promising nature of computer assisted diagnosis for chronic disease.

Machine learning like artificial neural networks (ANN's) and support vector machine (SVM) have been proven accurate and fast enough for the disease diagnosis. ANN is a soft computing approach, which processes the input data in an adaptive way, i.e. the designed algorithm involves 'learning' from the past information. After learning, the designed ANN can be specifically used for classification of patterns or prediction or forecasting. ANN has become an accurate method for analysis of clinical data for diagnosis purposes in the linear and non-linear domain [6], [7], [8], [9].

In this research study, RBNs were used for the analysis of breast cancer. The fast learning rate and unique design in its own class makes RBN more dominant in some of the applications than conventional multilayer perceptron networks (MLP). This study involves implementation and evaluation of three different RBN's using supervised learning methods in regards to their centers selection methods:

1. Fixed selection of centers at random [10]

2. Selection of centers using the default $k$-means algorithm of MATLAB - proposed method

3. Selection of centers using 'customized' $k$-means algorithm - proposed method 
The main reason behind choosing the supervised learning algorithm is the accuracy obtained in the end stage when compared to that of an unsupervised method. One of the examples representing dominance of supervised RBN over unsupervised RBN was described in [11].

The breast cancer data used for this study is the Wisconsin Breast Cancer Dataset (WBCD) from the University of California Irvin (UCI) Machine Learning Repository [12].

This paper is organized as follows: Section 1 gives the background information on radial basis networks, WBCD data and the previous work using it. Section 2 describes the methods used for this study with subsections explaining all three designs and its implementation. The results obtained are discussed in Section 3 followed by summary of this study in Section 4 .

\section{Background}

\subsection{Wisconsin breast cancer dataset:}

In this research study, the Wisconsin breast cancer dataset available on [12] was used for breast cancer analysis using a RBN. The original data consists of recordings from 699 patients towards their FNAC findings which accumulates 9 different attributes on a scale of $0-10$. In each of the data 10 was classified as most abnormal value and 1 as most normal value, also the class labeled for diagnosis was assigned, ' 2 ' stands for benign and ' 4 ' for malignant breast cancer. According to WBCD original data out of 699 patients, 458 patients were classified into the benign class cancer and 241 as malignant. The FNAC recorded attributes are as follows:

- Clump thickness

- Uniformity of cell size

- Uniformity of cell shape

- Marginal adhesion

- Single epithelial cell size

- Bar nucleoli

- Bland chromatin

- Normal nucleoli

- Mitoses
In the pre-processing part of the data, the original WBCD data available on [12] contains a total of 16 instances having missing attributes value, thus they have been eliminated from the database before implementing of the RBN. The output class of ' 4 ' as malignant breast cancer was changed to ' 1 ', which states a new set of class values i.e. ' 1 ' representing malignant and ' 2 ' representing benign. The same data was also normalized from $0-1$ scale using a minmax method.

\subsection{Radial Basis Networks:}

A radial basis function is a function whose output value depends on its distance from a center ' $c$ '.

The activation function of $\mathrm{RBN}$ is given as:

$$
\varphi=f\|x-c\|
$$

$\varphi=$ activation function

$x=$ input value

$c=$ center of the radial neuron

Radial basis function networks (RBFN) possess a radial symmetric property in regards to their own centers and are a subset network of MLP's. RBFN have a different design and algorithm, it works on analysis of the data during a learning process and applying a 'best fit' approach during a testing phase.

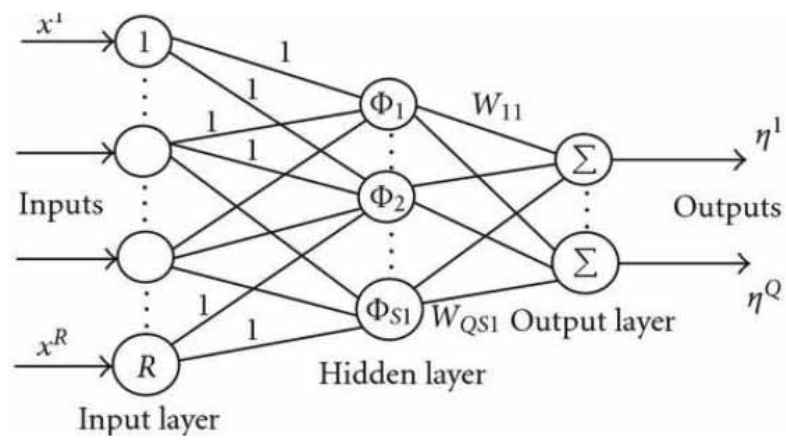

Figure 1: Basic schematic of Radial basis function network [13].

As shown in Figure 1 the construction of a basic RBFN consist of the three different layers:

1. Input Layer: The layer which acts as the source node for the input data.

2. Hidden Layer: It is layer having high number of neurons with a basis function working as the network activation function.

3. Output Layer: It acts as a sink in the network, giving an output response for the given input data. 
Considering all above design constraints, the output response of the radial basis network is given as:

$$
y=\sum w_{i j} \cdot \varphi_{i}(x)
$$

$y=$ output response of network

$\varphi=$ activation function

$x=$ input value

$c=$ center of radial neuron

$w_{i j}=$ weight of output layer

For a Gaussian RBF the activation function is given for a spread value $\sigma$ of the radial neuron as:

$$
\varphi=e^{-\frac{\|x-c\|^{2}}{2 \sigma^{2}}}
$$

The most versatile characteristic of RBFN is that the mapping between input to hidden layer is nonlinear whereas it is linear between the hidden-output layer [10].

\subsection{Previous works on WBCD data:}

The breast cancer diagnosis on WBCD data performed at the University Of Sydney, Australia achieved a 94.74\% accuracy using a C4.5 decision tree algorithm [14]. Researchers at University of Veszprem, Hungary have obtained an overall accuracy of $95.57 \%$ using a fuzzy clustering method [15] whereas researchers at Swiss Federal Institute of Technology, Switzerland have obtained accuracy $97.36 \%$ using a fuzzy genetic algorithm [16]. Researcher at the Tobb University of Economics and Technology, Turkey has demonstrated a SVM machine learning approach which yielded an overall accuracy of $99.54 \%$ [17] where on other side a $100 \%$ accuracy was obtained using a Rough subset theory by SVM at University Changchun, China [18]. When focusing on RBN methods, researcher from the Indian Institute of Management and Technology, India obtained an accuracy of $49.79 \%$ [19], on the other hand researchers at the Yildiz Technical University, Turkey achieved an accuracy of $96.18 \%$ [20].

\section{Methods and Implementation}

As mentioned in Section 1.2, radial basis networks are symmetric to its own center(s), and the output value depends on the distance between input value and the respective center.
Thus, the determination of the center value plays a critical role in the performance of supervised RBN's. In this research study three methods regarding the selection of centers of radial neurons were implemented and their obtained results were compared to each other.

\subsection{Algorithm 1: Fixed selection of centers at random}

In this supervised learning method the random selection of center(s) method described in [10] was used. This was implemented in the form of a radial basis network supervised algorithm present in MATLAB neural network toolbox. The basic steps involved in this method are summarized as follows.

1. Selection of RBN architecture

2. Initialization of network

3. Training of network

4. Validation of the network

5. Test of network

6. Presentation of results

Here the WBCD data for supervised learning were randomly divided for analysis into $70 \%$ training, 15\% validation and $15 \%$ test dataset.

\subsection{Algorithm 2: Selection of centers by default $k$-means algorithm}

The second RBN implementation was also done in MATLAB, but without the neural network toolbox. First a statistical test was used for the determination of a 'Silhouette Index' to calculate the best possible ' $k$ ' value for a k-means clustering on the WBCD data. For this study ' $k=2$ ' was obtained, so two clusters of data will be implemented for this algorithm.

The main aim of using the $k$-means algorithm in this method is to determine the cluster centers for each category input. In this method the default $k$-means algorithm of MATLAB to determine center values is used. It was followed by the design of a supervised RBN for the WBCD analysis. The steps involved in this method are listed as follows: 
1. Determination of Silhouette index

2. Perform $k$-means clustering to determine the centers of input value

3. Calculation of $1 / 2 \sigma^{2}$ term in activation function, where $\sigma$ represents the spread value of radial part

4. Calculate the output activation of radial neurons over inputs

5. Determination of output weights using pseudoinverse method

6. Evaluation of RBN response

7. Presentation of results.

\subsection{Algorithm 3: Selection of centers by modified $k$-means algorithm}

This last RBN was also implemented using MATLAB without using the neural network toolbox. The main aim of using a $k$-means algorithmic approach in this method was to determine the centers for the input categorical data. Furthermore, after a successful determination of a center value using a modified kmeans approach, a supervised RBN was implemented.

The difference between 'Algorithm 2' and 'Algorithm $3^{\prime}$ 'is the method used to calculate the k-means. In Algorithm 2, the given algorithm of MATLAB was used, whereas in 'Algorithm 3' a customized $k$-means approach was designed for the determination of the centers value. The methodological step number 2 to 7 presented in Algorithm 2 in Section 2.2 are same to analyze the WBCD cancer data. The following steps represent the customized k-means algorithm for determination of centers:

1. Determination of number of unique category in target for determining value for $k$-means

2. Selection of Centers per categorial data

3. Selection of intial centroids

4. Perform $k$-means over iterative loop

5. Remove, if any empty clusters present

6. Find the closest centroid to determine membership class.

The performance measure for all three implemented supervised RBN algorithms was the overall accuracy obtained in regards to the total classification rate. The overall accuracy obtained for all three supervised algorithms/methods for centers' determination were compared.

\section{Results and Discussion}

\subsection{Results of Algorithm 1: Fixed selection of centers at random}

The used RBN algorithm in this method for selection of centers is described in [10]. The fixed centers from the categorical input data at random are selected in this method. The algorithm was implemented in MATLAB 7.10 with use of neural network toolbox. The nine different categorical inputs presented in Section 1.1 were taken as the input to the RBN model. The key factor which influences the performance of RBN model is the 'spread' value and centers of the given input data. Here in this method, we have selected centers randomly as mentioned and best spread value was determined using 'spread over loop' method. The following response was obtained when network was simulated with 'loop over spread' method.

The results of implemented model obtained highest overall accuracy rate at spread value of 0.7 represented in Figure 2.

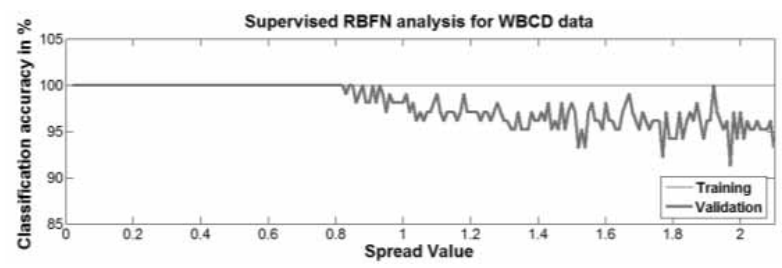

Figure 2: Classification analysis v/s spread values of RBN model.

The following tables, Table 1 and Table 2 represent the confusion matrix obtained through Algorithm 1during training and testing phase.

Training Dataset

\begin{tabular}{lcc}
\hline & Actual Class & \multicolumn{1}{l}{ Predicted Class } \\
\hline & $\begin{array}{l}\text { Positive (M alig- } \\
\text { nant) = 195 }\end{array}$ & $\begin{array}{l}\text { Negative (Benign) } \\
\mathbf{2 2 8 5}\end{array}$ \\
\hline $\begin{array}{l}\text { Positive (Malig- } \\
\text { nant) = 195 }\end{array}$ & 195 & 0 \\
\hline $\begin{array}{l}\text { Negative (Benign) } \\
=\mathbf{2 8 5}\end{array}$ & 0 & 285 \\
\hline
\end{tabular}

Table 1: Results of Training phase- Algorithm 1. 
Test Dataset

\begin{tabular}{lcc}
\hline & Actual Class & \multicolumn{2}{l}{ Predicted Class } \\
\hline & $\begin{array}{l}\text { Positive (Malig- } \\
\text { nant) = 195 }\end{array}$ & $\begin{array}{l}\text { Negative (Benign) } \\
\mathbf{2 2 8 5}\end{array}$ \\
\hline $\begin{array}{l}\text { Positive (Malig- } \\
\text { nant) = 21 }\end{array}$ & 20 & 1 \\
\hline $\begin{array}{l}\text { Negative (Benign) } \\
=79\end{array}$ & 1 & 78 \\
\hline
\end{tabular}

Table 2: Results of Testing phase- Algorithm 1.

The total accuracy for the analysis of results obtained is calculated as:

$$
T . A=\frac{T P+T N}{T P+F P+F N+T N} * 100 \%
$$

$T A=$ Total accuracy

$T P=$ True Positive value

$T N=$ True Negative value

$F P=$ False Positive value

$F N=$ False Negative value

The overall accuracy of $100 \%$ for the training and validation and $98 \%$ for the testing data set was obtained.

\subsection{Results of Algorithm 2: Selection of centers using default $k$-means algorithm of MATLAB}

In this second method, the RBN based diagnostic tool for breast cancer was also implemented and tested on MATLAB 7.10, but here the neural network toolbox was not used. The same set of input parameters as taken in Algorithm 1 were taken as end point and to compare and analyze these algorithms. But here no partition of data was done prior before giving input (i.e. 100\% training dataset). Prior to the design and implementation of supervised RBN model for breast cancer two steps were done: (1) Determination of Silhouette index (The silhouette value for input data is a measure of similarity of points within in its own cluster, compared to the other points in the cluster [21].) (2) k-means clustering for determination of centers. The maximum silhouette index of ' 0.75 ' was obtained at ' $k=2$ '. Later on the obtained ' $k$ ' value was adapted for default k-means algorithm. Then in the same way an implementation of the RBN model and activation function was determined based on the input values.
Finally the learning weights were determined using pseudoinverse method. The result of the same method were implemented and analyzed by calculation of the total accuracy obtained as mentioned in above method. The following table represents the results obtained through implementation of 'Algorithm 2'.

The overall accuracy of 'Algorithm 2' was found to be $96.77 \%$. Here in this method, 2 centers per categorical data were used as the outcome of the default $k$ means algorithm.

\section{Training Dataset}

\begin{tabular}{lc}
\hline & $\begin{array}{c}\text { Number of predicted value } \\
\text { (Benign }+ \text { M alignant) }\end{array}$ \\
\hline Right predicted values & 661 \\
\hline Wrong predicted values & 22 \\
\hline
\end{tabular}

Table 3: Results of Algorithm 2.

\subsection{Algorithm 3: Selection of centers using 'centralized' k-means algorithm}

In this last method, the RBN model for breast cancer diagnosis was also implemented on MATLAB 7.10 but here also no neural network toolbox is used. The same input dataset used in 'Algorithm 1' and 'Algorithm 2' is also used in this method, but likewise no partition of data as mentioned in Section 4.2 is used i.e. $100 \%$ training dataset. Prior before implementation of RBN model for diagnosis, two steps were done: (1) Determination of number of unique categories in target data (2) Perform 'customized' $k$-means for determination of centers. The only difference between both k-means approach is the involved substeps of it.

Here in this method, there is feature added to decide the 'number of centers per category'. Also, empty clusters were also removed, which may lead to higher accuracy, was also implemented in this method. In this method, the total accuracy was recorded over different values of 'number of centers per category'. The other steps like determination of activation function and determination of output weights were same as used in 'Algorithm 2'. The following Table 4 describes the accuracy analysis over different values of "number of category'. Here in this method numbers of centers ranges from 1 to 10 were evaluated for accuracy analysis. 
At the end of all implementation involved in this research study following few things can be summarized when all results are compared in regards to their overall accuracy. All the methods implemented in this study have acheived noticeable performance of $90 \%$ and higher accuracy in the end results obtained. One of our proposed method i.e. Algorithm 3 i.e. 'Selection of centers using customized k-means algorithm' has achieved accuracy of $97.07 \%$ at 3 selected centers per category. On other side no significant difference in overall accuracy was found in higher number of centers per category. Likewise on other side, by use of sophisticated neural network toolbox gave almost same level of accuracy.

\begin{tabular}{cccc}
\hline $\begin{array}{l}\text { Number of } \\
\text { centers per } \\
\text { category }\end{array}$ & $\begin{array}{l}\text { Total overall } \\
\text { accuracy }\end{array}$ & $\begin{array}{l}\text { Number of } \\
\text { centers per } \\
\text { category }\end{array}$ & $\begin{array}{l}\text { Total overall } \\
\text { accuracy }\end{array}$ \\
\hline $\mathbf{1}$ & $96.48 \%$ & 6 & $96.92 \%$ \\
\hline $\mathbf{2}$ & $96.92 \%$ & 7 & $96.92 \%$ \\
\hline $\mathbf{3}$ & $\mathbf{9 7 . 1 0} \%$ & 8 & $96.92 \%$ \\
\hline $\mathbf{4}$ & $96.77 \%$ & 9 & $96.92 \%$ \\
\hline $\mathbf{5}$ & $96.92 \%$ & 10 & $96.92 \%$ \\
\hline
\end{tabular}

Table 4: Accuracy outcome obtained in Algorithm 3.

\begin{tabular}{lc}
\hline & $\begin{array}{l}\text { Number of predicted value } \\
\text { (Benign + Malignant) }\end{array}$ \\
\hline Right predicted values & 663 \\
\hline Wrong predicted values & 20 \\
\hline
\end{tabular}

Table 5: Results for number of selected centers $=3$ (Algorithm 3).

\section{Conclusions}

The main purpose of this research work was to compare performance of several centers selection methods for developing RBN models. The overall classification rate obtained at the end of this study stated that, all the implemented supervised RBN models show higher accuracy rate of $90 \%$ and above.
The noticeable performance of $97.07 \%$ was obtained in last method based on 'number of centers per category' choice. On the other side the other two methods i.e. basic supervised RBN algorithm i.e. method 1 showed total accuracy of $98 \%$ and method 2 with basic k-means showed total accuracy of $96.77 \%$. When comparing all above results obtained, significant differences were found. This states that there is dominance of centers selection for performance of RBN model. The noticeable result obtained in this study also states: the algorithm without neural network toolbox showed almost the same results as that of the algorithm involving sophisticated toolbox.

Thus, in the end to summarize the research work we conclude that there is a critical role of centers determination in performance of RBN when accuracies of all methods are compared to each other, as it is clear from the definition that RBN are radially symmetric in regards to their own centers. In future sophisticated algorithms to determine centers can be developed for evaluation of RBN which further can be compared to standard supervised RBN algorithm of MATLAB which may produce noticeable results.

\section{References}

[1] American Cancer Society. Cancer Glossary. cancer.org. Retrieved September 11, 2013.

[2] National Cancer Institute. What is cancer?. cancer.gov. Retrieved September 11, 2013.

[3] Cancer Research UK: CancerHelp UK. How many different types of cancer are there?. Retrieved: 11 May 2012.

[4] International Agency for Research on Cancer and Cancer Research UK. World Cancer Factsheet. Cancer Research UK, London, 2014.

[5] Mangasarian OL, Wolberg WH. Cancer diagnosis via linear programming. SIAM News. 1990; vol. 23(5): 1 18.

[6] Cichocki A, Unbehauen R. Neural Networks for optimisation and signal processing. J. Wiley, Sons Ltd. And B.G.Teubner, Stuttgart: 1993.

[7] Ahmed A, Medhat M, Muhamed FW. Using data mining for assessing diagnosis of breast cancer. Proc. International multiconference on computer science and information Technology, 2010, 11-17.

[8] Burke HB et al. Artificial Neural Networks Improve the Accuracy of Cancer Survival Prediction. Cancer. 1997, vol.79, pp.857-862. 
[9] Revett K, Gorunescu F, Gorunescu M, El-Darzi E, Marius. A Breast Cancer Diagnosis System: A CombinedApproach Using Rough Sets and Probabilistic Neural Networks. EUROCON 2005, 2005 Nov, Serbia \& Montenegro, Belgrade, 22- 24, 1124-1127.

[10] Haykin, S. Neural networks: acomprehensive foundation. New York:Macmillan. 1994.

[11] Wilamowski BM, Vieira K. Clustering of patterns using radial base function networks. In Artificial Neural Networks in Engineering ANNIE'95. 1995; 109-115.

[12] UCI Repository of Machine Learning Databases. www.archive.ics.uci.edu/ml $/$ machinelearningdatabases/breast-cancer-wisconsin/

[13] Ab Malek MN, Mohamed Ali MS. Evolutionary Tuning Method for PID Controller Parameters of a Cruise Control System Using Metamodeling. Modelling and Simulation in Engineering. 2009; vol. 2009: 8 pages. doi:10.1155/2009/234529.

[14] Quinlan JR. Improved use of continuous attributes in C4.5. J. Artif. Intell. Res. 1996; 4: pp. 77-90.

[15] Abonyi J, Szeifert F. Supervised fuzzy clustering for the identification of fuzzy classifiers. Pattern Recognition Lett. 2003; 24: 2195-2207.
[16] Pena-Reyes CA, Sipper M. A fuzzy-genetic approach to breast cancer diagnosis. Artif. Intell. Med. 1999;17: 131155 .

[17] Übeyli, ED. Implementing automated diagnostic systems for breast cancer detection. Expert Systems with Applications. 2007; 33(4): 1054-1062.

[18] Chen HL, Yang B, Liu J, Liu DY. A support vector machine classifier with rough set-based feature selection for breast cancer diagnosis. Expert Systems with Applications. 2011; 38(7): 9014-9022.

[19] Janghel, R. R., Anupam Shukla, Ritu Tiwari, and Rahul Kala. Breast cancer diagnosis using artificial neural network models. In 3rd International Conference on Information Sciences and Interaction Sciences (ICIS), 2010; 89-94. IEEE.

[20] Kiyan, Tüba, and Tülay Yildirim. Breast cancer diagnosis using statistical neural networks. IUJournal of Electrical \& Electronics Engineering. 2011; 4(2): 11491153.

[21] Kaufman L, P. J. Rousseeuw PJ. Finding Groups in Data: An Introduction to Cluster Analysis. Hoboken, NJ: John Wiley \& Sons, Inc.; 1990. 


\section{EUROSIM 2016 $9^{\text {th }}$ EUROSIM Congress on Modelling and Simulation}

City of Oulu, Finland, September 12 - 16, 2016
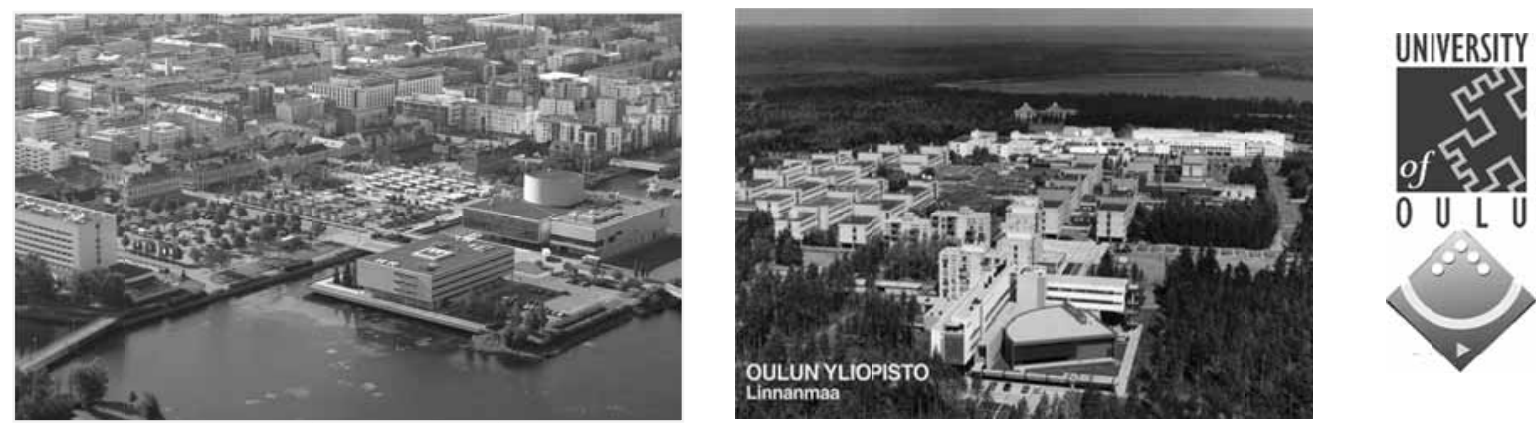

EUROSIM Congresses are the most important modelling and simulation events in Europe. For EUROSIM 2016, we are soliciting original submissions describing novel research and developments in the following (and related) areas of interest: Continuous, discrete (event) and hybrid modelling, simulation, identification and optimization approaches. Two basic contribution motivations are expected: M\&S Methods and Technologies and M\&S Applications. Contributions from both technical and non-technical areas are welcome.

Congress Topics The EUROSIM 2016 Congress will include invited talks, parallel, special and poster sessions, exhibition and versatile technical and social tours. The Congress topics of interest include, but are not limited to:

Intelligent Systems and Applications Hybrid and Soft Computing Data \& Semantic Mining Neural Networks, Fuzzy Systems \& Evolutionary Computation Image, Speech \& Signal Processing Systems Intelligence and Intelligence Systems Autonomous Systems Energy and Power Systems Mining and Metal Industry Forest Industry

Buildings and Construction Communication Systems

Circuits, Sensors and Devices

Security Modelling and Simulation
Bioinformatics, Medicine, Pharmacy and Bioengineering

Water and Wastewater Treatment, Sludge Management and Biogas Production

Condition monitoring, Mechatronics and maintenance

Automotive applications

e-Science and e-Systems

Industry, Business, Management, Human Factors and Social Issues

Virtual Reality, Visualization,

Computer Art and Games

Internet Modelling, Semantic Web and Ontologies

Computational Finance \& Economics
Simulation Methodologies and Tools Parallel and Distributed Architectures and Systems Operations Research Discrete Event Systems Manufacturing and Workflows Adaptive Dynamic Programming and Reinforcement Learning Mobile/Ad hoc wireless networks, mobicast, sensor placement, target tracking Control of Intelligent Systems Robotics, Cybernetics, Control Engineering, \& Manufacturing

Transport, Logistics, Harbour, Shipping and Marine Simulation

Congress Venue / Social Events The Congress will be held in the City of Oulu, Capital of Northern Scandinavia. The main venue and the exhibition site is the Oulu City Theatre in the city centre. Pre and Post Congress Tours include Arctic Circle, Santa Claus visits and hiking on the unique routes in Oulanka National Park.

Congress Team: The Congress is organised by SIMS - Scandinavian Simulation Society, FinSim - Finnish Simulation Forum, Finnish Society of Automation, and University of Oulu. Esko Juuso EUROSIM President, Erik Dahlquist SIMS President, Kauko Leiviskä EUROSIM 2016 Chair

Info: eurosim2016.automaatioseura.fi, office@automaatioseura.fi 


\title{
Evaluation of Risk Factors for Parametrization of Cancer Models
}

\author{
Andreas Bauer ${ }^{1 *}$, Günther Zauner ${ }^{2}$, Christoph Urach ${ }^{2}$ \\ ${ }^{1}$ Department of Analysis and Scientific Computing, Vienna University of Technology, Wiedner Haupstraße 8-10, \\ 1040 Vienna, Austria; *andreas.e101.bauer@tuwien.ac.at \\ ${ }^{2}$ dwh Simulation Services, Neustiftgasse 57-59, Vienna, Austria
}

Simulation Notes Europe SNE 25(3-4), 2015, 141 - 144

DOI: $10.11128 /$ sne.25.sn.10304

Received: January 18, 2015 (Selected ASIM SST 2014

Postconf. Publ.); Accepted: July 21, 2015;

Abstract. Cancer is the second most cause of death in Austria and around 38000 people are diagnosed with cancer each year [1]. The goal of this paper is to analyze methods for evaluation of risk factors in order to parametrize a micro simulation model for cancer prevalence. The focus of this paper is on modeling the survival time. This is done by the methods of survival analysis and model selection. Firstly, the survival function is estimated by the Kaplan-Meier estimate. Afterwards, a Cox proportional hazards regression is performed with all possible sets of parameters. These models are tested by twos with the likelihood ratio test in order to compare them.

Another approach is the so-called Lasso method. This method puts a constraint on the sum of the absolute values of the regression coefficients and in most cases forces some of the coefficients to go to zero. The Akaike Information Criterion is also applied. All three methods are compared and the parameters which are supported, at least to a certain extent, by all of them are included in the estimation of the survival time of the prevalence model.

\section{Introduction}

Cancer is the second most cause of death in Austria and around 38000 people are diagnosed with cancer each year [1].

So, it is of great importance to find out the risk factors on one side, but also to model the incidence and prevalence to be able to evaluate health policy measures on the other side.
An important step for doing a simulation is to find out the potential influences on the course of the disease and to quantify them in order to parametrize the model.

The goal of this paper is to test methods for identification of possible influence factors on the course of cancer and to do a survival analysis for finding out the factors on which the course of the disease depends. Also, methods of model selection are used. These analyses will be used for the parametrization of a microsimulation model for cancer prevalence later on.

\section{Data}

The following six categories are examined to find out, if they are possible influences on the development or the course of cancer: sex, age at the diagnosis date, chronic diseases $\mathrm{X}, \mathrm{Y}$ and $\mathrm{Z}$ and the stage of cancer at the date of the diagnosis. In Table 1, an overview of these categories with according types and ranges is presented.

\begin{tabular}{l|l|l|l} 
Number & Category & Type & Range \\
\hline 1 & Age & ratio & $23-83$ \\
\hline 2 & Sex & nominal & 0,1 \\
\hline 3 & Chronic disease Y & ordinal & 0,1 \\
\hline 4 & Chronic disease Z & ordinal & 0,1 \\
\hline 5 & Chronic disease X & ordinal & 0,1 \\
\hline 6 & Stage of disease & ordinal & $2-4$
\end{tabular}

Table 1: Overview of categories with according types and ranges. 


\section{Methods}

\subsection{Survival Analysis}

In order to examine the survival time of the individuals depending on the possible influence factors, methods of survival analysis are applied. These methods allow the estimation and the analysis of the survival function and the hazard function. The survival function $S(t)$ is defined as the probability that an individual will survive up to time $t$ and the hazard function $h(t)$ is defined as the instanteneous rate of death at time $t$. The cumulative hazard function $\Lambda$ is given by $\Lambda(t)=\int_{0}^{t} h(x) d x$.

Another important aspect regarding survival analysis is censoring. In the field of survival analysis often the data collection ends before the event of interest has occurred for all individuals. For those individuals, the survival time cannot be determined. The only thing that is known is that the survival time exceeds the time of the observation of the particular patient.

Kaplan-Meier Estimate. The Kaplan-Meier estimate is an estimate for the survival function $S(t)$. It makes use of the information of the exact date of the occurrence of death. The estimated survival ity $s_{t}$ at time $t$ is:

$$
s_{t}=\frac{n_{t}-d_{t}}{n_{t}}
$$

$n_{t}$ is the number of people alive at time $t$ and $d_{t}$ is the number of people that died at time $t$. So, $s_{t}$ is simply the ratio of the people alive who survive time $t$. Thus, the probability of surviving up to a certain point of time $t_{j}$ is calculated with the so-called product-limit formula [2]:

$$
S\left(t_{j}\right)=\prod_{i=1}^{j} S\left(t_{i}\right)
$$

Nelson-Aalen Estimate. The Nelson-Aalen estimate is an estimate for the cumulative hazard function. Let $d_{-}$tand $n_{-} t$ denote the numbers of people that experience the event at time $t$ respectively are at risk at time t. Let $t \_i$ denote the event times. Then, $\Lambda$ can be estimated by

$$
\widehat{\Lambda}(t)=\sum_{i: t_{i} \leq t} \frac{d_{t_{i}}}{n_{t_{i}}}
$$

Cox Regression. A common approach to do regression analysis on survival data is the so-called Cox regression, also known as proportional hazards regression. It assumes that the ratio of the hazards comparing different exposure groups remains constant over time. This is called the proportional hazards assumption. The mathematical form of the proportional hazards model is:

$$
h(t)=h_{0}(t) * \exp \left(\sum_{i=1}^{n} b_{i} * x_{i}\right)
$$

$h_{0}(t)$ denotes the baseline hazard which refers to a particular group of individuals (for example, the individuals with value zero in all binary categories, with mean age and with stage of illness two), $n$ is the number of covariates, $x_{i}$ is the value of the $i$ th covariate and $b_{i}$ is the corresponding regression coefficients $[3,4]$.

\subsection{Model Selection}

The methods of model selection can be used to find the significant covariates for our model depending on given data. The goal of model selection is to eliminate some of the covariates from the full model with six covariates to get a simpler model which still explains most of the effects correctly. In order to find an appropriate model, three approaches are considered: Likelihood ratio tests, Lasso - Method and Akaike Information Criterion (AIC).

Firstly, the Cox regression is performed with all possible sets of parameters. That means the parameter sets of the models are all possible subsets of the full set with six parameters.

Likelihood Ratio Tests. For each two nested models the likelihood ratio test is applied. With this test we examine, if the bigger model of the two significantly provides additional information in comparison to the smaller nested model. The significance level is set to 0.05 .

Lasso-Method. Another approach to select a model is to use the Lasso-method. The regression coefficients of the Cox regression are calculated as usual by minimizing the partial log-likelihood, but additionally the sum of the absolute values of the regression coefficients is bounded by a copjant in order to force some of the coefficient to shrink to zero. This results in a sequence of models depending on the size of the constraint. 
There are various ways to determine the 'best' size of the constraint. It can be either chosen arbitrarily or automatically based on the data. For instance, the use of an approximate generalized cross-validation (GCV) statistic is a common tool for automatic constraint selection [5].

Akaike Information Criterion. Another criterion to select a set of variables is the so-called "Akaike Information Criterion" (AIC). The AIC value is calculated as follows:

$$
A I C=-2 \log \mathcal{L}\left(t_{0} \mid y\right)+2 K
$$

The first summand is the negative of twice the numerical value of the log-likelihood at its maximum point $t_{0}$ given data $y$ and the second summand is twice the number of parameters of the model. The smaller the AIC value of a model is, the better it is, because the AIC value can be interpreted as a kind of information loss [6]. The AIC can also be used for automatic choice of a constraint for the abovementioned Lasso-method.

\section{Results}

The Kaplan-Meier estimate was calculated for various groups of the population. Figure 1 shows the KaplanMeier estimates for male and female individuals in comparison. We can see that the estimate for the males is lower than the estimate for the females until about 800 days after the diagnosis, when only 10 percent of the individuals are left alive.

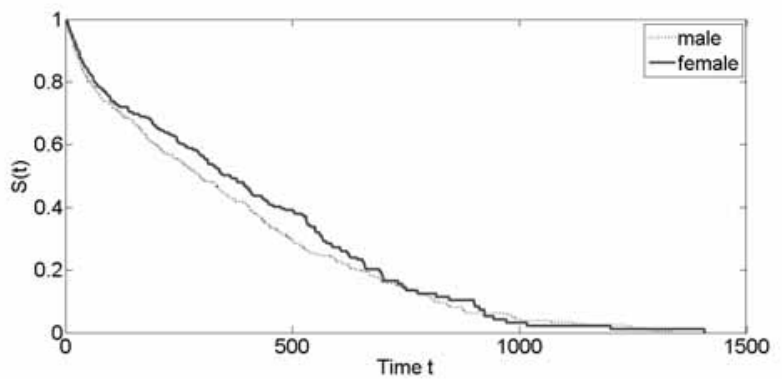

Figure 1: Comparison of Kaplan-Meier estimates for male and female individuals

The coefficients for the Cox regression with all six covariates included are shown in Table 2.

\begin{tabular}{|c|c|c|c|c|c|}
\hline $\boldsymbol{b}_{\mathbf{1}}$ & $\boldsymbol{b}_{\mathbf{2}}$ & $\boldsymbol{b}_{\mathbf{3}}$ & $\boldsymbol{b}_{\mathbf{4}}$ & $\boldsymbol{b}_{\mathbf{5}}$ & $\boldsymbol{b}_{\mathbf{6}}$ \\
\hline-0.11 & 0.02 & -0.05 & 0.09 & -0.25 & 0.02 \\
\hline
\end{tabular}

Table 2: Coefficients of Cox regression in order: sex, age, chronic disease $Y$, chronic disease $Z$, chronic disease $X$, stage of illness.

The p-values of the Cox regression show that the pvalue of the term age is the lowest, so we start with the model with only term age. The likelihood ratio test shows that the model with added terms chronic disease $\mathrm{X}$ and sex and the model with added term chronic disease $\mathrm{X}$, both given the term age, are statistically significant, while any other extension given the term age is not significant.

Figure 2 shows the values of the six regression coefficients of the Cox model plotted over the $l_{1}$ - norm of the coefficient vector. On the $\mathrm{x}$-axis above the plot also the number of non-zero coefficients is displayed.

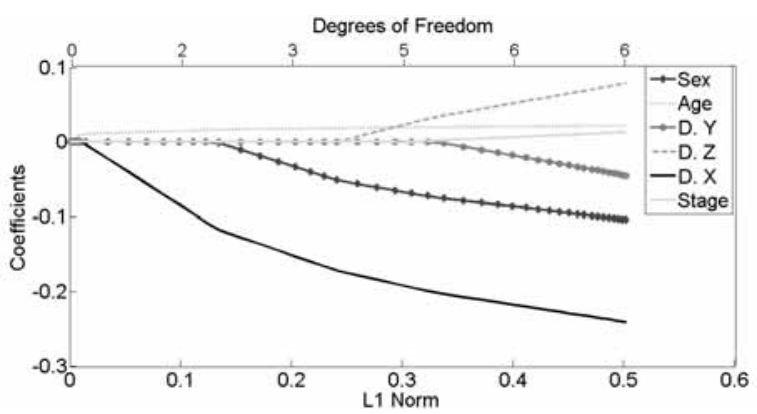

Figure 2: Cox regression coefficients over the norm of coefficients vector.

In Figure 2, we see that the smaller the norm of the vector gets, the smaller is the number of non-zero coefficients. The coefficient that vanishes at last, when the norm of the coefficient vector goes to zero, is the coefficient of the parameter age, right after the coefficients of the parameters chronic disease $\mathrm{X}$ and sex. The other three coefficients are eliminated earlier.

The AIC value is calculated for the models with all 64 possible sets of variables. In order to compare different models, AIC differences are computed, because the relative values of the AIC are more meaningful than the absolute values. The AIC differences are computed by subtracting the AIC value of the model with the least AIC value from the AIC values of each model. 
In Table 3, the five models with the least AIC values and the AIC differences are listed.

\begin{tabular}{|l|l|l|}
\hline Parameters & AIC & AIC difference \\
\hline $1 ; 2 ; 5$ & 6788.75 & 0 \\
\hline $2 ; 5$ & 6789.15 & 0.40 \\
\hline $1 ; 2 ; 3 ; 5$ & 6790.27 & 1.52 \\
\hline $1 ; 2 ; 4 ; 5$ & 6790.41 & 1.66 \\
\hline $1 ; 2 ; 5 ; 6$ & 6790.59 & 1.84 \\
\hline
\end{tabular}

Table 3: Parameters sets with lowest AIC values and AIC differences, numbers referred to numbering of categories in Table 1.

Table 3 shows that the model with parameters sex, age and chronic disease $\mathrm{X}$ has the lowest AIC-value followed by the model with parameters age and chronic disease $\mathrm{X}$. The other three listed models are also substantially supported by the AIC.

\section{Conclusion and Outlook}

The categories age, sex and chronic disease $\mathrm{X}$ are found to be significant by likelihood ratio tests using the Cox regression. The AIC ranks this model as first too. The Lasso-Method shows that these three parameters are the last three parameters that are left, when the norm of the coefficient vector declines. Since the set of parameters is not very big, in uncertain situations, where it is not obvious, if a certain parameter should be included or not, the parameter will be included in the model to avoid the situation that a substantial effect is eliminated from the model by accident.
So all used methods suggest that these three parameters definitely should be included in the estimation of the survival time for the prevalence model. For the other categories, further analysis will be done to determine, if they also will be included in the future model.

\section{Acknowledgement}

This paper has already been published in the proceedings of the " 22 nd $A S I M 2014$ Symposium Simulationstechnik".

\section{References}

[1] Jahrbuch der Gesundheitsstatistik. Wien: Statistik Austria; 2012.

[2] Kirkwood BR, Sterne JAC. Essential Medical Statistics, $2^{\text {nd }}$ edition. Oxford: Wiley-Blackwell; 2003. 501p.

[3] Rodriguez G. Lecture Notes on Generalized Linear Models. Princeton: Princeton University; 2007.

[4] Cox DR, Oakes D. Analysis of Survival Data. Chapman \& Hall, United Kingdom, 1984.

[5] Tibshirani R. The Lasso Method for Variable Selection in the Cox Model. Statistics in Medicine. 1997; Volume 16. p. 385-395.

[6] Burnham KP, Anderson DR. Model Selection and Multimodel Inference: A Practical Information-Theoretic Approac., $2^{\text {nd }}$ edition. New York: Springer; 2002. 


\title{
Nonlinear Methods in Heart Rate Variability: Can they Distinguish between Nonpathological and Pathological Subjects?
}

\author{
Stefan Hagmair ${ }^{1 *}$, Martin Bachler ${ }^{1,2}$, Siegfried Wassertheurer ${ }^{1}$, Christopher Mayer $^{1}$ \\ ${ }^{1}$ AlT Austrian Institute of Technology GmbH, Health \& Environment Department, Biomedical Systems, \\ Donau-City- Straße 1,1220 Vienna, Austria; ${ }^{*}$ stefan.hagmair.fl@ait.ac.at \\ ${ }^{2}$ Department of Analysis and Scientific Computing, Vienna University of Technology, Wiedner Haupstraße 8-10, \\ 1040 Vienna, Austria
}

Simulation Notes Europe SNE 25(3-4), 2015, 145-150

DOI: $10.11128 /$ sne.25.tn. 10305

Received: February 15, 2015; Revised September 20, 2015; Accepted: October 10, 2015

Abstract. Heart Rate Variability (HRV), i. e., the variation of time intervals between consecutive heart beats, is a marker of the health status, since it unveils changes in beat-to-beat variation of the heart, even before there is a remarkable change in heart rate itself. HRV reflects the balance between the sympathetic and the parasympathetic nervous system. The heart rate itself is nonstationary and the structure generating the signal involves nonlinear contributions. Thus, nonlinear methods to quantify the variability of the heart rate gained interest over the last years. In this work, two nonlinear indices, i.e., Correlation Dimension (CD) and Fractal Dimension (FD), to quantify HRV derived from mathematical models are presented. The implemented methods are tested on their ability to differentiate between healthy and pathological subjects. The databases used for the test are retrieved from PhysioNet. The results show that the FD is able to differentiate between nonpathological and pathological subjects, while the other implemented method, i. e., CD, shows no significant difference. In summary, this paper shows that fractal descriptors are an appropriate support for analyzing the $H R V$, and therefore help to prevent or detect cardiovascular diseases. Especially Higuchi's Fractal Dimension, well established in the analysis of time series, should get more attention in analyzing biomedical signals, such as HRV.

\section{Introduction}

An epidemiological update for the Cardiovascular Diseases (CVD) Statistics in Europe 2015 reports that cardiovascular diseases are the most common cause of mortality in Europe and globally. Furthermore, CVD causes almost two times as many deaths as cancer across Europe [1].

Several papers showed the significant relationship between Autonomic Nervous System (ANS) and cardiovascular mortality [2]. Heart Rate Variability (HRV) analysis is a useful noninvasive tool for understanding and characterizing the status of the ANS. Furthermore, HRV is an early predictor of cardiac failures [3]. The term HRV refers to the variation of beat-to-beat intervals. Usually, HRV is studied in an Electrocardiogram (ECG) by considering the time duration between two R-peaks. Therefore, the beat-to-beat intervals are often named RR-intervals. A schematic representation of an ECG-signal can be seen in Figure 1.

The complex origin of biomedical signal limits the traditional linear approaches [4]. Hence, nonlinear methods gained recent interest to reveal more information embedded in the Heart Rate [5]. In this article two nonlinear indices are presented and compared.

\section{Data and Test}

\subsection{Data}

All data used for the tests have been taken from PhysioNet [6], a free-access, online archive of physiological signals. PhysioNet guarantees that all data have been fully deidentified (anonymized), and may be used without further institutional review board approval. 


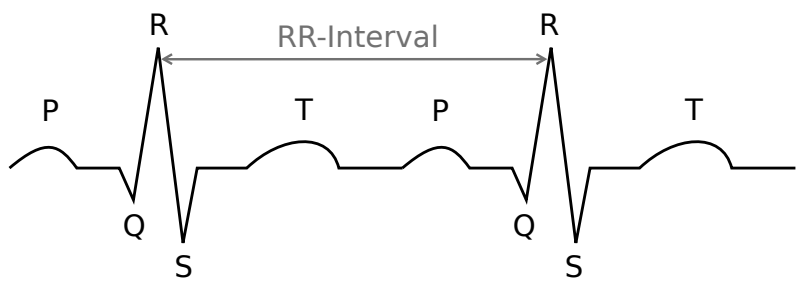

Figure 1: Illustration of an ECG-signal. The beat-to-beat interval (i. e., RR-interval) is defined between two consecutive R-peaks.

The Normal Sinus Rhythm RR-Interval Database [6] defines the control group. It is a composition of 54 approximately 24 hours long ECG recordings. The 30 healthy men are 28.5 to 76 years old and the 24 woman range from 58-73 years. Sample frequency of the signals is $128 \mathrm{~Hz}$.

As pathological dataset, the MIT-BIH Arrhythmia Database [7] was used. It is a collection of 48 halfhour recordings from 47 subjects ( 25 men aged 32 to 89 years and 22 woman aged 23 to 89 years). 25 examples of uncommon but clinically important arrhythmias are included in this database and the remaining recordings include different pathologies. The digitization rate is $360 \mathrm{~Hz}$.

Before calculation of HRV indices the data were processed. The first step of preprocessing was to remove all intervals longer than two seconds. This threshold for the deletion of very long intervals is reported in [8]. Afterwards data was clustered in order to perform the calculations only on the sinus beat cluster. The clustering method is described in detail in $[9,10]$. The required parameters were set to a number of iterations $r=20$ and the minimum number of points in one cluster MinPts $=4$.

From all of the recordings, the middle 1500 RRintervals were taken. After processing the data, measures were calculated on the first 1024 RR-intervals. The last four recordings, i. e., 231-234 were excluded from the pathological database, since after clustering less than $1024 \mathrm{RR}$-intervals were available.

\begin{tabular}{lcc}
\hline & Nonpathological & Pathological \\
\hline records (female) & $54(24)$ & $48(22)$ \\
$\mathbf{N}_{\text {min }}$ & 77373 & 1512 \\
\hline
\end{tabular}

Table 1: PhysioNet databases. $N_{\min }$ is the minimal number of RR-intervals per database.

\subsection{Test}

To test if the models can differentiate between healthy and unhealthy subjects, indices of the pathological database were tested against the calculated indices of the nonpathological database. Since the results follow a normal distribution, the t-test was used to determine the difference between the data sets. The test decision for a normal distribution was obtained with the Lilliefors test. All the calculations were done in MATLAB ${ }^{\circledR}$.

A test with a p-value $p<0.05$ was called significant. If the p-value is smaller than 0.01 , the test outcome was declared as very significant.

\section{Methods}

Already in the 1980s, it was indicated that beat generation in a human heart is a chaotic process [11]. As the RR-time series is not constant over time, and irregular, it cannot be completely described by a linear approach [12]. Thus, there is an increasing interest in nonlinear methods and methods from chaos theory recently [13].

For all the algorithms in this section, the set of RRintervals $\left\{R R_{1}, \ldots, R R_{N}\right\}$ of length $N$ is denoted as

$$
X:=\{x(1), \ldots, x(N)\}=\left\{R R_{1}, \ldots, R R_{N}\right\} .
$$

\subsection{Correlation Dimension}

The first nonlinear measure, the Correlation Dimension (CD) assesses the fractal dimension of the system attractor in the reconstructed phase space, i. e., measures the complexity of the chaotic system [14].

The CD index is calculated based on an algorithm proposed by Grassberger and Procaccia [15]. In the first step, a phase space reconstruction is created by

$$
\begin{array}{r}
Y_{i}=\{x(i), x(i+\tau), \ldots, x(i+(m-1) \tau)\}, \\
i=1, \ldots, N-(m-1) \tau .
\end{array}
$$

Hereby, $m$ is the embedding dimension and $\tau$ is the delay time. The concatenation $Y$ is a $M \times m$ matrix with $M:=N-(m-1) \tau$ row vectors $Y_{i}$.

The CD is obtained by considering correlations between points on this attractor. Therefore, the correlation integral is defined as

$$
C(r):=\frac{2}{M(M-1)} \sum_{i=1}^{M-1} \sum_{j=i+1}^{M} \Theta\left(r-\left\|Y_{i}-Y_{j}\right\|\right),
$$


where $r$ is the radius and $\Theta$ denotes the Heaviside function

$$
\Theta(x):= \begin{cases}0, & x \leq 0 \\ 1, & x>0\end{cases}
$$

$\left\|Y_{i}-Y_{j}\right\|$ is the Euclidean distance between a pair of points within the attractor. Hence, the correlation function counts the number of distances closer than $r$, over the total number of distances. Obviously $C(r)$ has values between 0 and 1 . If $M$ is sufficiently large and $r$ is small enough, the following definition holds:

$$
\mathrm{CD}:=\lim _{r \rightarrow 0} \frac{\log C(r)}{\log r} .
$$

In this work, the delay time and the embedding dimension are chosen as $\tau=1$ and $m=22$, according to [16]. The last step is the design of the scaling region for the power law behavior. The approach of [16] is the following: Obtain $r_{\max }$ at $C\left(r_{\max }\right)=0.1$ and $r_{\min }=r_{0}+0.25\left(r_{\max }-r_{0}\right)$, where $r_{0}$ is the smallest radius. Since most of the subjects had nearly the same scaling region, parameters for $r_{\max }$ and $r_{\min }$, respectively, were fixed as $r_{\text {max }}=0.15$ and $r_{\text {min }}=0.06$. In Figure 2, the plot of the scaling region and the linear fit for a healthy subject is shown.

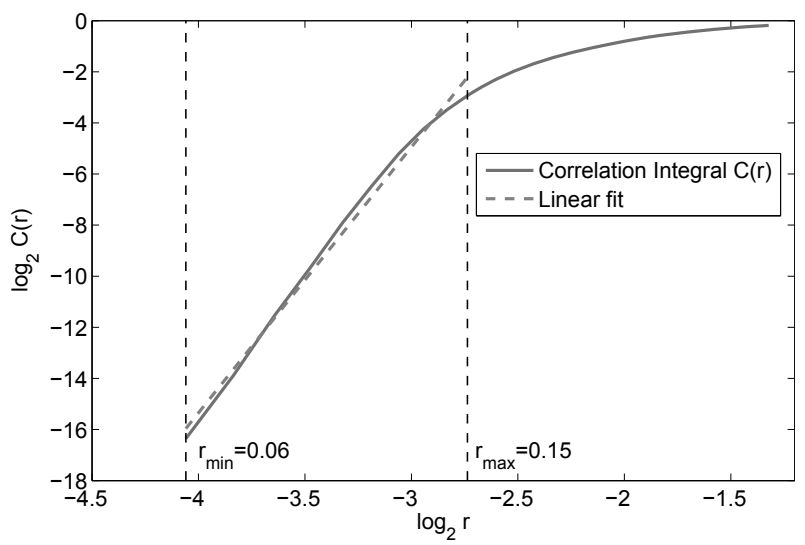

Figure 2: Correlation Dimension of a nonpathological subject. The fitting region lies between the black dashed lines.

\subsection{Fractal Dimension}

The term fractal is used for objects exhibiting a repeating pattern that displays at every scale. Famous examples of fractals are the Koch (Niels Fabian Helge von Koch (January 25, 1870 - March 11, 1924)) curve (also known as Koch snowflake), or the Sierpińksi (Wacław Franciszek Sierpiński (March 14, 1882 - October 21, 1969)) Triangle. For ordinary geometric objects, the Fractal Dimension (FD) equals the well known Euclidean or topological dimension, i.e., lines are onedimensional, surfaces have two dimensions and solids are three-dimensional. But the fractal dimension has not to be an integer.

To understand the need of the FD, the Koch curve is considered. Its topological dimension is one, but the length of the curve between two arbitrary points is infinite. One can assume that the curve can be explained as a fractal line, but it is too simple to be twodimensional [17]. The FD of the Koch curve is defined as FD $:=\frac{\log (4)}{\log (3)} \approx 1.26$, since in each step of scaling by $1 / 3$ the number of new sticks is equal to four. Indeed, the FD of the Koch curve lies between one and two. The first two iterations of the Koch curve are plotted in Figure 3.

Figure 3: The first two iterations of the Koch curve. In each iteration the lines are divided into three equal parts. The middle part is duplicated and then arranged like a triangle.

FD is calculated directly from the analyzed signal and has been applied to different biomedical signals, such as Electroencephalography (EEG) and ECG recordings [18].

The following algorithm was proposed by Higuchi in 1988 [19]:

1. For a fixed $k \in\left\{1, \ldots, k_{\max }\right\}$, construct $k$ new time series

$$
\begin{array}{r}
x_{m}^{k}=\{x(m), x(m+k), x(m+2 k), \ldots, x(m+\lfloor a\rfloor k)\} \\
m=1, \ldots, k,
\end{array}
$$

where $\lfloor a\rfloor:=\left\lfloor\frac{N-m}{k}\right\rfloor$.

2. Compute the length of each time series

$$
L_{m}(k)=\sum_{i=1}^{\lfloor a\rfloor}|x(m+i k)-x(m+(i-1) k)| .
$$


3. Normalize the lengths for each $k$

$$
\widetilde{L_{m}}(k)=L_{m}(k) \frac{N-1}{\lfloor a\rfloor k} .
$$

4. Calculate the average length

$$
L(k):=\operatorname{mean}_{m}\left(\widetilde{L_{m}}(k)\right) .
$$

Theses steps are repeated for $k=1, \ldots, k_{\max }$.

The FD is defined as the slope of the linear fit (in a least squares sense) of the plot $1 / \log (L(k))$ versus $\log (k)$. An example can be seen in Figure 4.

The implemented method is an adaption of Higuchi's algorithm with a moving time window of fixed window length. Hence, this method is sometimes specified as running FD [20].

According to [21], the parameter $k_{\max }$ was set to $k_{\max }=10$ and the RR-intervals were divided into windows with a length of 100 samples, shifted by one sample. The FD of one entire record was obtained by averaging all FDs of all windows.

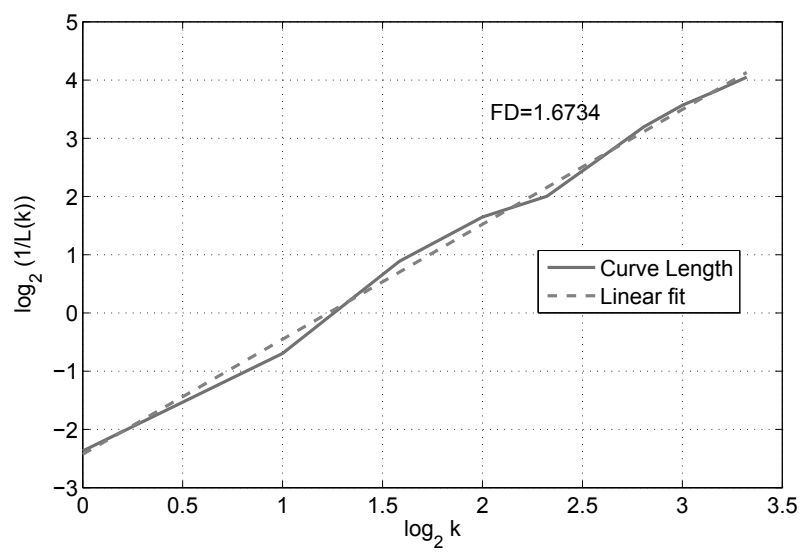

Figure 4: The Fractal Dimension calculated on a window of 100 samples of a nonpathological subject. $k_{\max }=10$

\section{Results}

In table 2, the distribution parameters of both indices are presented. Since the calculated values of the indices are normally distributed, the mean value, standard deviation and the corresponding p-value are listed.

Furthermore, Figure 5 shows boxplots of the measures calculated from the nonpathological and the pathological database, respectively. The left boxplot shows the CD with no significant difference between both groups, whereas the FD shows very significant differences between the tested groups.

\begin{tabular}{cccc}
\hline & Nonpath. Data & Path. Data & p-Value \\
\hline$C D$ & $7.25(2.42 \mathrm{SD})$ & $8.22(4.63 \mathrm{SD})$ & 0.26 \\
$F D$ & $1.68(0.14 \mathrm{SD})$ & $1.95(0.09 \mathrm{SD})$ & $<0.01$ \\
\hline
\end{tabular}

Table 2: Statistical parameters of the Correlation Dimension $C D$ and Fractal Dimension FD for the test. Mean and Standard Deviation (SD) are listed since data follows a normal distribution.

\section{Discussion}

The computed values of the CD are similar to the reported ones in [16]. Mia et al. characterized the HRV by means of the CD for two groups, i.e., healthy subjects and hypertension patients and distinguish between values during the day and at night. Their outcome was that healthy subjects had a lower CD in daytime than at night. This relationship was not seen in hypertensive patients [16]. Since the recording time was not specified for the pathological data and for the nonpathological database, this may have affected the results. The wide scatter indicates this effect.

Acharya et al. reported in [22] that the index CD varies for different kind of cardiac abnormalities. E. g., the $\mathrm{CD}$ calculated of subjects with atrial fibrillation is rather high compared to the $\mathrm{CD}$ value of patients where the heart rate remains stable (e. g., complete heart block).

Higuchi's Fractal dimension is very close to 2 for the pathological dataset. These values are also reported in [23]. The FD of healthy subjects is significantly smaller. The higher values of FD may result during ectopic beat variation in the heart rate.

\subsection{Limitations}

The outcome of this work is mainly bounded by two limitations. First, the composition of the pathological database is not as homogeneous as it should be. Precisely, subjects with different pathologies, including people with and without arrhythmias, are included.

Second, the absence of the recording time may yield comparing indices calculated on different day times. 
CD

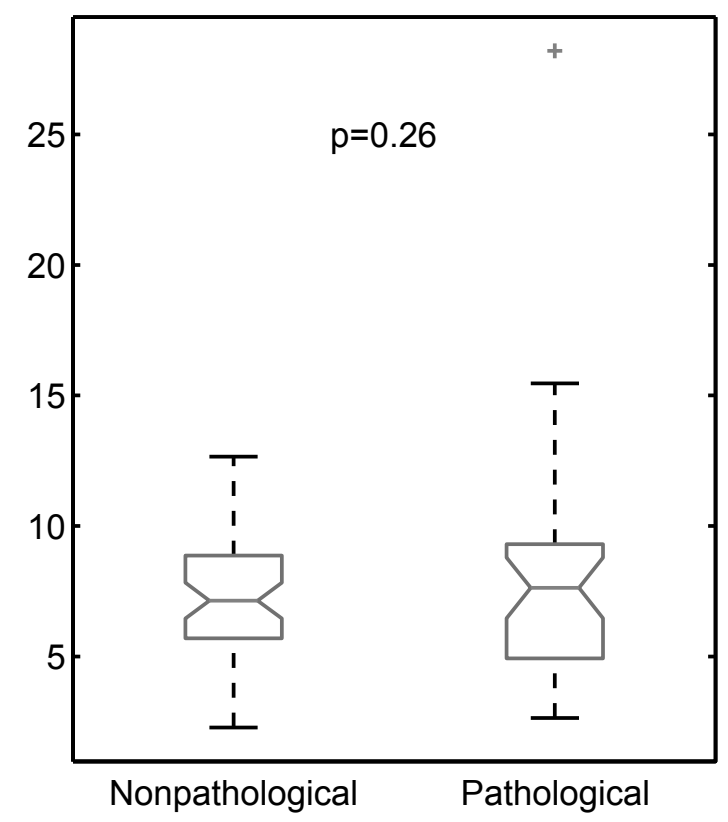

(a)
FD

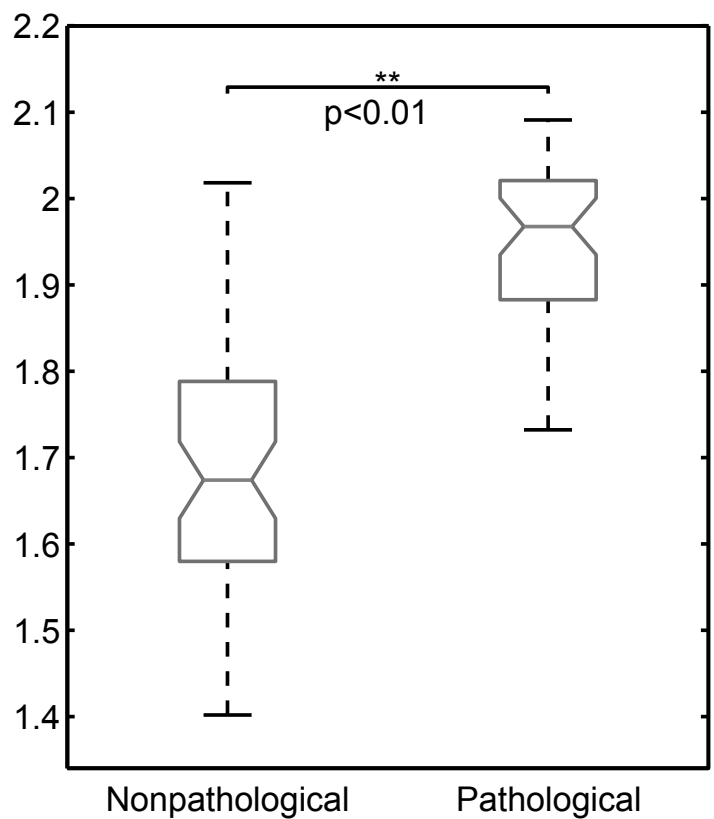

(b)

Figure 5: Boxplots for the test of the (a) Correlation Dimension index $C D$, (b) Fractal Dimension FD.

\section{Conclusion and Outlook}

Higuchi's FD is a fast and useful method to differentiate between nonpathological and pathological subjects. The CD was not able to asses, if a subject is healthy or not. Further examination of the design of the scaling region and a separation of day- and nighttime is suggested.

\section{References}

[1] Townsend N, Nichols M, Scarborough P, Rayner M. Cardiovascular disease in Europe-epidemiological update 2015. European heart journal. 2015; p. ehv428.

[2] Acharya UR, Joseph KP, Kannathal N, Lim CM, Suri JS. Heart rate variability: a review. Medical \& biological engineering \& computing. 2006; 44(12):1031-51.

[3] Cripps TR, Malik M, Farrell TG. Prognostic value of reduced heart rate variability after myocardial infarction: clinical evaluation of a new analysis method. British heart journal. 1991;65(1):14-19.
[4] Signorini M, Ferrario M, Marchetti M, Marseglia A. Nonlinear analysis of heart rate variability signal for the characterization of cardiac heart failure patients. In: Engineering in Medicine and Biology Society, 2006. EMBS'06. 28th Annual International Conference of the IEEE. IEEE. 2006; pp. 3431-3434.

[5] Akay M. Nonlinear Biomedical Signal Processing, Dynamic Analysis and Modeling, vol. I. New York, NY, USA: Wiley-IEEE Press. 2001.

[6] Goldberger AL, Amaral LAN, Glass L, Hausdorff JM, Ivanov PC, Mark RG, Mietus JE, Moody GB, Peng CK, Stanley HE. PhysioBank, PhysioToolkit, and PhysioNet: Components of a New Research Resource for Complex Physiologic Signals. Circulation. 2000; 101(23):e215-e220.

[7] Moody GB, Mark RG. The impact of the MIT-BIH arrhythmia database. IEEE Engineering in Medicine and Biology Magazine. 2001;20(3):45-50.

[8] Bauer A, Malik M, Schmidt G, Barthel P, Bonnemeier H, Cygankiewicz I, Guzik P, Lombardi F, Müller A, Oto A, Schneider R, Watanabe M, Wichterle D, Zareba W. Heart Rate Turbulence: Standards of Measurement, Physiological Interpretation, and Clinical Use. 
International Society for Holter and Noninvasive Electrophysiology Consensus. Journal of the American College of Cardiology. 2008;52(17):1353-1365.

[9] Xia L, Jing J. An Ensemble Density-based Clustering Method. Proceedings on Intelligent Systems and Knowledge Engineering (ISKE2007). 2007;

[10] Hörtenhuber M, Bachler M, Wassertheurer S, Mayer C. Comparison of Two Filtering Methods for Heart Rate Variability Analysis. SNE Simulation Notes Europe. 2014;24(3-4):137-142.

[11] Michaels DC, Chialvo DR, Matyas EP, Jalife J. Chaotic activity in a mathematical model of the vagally driven sinoatrial node. Circulation research. 1989; 65(5):1350-1360.

[12] Lombardi F. Chaos theory, heart rate variability, and arrhythmic mortality. Circulation. 2000;101(1):8-10.

[13] Huikuri HV, Mäkikallio TH, Perkiömäki J. Measurement of Heart Rate Variability by Methods Based on Nonlinear Dynamics. Journal of Electrocardiology. 2003;36(SUPPL.):95-99.

[14] Lombardi F, Sandrone G, Mortara A, Torzillo D, Rovere MTL, Signorini MG, Cerutti S, Malliani A. Linear and nonlinear dynamics of heart rate variability after acute myocardial infarction with normal and reduced left ventricular ejection fraction. The American Journal of Cardiology. 1996;77(15):1283-1288.

[15] Grassberger P, Procaccia I. Characterization of strange attractors. Physical review letters. 1983;50(5):346-349.

[16] Miao X, He W, Yang H, Tai HM. Heart rate variability characterization using correlation dimension. In:
Circuits and Systems, 2002. MWSCAS-2002. The 2002 45th Midwest Symposium on, vol. 1. IEEE. 2002; pp. I-447.

[17] Harte D. Multifractals, Theory and Applications. CRC Press. 2001.

[18] Esteller R, Member S, Vachtsevanos G, Member S, Echauz J, Litt B. A Comparison of Waveform Fractal Dimension Algorithms. IEEE Transactions on Circuits and Systems I: Fundamental Theory and Applications. 2001;48(2):177-183.

[19] Higuchi T. Approach to an irregular time series on the basis of the fractal theory. Physica D: Nonlinear Phenomena. 1988;31(2):277-283.

[20] Klonowski W. Application of nonlinear dynamics in biosignal analysis. Proceedings of SPIE - The International Society for Optical Engineering. 2005; 5975.

[21] Pierzchalski M, Stepien RA, Stepien P. New Nonlinear Methods of Heart Rate Variability Analysis in Diagnostics of Atrial Fibrillation. International Journal of Biology and Biomedical Engineering. 2011; 5(4):201-208.

[22] Acharya R, Lim C, Joseph P. Heart rate variability analysis using correlation dimension and detrended fluctuation analysis. Itmb-Rbm. 2002;23:333-339.

[23] Pierzchalski M. Assessing cardiovascular reaction to antiarrhythmic drugs using Higuchi's fractal dimension. In: Assessing cardiovascular reaction to antiarrhythmic drugs using Higuchi's fractal dimension. 2011; pp. 200-202. 


\title{
Reservoir Wave Paradigm: An Implementation and Sensitivity Analysis
}

\author{
Markus Ebner $^{1}$, Bernhard Hametner ${ }^{1,2 *}$, Stefanie Parragh ${ }^{1,2}$, Siegfried Wassertheurer ${ }^{2}$ \\ ${ }^{1}$ Department of Analysis and Scientific Computing, Vienna University of Technology, Wiedner Hauptstraße 8-10, \\ 1040 Vienna, Austria \\ ${ }^{2}$ Health and Environment Department, AIT Austrian Institute of Technology GmbH, Donau-City-Straße 1, 1220 \\ Vienna, Austria; *bernhard.hametner@ait.ac.at
}

Simulation Notes Europe SNE 25(3-4), 151 - 156

DOI: $10.11128 /$ sne.25.tn. 10307

Received: March 11, 2015; Revised September 25, 2015;

Accepted: October 11, 2015

Abstract. Striving for a better understanding of complex phenomena of hemodynamics and the need of reliable, clinically relevant information on the circulatory system are the driving forces to refine already existing cardiovascular models. A recent modelling theory is called the reservoir wave paradigm (RWP) and merges two existing models in order to address two important phenomena at the same time: arterial compliance and wave propagation. The aim of this article is to present its inherent concept and to illustrate its sensitivity to different model parameters. For this purpose the RWP is applied to an exemplary pressure waveform originating at the ascending aorta by using two different estimators of systolic time duration as well as two different choices of minimization intervals for the subsequent reservoir pressure fitting. The findings indicate a substantial impact of the chosen optimization interval. In contrast, the effect of the regarded estimators of systolic time duration on the waveform might be considered as negligible.

\section{Introduction}

Cardiovascular modelling plays a key role in order to quantify the physical state of the human circulatory system. In this context, the investigation of time and location dependent waveforms of arterial pressure and flow are of particular interest, since they are capable to provide indicators for cardiovascular diseases. For example arterial stiffness and wave reflection parameters have been revealed as clinically relevant predictors of risk and have been suggested to be incorporated into clinical practice [1-5].
To date various model concepts for describing the pressure and flow waveforms have been proposed. Beside the frequently used approaches of wave analysis, wave separation or the Windkessel method, a recently emerged theory is called the reservoir wave paradigm (RWP). Whereas the former methods concentrate on either the wave behaviour or the Windkessel behaviour, the RWP aims to incorporate both phenomena by separating the measured pressure waveform in an arterial compliance-related reservoir pressure and a waveassociated excess pressure [6]. At present the justification of this fairly new approach and its inherent assumptions are highly debated [7,8]. However, in this article it shall be focussed on the underlying model concept of the RWP and its sensitivity to the model parameters of systolic time duration and optimization intervals necessary to obtain the reservoir pressure.

The pressure separation according to the RWP can be performed in various ways depending on the availability of pressure and flow waveforms. In this work, the commonly used method of only using pressure curves is derived and applied to a pressure waveform originating at the ascending aorta. The underlying concept of fitting the reservoir pressure waveform to the measured one in later diastole requires an estimation of systolic time duration and a corresponding appropriate minimization interval. For this purpose, two different estimators of systolic time durations are presented and used to define two different optimization intervals. All methods are implemented in MATLAB and described step-by-step. Based on the results of this implementation, the impact of the chosen algorithm parameterization is discussed and analysed. 


\section{Methods}

\subsection{Reservoir Theory}

The RWP is based on the assumption that the actual pressure and flow waveforms are shaped by two prevailing phenomena within the arterial tree: the arterial compliance and wave propagation related phenomena [9].

The former describes the 'reservoir effect' of cushioning and recoiling of the major arteries, which is mainly determined by the ascending aorta as the most elastic part within the arterial tree [10]. This phenomenon is modelled by a classic two-element Windkessel concept [11].

The latter takes account for propagation phenomena of travelling waves produced by every heartbeat. Due to reflections, caused by impedance mismatches along the arterial tree, the actual waveforms can be regarded as superposition of forward and backward travelling waves. This phenomenon depends strongly on local properties.

Separation of pressure. According to the RWP, a reasonable ansatz is now to divide the actual pressure waveform $p$ along the arterial tree into an instantaneous sum of a time-dependent arterial compliance-related reservoir pressure $p_{\text {res }}$ and the remaining local, waveassociated pressure $p_{\text {ex }}$ depending on time $t$ and distance $x$ along the arteries [6], i.e.

$$
p(x, t)=p_{\text {res }}(t)+p_{\text {ex }}(x, t) .
$$

Hence, the compliance-related reservoir pressure is implicitly regarded as spatially uniform but shifted in time that it takes the waveform from the ascending aorta to arrive at the regarded vessel. Therefore, the observable differences in pressure waveforms along the arterial tree shall be fully described by the local excess pressure according to the RWP [12].

The actual pressure separation (1) is performed by computing the reservoir pressure $p_{\text {res }}$ from a given pressure waveform $p$ which then defines the remaining excess pressure $p_{\text {ex }}$.

Reservoir pressure based on flow. The reservoir effect is related to the change of volume in time within the arterial tree and leads to the classic two-element Windkessel equation

$$
\frac{d\left(p_{\text {res }}-P_{\infty}\right)}{d t}(t)=\frac{p_{\text {res }}(t)-P_{\infty}}{R C}-\frac{q_{\text {in }}(t)}{C}
$$

where $P_{\infty}$ denotes the asymptotic pressure, $R$ the arterial resistance, $C$ the arterial compliance and $q_{\text {in }}$ the flow into the arterial tree right after the left ventricle [6]. It shall be assumed that $0 \leq t \leq T_{b}$ describes one heartbeat with time duration $T_{b}$ from the beginning of systole $(t=0)$ to the end of diastole $\left(t=T_{b}\right)$. The time $t=T_{s}$ with $0<T_{s}<T_{b}$ refers to the closure of the aortic valve and shall indicate the transition from systole to diastole. From this time the flow $q_{\text {in }}$ is approximately zero and the wave activity decreases, leading to a negligible excess pressure at the end of diastole. Thus, for given parameters $R, C$ and $P_{\infty}$ the unique solution of (2) is defined by the initial value $p_{\text {res }}(0)=p(0)$ and reads

$$
\begin{aligned}
p_{\text {res }}(t)= & \frac{e^{-t /(R C)}}{C} \int_{0}^{t} q_{\text {in }}(s) e^{s /(R C)} d s \\
& +\left(p(0)-P_{\infty}\right) e^{-t /(R C)}+P_{\infty}
\end{aligned}
$$

for $0 \leq t \leq T_{b}$. By assuming the ventricular flow $q_{i n}$ to be zero during diastole, the solution is given by

$$
p_{\text {res }}(t)=\left(p_{\text {res }}\left(T_{s}\right)-P_{\infty}\right) e^{-\left(t-T_{s}\right) /(R C)}+P_{\infty}
$$

for $T_{s} \leq t \leq T_{b}$. Hence, provided that a simultaneously measured flow $q_{i n}$ and an estimation of $T_{s}$ are available, one might fit the reservoir pressure (4) to the measured pressure $p$ in diastole in order to get approximations on the asymptotic pressure $P_{\infty}$ and the time constant $\tau:=R C$ in the first place. A subsequent minimization of the difference between the reservoir pressures (3) and (4) at $t=T_{s}$ yields the remaining parameters of $R$ and $C$. Consequently, the reservoir pressure $p_{\text {res }}(t)$ is fully described by (3) for all $0 \leq t \leq T_{b}$.

The procedure described above was essentially done in [6], which revealed an excess pressure approximately proportional to the flow in the ascending aorta, i.e.

$$
q_{\text {in }}=\zeta p_{\text {ex }}=\zeta\left(p-p_{\text {res }}\right)
$$

with $\zeta>0$. The proportionality (5) basically states Ohm's law in hydraulic analogy and was one of the key findings of the RWP. In further consequence, the observation (5) became a crucial assumption and led to the elimination of the constraining requirement of flow knowledge.

Reservoir pressure without flow. By assuming the relationship (5) and inserting it into (2) one obtains the ordinary differential equation

$$
\begin{gathered}
\frac{d\left(p_{\text {res }}-P_{\infty}\right)}{d t}(t)+(a+b)\left(p_{\text {res }}(t)-P_{\infty}\right) \\
=a\left(p(t)-P_{\infty}\right)
\end{gathered}
$$


with the rate constants $a=\zeta / C$ and $b=1 /(R C)$ at the ascending aorta. Both rate constants have the same SIunit: $1 /$ s. Similarly to (3), the solution of (6) reads

$$
\begin{array}{r}
p_{\text {res }}(t)=e^{-(a+b) t} \int_{0}^{t} a\left(p(s)-P_{\infty}\right) e^{(a+b) s} d s \\
+\left(p(0)-P_{\infty}\right) e^{-(a+b) t}+P_{\infty}
\end{array}
$$

for $0 \leq t \leq T_{b}$ whereby the approximated exponential decay (4) in diastole corresponds to

$$
p_{\text {res }}(t)=\left(p_{\text {res }}\left(T_{s}\right)-P_{\infty}\right) e^{-b\left(t-T_{S}\right)}+P_{\infty}
$$

in the new variables for $T_{s} \leq t \leq T_{b}$.

In the following the pressure separation (1) via (7) and ( 8 ) as described above shall be implemented. Moreover, two different estimations of the time duration of systole $T_{S}$ by the mere knowledge of the pressure waveform will be presented.

\subsection{Data}

The investigated pressure curve is taken from [13], which in turn references to [14], and illustrates a pressure waveform of one heart cycle at the ascending aorta. It is given in terms of the arrays of pressure $p$ (in unit $\mathrm{mmHg}$ ) and time $t$ (in s). The sampling rate of the pressure curve is $0.0078 \mathrm{~s}$, which corresponds to a sampling frequency of $128 \mathrm{~Hz}$. Due to the digitized nature of the data, all operations shall be understood as discrete ones in the following.

\subsection{Duration of systole}

Two estimation methods of the duration of systole are used in this article. The first is based on the maximum curvature of the pressure $p$ and is computed by

$$
T_{s, c}:=\underset{t^{\prime}}{\operatorname{argmax}}\left(\frac{d^{2} p}{d t^{2}}\left(t^{\prime}\right)\right)
$$

with $t^{\prime}$ being within a range around an initial guess of $T_{S}$.

The second method relies on the minimum derivation of the pressure $p$ and is defined by

$$
T_{s, d}:=\underset{t^{\prime}}{\operatorname{argmin}}\left(\frac{d p}{d t}\left(t^{\prime}\right)\right)
$$

with $0 \leq t^{\prime} \leq T_{b}$.

\subsection{Minimization interval for reservoir curve fitting}

The idea is to fit the reservoir curve (8) to the measured one such that $p_{\text {res }}$ closely aligns $p$ when wave activity is believed to be minimal. It is common to consider approximately the last two thirds of diastole for this purpose $[6,15]$, i.e. $T_{s}+\left(T_{b}-T_{s}\right) / 3 \leq t \leq T_{b}$.
However, in literature one may also find a fitting over the whole diastole [12]. Therefore, both intervals will be regarded in the comparison.

\subsection{Implementation of the RWP}

The implementation of the RWP can be done in several ways. The one presented in here basically follows the explanations in [15]:

1. Determine the duration $T_{b}$ of the given heart beat.

2. Estimate the duration of systole $T_{S}$ by means of (9) or (10).

3. Fit the reservoir pressure to the measured curve by using the assumed exponential decay (8) of $p_{\text {res }}$ during diastole. Therefore, find $b, p_{\text {res }}\left(T_{*}\right), P_{\infty}>0$ such that

$$
\left\|p_{\text {res }}-p\right\|_{\ell^{2}\left(T_{*}, T_{b}\right)} \rightarrow \min
$$

whereby $T_{*}$ denotes the start of the minimization interval in diastole. Thus, $T_{*}$ is meant to correspond to either $T_{s}$ or $T_{s}+\left(T_{b}-T_{s}\right) / 3$ associated with the respective systolic durations of (9) and (10). The minimization (11) is realized via the MATLAB function lsqnonlin. The initial parameters for the optimization are set to

$$
b_{0}:=3 \cdot 1 / \mathrm{s}, \quad p_{\text {res }}\left(T_{*}\right)_{0}:=p\left(T_{*}\right), P_{\infty, 0}:=\min p
$$

with the constraint that $30 \mathrm{mmHg} \leq P_{\infty} \leq P_{\infty, 0}$.

4. The remaining rate constant $a$ is determined by seeking continuity at $T_{*}$ between the piecewisely defined reservoir curve via (7) in systole and (8) in diastole. Hence, find $a>0$ such that their absolute difference is minimal at the transition time $t=T_{*}$. In MATLAB the optimization is done by using again lsqnonlin with initial value $a_{0}:=15 \cdot 1 / \mathrm{s}$, whereby the integral in (7) is implemented via the function trapz.

\section{Results}

In this section the obtained pressure separation according to the algorithm above is illustrated. Moreover, the effects of the choice of estimated systolic duration and minimization interval on the RWP results are presented.

\subsection{Separation of pressure}

In Figure 1 the pressure separation based on the estimated systolic duration $T_{s, d}$ and the use of the whole diastole for reservoir curve fitting is shown. 
For illustration purposes the diastolic blood pressure (DBP), defined as the minimum of the waveform $p$, was subtracted from the reservoir and measured pressure curves. It can be witnessed that in early systole the excess pressure $p_{e x}$ is a good approximation of the measured pressure $p$. However, after reaching its peak it declines quickly and remains approximately zero during diastole. As indicated in [6] the waveform of $p_{\text {ex }}$ is suggested to be a good approximation to the flow $q_{\text {in }}$ in the ascending aorta. In contrast, the reservoir pressure $p_{\text {res }}$ increases gradually during systole and hits its peak in late systole where it then closely aligns the measured pressure waveform.

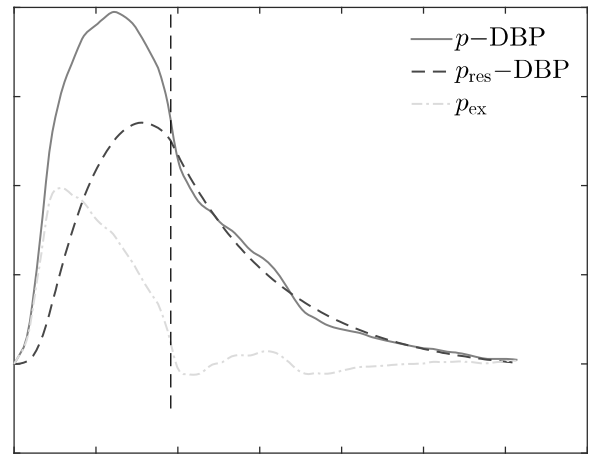

Figure 1. Pressure separation according to the RWP. For illustration purposes the DBP $=84.56 \mathrm{mmHg}$ was subtracted from both the reservoir and measured pressure waveform. The dashed vertical line indicates the estimated systolic duration $T_{s, d}=0.38 \mathrm{~s}$.

\subsection{Sensitivity on minimization interval}

In Figure 2 the reservoir curves $p_{\text {res }}(2 / 3 D, d)$ and $p_{\text {res }}(D, d)$ represent the waveforms obtained by either using two-thirds or the whole diastole associated with the estimated time $T_{s, d}$, respectively. The vertical dashdotted line indicates $t=T_{s, d}$ and the vertical dotted line the time $t=T_{s}+\left(T_{b}-T_{s}\right) / 3$, referring to the beginning of the last two-thirds of diastole. It can be observed that the later start of the optimization interval leads to a higher peak in the reservoir waveform. Since the information of the prior pressure decay in the first third is not taken into account its corresponding reservoir pressure is only determined by a small range of $p$-values at later diastole. In contrast, the reservoir waveform $p_{\text {res }}(D, d)$ is computed by a broader range of $p$, which enables a closer matching of $p_{\text {res }}$ to the actual measured pressure decay.

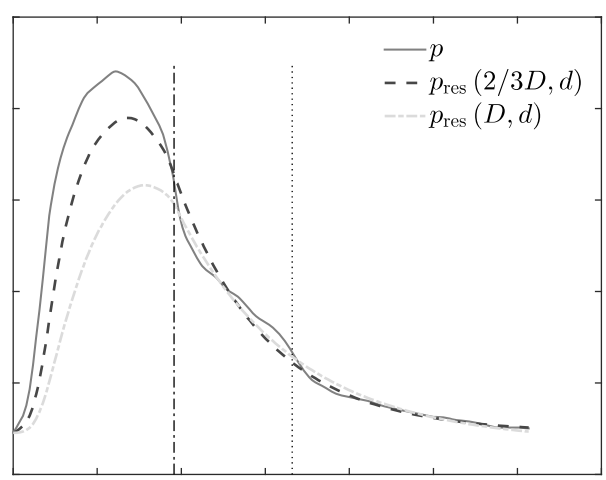

Figure 2. Reservoir pressures based on the estimation $T_{s, d}$ (vertical dash-dotted line) and its associated two-thirds of diastole $\left(p_{\text {res }}(2 / 3 D, d)\right)$ and its entire diastolic duration $\left(p_{\text {res }}(D, d)\right)$. The vertical dotted line indicates the beginning of the last two-thirds of diastole.

In Table 1 a more detailed analysis and comparison of both approaches are provided. The pulse pressure (PP) is defined as the difference between the maximum and minimum pressure value. The pressure area (A) is computed as the integral of the respective pressure curve subtracted by the DBP. By taking the reservoir waveform $p_{\text {res }}(D, d)$ as the reference the relative differences of about $27 \%$ in PP and almost $22 \%$ in area quantify the already witnessed discrepancies. The figures of both asymptotic pressures $P_{\infty}$ are close to the upper limit of the DBP $=84.56 \mathrm{mmHg}$ as a consequence of the smooth decay of $p$ at the end of diastole.

\begin{tabular}{ccccc}
\hline & 2/3D,d & D,d & abs, diff. & rel. diff. [\%] \\
\hline PP [mmHg] & 34.42 & 27.06 & 7.37 & 27.22 \\
\hline A [mmHg s] & 15.10 & 12.42 & 2.68 & 21.58 \\
\hline $\boldsymbol{P}_{\infty}$ [mmHg] & 84.56 & 83.46 & 1.09 & 1.31 \\
\hline \multicolumn{4}{c}{ Table 1. Overview of the pulse pressures (PP), areas (A) } \\
and asymptotic pressures $P_{\infty}$ of the waveforms \\
$p_{\text {res }}(2 / 3 D, d)$ and $p_{\text {res }}(D, d)$, respectively.
\end{tabular}

Based on the computed reservoir pressures of the respective minimization intervals, the results of the corresponding excess pressures $p_{\text {ex }}(2 / 3 D, d)$ and $p_{\text {ex }}(D, d)$ are presented in Figure 3 . Since generally $p_{\text {res }}(2 /$ $3 D, d) \geq p_{\text {res }}(D, d)$ during systole, it holds that $p_{\text {ex }}(2 / 3 D, d) \leq p_{\text {ex }}(D, d)$ in this period of time according to (1). 


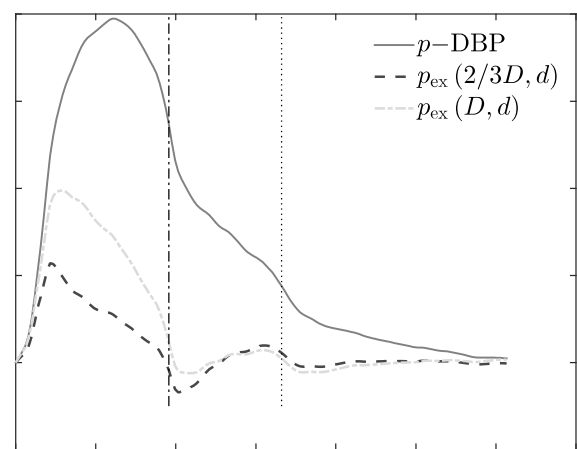

Figure 3. Excess pressures based on the estimation $T_{s, d}$ (vertical dash-dotted line) and its associated two-thirds of diastole $\left(p_{e x}(2 / 3 D, d)\right)$ and its entire diastolic duration $\left(p_{e x}(D, d)\right)$. The vertical dotted line indicates the beginning of the last two-thirds of diastole.

\subsection{Sensitivity on estimation of systolic duration}

Figure 4 shows the respective reservoir waveforms obtained by minimizing the differences of (8) and the measured waveform $p$ in entire diastole associated with both estimations $T_{s, c}$ and $T_{s, d}$. It holds that $T_{s, c}>T_{s, d}$ and that the pulse pressure of $p_{\text {res }}(D, d)$ is slightly higher during systole but no substantial differences in reservoir waveforms are observable.

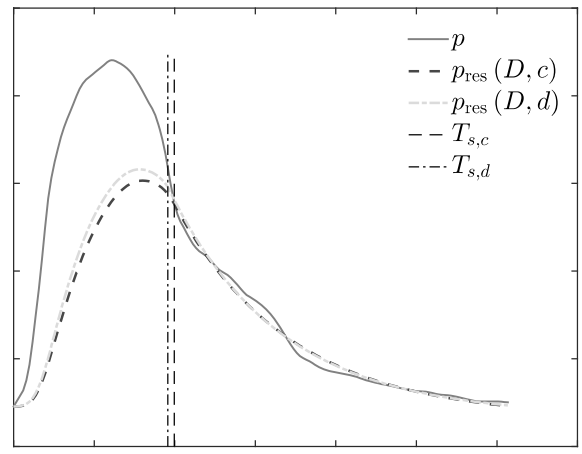

Figure 4. Reservoir pressures based on the minimization along entire diastole based on different estimations of systolic duration $T_{s, c}$ $\left(p_{\text {res }}(D, c)\right)$ and $T_{s, d}\left(p_{\text {res }}(D, d)\right)$.

Table 2 enables a more detailed discussion of the differences: The estimated systolic duration $T_{s, c}$ based on the maximum curvature of $p$ is approximately $0.02 \mathrm{~s}$ longer compared to its counterpart $T_{s, d}$ which relies on the minimum derivative.
Furthermore, only slight discrepancies are visible in terms of pulse pressure and area. The reservoir waveforms related to $T_{s, c}$ are about $-5 \%$ and approximately $4 \%$ smaller in pulse pressure and in area than their $T_{s, d^{-}}$ counterparts. Additionally, only minor differences are noticeable between their asymptotic pressures too whereby the longer time $T_{S, c}$ yields a slightly lower asymptotic pressure.

\begin{tabular}{lcccc}
\hline & $T_{s, c}$ & $T_{s, d}$ & abs. diff. & rel. diff. [\%] \\
\hline $\boldsymbol{T}_{s}$ [s] & 0.40 & 0.38 & 0.02 & 4.08 \\
\hline PP [mmHg] & 25.78 & 27.06 & -1.28 & -4.72 \\
\hline A [mmHg s] & 11.98 & 12.42 & -0.44 & -3.57 \\
\hline $\boldsymbol{P}_{\infty}[\mathrm{mmHg}]$ & 83.22 & 83.46 & -0.24 & -0.29 \\
\hline
\end{tabular}

Table 2. Overview of the pulse pressures (PP), areas (A) and asymptotic pressures $P_{\infty}$ of the waveforms $p_{\text {res }}(D, c)$ and $p_{\text {res }}(D, d)$, respectively.

\section{Discussion}

In this work, the concept of the RWP as well as a possible implementation and its sensitivity to the numerical realization were presented. The theoretical concept of pressure separation according to the RWP might seem natural due to the mentioned phenomena of aortic compliance and wave propagation, which can also be seen in the results shown in Figure 1. The reservoir pressure represents the cushioning and recoiling effect which smooths the pulsatile pressure and flow of every cardiac ejection: During the ejection period the left ventricle pumps blood into the arterial system where the 'blood storage' gets filled steadily. After closure of the aortic valves the ascending aorta forwards the blood and, thus, enables a perfusion at tissue level throughout the cardiac cycle.

Nevertheless, a concise definition of the application of this concept onto a pressure waveform is difficult to state. Aguado-Sierra et al. [15] for example use approximately the last two thirds of diastole to fit the reservoir pressure (8) to the actual pressure waveform. Parker et al. [12] use (10) as $T_{s}$-estimator and fit the assumed exponential decay of the reservoir pressure during the whole diastole. Thereafter, they choose the first crossover point of the resulting curves (7) and (8) as transition to define the entire reservoir curve. 
Additionally, the MATLAB function fminsearch is taken for the minimization itself. Vermeersch et al. [16] primarily use the method as described in [12], but switch to the last two thirds for curve fitting when the procedure is not successful for entire diastole. Moreover, they assume the asymptotic pressure $P_{\infty}$ to be zero.

Apart from that, the optimization criteria could be adapted. Other norms as the $\ell^{2}$-norm used in here are possible and different parameter ranges may be permitted, including different initial values and boundary conditions [17].

Consequently, one may find different suggestions of the RWP implementation in literature which, in turn, are based on the set of available test curves. The results presented in this work indicate a substantial impact of the minimization interval on the reservoir pressure waveform. The reservoir curves related to the two regarded different optimization intervals show remarkable differences of almost $30 \%$ in their corresponding reservoir pulse pressures. In contrast, the differences caused in asymptotic pressures may be considered as negligible. In this context, one should bear in mind that the obtained figures depend strongly on the underlying exemplary pressure curve and might differ markedly to other ones.

In conclusion, whereas the actual discussion regarding scrutiny of the RWP mainly focuses on its justification related to physiological aspects, the results presented in this work also suggest a considerable sensitivity associated with the numerical and algorithmic implementation. Consequently, a broad consensus about the algorithms used is needed before the RWP can be incorporated into clinical practice.

\section{References}

[1] Mancia G, De Backer G, Dominiczak A, et al. Guidelines for the management of arterial hypertension. Eur Heart J. 2007; 28: 1462-536.

[2] Laurent S, Cockcroft J, Van Bortel L, Boutouyrie P, Giannattasio C, Hayoz D, Pannier B, Vlachopoulos C, Wilkinson I, Struijker-Boudier H. Expert consensus document on arterial stiffness: methodological issues and clinical applications. Eur Heart J. 2006; 27(21): 2588605.

[3] Hametner B, Wassertheurer S, Hughes A, Parker K, Weber T, Eber B. Reservoir and excess pressures predict cardiovascular events in high-risk patients. Int J Cardiol. 2014; 171: 31-6.
[4] Weber T, Wassertheurer S, Rammer M, Haiden A, Hametner B, Eber B. Wave Reflections, Assessed With a Novel Method for Pulse Wave Separation, Are Associated With End-Organ Damage and Clinical Outcomes. Hypertension. 2012; 60: 534-41.

[5] Weber T, Wassertheurer S, Hametner B, Parragh S, Eber B. Noninvasive methods to assess pulse wave velocity: comparison with the invasive gold standard and relationship with organ damage. J Hypertens. 2015; in press. http://dx.doi.org/10.1097/HJH.0000000000000518.

[6] Wang JJ, O'Brien AB, Shrive NG, Parker KH, Tyberg JV. Time-domain representation of ventricular-arterial coupling as a windkessel and wave system. Am J Physiol Heart Circ. 2003; 284(4): H1358-68.

[7] Westerhof N, Westerhof BE. The reservoir wave paradigm discussion. J Hypertens. 2015; 33(3): 458-60.

[8] Mynard JP, Smolich JJ, Avolio A. The ebbing tide of the reservoir-wave model. J Hypertens. 2015; 33(3): 461-4.

[9] Davies JE, Hadjiloizou N, Leibovich D, Malaweera A, Alastruey-Arimon J, Whinnett ZI, Manisty CH, Francis DP, Aguado-Sierra J, Foale RA, Malik IS, Parker KH, Mayet J, Hughes AD. Importance of the aortic reservoir in determining the shape of the arterial pressure waveform - The forgotten lessons of Frank. Artery Res. 2007; 1(2): 40-5.

[10] Nichols W, O'Rourke M, Vlachopoulos C. McDonald's blood flow in arteries. London: Hodder Arnold; 2011. $6^{\text {th }}$ ed.

[11] Westerhof N, Lankhaar J, Westerhof B. The arterial Windkessel. Med Biol Eng Comput. 2009; 47(2): 131-41.

[12] Parker KH, Alastruey J, Stan GB. Arterial reservoirexcess pressure and ventricular work. Med Biol Eng Comput. 2012; 50(4): 419-24.

[13] Johnson DA, Spaeth JR, Rose WC, Naik UP, Beris AN. An impedance model for blood flow in the human arterial system, Part I: Model development and MATLAB implementation. Comput Chem Eng. 2011; 35(7): 1304-16.

[14] Olufsen MS. Modeling flow and pressure in the systemic arteries. In: Ottesen JT, Olufsen MS, Larsen JK, editors. Applied Mathematical Models in Human Physiology. Philadelphia: SIAM; 2004.

[15] Aguado-Sierra J, Alastruey J, Wang JJ, Hadjiloizou N, Davies J, Parker KH. Separation of the reservoir and wave pressure and velocity from measurements at an arbitrary location in arteries. Proc Inst Mech Eng H. 2008; 222(4): 403-16.

[16] Vermeersch SJ, Rietzschel ER, De Buyzere ML, Van Bortel LM, Gillebert TC, Verdonck PR, Segers P. The reservoir pressure concept: The 3-element windkessel model revisited? Application to the Asklepios population study. J Eng Math, 2009; 64: 417-28.

[17] Ebner M. Reservoir Theory and its Application on Peripheral Arteries [Master thesis]. [Institute for Analysis and Scientific Computing, (AT)], Vienna University of Technology; 2014. 


\title{
Learning the Structure of Graphical Models Based on Discrete Time Series Data in the Context of Ambient Assisted Living
}

\author{
Sarah Fallmann ${ }^{1,2 *}$, Johannes Kropf ${ }^{2}$ \\ ${ }^{1}$ Department of Analysis and Scientific Computing, Vienna University of Technology, Wiedner Haupstraße 8-10, \\ 1040 Vienna, Austria; \\ ${ }^{2}$ Health \& Environment Department, AIT Austrian Institute of Technology GmbH, Wiener Neustadt, Austria \\ *sarah.fallmann.fl@ait.ac.at
}

Simulation Notes Europe SNE 25(3-4), 2015, 157-164 DOI: $10.11128 /$ sne. 25. tn. 10308

Received: June 16, 2015; Revised September 10, 2015; Accepted: October 5, 2015

\begin{abstract}
This work addresses the issue of building a probabilistic system in an ambient assisted living environment to ensure a proper living for older adults. The focus lies on the early prediction of human activities based on domotic sensor data which form a temporalsequential data set. In contrast to commonly used methods in sequential data mining, data in hidden streams and with variable temporal spans are considered. The aim in this context is to detect recurrent patterns in a stream of domestic sensor data using the TemporalPattern (T-Pattern) algorithm and to automatically generate probabilistic finite-state automata.
\end{abstract}

\section{Introduction}

The motivation of the work presented here is mainly driven by the demographic change and the need for ambient assisted technologies to support a longer independent living of older adults. Behavior pattern recognition based on non-obtrusive sensors is a challenging task, but is seen to be of fundamental importance for AAL technologies being accepted by the end users.[1] $\mathrm{Hu}-$ man behavior can be very complex, consider, for instance, the preparation of a meal. This activity consist of many other sub activities like taking the pan out of the cupboard, taking food out of the refrigerator, switching on the stove and so on.
The current attempts to detect human behavior and activity can be classified by the type of the sensors used (1) body worn sensors, (2) video cameras and (3) domestic sensor networks. For the sake of maximal nonobtrusiveness, this work concentrates on domotic sensor networks. The data gained from domotic sensors are mostly binary and form sequential patterns. Sequential pattern mining, which discovers frequent subsequences as patterns in such a data source, is an important problem with broad applications. This includes the analysis of customer purchases, natural disasters, disease treatment, DNA sequences and others.[2]

In the literature, methods for predicting the next symbol of a sequence of strings are proposed using probabilistic suffix trees (PST) [3, 4], which usually ignore the time between subsequent events and assume no noise in terms of unknown or random events in the data. In sensor networks consisting of many sensors, or in environments with multiple persons interacting with the smart home, patterns are very often hidden in data streams and must be discovered with appropriate statistical methods. Later the probabilistic suffix tree is transformed in an automaton. The automaton cannot be learned straight forward with this kind of data, as there exists no trainings data. This is the reason why PST are used to solve this problem.

There is a demand for future smart home solutions which increase the comfort and safety of the inhabitants while saving energy at the same time. The focus lies on caring for older adults, which are independent enough to live on their own, but desire to feel more secure. The security factor comprises, among other things, the sending of help in case of an accident or warning from an open window. 
This factors are treated in Ambient Assisted Living (AAL) technologies, in specific, behavior pattern recognition can handle and meet the request of older adults.[5] Nowadays state-of-the-art smart home solutions are based on simple predefined rules to control devices based on movement detectors or timers, but there is still very little intelligence built-in due to the following reasons:

- Too many systems on the market are proprietary and cannot be connected to each other

- Very few smart appliances are available on the market

- Living environments differ significantly and standardized solutions will not work

- Configuration is complex and therefore costly

One of the keys towards smart systems in the AAL context is seen to be activity recognition, and much research in recent years has focused on this topic. Although many approaches and algorithms exist, there are still many obstacles towards a system which is ready to be used in real environments in everyday life.

The aim of this work is to describe a concept for human behavior detection, which includes also the time aspect of environmental sensor events hidden in data streams. The time aspect is important, as data comes from real world settings and different sensors can send at similar timestamps. Moreover the order of the activities is important, in particular for events which are dependent on past events. The detection of high complex patterns and behaviors are important for a higher comfort of the end users.

\section{Related Work}

Human activity recognition is a challenging task and has been an active, fast growing and broad research area, with a multitude of approaches and algorithms proposed. Roughly, these approaches can be categorized by camera-based, environmental sensor-based, or wearable-/object-based activity recognition. This work focuses on non-obtrusive environmental sensors for activity recognition. Algorithms used in activity recognition can be divided into two major groups. The first one is based on machine learning techniques including supervised and unsupervised learning methods, the second one is based on logical modeling and reasoning.[1]
For probabilistic activity recognition a wide range of algorithms exists. This includes Hidden Markov Models (HMMs) [6], Bayes networks [7], decision trees and other classical pattern recognition methods. Among these, HMMs and Bayes networks are the most popular. These algorithms used in a supervised context are methods, which need annotated data to construct a model. On the other hand there exists unsupervised learning methods like factor analysis and mixture of Gaussians, these are learning methods without the need of preprocessing the data. These methods try to build statistical representations of the data.[8]

An approach similar to Hidden Markov Models is that of probabilistic finite state automata (PFA).[9] PFAs describe distributions over strings, but have been successfully used in several fields, including pattern recognition. Finite state machines in general are widely used for all kind of control tasks appearing in smart environments as well. The PFA is learned over a PST, where the nodes are the patterns from the T-Pattern algorithm.

PSTs are used in a wide area, for example to analyze video data [3]. Li et al. [3] used an action-only prediction model to represent dependencies between action units on the basis of video data. In comparison in the approach of this paper domestic sensors are used.

In the action-only prediction model, the occurrence of a sequence of events is used and compared with an empirical parameter to detect patterns. Another approach for the detection of patterns in behavior are $\mathrm{T}$ Patterns [10]. T-Patterns are created for finding hidden or non-obvious temporal patterns in streams of data describing human behavior. T-Patterns are originally used for children interaction analysis [10], but find also application in detecting animal and human behavior [11].

\section{Activity Recognition}

The sensors used for behavior detection are off-theshelf and low-cost conventional home automation sensors. All sensor types have been integrated in the platform and work in parallel due to the harmonization of data packets into a hardware abstraction bundle.[12]

For evaluation, three annotated datasets consisting of several weeks of data recorded in a real-world setting provided by Kasteren et al. [13] are used. Each dataset belongs to one house which is occupied by one person. 


\subsection{Algorithm}

The algorithm employed relies on the work of Li et al. [3] and their action-only prediction model. The authors try to construct a PST out of video data. This is done in four steps. First, they find every pattern in the video data and filter out the patterns which are not frequent. The frequent patterns are broken down into individual interactions along the time line. In the last step they calculate the probabilities of the various patterns with an algorithm containing some empirical parameters.

This fits the aim of this work quite well, but it must be expanded by the important temporal aspect. This is done by following the concept in Figure 1. In contrast to the algorithm proposed in [3], which only counts the occurrences of different frequent patterns, in this work the temporal aspect of the sensor data, as well as its statistical significance, is included in the calculation of the T-Pattern algorithm and the next symbol's probability to make it more robust. Another drawback of the referenced paper are the empirical parameters, which are unpractical and a stumbling block to a fully automatic system. In the present work, this problem does not occur.

If only the significant patterns are used to calculate the probabilities, a more appropriate result can be expected.

\subsection{Concept}

The real environmental sequential data from the senors are preprocessed. In this step false data are deleted and with this preprocessed data the algorithm can be proceeded.

First a T-Pattern algorithm is performed with the sensor data. Followed by building a PST with the significant T-Patterns. Therefore each significant T-Pattern stands for one node and the next symbol probabilities are calculated with the Poisson distribution.

The concept how to construct the next symbol probability of states in a PST relies on the idea of maximum duration compare Figure 1. This means in every pattern-step the longest duration of the patterns in this period is used to calculate the next symbol probability for this transition.

If the maximum duration of pattern-step 11 is interesting, the calculation begins at the pattern @ tt. The pattern-step 11 in this example means the transformation of @ tt in @ ttt, @ttk or @ ttx. The duration of every suffix of the pattern @ $\mathrm{tt}\{\mathrm{t}, \mathrm{k}, \mathrm{x}\}$ is compared and the

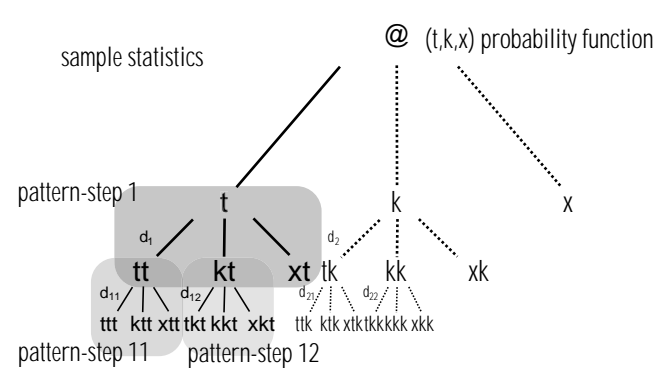

Figure 1: Concept how to calculate next symbol probability.

maximum duration is chosen to calculate the next symbol probability. All transformation probabilities, which are calculated in the way described before, are put to the correct node, where $x$ describes those cases where nothing significant happens. Therefore is it ensured that the total cases in one step sums always up to one. This is important, as otherwise no automaton can be constructed out of the PST.

To complete the system, the PST must be transformed in a probabilistic suffix automaton (PSA). In [14] an algorithm can be found for this step, the result for one example can be seen in Figure 2. The PSA is created by the information of the PST in the following way. First all leaves are added to the new automaton as recurrent states and a state equal to the root of the PST. The states are connected with each other. For example if the path between root and '021' is questioned, two states are required and connected '20' and ' 2 '.

In the next step the arcs are built, for this step the next-symbol probabilities of the PST are required. If there exists a next symbol probability after the given state, the symbol is added, and from the front symbol by symbol is removed until this state can be found in the automaton. For example '20' has a next symbol probability to ' 1 ', the constructed label is '201' after removing the first symbol ' 2 ' an already existing state ' 01 ' is found and an arc is created.

The last step is to assign state types. This is done by looking at each node and ascertaining if one of the arcs comes from a recurrent state created in step one, therefore this state also becomes a recurrent state. All other nodes are transient states. ' 2 ' is a recurrent state, as their exists an arc from the recurrent state '10' to '2'.[14]

This algorithm leads to a PSA, out of significant TPattern which can detect behavioral anomalies in real environments with domestic sensors.[12] 

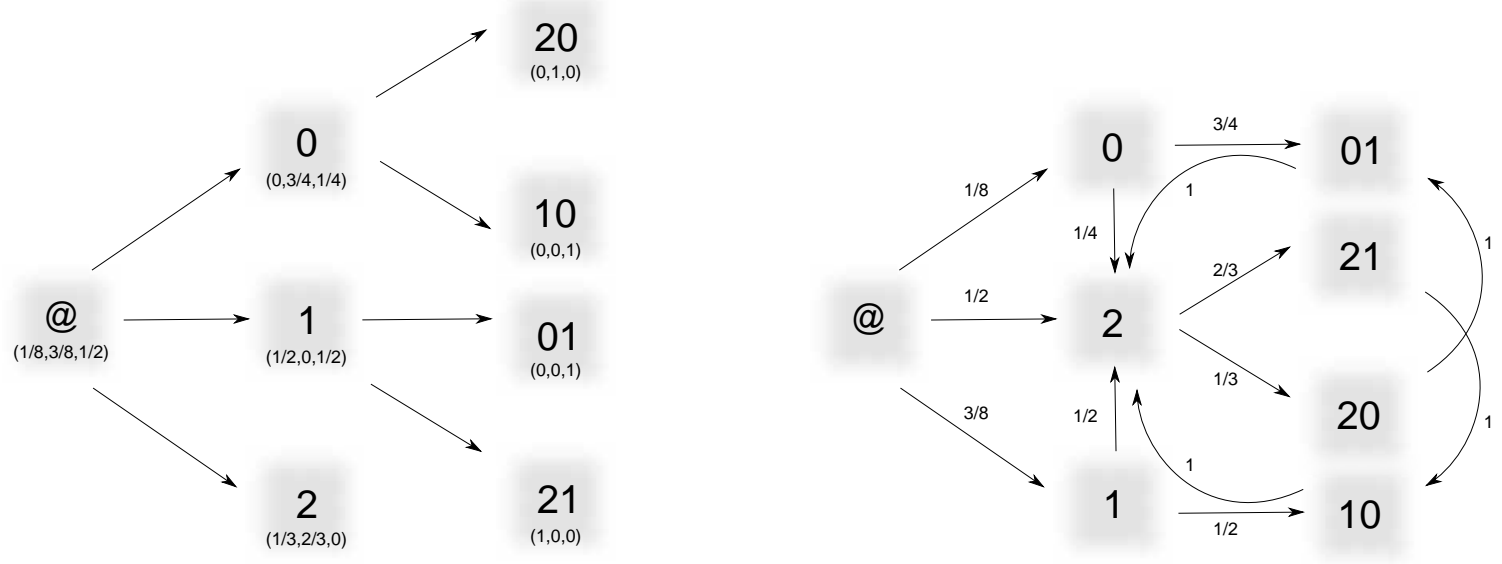

Figure 2: A transformation from a PST to a PSA [14].

\subsection{T-Pattern recognition}

The concept and algorithm behind T-Patterns was first stimulated by research regarding the structure of behavior and interactions with focus on real-time, probabilistic, and functional aspects, as well as hierarchical and syntactic structure, creativity, routines and planning. $[15,10]$ T-Patterns were chosen, because behavior patterns are often hidden in a behavioral stream and exist at different time scales.

The T-Pattern method described in [10] is an approach to find patterns in time series data, where causality between different data can be expected in short time intervals, relative to the observation period. This TPattern algorithm can be applied to sensor data in smart environments. The activity patterns in real sensor data are expected to be hidden in a data stream created by a large number of sensors. The approach can handle noisy, uncertain and incomplete sensor data, as the algorithm makes a probabilistic method available.[16]

The T-Pattern algorithm works with a bottom-up approach. A bottom-up approach means, that first the simplest T-Patterns are searched for and then, level by level, the higher hierarchical T-Patterns are found. These simple T-Patterns are at the fundamental level just simple pairs of sensor events having a statistical significant interval relationship.[15]

The assumption in the T-Pattern approach is a null hypothesis, expecting that each component is independently and randomly distributed over time with its observed average frequency. This null hypothesis is the assumption which all found T-Patterns are tested against.
The significance testing is based on Poisson probability distribution.

Making use of real-time information, the following probabilistic real-time relation serves as a key to the detection of hidden T-Patterns. If $A$ is an earlier and $B$ a later component of the same recurring T-Pattern, then, after an occurrence of $A$ at $t$, there is an interval $\left[t+d_{1}, t+d_{2}\right],\left(d_{2} \geq d_{1} \geq 0\right)$ that tends to contain at least one occurrence of $B$ more often than it would be expected by chance. This relation is called a critical interval (or CI) relation between the distributions of $A$ and $B$. The later component $B$ does not need to be the one immediately following $A$ within the pattern, so even when some elements of a T-Pattern are ignored, it may still have the defining characteristics.

The behavior data are coded during an observation period $\left[1, N_{T}\right]$ in terms of discrete occurrence times of events. A T-Pattern can be seen as an ordered set $Q=X_{1} \approx d t_{1} X_{2} \approx d t_{2} \ldots X_{m}$, where $X$ stand for simpler T-Patterns and each $\approx d t$ term stands for a relatively invariant (relative to the expectation assuming the null hypothesis) time distance between the components, which separates the consecutive $X_{i}$ and $X_{i+1}$ terms.[16] The algorithm as proposed in [10] computes if there is a series of intervals containing $k(k \geq 2)$ occurrences of $A$ followed by at least one $B$ and checks if $k$ is significantly greater than the expectation, by varying the interval length for the search.

The input data are a set of sensor events, where timestamps represent the beginning and ending time of a specific sensor event. This data from real world settings are often sparse during long observational periods 


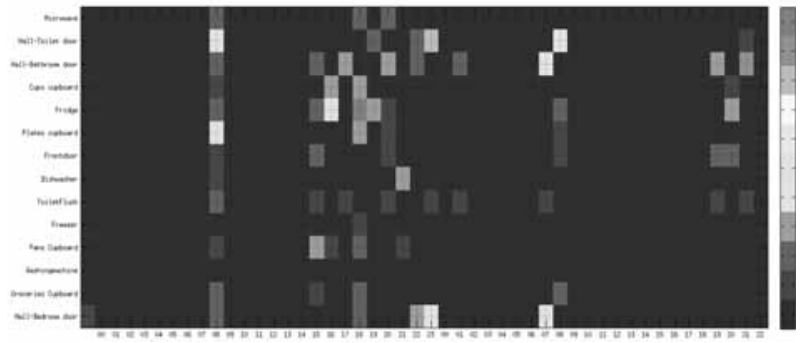

Figure 3: Sensor data recorded 2 days, accumulated hourly.

and clustered within short periods, which can be seen in Figure 3. This figure shows long periods without activity, and short periods with high activity. This problem can be solved by considered the time during the use of the T-Pattern approach. A small significance level leads to more reliable results as not less long patterns will be recognized $\left(\alpha=10^{-8}\right)$.

Some patterns appeared significantly more often than others, and those are essential for an activity to be recognized.

\subsection{Suffix Trees and Probabilistic Finite Automata}

Activity processes can be described by a subclass of probabilistic finite automata which are called Probabilistic Suffix Automata (PSA) by the authors of [4]. Probabilistic automata have similarities to widely used Hidden Markov Models, as analyzed in [17]. On the other hand PSAs seemed to be a logical extension to handle uncertainty in cases which can not be solved with pure finite state automata.

A PST $T$, over an alphabet $\Sigma$, is a tree of degree $|\Sigma|$. Each edge in the tree is labeled by a single symbol in $\Sigma$, such that from every internal node there is exactly one edge labeled by each symbol. The nodes of the tree are labeled by pairs $\left(s, \gamma_{s}\right)$, where $s$ is the string associated with the walk starting from that node and ending in the root of the tree, and $\gamma_{s}: \Sigma \rightarrow[0,1]$ is the next symbol probability function related with $s$. It is required that for every string s labeling a node in the tree, $\Sigma_{\sigma \in \Sigma} \gamma_{s}(\sigma)=1$. A PST $T$ generates strings of infinite length, but only probability distributions induced on finite length prefixes of these strings are considered. The probability that $T$ generates a string $r=r_{1} r_{2} \ldots r_{N} \in \Sigma^{N}$ is

$$
P_{T}^{N}(r)=\prod_{i=1}^{N} \gamma_{s^{i-1}}\left(r_{i}\right)
$$

where $s^{0}=e$, the empty string, and for $1 \leq j \leq N-1, s^{j}$ is the string labeling the deepest node reached by taking the walk corresponding to $r_{i} r_{i-1} \ldots r_{1}$ starting at the root of $T$.

The PST is learned with a top-down algorithm by means of the sample statistic. With this T-Patterns the empirical probability function $\tilde{P}$ is defined and a suffix tree $\bar{T}$, which is a sub-tree of $T$ with high probability, is constructed.

First, only a single node labeled as empty string $e$ is constructed. Further nodes are added which are reasonably to be in the tree. This is the case when for the node $v$, the empirical probability of the suffix $s, \tilde{P}(s)$, is non negligible. Additionally, for $\sigma$ the empiric probability of $s$ followed by $\sigma, \tilde{P}(\sigma \mid s)$, must differ significantly from the probability of observing $\operatorname{suffix}(s)$ followed by $\sigma, \tilde{P}(\sigma \mid \operatorname{suffix}(s))$. Suffix(s) is the suffix string of the parent node, meaning the way from the root to the parent node of $v$.

The decision rule is dependent on the ratio between $\tilde{P}(\sigma \mid s)$ and $\tilde{P}(\sigma \mid s u f f i x(s))$. A given node is only added if this ratio is substantially greater than one. The tree is built level by level so the successors of a leaf are only added if they satisfy the condition. The tree reaches its final shape, if it does not fulfill the condition anymore.[4]

\section{Results}

In this section the results of the methods described so far are shown and discussed. The data from Kasteren $e t$ al. [13] are used to evaluate the T-Pattern algorithm and the results fulfills the demand. The last part focuses on the results from the algorithm constructing a PSA.

\subsection{T-Pattern Analysis}

The T-Patterns are evaluated with the annotated data in Matlab. In this analysis the significance test for the TPatterns is done by the Binomial distribution. For each pattern the best fitting activity is searched for and is saved in the class with the highest percentage of overlap. These percentages are visualized in a pie and a histogram format.

In Figure 4, the histogram bars describes in 10\% steps the percentage of patterns intersecting with an annotated activity. For better understanding 0 means no match with an activity, 1 means all patterns which 


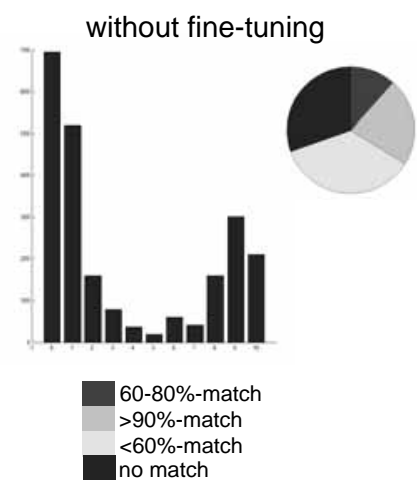

no sensor-event patterns

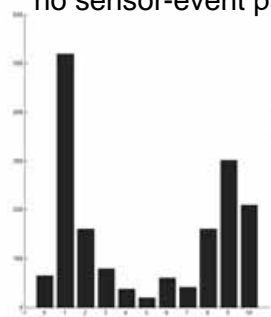

$10 \%$ activity blur

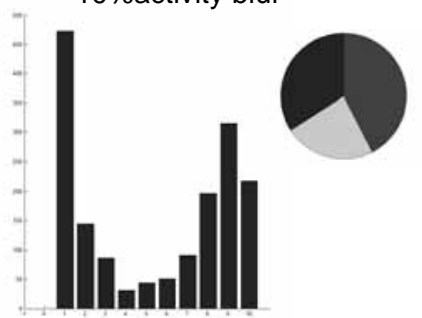

60-80\%-match $>90 \%$-match $<60 \%$-match

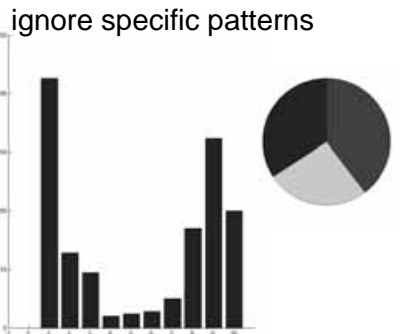

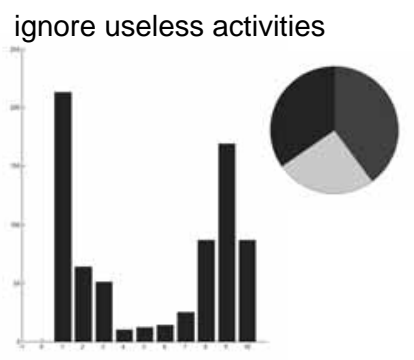

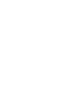

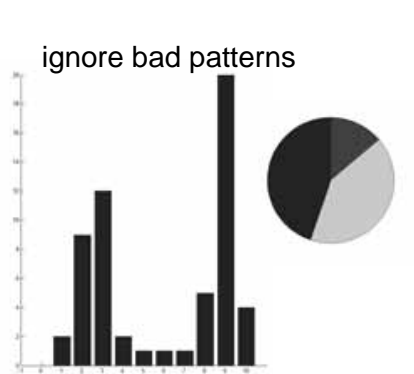

Figure 4: Fine Tuning.

intersect $0-10 \%$ with an annotated activity, 2 means $10-20 \%$ intersection and so on.

The pie has four different classes, 'no matches', 'more than 60\%-matches', 'between $60 \%$ and $80 \%$ matches' and 'more than $90 \%$-matches'. This figures are all analyzed over the whole time period with the TPattern algorithm focusing on the sensor Ids 7 and 8 . Sensor Id 7 stands for the 'toilet flush' and sensor Id 8 for the 'toilet door'. The figures must be seen as a continuous chain. All patterns which are left out in one picture are also left out in the other pictures in vertical direction.

In the first evaluation the T-Pattern algorithm finds too many T-Patterns compare picture one. This is the reason why a fine tuning of the recognized T-Patterns has been done to get more appropriate patterns. Especially the first two bars are a problem for further evaluation. This are those patterns which do not intersect with an activity or only intersect with at most $10 \%$. In the first picture second row the T-Patterns which consist of only one sensor event are left out. A huge improvement can be seen especially in the 'no matches' part, as most of the individual single sensor-events are too small to overlap with an activity.

In the second picture the fine tuning is expanded by blurring the activities $10 \%$ each side. Other fine tuning steps like ignoring specific patterns, useless patterns and bad pattern lead finally to an appropriate result.

The significance analysis leads to the result of an improvement of while using lower significance level. The significance level 0.005 or 0.001 seems to be more appropriate than a bigger one like 0.05 . The significance level is lowered with the consequence of less patterns being recognized. This patterns are those which match an annotated activity with higher percentage.

\subsection{Probabilistic Suffix Tree and Probabilistic Suffix Automata}

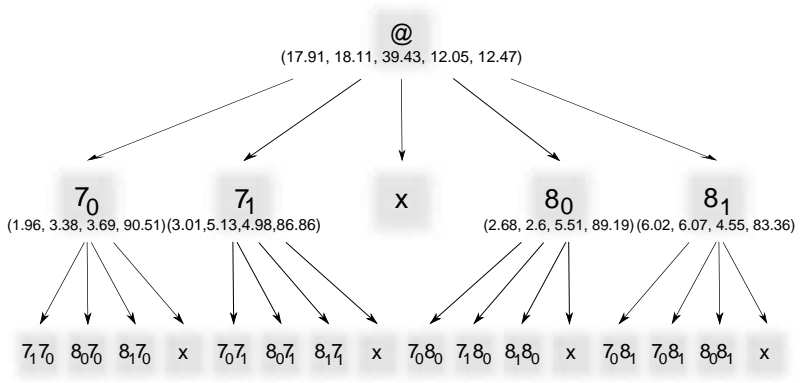

Figure 5: PST based on sensor 7 and 8 with pattern length 2 .

In Figure 5 a result of the used system can be seen. In this case the sensors 7 and 8 are considered to build a PST. The probabilities are described in percentage, 


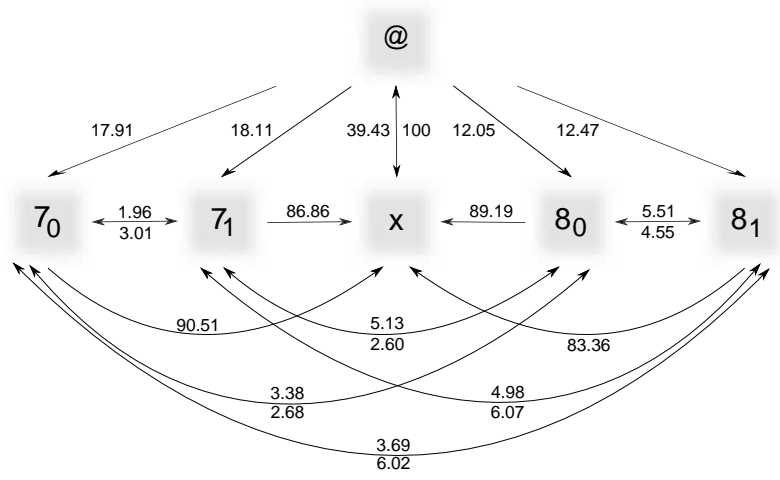

Figure 6: PSA transformed from PST in 5.

where the state $8_{0}$ means 'toilet flush usage ended', 81 'toilet flush usage started', $7_{0}$ 'toilet door contact opened' and $7_{1}$ means 'toilet door contact closed'. The results show that the probabilities are reasonable, as in each step the probabilities get smaller. This is of course true, because the occurrence of event $A$ is at least as probable as the occurrence of pattern $A B$.

In Figure 6 the PST is transformed into a PSA with the already mentioned method. The result is an automaton, because for each state exists a subsequent state. If the activity, for instance 'using the toilet', is finished, the activity concludes with the state $x$, describing all events excluding the important ones 7 and 8 . The two probabilities in Figure 6 assigned to arrows pointing in both directions describe the probability to the left node, indicated by the number above, and to the right node, indicated by the number below.

\section{Conclusion and Outlook}

The work describes a system which is able to deal with sequential data from domestic sensors. This sensor data are used to detect patterns and transform this patterns into knowledge, which can be further used to detect anomalies. This is done with the transformation of the T-Pattern sample statistic to a PST and further to a PSA. This PSA only differs in the arcs and not in the nodes or probabilities from the PST.

The algorithms described beforehand will fundamentally increase the efficiency of a home automation system in terms of configuration efforts due to automated processes. A conceptual structure of the overall system is depicted in Figure 7. The data from the Smart Home System from sensors and actuators are preprocessed, this means false data are deleted. After that the

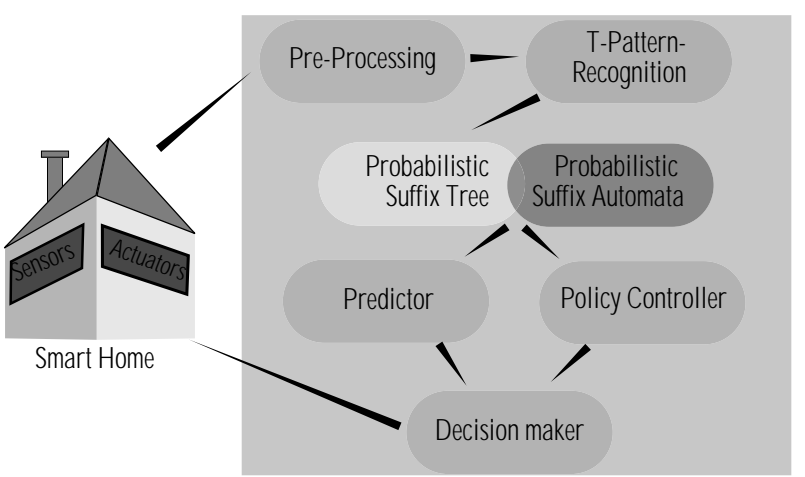

Figure 7: Activity recognition structure.

algorithm from above is applied to get a PSA.

The work ends with in this step, but further research can make it possible to detect anomalies on the basis of this algorithm. The idea behind is to use the prediction data from PSA and from policy controller, which contains user preferences and statistical data, for a decision maker. This decision maker, not yet enveloped, would be able to decide if a specific event occurred like leaving the Smart Home or optionally reminding the user to put on a jacket. The anomalies detection can also give hints about physical health status and diseases.[5]

\section{References}

[1] Nugent CD, Biswas J, Hoey J. Activity Recognition in Pervasive Intelligent Environments (Google eBook). Springer. 2011.

[2] Han JW, Pei J, Yan XF. From sequential pattern mining to structured pattern mining: a pattern-growth approach. Journal of Computer Science and Technology. 2004; 19(3):257-279.

[3] Li K, Fu Y. Prediction of Human Activity by Discovering Temporal Sequence Patterns. IEEE Transactions on Pattern Analysis and Machine Intelligence. 2014;36(8):1644-1657.

[4] Ron D, Singer Y, Tishby N. The Power of Amnesia : Learning Probabilistic Automata with Variable Memory Length. Machine Learning. 1996;25(2):117-149.

[5] Van Den Broek G, Cavallo F, Wehrmann C. AALIANCE Ambient Assisted Living Roadmap. Amsterdam, The Netherlands, The Netherlands: IOS Press. 2010.

[6] Mori T, Fujii A, Shimosaka M, Noguchi H, Sato T. Typical Behavior Patterns Extraction and Anomaly Detection Algorithm Based on Accumulated Home Sensor Data. In: Future Generation Communication and Networking (FGCN 2007). IEEE. 2007; pp. 12-18. 
[7] Albrecht DW, Zukerman I, Nicholson AE. Bayesian Models for Keyhole Plan Recognition in an Adventure Game. In: User Modeling and User-Adapted Interaction, 1. Springer Netherlands. 1998; pp. 5-47.

[8] Unsupervised Learning. In: Advanced Lectures on Machine Learning, edited by Bousquet $\mathrm{O}$, von Luxburg U, Rätsch G, vol. 3176 of Lecture Notes in Computer Science. 2004;.

[9] Paz A. Introduction to Probabilistic Automata. New York: Academic Press. 1971

[10] Magnusson MS. Discovering hidden time patterns in behavior: T-patterns and their detection. Behavior research methods, instruments, \& computers : a journal of the Psychonomic Society, Inc. 2000;32(1):93-110.

[11] T-pattern analysis for the study of temporal structure of animal and human behavior: A comprehensive review. Journal of Neuroscience Methods. 2015;239(0):34 - 46.

[12] Fallmann S, Kropf J. Prediction of sequential patterns of domotic sensor data considering variable temporal pattern length. In: WISHWell14 Proceedings- 6th International Workshop on Intelligent Environments Supporting Healthcare and Well-being. Eindhoven. 2014; .

[13] van Kasteren TL, Englebienne G, Kröse BJA. Human Activity Recognition from Wireless Sensor Network Data: Benchmark and Software. In: Atlantis Ambient and Pervasive Intelligence, edited by Chen L, Nugent CD, Biswas J, Hoey J, vol. 4, pp. 165-186. Amsterdam: Atlantis Press. 2011;

[14] Mazeroff G, De V, Jens C, Michael G, Thomason G. Probabilistic Trees and Automata for Application Behavior Modeling. In: 41st ACM Southeast Regional Conference Proceedings. 2003; pp. 435-440.

[15] Magnusson MS. Repeated Patterns in Behavior and Other Biological Phenomena. In: Evolution of Communication Systems: A Comparative Approach, edited by Oller DK, Griebel U, pp. 111-128. Cambridge, MA: MIT Press. 2004;.

[16] Kropf J, Roedl L, Hochgatterer A. A modular and flexible system for activity recognition and smart home control based on nonobtrusive sensors. In: Pervasive Computing Technologies for Healthcare (PervasiveHealth), 2012 6th International Conference on. IEEE. 2012; pp. 245-251.

[17] Dupont P, Denis F, Esposito Y. Links between probabilistic automata and hidden Markov models: probability distributions, learning models and induction algorithms. Pattern Recognition. 2005; 38(9):1349-1371. 


\title{
Modeling, Simulation and Control of a Loading Bridge
}

\author{
Marko Hančič, Gorazd Karer* , Igor Škrjanc
}

Faculty of Electrical Engineering, University of Ljubljana, Tržaška cesta 25, SI-1000 Ljubljana, Slovenia; *gorazd.karer@fe.uni-lj.si

Simulation Notes Europe SNE 25(3-4), 165 - 170

DOI: $10.11128 /$ sne.25.tn. 10309

Received: November 17, 2015;

Accepted: November 30, 2015

Abstract. This paper is focused on modeling, simulation and validation of the mathematical model of a loading bridge by comparison to the real system. A Lagrangian modeling method is presented to define a mathematical model of the loading-bridge dynamical system. Using the Matlab-Simulink simulation environment we have estimated the parameters and validated the model with regard to the experiments on the real device. The discrepancy between the simulated and the measured responses was adequate. Hence, we can conclude that the developed nonlinear model provides a satisfactory description of real system behaviour. Using the model, we designed a fuzzy controller for controlling load position and load height. The obtained controller has also been tuned and tested on the real device.

\section{Introduction}

In everyday engineering practice we are often faced with the problem of process-control design. When dealing with processes expressing relatively complex dynamic properties that are difficult to control or even dangerous when not taken care of properly, it is generally sensible to first describe the process as a mathematical model so as to be able to carry out the possibly problematic experiments and conduct the first stages of the control-design in a safe simulation environment. In this regard, we deal with a loading-bridge dynamical system. In order to be able to design a suitable controller and, in addition, to test the dynamical properties of the loading bridge, we have described the dynamics of the real device with a mathematical model, developed a simulator of the system and designed a fuzzy controller for the device.

\section{Description of the Loading Bridge}

The loading bridge Amira PS600 (see Figure 1) consists of an aluminium frame covered with sheets of plexiglass. Inside the frame, there is a guiding bar, along which the cart is driven by means of a DC-motor, a clutch, a tooth wheel and a tooth belt. The length of the bar is approximately $1.5 \mathrm{~m}$. Two proximity switches are mounted close to both ends of the guiding bar. They are used for limiting the position of the cart. The position of the cart is measured by an incremental encoder.

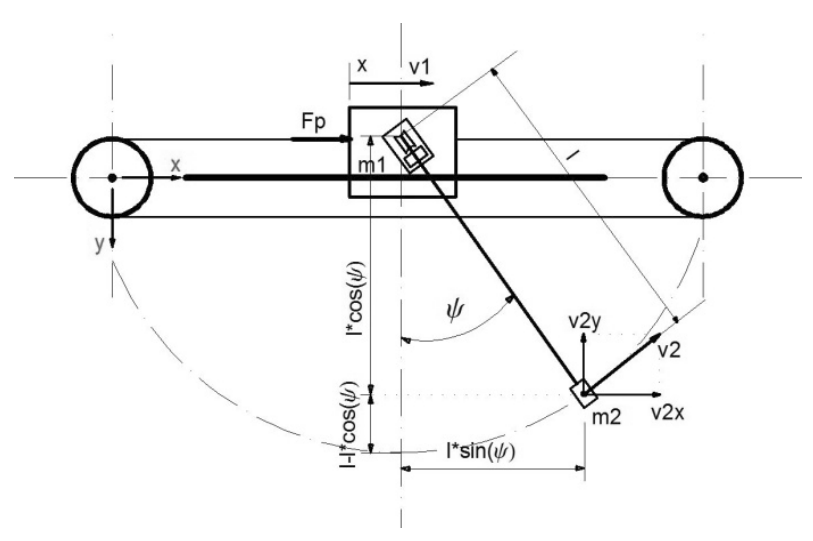

Figure 1: A scheme of the loading bridge with annotated quantities: $F_{p}$ - driving force on the cart, $\psi$ swing angle of the load, $I$ - rope length, $x$ - cart position, $m_{1}$ - cart mass, $m_{2}$ - load mass, $v_{1}$ - cart velocity, $v_{2}$ - load velocity, $v_{2 x}$ - horizontal component of load velocity, $v_{2 y}$ - vertical component of load velocity.

The cart carries a winch that is used to change the length of the rope. The rope runs around two deflection rollers and carries the load, which is suspended below the guiding bar. The length of the rope is measured by an incremental encoder. 
The loading bridge allows the load to swing in parallel to the guiding bar when the cart is moved to the left or to the right. The swing angle is measured by an incremental encoder [3]. Table 1 shows the measured parameters of the loading bridge.

\begin{tabular}{cc}
\hline Parameter & Value \\
\hline $\mathbf{r}$ & $0.3183 \mathrm{~m}$ \\
\hline $\mathbf{m}_{\mathbf{t}}$ & $0.2 \mathrm{~kg}$ \\
\hline $\mathbf{m}_{\mathbf{1}}$ & $5.5 \mathrm{~kg}$ \\
\hline $\mathbf{m}_{\mathbf{2}}$ & $0.143 \mathrm{~kg}$ \\
\hline
\end{tabular}

Table 1: The measured parameters of the loading bridge. Here, $r$ is the radius of the winch, $m t$ is the mass of the winch, $\mathrm{ml}$ denotes the mass of the cart and $\mathrm{m} 2$ the mass of the load.

\section{Derivation of the}

\section{Mathematical Model}

The mathematical model of the loading bridge was derived using the Lagrangian method [1,2]. A general Lagrangian partial differential equation is given in eq. (1).

$$
\frac{d}{d t}\left(\frac{\partial L}{\partial \dot{q}_{s}}\right)-\frac{\partial L}{\partial q_{s}}+\frac{\partial P}{\partial \dot{q}_{s}}=F_{s}
$$

Here, $q_{s}$ is a generalized coordinate describing the model of the system. $P$ represents the power-dissipation function - see eq. (2) - where $R$ is the damping factor.

$$
P=\frac{1}{2} R \dot{q}_{s}^{2}
$$

$L$ stands for the Lagrangian in eq. (3).

$$
L=T_{k}-V_{p}
$$

Here, $T_{k}$ is the kinetic energy of the system and $V_{p}$ represents the potential energy of the system. $F_{S}$ stands for the external excitation along the respective generalized coordinate. By considering the kinetic and potential energy of the system, the Lagrangian can be rewritten as in eq. (4).

$$
\begin{aligned}
L=\frac{1}{2} \dot{x}^{2}\left(m_{1}+m_{2}\right) & +\frac{1}{2} \dot{\psi}^{2} l^{2} m_{2}+\frac{1}{2} \dot{l}^{2} m_{2} \\
& +\dot{x} m_{2} \sin (\psi)+\dot{x} \dot{\psi} l m_{2} \cos (\psi) \\
& +\frac{1}{2} J\left(\frac{\dot{i}^{2}}{r^{2}}\right)+m_{2} g l \cos (\psi)
\end{aligned}
$$

Here, $J$ is the moment of inertia of the winch, $r$ is its radius and $g$ represents gravitational acceleration.

Based on eq. (1) a partial differential equation for each of the generalized coordinates can be established. In our case, there are 3 generalized coordinates, i.e.: cart position $x$ in eq. (5); swing angle $\psi$ in eq. (6); and rope length $l$ in eq. (7):

$$
\begin{aligned}
& \frac{\partial}{\partial t}\left(\frac{\partial L}{\partial \dot{\mathbf{x}}}\right)+\frac{\partial L}{\partial x}+\frac{\partial P}{\partial \dot{\mathbf{x}}}=F_{p} \\
& \frac{\partial}{\partial t}\left(\frac{\partial L}{\partial \dot{\psi}}\right)+\frac{\partial L}{\partial \psi}+\frac{\partial P}{\partial \dot{\psi}}=0 \\
& \frac{\partial}{\partial t}\left(\frac{\partial L}{\partial \dot{\mathrm{l}}}\right)+\frac{\partial L}{\partial \mathrm{l}}+\frac{\partial P}{\partial \dot{\mathrm{l}}}=T
\end{aligned}
$$

Upon inserting the Lagrangian and the powerdissipation function into eqs. (5), (6) and (7) and carrying out the derivations, we obtain the following $2^{\text {nd }}$ order differential equations for $x, \psi$ and $l$ in eqs. (8), (9) and (10), respectively.

$$
\begin{aligned}
& \ddot{x}=\left(m_{1}+m_{2}\right)^{-1}\left[F_{p}-R_{t r} \dot{x}-\ddot{l} m_{2} \sin (\psi)\right. \\
& -2 \dot{\psi} l \dot{m}_{2} \cos (\psi)-\ddot{\psi} l m_{2} \cos (\psi)(8) \\
& \left.+\dot{\psi}^{2} \operatorname{lm}_{2} \sin (\psi)\right] \\
& \ddot{\psi}=l^{-1}\left[-\ddot{x} \cos (\psi)-g \sin (\psi)-2 \dot{\psi} \dot{l}-R_{p s i} * \dot{\psi}\right] \\
& \ddot{l}=\left(m_{2}+\frac{J}{r^{2}}\right)^{-1}\left[T-R_{t r l} \dot{l}-\ddot{x} m_{2} \sin (\psi)\right. \\
& \left.+\dot{\psi}^{2} l m_{2}+m_{2} g \cos (\psi)\right]
\end{aligned}
$$

The aforementioned equations define the mathematical representation of the system.

\section{Simulation and Validation of the Mathematical Model}

The nonlinear mathematical model of the loading bridge was implemented in the Matlab/Simulink simulation environment. The simulation scheme of the nonlinear model is presented in Figure 2. 


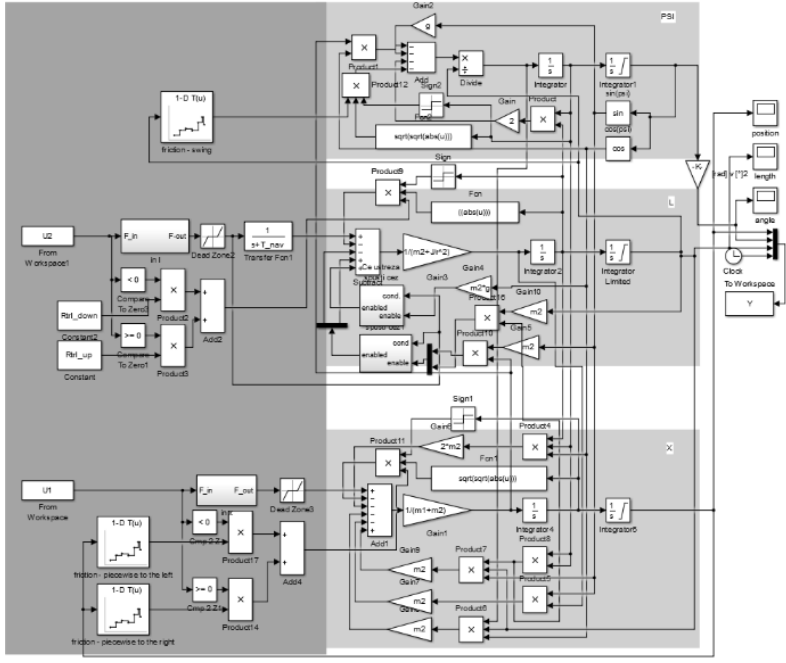

Figure 2: Simulation scheme of the nonlinear model of the loading bridge.

In order to validate the obtained nonlinear model, the responses of the model were compared to the real loading-bridge response-signal measurements using the scheme in Figure 3.

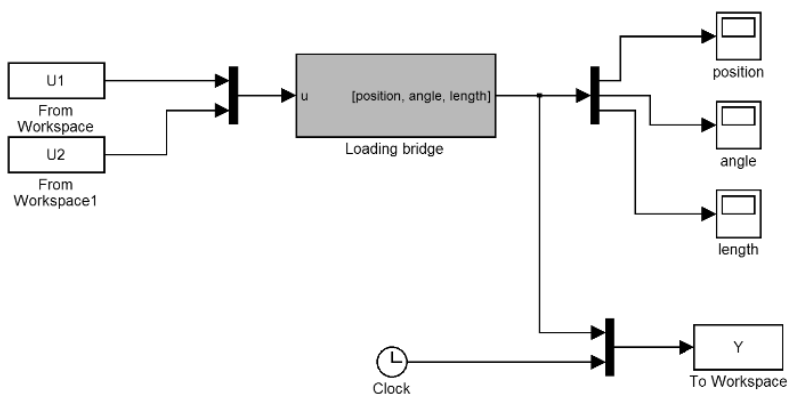

Figure 3: Measurement scheme for acquiring real loadingbridge response signals.

\subsection{Parameters of the nonlinear model}

The real loading bridge and the developed nonlinear model were fed a positive and negative step-signal for the cart-motor input and the appropriate response signals were recorded (see Figure 4 - left). Figure 4 depicts the comparison of signals of the nonlinear model and the measured signals on the real plant. We can see that the cart does not return to the initial position (see Figure 4 - right).
The dynamical model had to be adjusted in order to close the discrepancy gap between the model and the real device response signals. The friction parameters are not constant along the guiding bar. In addition, they depend on the direction the cart is moving as well. Therefore, a series of measurements was conducted, taking into account the relevant partitions of the guiding bar. A testing input signal was used that moved the cart to the left and to the right. Descending and ascending ramps were used for the measurements, respectively. The respective ramps were time-limited and amplitudelimited.

The distance travelled was used to estimate the friction parameters appurtenant to the relevant partition of the guiding bar. In such a manner, the developed model includes several lookup tables that describe the nonlinear dynamics within a particular partition. The main cause for nonlinearity is non-constant friction along the guiding bar and a dead-zone of the input signal that provides the voltage to the motor.

In order to estimate the damping properties of the pendulum, which is made up of the load and the loadcarrying rope, several responses to the initial nonequilibrium condition were measured. The measurements were initially carried out without moving the cart along the guiding bar. The responses were measured with various lengths of the rope $l$. The damping coefficient was adjusted until the response of the nonlinear model matched the response of the real device.
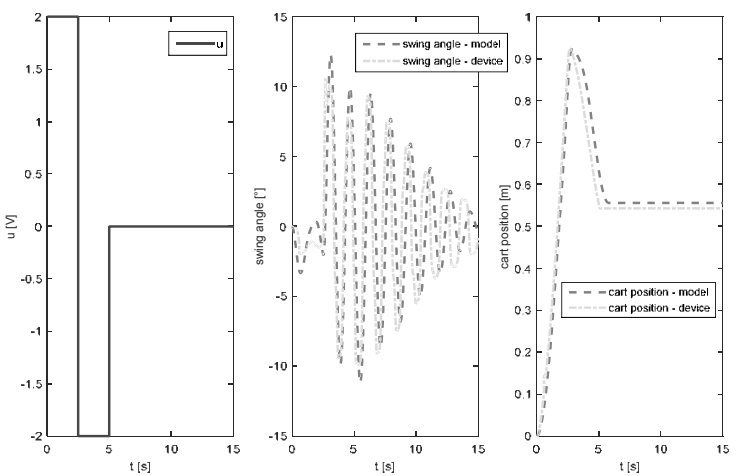

Figure 4: Validation input signal (positive and negative steps: $2 \mathrm{~V}$ and $-2 \mathrm{~V}$, respectively) for the cart and response comparison between the nonlinear model (blue line) and the real device (red line). The rope length was constant $(I=0.7 \mathrm{~m})$. 
The responses regarding the change of the rope length as a consequence of the input signal of the winch were also adjusted. In order to match the response of the model to the response of the real device, a dead-zone within the input-voltage has to be considered. Furthermore, the winch submodel includes a $1^{\text {st }}$ order delay. The measurements suggest that the discrepancy between both response signals is acceptable.

\section{Tracking Control of the Load Along the Reference Trajectory}

Tracking control of the load along the reference trajectory was designed by implementing two fuzzy controllers. One was used to control the position of the cart and the other one controlled the length of the rope that carries the load. The reference trajectory is automatically generated from the initial and the final position within Matlab.

The fuzzy controller for the cart position has 3 input variables and 1 output variable, whereas the fuzzy controller for the rope length has 2 input variables and 1 output variable. Both are Mamdani type controllers with centroid defuzzyfication. Table 2 shows the input and output variables and the respective ranges for both fuzzy controllers.

\begin{tabular}{ccccc}
\hline \multirow{2}{*}{ Controller } & Variable & $\begin{array}{c}\text { Lower } \\
\text { Limit }\end{array}$ & $\begin{array}{c}\text { Upper } \\
\text { Limit }\end{array}$ & Unit \\
\hline \multirow{4}{*}{$\begin{array}{c}\text { Cart } \\
\text { position - } x\end{array}$} & Error $\mathrm{x}_{\mathrm{r}}-\mathrm{x}$ & -3 & 3 & {$[\mathrm{~m}]$} \\
\cline { 2 - 5 } & Velocity $\dot{x}$ & $-0,7$ & 0,7 & {$[\mathrm{~m} / \mathrm{s}]$} \\
\cline { 2 - 5 } & Swing angle $\Psi$ & -45 & 45 & {$\left[{ }^{\circ}\right]$} \\
\cline { 2 - 5 } & Motor voltage & -3 & 3 & {$[\mathrm{~V}]$} \\
\hline \multirow{3}{*}{ Rope length - I } & Error $\mathrm{I}_{\mathrm{r}}-\mathrm{I}$ & -3 & 3 & {$[\mathrm{~m}]$} \\
\cline { 2 - 5 } & Velocity $i$ & $-0,7$ & 0,7 & {$[\mathrm{~m} / \mathrm{s}]$} \\
\cline { 2 - 5 } & Motor voltage & -10 & 10 & {$[\mathrm{~V}]$} \\
\hline
\end{tabular}

Table 2: Input and output variables and their respective ranges for both fuzzy controllers.

In both cases, the errors are normalized to the interval $[-1,1]$, so that the controller is comparably responsive within the operating range.
Figure 5 depicts the membership function of the fuzzy controller for cart position, whereas Figure 6 shows the membership function of the fuzzy controller for rope length. The membership functions regarding the error in both cases are condensed around 0 , which ensures high accuracy of control.

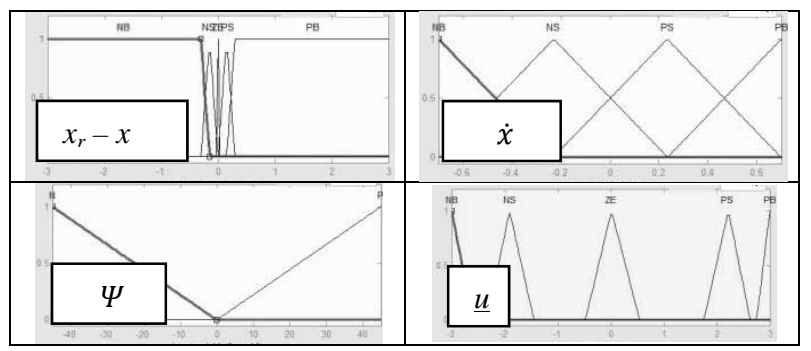

Figure 5: Membership functions of the input and output variables of the fuzzy controller for cart position. Trapezoidal, triangular and Gaussian membership functions are used.

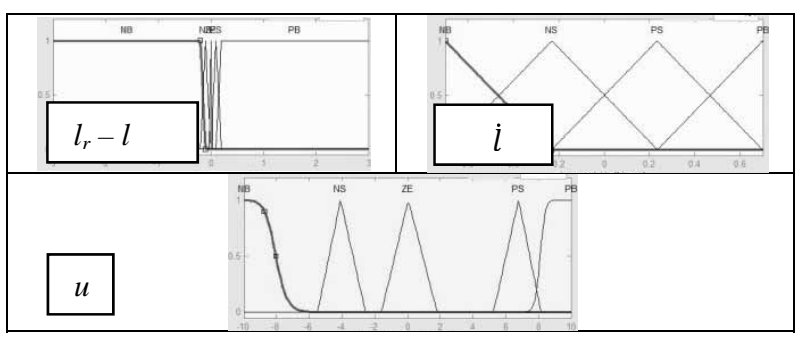

Figure 6: Membership functions of the input and output variables of the fuzzy controller for rope length. Trapezoidal, triangular and Gaussian membership functions are used.

The inputs and outputs of each fuzzy controller are connected by an inference system, which are defined by appropriate rules, as defined below:

1. If (Error is $N B$ ) then (Voltage is $N B$ ).

2. If (Error is $P B$ ) then (Voltage is $P B$ ).

3. If (Error is NS) then (Voltage is NS).

4. If (Error is PS) then (Voltage is PS).

5. If (Error is NS) and (Velocity is PB) then (Voltage is NS).

6. If (Error is PS) and (Velocity is NB) then (Voltage is $P S)$.

7. If (Error is ZE) then (Voltage is ZE).

8. If (Angle is $N$ ) then (Voltage is ZE).

9. If (Angle is $P$ ) then (Voltage is $Z E$ ). 
Both controllers employ similar rules, except for rules 8 and 9, which are not considered in the controller for rope length. On one hand, rules from 1 to 7 tend to minimize the error by appropriately adjusting the motor voltage. On the other hand, rules 8 and 9 are used to slow down the cart to prevent the swing angle of the load exceeding safe boundaries.

Figure 7 shows the control scheme where both fuzzy controllers are implemented. The reference trajectory was generated from the initial position of the load $T_{z}=[0,0.185]$ along the first reference goal-point $T_{1}=[1.2,0.6]$ and ending up in the final reference goalpoint $T_{2}=[0.5,0.3]$.

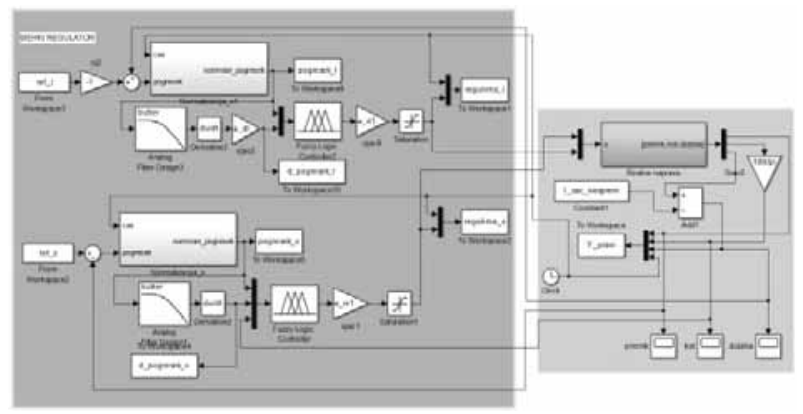

Figure 7: Control scheme with two fuzzy controllers. The same controllers are implemented in the simulation experiment using the nonlinear model and in the experiment using the real device.

The experiments are carried out by first moving the load from the initial position $T_{z}$ to the first reference goalpoint $T_{l}$ and then moving the load to the final reference goal-point $T_{2}$. Figure 8 depicts the comparison between the responses of the model (red line) and of the real device (green line), with regard to the reference trajectory (blue line). The measurements suggest that the discrepancy between the responses is quite small.
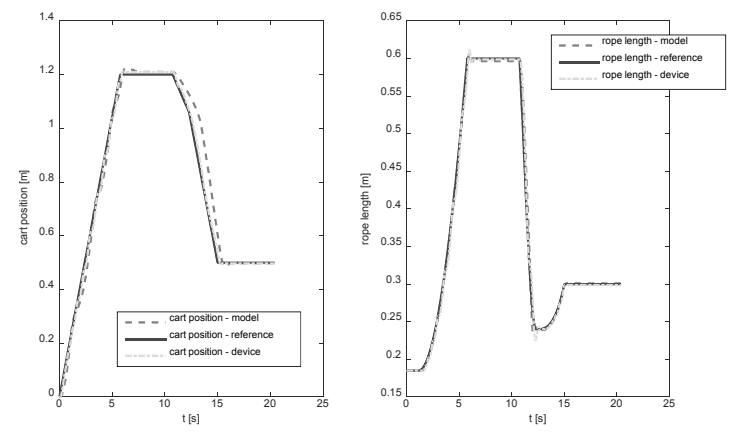

Figure 8: Comparison between the responses of the model (red line) and of the real device (green line), with regard to the reference trajectory (blue line).
Figure 9 shows the control signals for the cart position (left) and for the rope length (right). Red line denotes the signals of the nonlinear model, whereas green line is for the signals concerning the real device.
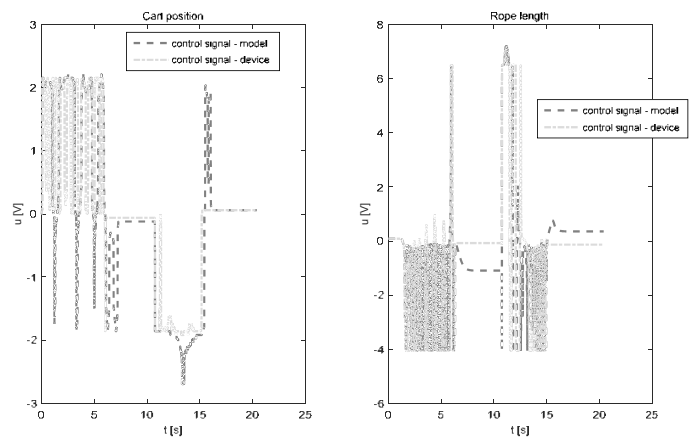

Figure 9: Control signals for cart position (left) and for rope length (right). Red line - nonlinear model; green line - real device.

The behaviour of the simulated model and of the real device are rather similar, although the actuators of the real device are slightly more loaded. Reference tracking of the cart position is favourable both in simulation and on the real device, despite noticing slight overshoots and a negligible steady-state error. Reference tracking o the rope length is also satisfactory in both cases, in spite of minor overshoots. Figure 10 depicts the trajectory of the load from $T_{z}=[0,0.185]$ along $T_{1}=[1.2,0.6]$ to $T_{2}=[0.5,0.3]$.

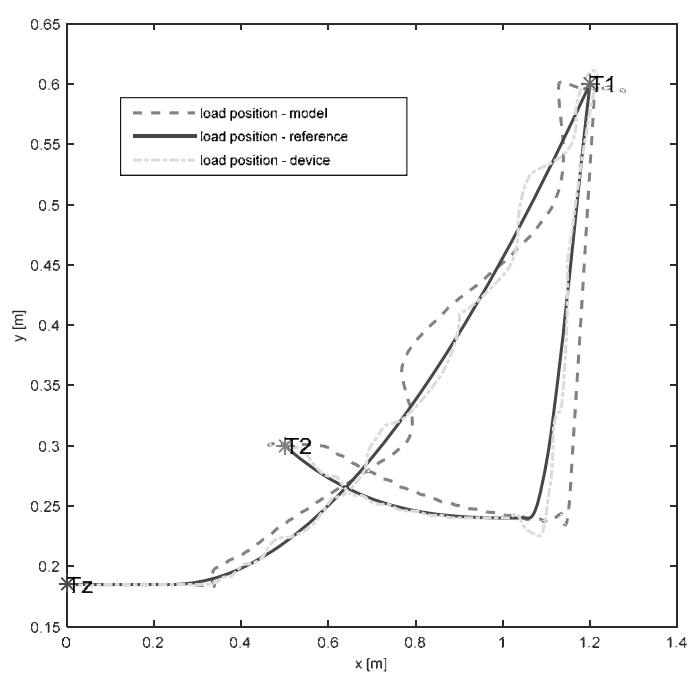

Figure 10: Trajectory of the load: reference (blue line), simulated model response (red line), real device response (green line). $T_{z}=[0,0.185]$ - initial position; $T_{1}=[1.2,0.6]$ - first reference goal-point; $T_{2}=[0.5,0.3]-$ final reference goal-point. 


\section{Conclusion}

The paper illustrates the development of the mathematical model of the loading bridge using the Lagrangian modeling method. The first iteration of the developed model does not fully encompass the dynamics of the real device due to nonmodeled phenomena and repeatability issues. Therefore, the model has been enhanced in order to close the gap between its simulated responses and the responses of the real device.

Two fuzzy controllers - i.e., one for the cart position and ono for the rope length - have been designed and tested. The structure of both controllers is similar, although two additional rules are implemented in the controller for cart position so as to limit the swing angle of the suspended load. Simulation experiments and experiments with the real device lead to conclusion that good control performance can be achieved using the designed fuzzy controllers when tracking the reference trajectory of the load position. Furthermore, the developed model provides the basis for a safe and convenient environment for testing various control strategies. Successful experiments assure that the developed controles can be adequately implemented on the real loading bridge.

\section{References}

[1] Atanasijevič Kunc M. Metode modeliranja-Zapiski predavanj, Faculty of Electrical Engineering, University of Ljubljana, 2013.

[2] Atanasijevič Kunc M. Modeliranje procesov: zbirka primerov z ilustracijami v okolju Matlab-Simulink, Faculty of Electrical Engineering, University of Ljubljana, 2008.

[3] Amira PS600 V2.0; Laboratory Experiment Loading Bridge. Amira GmbH, Duisburg, 2000.

[4] Karba R. Modeliranje procesov, Založba FE in FRI, University of Ljubljana, 1999.

[5] Zupančič B, Karba R., Matko D., Škrjanc I. Simulacija dinamičnih sistemov, Ljubljana, Založba FE in FRI, 2010. 


\title{
Cloud Computing Applied to Calibration of Flood Simulation Models
}

\author{
Marco Brettschneider ${ }^{1 *}$, Bernd Pfützner ${ }^{2}$, Frank Fuchs-Kittowski ${ }^{1}$ \\ ${ }^{1}$ Hochschule für Technik und Wirtschaft HTW Berlin; *Marco.Brettschneider@htw-berlin.de \\ ${ }^{2}$ Büro für Angewandte Hydrologie BAH, Berlin
}

Simulation Notes Europe SNE 25(3-4), 2015, 171 - 177

DOI: $10.11128 /$ sne.25.tn. 10311

Received: January 20, 2015 (Selected ASIM SST 2014

Postconf. Publ.); Accepted: July 14, 2015;

\begin{abstract}
Flood water simulations have to be accurate in order to prevent losses and must therefore be based on well calibrated rainfall runoff models of sufficient complexity. laaS cloud computing has be proven useful as a fast and cost-efficient extension of local computers, in the case, using the calibration of the hydrologic modeling system ArCEGMO as an example. On the other hand, cloud-based systems cannot serve as a replacement for a local computer, as long-term usage is still too expensive and there surely are performance differences regarding the offered architectures. Ultimately, the usage of cloud computing as a viable extension of local computing capacities is only reasonable if state of the art computer technologies with a fair pricing sytem can be offered by laaS providers.
\end{abstract}

\section{Introduction}

Flood waters and flash floods are an ever-leading cause for significant destruction in the areas surrounding rivers [1]. In Germany, for example, the Elbe floods of 2002 and 2013 caused damages totaling millions of euros [2]. The simulation of flood waters, used to determine design parameters for the planning of flood protection measures, also serves to aid in predicting a flood event. Thisis of high importance in the preemptive assessment of possible risks from flood waves, allowing ample timeto implement measures for prevention [3]. The simulation itself can be subdivided into one hydrological part to express the runoff processes in the area and another hydraulic part to illustrate the actual flood characteristics. In urban areas the contribution of urban drainage is often added as well [4].
This study focuses on the hydrological aspect of flood water simulation, which is used for general rainfall runoff models.

Depending on the chosen model complexity, the calibration of a rainfall runoff model can be a very timeconsuming process. Robust values for the model parameters can often only be determined by a large number of model runs. Additionally, high-resolution models found in large-scale applications risk reaching the limits of computing capacity rather quickly. If one would like to test several parameterizations, he would first have to think about alternative methodsof computation.

The computing technologies available are constantly changing, and there are now various ways to involve greater computing capacity for a more detailed description of hydrological processes. These new technologies include multi-core and many-core architectures of current processors and graphics chips [e.g. 5], and nonlocal computational resources, which can enhance the local ones. Among the latter, cloud computing is one of the most prominent of these technologies.

Here, the modeler outsources his calculations onto virtual computers which can be generated on demand from server farms of cloud service providers.

The particular potential of the cloud lies in the scaling of resources: for every application, a corresponding configuration of computing power, memory, disk space, and operating system can be found, enabling the potential coupling of multiple virtual machines.

Cloud computing is still in an early stage. Reliable findings on the use of cloud computing in the field of hydrology and, in particular, for the simualtion of flood events are not yet available. There are few reports on the application of cloud computing in hydrology, whereas their focus is mainly on other aspects such as uncertainty analyses, by using precalibrated models [e.g. 6]. 
Because cloud projects can be implemented quickly and at comparatively low monetary costs, it is reasonable to gain first-hand experience in small pilot projects [7]. In this paper, the outsourcing of model calculations for calibrating a rainfall runoff model for flood simulation on virtual machines in the cloud will be discussed. Within this study, particular emphasis is placed on the attainable computing power and the scalability of resources. A company that creates and maintains the models is also always trying to balance the monetary costs with the resulting benefit, which is why the cost aspect is considered here as well.

The paper is organized as follows: in Chapter 2, the hydrological modeling system ArcEGMO is presented as a basis for flood simulations. Also, the potential of cloud computing is briefly discussed, which is then used to frame the requirements for using clouds in the context of flood simulation. Chapter 3 presents the configurations and the setup of an investigation on cloud services based on an example scenario. In Chapter 4, the results of the application example are presented, thereby demonstrating how cloud computing can meet the requirements set in Chapter 2. In Chapter 5, the potential of cloud computing for complex hydrological model calibration is finally evaluated in terms of computing power and monetary cost efficiency, accompanied by an outlook on further developments.

\section{Requirements for Cloud Computing to Assist Rainfall Runoff Model Calibration}

\subsection{Rainfall runoff modeling with the hydrological modeling system ArcEGMO}

The hydrological modeling system ArcEGMO can be applied for physically sound simulations of all relevant processes of the water balance and flow regime in catchments of different forms and scale ranges in space and time $[8,9]$. Its modular design, the possibilities for a variable surface structure and a set of interfaces for coupling with other models allow for the processing of a large variety of practical and scientific problems (Figure 1).
ArcEGMO has been used successfully in a variety of studies on flood generation for different landscape types and field sizes [e.g. 10, 11]. It became clear that the parameterization of the model is crucial for the realistic representation of catchment processes and dynamics. Since the calibration of the model based on real measured values is an iterative process, a large number of model runs is needed to assess the effect of different parameter values on the ability to correctly predict river runoff. With complex models, the computation time can quickly become a limiting factor in the search for optimal parameters. Therefore, in the following it should be evaluated to what extent cloud computing can be specifically helpful as a way of fast acquisition of additional computing capacity to expand locally limited resources.

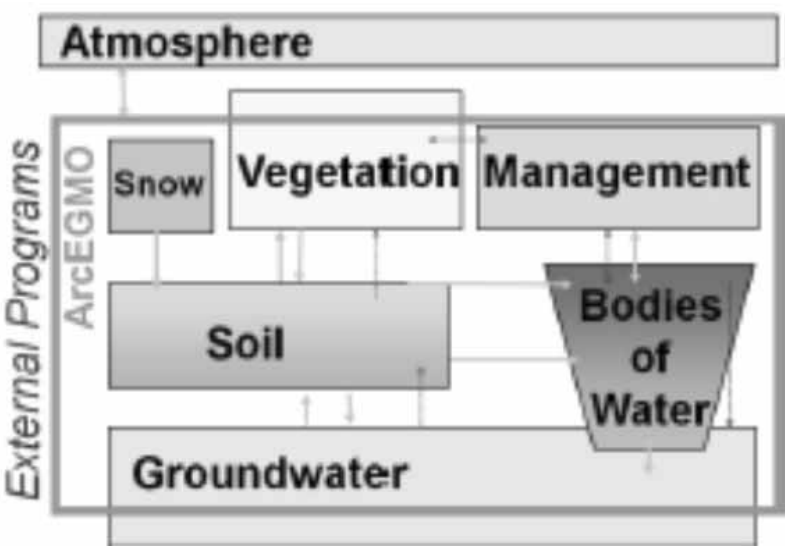

Figure 1. Domains of ArCEGMO.

\subsection{Potential of Cloud Computing}

Cloud computing is a form of flexible and reliable deployment and use of IT resources and IT services. This may be IT infrastructure (network, servers, storage), platforms or applications of all kinds. These are provided as a service over the internet with minimal administrative effort in real time and charged according to consumption [7]. Cloud services are generally subdivided in the three levels of 'Infrastructure as a Service (IaaS)', 'Platform as a Service (PaaS)' and 'Software as a Service (SaaS)'. IaaS provides a minimum infrastructure for computing, used for storing data and network connectivity. The separation from PaaS is blurred, but the latter is usually associated with the development and deployment of Web services. 
With SaaS, complete software is directly provided for online use [12]. Due to the focus on pure processing power IaaS is used in this study and briefly described below.

With IaaS, IT resources are provided as virtual instances with complete virtual hardware and an operating system. From the user's perspective, IaaS looks like actual physical hardware: the user can control the entire operating system and the software running on it, and there are no restrictions in terms of applications that can be hosted by the system. To manage the infrastructure, specific web services are made available, which a user can use to start as many instances (virtual servers) as needed for a particular task in just a few minutes, as well as remove them quickly. The disadvantages of IaaS include the fact that an automatic scaling of the computing entities can be difficult to implement, the cost can increase rapidly if used for an extended period of time, and that there is at least the theoretical possibility of an instance failure [13] .

Companies invest in cloud computing especially because of its potential for cost reduction in situations where extended compute resources are needed only from time to time. Further benefits are in the shift from fixed costs (investments, etc.) to variable costs, as seen in the increase of the flexibility and scalability of IT resources as well as in the demandbased billing of IT services [7].

\subsection{Requirements for the calibration of ArcEGMO on laaS clouds}

A well-calibrated rainfall runoff model is the key ingredient for reliable flood simulations and predictions for preventing damage. The effort required to run several model for an iterative search of robust parameter values can be lowered by cloud computing,but only when the calculation times are the same or shorter as for local computers and the resulting monetary costs remain low. For payment models without longterm booking, the costs depend partly on the time between generating and removing an instance, as well as on additional fees for data storage, data backup and communication with or between instances [14, 15]. The additional services listed above were not considered in this study, because the size, and with that the monetary costs of transferred input and output, are negligible, as is the loss of information and the expected additional costs due to sudden failure of an instance regarding model calibration.
In this survey, the computing times are the determining criterion for assessing the benefits of cloud computing for the calibration of rainfall runoff models. The time consumed by delays in data transfer is important as well, but is usually small compared to computation time, especially when calibrating with lots of spatial elements.

The cloud services of Amazon and Microsoft offer configurations which are comparable to those of regular desktop computers, but also instance types, which are on the same level as modern server computers [16, 17, 18]. The services of Amazon EC2 and Microsoft Windows Azure are therefore used for the upcoming considerations. In addition to the computation times and the monetary costs, the extent to which computing power can be increased by each provider will also be investigated, as well as how the costs evolve in those cases.

\section{Configuration of laas Services for the Calibration of Flood Simulations}

\subsection{Example scenario}

To oppose monetary costs and benefits of cloud computing, many different approaches are already available which focus on the profitability at the scale of larger companies and governmental institutions. These are, for example, based on heuristic methods or break-even analyses [e.g. 15, 19, 20]. Here, the chosen method is also heuristic, but the focus is on the performance of the various types of instances. In the following, we will show based on a realistic scenario, how powerful virtual IaaS machines are in terms of rainfall runoff model calculations with ArcEGMO, with regard to simultaneous consideration of the respective costs. The description of the scenario in terms of the configuration of the model and the IaaS services are the topics of this chapter.

\subsection{Setup of the modeling system ArcEGMO}

ArcEGMO is applied to a real world example catchment, which, in general, is also used for training purposes [9]. The catchment comprises an area of $59 \mathrm{~km}^{2}$ with lowland characteristics and consists of 362 spatial elements (hydrotropes), whereas a period of 5.5 years in daily resolution is calculated. 
The model input data consists of different hydrological and meteorological time series for runoff, precipitation and climate variables. The runoff data is used as the target during model calibration.

After ArcEGMO has been prepared based on its control files, a simulation can be started. The initialization stage of a model run is dominated by file reading and writing processes on the hard drive, while the simulation stage is characterized by memory access. For this reason, a subdivision of the overall calculation time regarding these two stages is applied.

ArcEGMO works sequentially, that is, only one processor core per simulation is used. The amount of memory in use is governed by the number of spatial elements that have to be processed during simulation. In this example scenario, the number is comparatively small, and, therefore, the computation time is short. Because of the sequential processing of ArcEGMO, only scalability based on the different processor types and disk architectures can be considered here. Since currently only Amazon provides multiple computing and storage architectures, the investigation on scalability refers only to EC2.

\subsection{The applied laaS services}

Windows Azure possesses properties both of PaaS and IaaS, and thus covers a large range of possibilities for the development and operation of its own software, with a focus on web applications. Amazon's Web Services (AWS) concentrate on flexibility and the combination of resources provided for any kind of use with focus on IaaS. Moreover, there is a wide range of operating systems which, if chosen, already have additional software and software development tools preinstalled.

Within AWS, EC2 is the service to deploy virtual computing resources. Billing is exact to the hour, additional hard drive and communication capacities can be booked with surcharge [16]. In Windows Azure, virtual machines are started and monitored via the Azure user interface. The selected configuration of the instance and the use of additional services or storage capacities determine again the final costs per hour [18].

\subsection{The Selection of instance configurations}

For both considered cloud providers, configurations have been selected which correspond to the hardware of a local desktop computer.
An exact match and comparison between all three configurations is not possible since both providers use different hardware vendors (Intel for Amazon, AMD for Microsoft). The comparison can therefore be performed only in an approximate way.

The local computer has an E7400 dual-core processor from Intel with 64bit architecture and $2.8 \mathrm{GHz}$ per core, 3 GB RAM, and HDD hard drive. The Amazon m1.large configuration was chosen as the appropriate instance, with 2 cores of an Intel Xeon processor (the clock frequency has not been specified by Amazon), and 7.5 GB RAM [17]. For Windows Azure the A2 configuration with AMD Opteron 2377 EE processor (2 $\mathrm{x} 1.6 \mathrm{GHz}$ cores) and a total of $3.5 \mathrm{~GB}$ of memory has been selected $[18,21]$. Table 1 summarizes all configurations.

\begin{tabular}{lll}
\hline $\begin{array}{lll}\text { Amazon EC2 } \\
\text { m1.large }\end{array}$ & Windows Azure A2 & Local Computer \\
\hline - Intel Xeon Pro- & - AM D Opteron Pro- & - Intel E7400 \\
cessor, 2 Cores, & cessor, 2 Cores à 1,6 & Dual-Core à 2,8 \\
64bit & GHz, 64bit & GHz, 64bit \\
- 7 GB RAM & - 3,5 GB RAM & - 3 GB RAM \\
- Windows Server & - Windows Server & - Windows 7 \\
\hline
\end{tabular}

Table 1. Hardware configuration of the local desktop computer and the selected cloud instance types.

Table 2 shows a summary of both providers for the pricing models that do not require a reservation or subscriptions. The costs are exact to the hour. The costs per 30 days are related to a continuously active instance. Additional fees can be incurred for additional services such as storage, databases, network infrastructure, etc., but these services were not needed here, so their cost has been excluded. A local machine was not listed in this table, since variable and fixed costs must be considered together, which prevents an exact comparison.

\begin{tabular}{lll}
\hline & $\begin{array}{l}\text { Amazon EC2 } \\
\text { m1.large on demand }\end{array}$ & $\begin{array}{l}\text { Windows Azure A2 } \\
\text { pay-as-you-go }\end{array}$ \\
\hline $\begin{array}{l}\text { Price [Euros per } \\
\text { hour] }\end{array}$ & 0.26 & 0.135 \\
\hline $\begin{array}{l}\text { Price [Euros per } \\
30 \text { days] }\end{array}$ & 190.66 & 97.20 \\
\hline
\end{tabular}

Table 2. Costs for cloud computing on Amazon's EC2 and Microsoft's Windows Azure for the pricing models 'on demand' and 'pay-as-you-go' (source: 16, 18). 
In this study, only the pricing models 'on demand' and 'pay-as-you-go' have been investigated. These relate to a spontaneous provision of instances without having them reserved in advance.

To investigate the potential performance increase with improved cloud resources, instances furnished with the latest processor generation were included into the survey. The $\mathrm{m} 3$ instances use Intel Xeon E5- 2670 Sandy Bridge processors and provide SSD hard drives, as well as c3 instances, which, in turn, are equipped with Xeon E5-2680v2 - Ivy Bridge processors [17]. For both the $\mathrm{m} 3$ and $\mathrm{c} 3$ instance types architectures with two cores have been acquired.

On Windows Azure, there currently is only one generation of instances available, which is why the used processors and hard disk capacity should be on the same level of performance. Additional test confirmed this assumption, but the according results are not presented here.

\section{Results of the Investigation of laas Services for the Calibration of Flood Simulations}

\subsection{Comparison of all configurations}

For Microsoft and Amazon, hardware configurations can be found which are similar to those of the local computer, even though a direct comparison is not possible due to differences regarding the hardware component suppliers for both computing services. Furthermore, Amazon also offers the latest processor generation at the level of current server computers. Regarding the long term monetary costs, the acquisition of a local device is cheaper: currently, a computer as it is used and presented here is available for approximately $600 €$ [22].

Also, if other variable costs like e.g. electricity are taken into account as well, IaaS services with costs of around $200 €$ and $100 €$ for a month of demand to pay at Amazon and Microsoft, respectively, are of benefit only in cases of short and shortterm application (Table 2). Pricing models with reservation or subscription were not considered here, since in this case the hiring of server capacities would be more favorable [e.g. 23].

\subsection{Results for ArcEGMO model runs}

Figure 2 shows the calculation times utilized by each provider in general and in comparison to the local computer. It is noteworthy that the virtual machines consume more time than the local device. These differences arise mainly during the simulation phase. This suggests that the pure computing power of technologically similar cloud instances is less than that of a corresponding local computer. Reading and writing processes on the hard disk during the model initialization are thus characterized by a very similar consumption of time.

However, if instances of the latest hardware generation can be applied, as provided by Amazon, there will be a significant performance improvement (Figure 3).

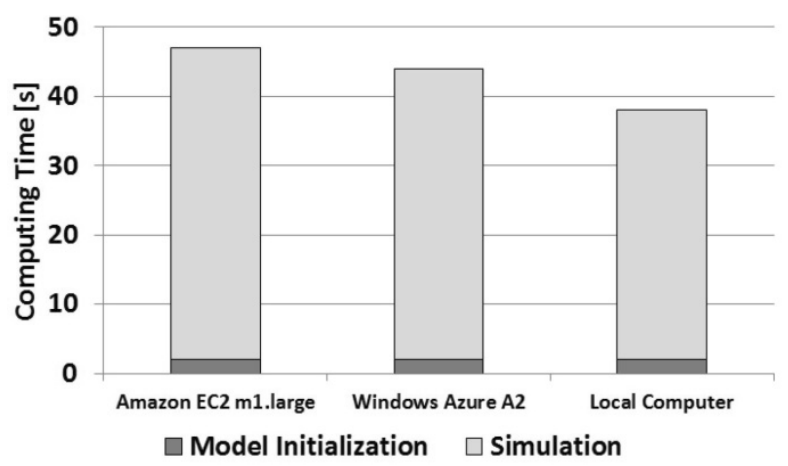

Figure 2. Computing time of ArCEGMO on different platforms.

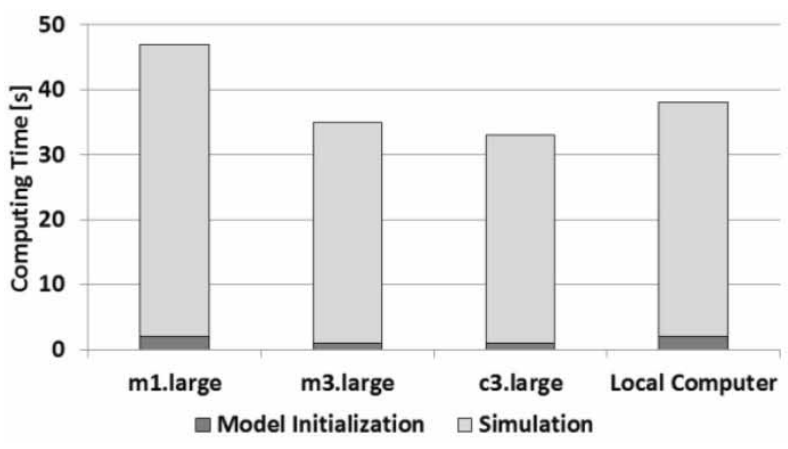

Figure 3. Differences between the Amazon EC2 instance types and the local machine in the required computing time.

If the number of cores per instance is increased, the speed of computing, as expected, cannot be increased, since these additional resources are not applicable due to the sequential programming of the model (results are not presented here). Surprisingly, in Amazon's pricing model, the acquisition of the latest processor hardware is cheaper than using older generations [16; Figure 4]. 


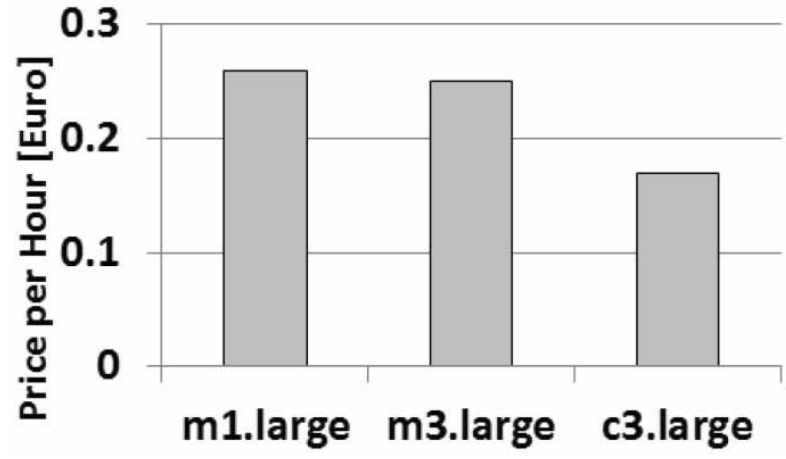

Figure 4. Price per hour for different Amazon EC2 instance types with no additional fees.

Considering Amazons 'on demand' prices without reservation on a monthly basis $(180 €$ and $125 €$ for $\mathrm{m} 3$.large and c3.large), long term use is still too expensive. For less complex areas where the overall computation time is small, the monetary costs without additional storage and communication services are below 20 cents per hour for an instance with the latest and most powerful computing hardware.

However, if large, highly spatially resolved models are calibrated so that the computing power and memory demands increase drastically for several days, costs are quickly increased. If, in that case Amazon instances equipped with the latest hardware generation are applied, one can still expect good operation performance of the model compared to a local device.

\section{Conclusion and Outlook}

Flood water simulations must be reliable in order to prevent losses and therefore need to be based on well calibrated rainfall runoff models of sufficient complexity. Using the hydrological modeling system ArcEGMO, it could be demonstrated that multiple model calculations, as necessary for model calibration, can also be implemented on virtual machines in IaaS clouds. However, the computing power is slightly below that of a comparable local computer with similar hardware. The outsourcing of model calculations is only reasonable if the latest computer hardware generation is offered by IaaS providers. The costs incurred thereby are low, especially for models with little complexity.
Due to the sequential structure of the modeling system ArcEGMO, additional processor cores cannot yet be exploited for a model run, which is why only a small increase in performance through improved processor technology in the cloud can be achieved. In this case, cloud computing is currently only an alternative in the particular circumstances of a shortterm, increased computational demand, which can not be fulfilled by local computing resources.

The trend of recent years towards technologies of software parallelization will have an influence on the development of novel hydrologic models. The conversion of the model system ArcEGMO towards the integration of multiple cores is currently tested by the authors. The advantage is that calculations can be distributed to multiple cores, which reduces the computing time per simulation depending on the proportion of parallelizable source code. Disadvantages are the major programming effort and the greater need for technical expertise. Existing code is not indefinitely parallelizable, so cloud computing will remain attractive in the long run, since parallelization is well supported due to the scaling of virtual hardware and since the pricing levels are expected to decrease.

Basically, there are further possibilities to decrease the amount of time required for model calibration. Optimization methods for automated model calibration, for example, can be instructed to distribute the individual model runs during calibration on multiple processor cores and to assume the management of the model runs, if supported. Correspondig studies by the authors, which also take cluster computers into account, are in preparation.

\section{Acknowledgements}

This study was created as part of the 'EEnVEB' project. The project is funded by the European Union (European Social Fund).

\section{References}

[1] Bates B, Kundzewicz ZW, Wu S, Palutikof J: Climate Change and Water. IPCC Technical Paper VI, Intergovernmental Panel on Climate Change (IPCC, Geneva). www.ipcc.ch, 2008.

[2] Gesamtverband der Deutschen Versiche rungswirtschaft: Erste Schadensbilanz: Hochwasser 2013 verursacht 180000 versicherte Schäden in Höhe von fast 2 Milliarden Euro. Pressemitteilung. Berlin, 2013. 
[3] Bornebusch M: Hydraulische und Hydrologische Modellsimulationen als Planungswerkzeug für HochwasserSchutz-Maßnahmen. Aachen, 2006.

[4] Maßmann S, Jakobs F, Sellerhoff F, Feldmann J, Sieker $\mathrm{H}$, Lange C, Om Y,Hinkelmann R: Hyd ${ }^{3}$ Flow - Integrierte hydrologische und hydro-numerische Mdellsysteme für eine verbesserte Hochwasservorhersage. Forum für Hydrologie und Wasserbewirtschaftung, "Tag der Hydrologie 2010", Braunschweig, 2010.

[5] Rouholahnejad E, Abbaspour KC, Vejdani M, Srinivasan R, Schullin R, Lehmann A: A parallelization framework for calibration of hydrological models. Environmental Modelling \& Software 31 (May), 2012.

[6] Moya Quiroga V, Popescu I, Solomatine DP, Bociort L: Cloud and cluster computing in uncertainty analysis of integrated flood models. Journal of Hydroinformatics 15(1), 2012.

[7] BITKOM: Cloud Computing - Evolution in der Technik, Revolution im Business. BIT-KOM-Leitfaden, Berlin, 2012.

[8] Pfützner B: Description of ArcEGMO; Official homepage of the modelling system ArcEGMO. www.arcegmo.de, ISBN 3-00- 022290-5, 2002.

[9] Pfützner B, Hesse P, Mey S,Klöcking B: N-AModellierung mit ArcEGMO. In: J. Diet-rich und M. Schöninger (Editors): Hydro-Skript. http://www.hydroskript.de, 2013.

[10] Pfützner B,Hesse P: Anwendung hydrologischer Modelle für die Hochwassserbemes-sung - Erfahrungen aus Sachsen-Anhalt. In: A. Schulte, C. Reinhardt, A. Dittrich, R. Jüpner und V. Lüderitz (Editors): Hochwasserdyna-mik und Risikomanagement - neue Ansätze für bekannte Probleme? Beiträge zum Gemeinsa-men Kolloquium am 24.11.2011 in Berlin. Be-richte aus der Geowissenschaft Freie Universi-tät Berlin, Aachen, 2011

[11] Büttner U, Thieming V, Lechtaler E,Pfützner B: Kap. 2 Untersuchungsgebiet. In: Schumann, A. (Editor): Entwicklungen integrativer Lösungen für das operationelle Hochwassermanagement am Beispiel der Mulde. Schriftenreihe Hydrologie / Wasserwirtschaft Ruhr-Universität Bochum, 2009.

[12] Tonninger W.: Die Cloud-Gretchen-Frage: IaaS oder PaaS? http://businessreadyblog.wordpress.com/2011/ 02/25/die-cloud-gretchen-frage-iaas-oder-paas/, 2011 (validated 11.3.2014).
[13] Walterbusch M, Teuteberg F: Datenverluste und Störfälle im Cloud Computing: Eine quantitative Analyse von Service Level Agreements, Störereignissen und Reaktionen der Nutzer. Proceedings: Multikonferenz Wirtschaftsinformatik, Paderborn, 2014.

[14] Deelman E, Singh G, Livny M, Berriman B, Good J: The Cost of Doing Science on the Cloud: The Montage Example. In: Proceedings of the 2008 ACM/IEEE conference on Supercomputing, article No. 50. IEEE Press, Piscataway, 2008.

[15] Berendes CI, Ertel M, Röder T, Sachs T, Süptitz T, Eymann T: Cloud Computing lohnt sich (noch) nicht. 11th International Conference on Wirtschaftsinformatik, Leipzig, 2013.

[16] Amazon Web Services: Amazon EC2 - Preise. aws.amazon.com/de/ec2/pricing, 11.3.2014.

[17] Amazon Web Services: Amazon EC2-Instances. aws.amazon.com/de/ec2/instance-types/, 11.3.2014.

[18] Microsoft: Windows Azure: Virtuelle Computer - Preisdetails. www.windowsazure.com/dede/pricing/details/virtual-machines/, 11.3.2014.

[19] Armbrust M, Fox A, Griffith R, Joseph AD, Katz R, Konwinski A, Lee G, Patterson D, Rabkin A, Stoica I, Zaharia M: A View of Cloud Computing. Communications of the ACM 53 (4), 2010.

[20] Matros R, Stute P, von Zuydtwyck NH,Eymann T: Make-or-Buy im Cloud-Computing - Ein entscheidungsorientiertes Modell für den Bezug von Amazon Web Services. Bayreuther Arbeitspapiere zur Wirtschaftsinformatik, Bayreuth, 2009.

[21] Hochstätter CH: Microsofts Sicht auf die Cloud: Windows Azure im Praxistest. www.zdnet.de/41554705/microsofts-sicht-auf-die-cloudwindows-azure-im-praxistest/, 2011 (validated 11.3.2014).

[22] Innova Handelshaus AG: www.innova24.biz/item/computer-und-navigation/pcsysteme/acer-desktop-veriton-s670g-intel-core2duoe7600-2gb-intel-g- 793994.htm, 2013 (validated 13.3.2014).

[23] Hetzner Online AG: www.hetzner.de/hosting/produktmatrix/rootserverproduktmatrix/, 2014 (validated 13.3.2014). 


\section{EUROSIM 2016 $9^{\text {th }}$ EUROSIM Congress on Modelling and Simulation}

City of Oulu, Finland, September 12 - 16, 2016
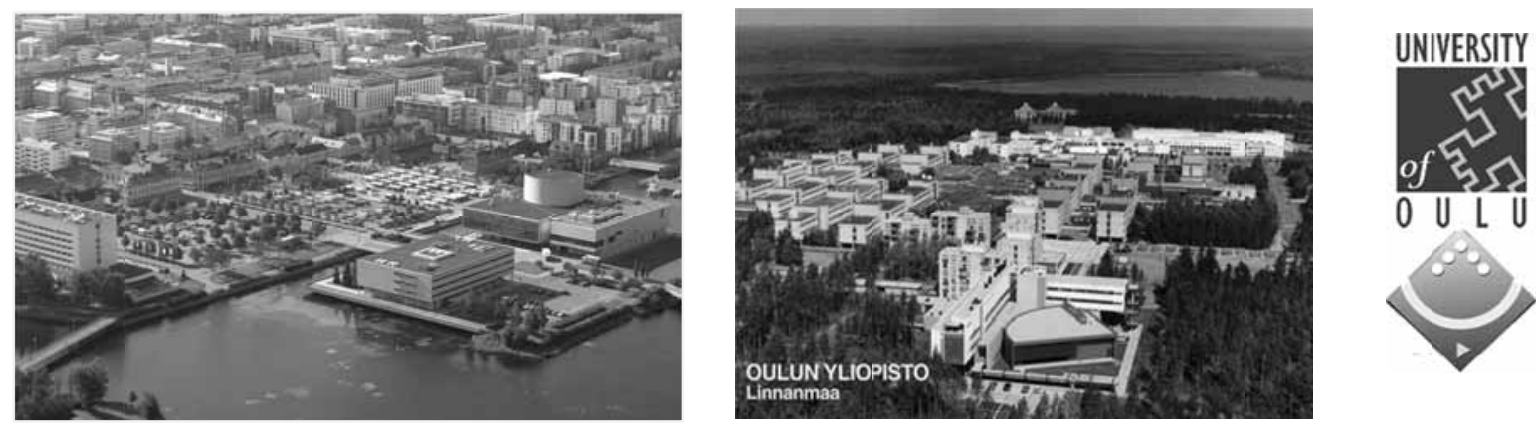

EUROSIM Congresses are the most important modelling and simulation events in Europe. For EUROSIM 2016, we are soliciting original submissions describing novel research and developments in the following (and related) areas of interest: Continuous, discrete (event) and hybrid modelling, simulation, identification and optimization approaches. Two basic contribution motivations are expected: M\&S Methods and Technologies and M\&S Applications. Contributions from both technical and non-technical areas are welcome.

Congress Topics The EUROSIM 2016 Congress will include invited talks, parallel, special and poster sessions, exhibition and versatile technical and social tours. The Congress topics of interest include, but are not limited to:

Intelligent Systems and Applications Hybrid and Soft Computing Data \& Semantic Mining Neural Networks, Fuzzy Systems \& Evolutionary Computation Image, Speech \& Signal Processing Systems Intelligence and Intelligence Systems Autonomous Systems Energy and Power Systems Mining and Metal Industry Forest Industry

Buildings and Construction Communication Systems

Circuits, Sensors and Devices

Security Modelling and Simulation
Bioinformatics, Medicine, Pharmacy and Bioengineering

Water and Wastewater Treatment, Sludge Management and Biogas Production

Condition monitoring, Mechatronics and maintenance

Automotive applications

e-Science and e-Systems

Industry, Business, Management, Human Factors and Social Issues

Virtual Reality, Visualization,

Computer Art and Games

Internet Modelling, Semantic Web and Ontologies

Computational Finance \& Economics
Simulation Methodologies and Tools Parallel and Distributed Architectures and Systems Operations Research Discrete Event Systems Manufacturing and Workflows Adaptive Dynamic Programming and Reinforcement Learning Mobile/Ad hoc wireless networks, mobicast, sensor placement, target tracking Control of Intelligent Systems Robotics, Cybernetics, Control Engineering, \& Manufacturing

Transport, Logistics, Harbour, Shipping and Marine Simulation

Congress Venue / Social Events The Congress will be held in the City of Oulu, Capital of Northern Scandinavia. The main venue and the exhibition site is the Oulu City Theatre in the city centre. Pre and Post Congress Tours include Arctic Circle, Santa Claus visits and hiking on the unique routes in Oulanka National Park.

Congress Team: The Congress is organised by SIMS - Scandinavian Simulation Society, FinSim - Finnish Simulation Forum, Finnish Society of Automation, and University of Oulu. Esko Juuso EUROSIM President, Erik Dahlquist SIMS President, Kauko Leiviskä EUROSIM 2016 Chair

Info: eurosim2016.automaatioseura.fi, office@automaatioseura.fi 


\title{
Decision Making in Ambient Assisted Living Environments based on Uncertain and Fuzzy Data
}

\author{
Dominik Brunmeir ${ }^{1 *}$, Johannes Kropf ${ }^{2}$ \\ ${ }^{1}$ Institute of Analysis and Scientific Computing, Vienna University of Technology, Wiedner Haupstraße 8-10, \\ 1040 Vienna, Austria * dominik.brunmeir@gmail.com \\ ${ }^{2}$ AlT Austrian Institute of Technology GmbH, Donau-City-Straße 1, 1220 Vienna, Austria
}

Simulation Notes Europe SNE 25(3-4), 2015, 179-184

DOI: $10.11128 /$ sne.25.tn. 10313

Received: September 16, 2015; Revised October 12, 2015;

Accepted: November 20, 2015

Abstract. A decision making engine for home automation in an assisted living context is presented. A Prototype was implemented, that will be integrated in HOMER, a middleware framework for home event recognition, as a reasoning component of the system. First tests show a reasonable error rate, which can be improved with careful selection of parameters.

\section{Introduction}

This article discusses a decision making engine in an Ambient Assisted Living (AAL) context. This decision making engine is not only able to learn and categorize human behavior by observing a person, but also replicate this person's actions. It recognizes altering behavioral patterns and adapts its actions to those new or changed situations. Its purpose is to automate certain activities to enhance the quality of life of elderly persons or persons with special needs.

Ambient Assisted Living is a growing field of research, as we are facing a severe demographic shift in Europe (see [1] and [2]). Better health care leads to higher life expectancy and therefor significantly more people in need of assistance in their later stages of life.

Provided there is a constant number of caregivers, this leads to either more work for them, or less support for those in need of care. To compensate for this, it is a necessity to increase the efficiency of the support or keeping the elderly independent of supporting measures.

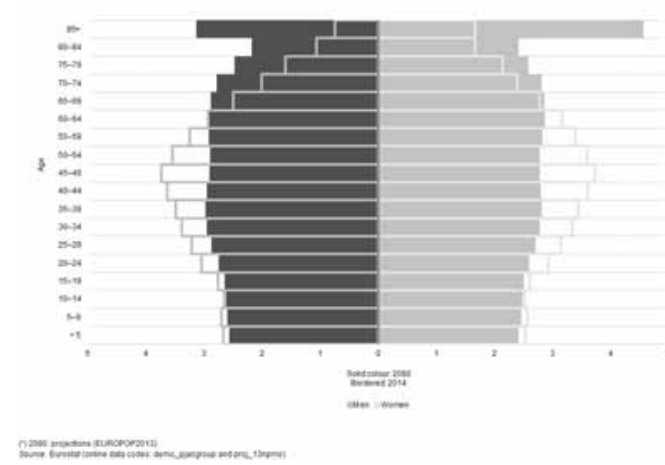

Figure 1: Change of population.

AAL tries to make progress on both issues (see [3]). AAL is a general term of different supportive techniques, that can be categorized in the following way [3]:

- Sensing

- Reasoning

- Acting

- Communication

- Interaction

Sensing includes every system that collects data. This includes on-body sensors or sensors in the environment of the person. Reasoning means processing the collected data, while acting is automatic control of actuators or other feedback mechanisms. This is in contrast to communication and interaction, where a reasoning component is included. The presented decision making engine is a reasoning component.

\section{Decision Making}

Decision making is the process of estimating the consequences of making a decision and picking the more desirable or better choice. 
There are two main issues with decision making: First we need to define and quantify the meaning of better, then we need set a framework for the decision making process itself. In our case, better means more similar to the desired behavior of an inhabitant, while the framework should imitate general human behavior.

Finite-state machines provide a framework to provide rule based automation, but as the number of sensors increases, they become more and more difficult to maintain in a consistent manner. It's also difficult to represent human behavior with such a strict (crisp) ruleset.

Another approach is the Adaptive Neuro-Fuzzy Modeling (ANFIS), which is a fuzzy inference system and better suited in learning and reproducing human behavior ([4]). It's aim is to generate an if-then rulebase with the help of artificial neural networks. Artificial neural networks were motivated by real neural networks and their ability to adapt to many problems. It consists of many connected simple units that work in parallel. The drawback of this approach is computational complexity and the difficulty of online adaptation of the rulebase, as the neural network would have to be rebuilt in case a new rule is discovered. While AOFIS generates easily interpretable membership functions with intuitive linguistic variables, ANFIS is not able to do so. For a performance comparison between AOFIS and ANFIS see [5], where it is shown, that AOFIS performs slightly better than ANFIS while also being able to adapting to user input online.

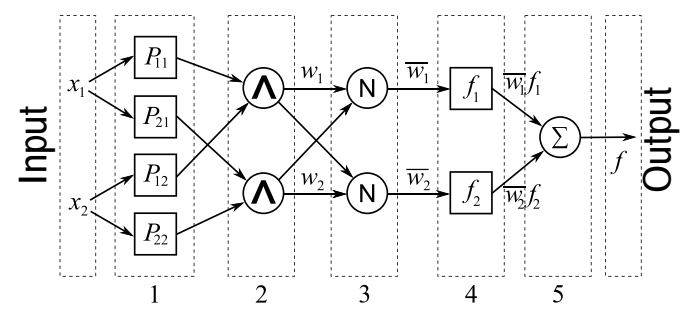

Figure 2: Schematics of ANFIS.

\section{AOFIS}

The Adaptive Online Fuzzy Inference System (AOFIS) was chosen because of its desirable properties. It shares certain similarities to fuzzy control systems, and thus is able to reproduce human behavior, which by itself is a strictly nonlinear problem (see [6], [7], [8] and [9]). AOFIS is also able to adapt during run time to changing behavior. It is based on a double-clustering tech- nique, which extracts interpretable fuzzy granules. As part of this work, it was implemented in Java for further integration in the HOMe Event Recognition System (HOMER).

AOFIS itself consists of five stages of operation:

- Collection of Data

- Extraction of Membership Functions

- Extraction of Fuzzy Rules

- Application of Fuzzy Rules

- Adaptation of Output

\subsection{Collection of data}

The data used in the experiments are taken from various sensors and actuates in an sample smart home. The input (sensor data) is stored in a line vector $X=$ $\left(x_{1}, x_{2}, \ldots\right) \in \mathbb{R}^{n}$, as is the output data (the actuators), $Y=\left(y_{1}, y_{2}, \ldots\right) \in \mathbb{R}^{m}$. Further we will consider the combined line vector $R=\left(x_{1}, \ldots, x_{n}, y_{1}, \ldots, y_{n}\right) \in \mathbb{R}^{n+m}$.

\subsection{Extraction of membership functions}

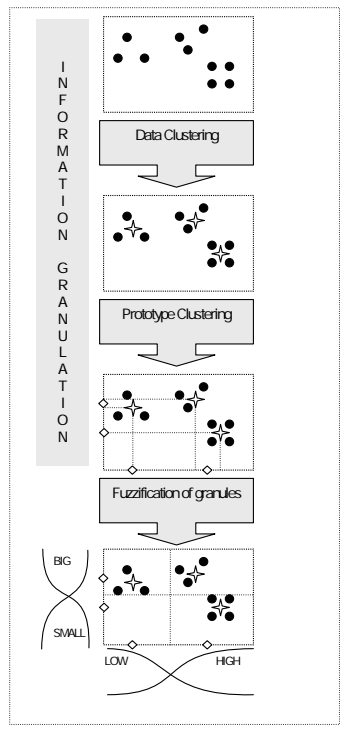

Figure 3: Extraction of Membership Functions schematics.

The Membership functions for AOFIS are generated by a double-clustering technique and extraction of so called fuzzy granules. The first clustering is performed in the $\mathbb{R}^{n}$ space, while the second one is along each dimension of the input space. 


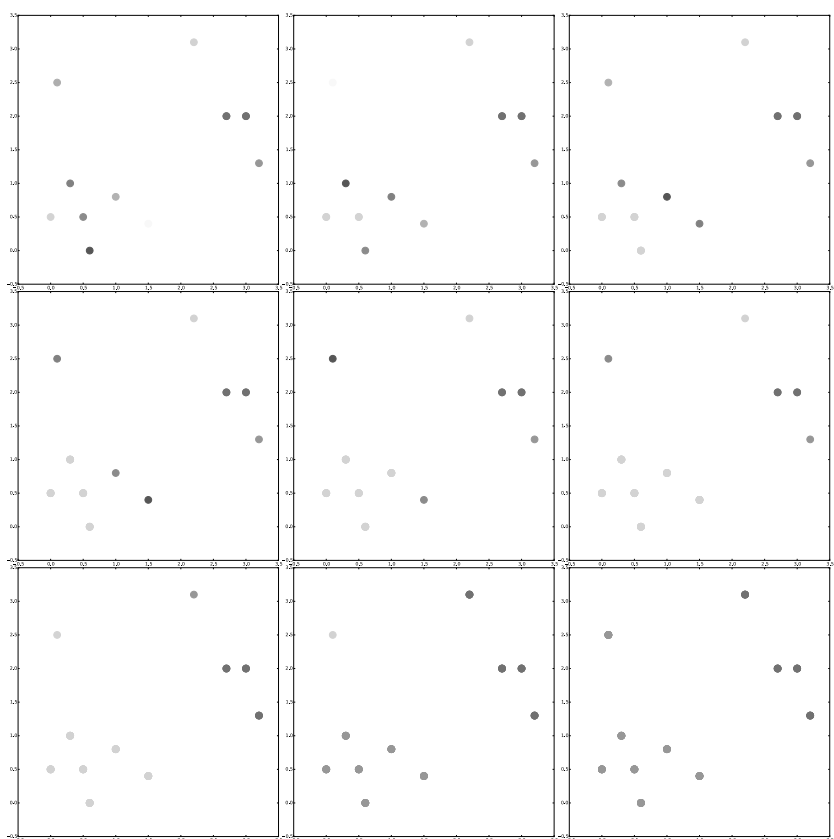

Figure 4: Agglomerative Hierarchical Clustering.

The first clustering algorithm is the Fuzzy c-Means Algorithm (FCM), which was discovered and described by Bezdek (see [10]) . FCM is a well known fuzzy clustering algorithm, where we minimize the following function to obtain cluster centers:

$$
J=\sum_{j=1}^{k} \sum_{x_{i} \in S_{j}}\left(u_{i j}\right)^{m} d\left(x_{i}, c_{j}\right)^{2}
$$

where $c_{j}$ is the $\mathrm{j}$-th cluster center, $x_{i}$ the $\mathrm{i}$-th data vector and $d$ a suitable (inner product-induced) norm. $u_{i j}$ is the membership matrix, where we have the membership of every point $x_{i}$ to cluster $c_{j}$.

The second clustering algorithm is an agglomerative hierarchical one. It starts with all points in a separate cluster, then takes the the two nearest clusters and merges them until the desired number of clusters is attained.

In case of our collected date, the input of the first algorithm is our input data in $\mathbb{R}^{n}$. The output are $j \mathrm{n}$ dimensional cluster centers. The input of the second algorithm are $j . n$ one dimensional points, built from coordinates of the cluster centers, with $k$ cluster-centers.

The next step is to fuzzify the information granules that we extracted with the double-clustering. First we partition our input space along the center of two neighboring cluster centers $c_{i}$ :

$$
t_{i}^{j}= \begin{cases}2 \cdot \min \left(c_{i}^{j} \mid c_{i}^{j} \in C_{j}^{l}\right)-t_{1}^{j} & i=0 \\ \frac{c_{i}+c_{i-1}}{2} & 0<i<l_{j} \\ 2 \cdot \max \left(c_{i}^{j} \mid c_{i}^{j} \in C_{j}^{l}\right)-t_{k-1}^{j} & i=l_{j}\end{cases}
$$

Now we can describe the input space with fuzzy sets. The chosen membership function is the gaussian function:

$$
g(x)=e^{\left(\frac{-(x-\mu)}{\sigma}\right)^{2}}
$$

where $\mu$ and $\sigma$ are calculated as follows:

$$
\begin{aligned}
& \mu_{i}^{j}=\frac{\left(t_{i-1}^{j}+t_{i}^{j}\right)}{2} \\
& \sigma_{i}^{j}=\frac{t_{i}^{j}-t_{i-1}^{j}}{2 \sqrt{-2 \ln \varepsilon}}
\end{aligned}
$$

Those sets are also easily interpretable with linguistic variables such as high, medium or low.

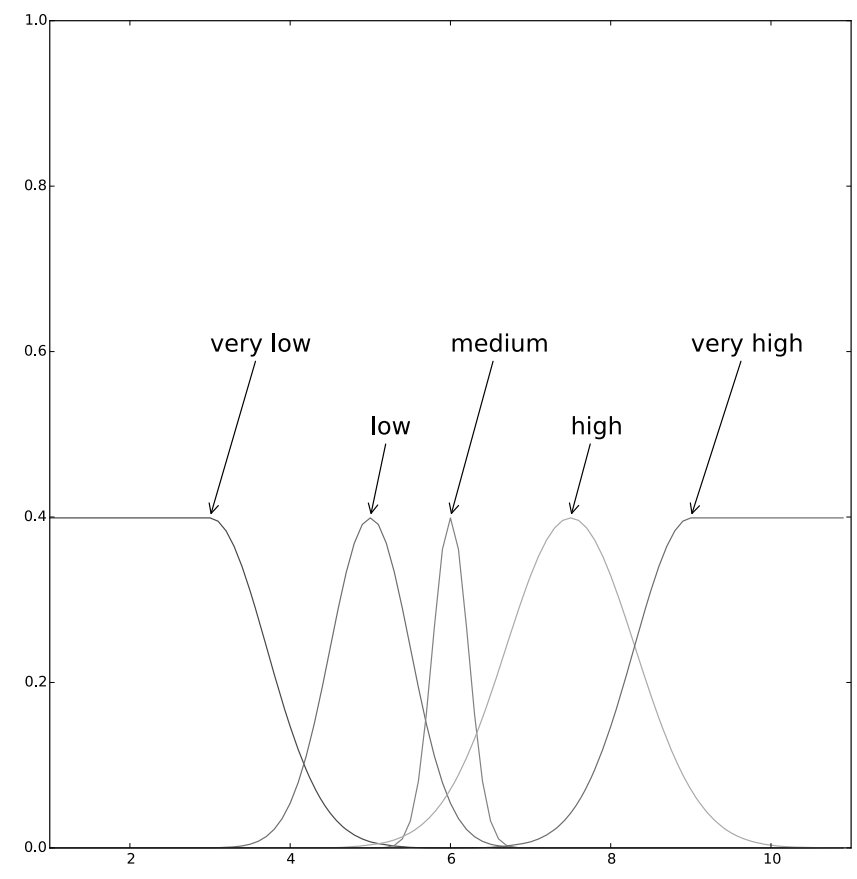

Figure 5: Linguistic Variables.

\subsection{Extraction of fuzzy rules}

A fuzzy or rule has the following general structure:

$$
\begin{aligned}
& R_{i}: \text { IF } x^{1} \text { is } A_{i}^{1} \text { AND } \ldots \text { AND } x^{n} \text { is } A_{i}^{n} \\
& \text { THEN } y^{1} \text { is } B_{i}^{1} \text { AND } \ldots \text { AND } y^{m} \text { is } B_{i}^{m}
\end{aligned}
$$


$x^{j}$ are the input variables and $y^{j}$ the output values, while $A_{i}^{j}$ and $B_{i}^{j}$ are the corresponding fuzzy sets. The set of all fuzzy rules is called a rule base. In our case the input variables is the measured sensor data, while the output values are the actuators. The fuzzy sets are the previously extracted membership functions

If we apply this scheme to the collected data, conflicting rules will be generated. To build a consistent rule base those conflicting rules have to be combined in some way. The method used is a weighted mean of all generated conflicting rules:

$$
\tilde{y}=\frac{\sum_{t_{k}=1}^{k} y_{t_{k}} w_{t_{k}}}{\sum_{t_{k}=1}^{k} y_{t_{k}}}
$$

$t_{k}$ denominates all conflicting rules with the same antecedent, and $w_{t_{k}}$ the weight of the $t_{k}$-th rule, with $w_{t_{k}}$ as the product of all memberships of $x_{t_{k}}^{j}$ in $A^{j}$

$$
w_{t_{k}}=\prod_{j=1}^{n} \mu_{A^{j}}\left(x_{t_{k}}^{j}\right)
$$

\subsection{Application of fuzzy rules}

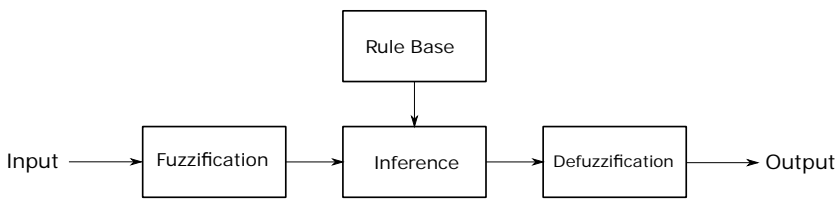

Figure 6: Procedure of the System.

With the help of the now extracted rules, the system may now control the habitat. To generate a crisp output from a crisp input, the following formula is applied:

$$
y=\frac{\sum_{l=1}^{M} \bar{y}^{l} \prod_{j=1}^{n} \mu\left(x_{j}\right)}{\sum_{l=1}^{M} \prod_{j=1}^{n} \mu\left(x_{j}\right)}
$$

\subsection{Adaptation of output}

This system is able to imitate a person's behavior in an habitat, but this behavior may be subject to changes as different needs arise. The person has to be able to override unwished control responses by manually controlling the system. AOFIS is able to register this and adapt its rules or add new ones. With the implementation of a delay, it is possible to filter out one-off actions.

If a manual control happens, the rule adaption routine compares each of the new input variables to those in the rule base. If a rule would have fired, the then part is adapted to the one, the user would have preferred, which is the one with the highest output membership value.

In case none of the existing rules would have fired, a new rule with the highest membership values for the input and the output is added.

\section{Implementation and Results}

\subsection{Implementation}

AOFIS was implemented in Java with the help of the Apache-commons-math library (see [11]) to ease integration into HOMER (see [12] for more information). The FCM and the agglomerative hierarchical clustering Algorithm had to be implemented, because at the time of writing, these algorithms were not available in the used library. The implementation was tested with various sample data sets [13] to ensure its functionality. One significant difference to the published AOFIS approach was the introduction of a threshold for replacing existing rules. We only replaced rules, that had more than 0.05 weight in the final output rule. This was done because with our sample data, AOFIS tended to also replace rules that didn't contribute much to the final output.

\subsection{Results}

The system is not yet implemented in HOMER, so only synthetic test could be performed. For the performance of using the system in a real smart home setting we have refer to [5] and [14].

For testing the system, we used real life data from 18 sensors in a smart home. This data set was labeled with different activities of daily life.

From this data set of 9896 input/output pairs, 3000 were taken randomly to train the system and build an initial rule base. The remaining pairs were fed to the system and whenever a mismatch between the output and the fired rule was registered, the system was adapted to simulate a manual override by the user. As parameters we were using 55 initial clusters and 9 double clusters.

As a result we were labeling almost $64 \%$ of the activities correctly. Most of the time the NULL label was incorrectly classified, followed by preparing food. Further analysis of the generated date showed, that the majority of these errors were caused by cases, which were 


\begin{tabular}{ll}
1 & NULL \\
\hline 2 & Comb Hair \\
\hline 3 & Wash Hands \\
\hline 4 & Wash Face \\
\hline 5 & Brush Teeth \\
\hline 6 & Make Bed \\
\hline 7 & Change Clothes \\
\hline 8 & Put Blinds up/down \\
\hline 9 & Prepare Food \\
\hline 10 & Eat \\
\hline 11 & Open/Close Window \\
\hline
\end{tabular}

Table 1: Activities of daily life.

\begin{tabular}{cccccc}
1 & 2 & 3 & 4 & 5 & 6 \\
\hline 638 & 87 & 74 & 73 & 233 & 297 \\
\hline \hline 7 & 8 & 9 & 10 & 11 & \\
\hline 90 & 233 & 589 & 139 & 38 & \\
\hline \hline
\end{tabular}

Table 2: Mismatched Labels using 9 double-clusters from 6896 inputs.

not yet covered by the initial rule base. These were classified as errors by the system, but showed, the system was still in the learning process to replicate the user's behavior. This is also the reason for the almost linear increase in accuracy, as those rules were added.

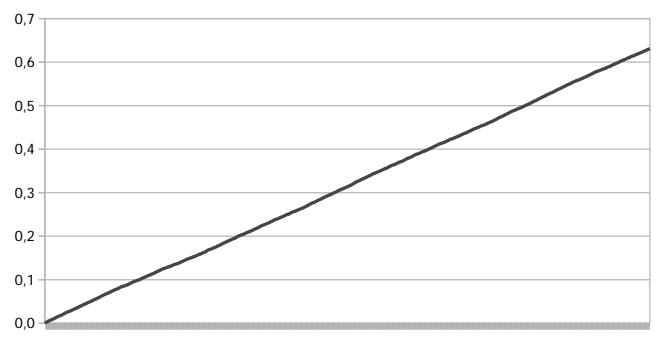

Figure 7: Ratio of mislabeled to correctly labeled data.

Our hypothesis was, that the rule base was too finegrained, but after testing with different sets of parameters (less clusters), the success rate became worse, with a higher ratio of overrides. This makes it clear, this system needs a relatively fine grained rule system but also a big enough training set to achieve high accuracy in its task. Given enough training data, at least a $90 \%$ rate of success is achievable (see [5]).

\subsection{Outlook}

The aim is to integrate this system into HOMER and test it with real-life data inside a smart home. It may also be interesting to examine the structure of the input data and apply certain techniques of data mining before feeding it to the system. As there are $18^{n}$ potential antecedents for the rule base ( $n$ is the number of double clusters), maybe some dimension reduction algorithms could be applied to preprocess the data (see [15]).

\section{Schematics of AOFIS Program}

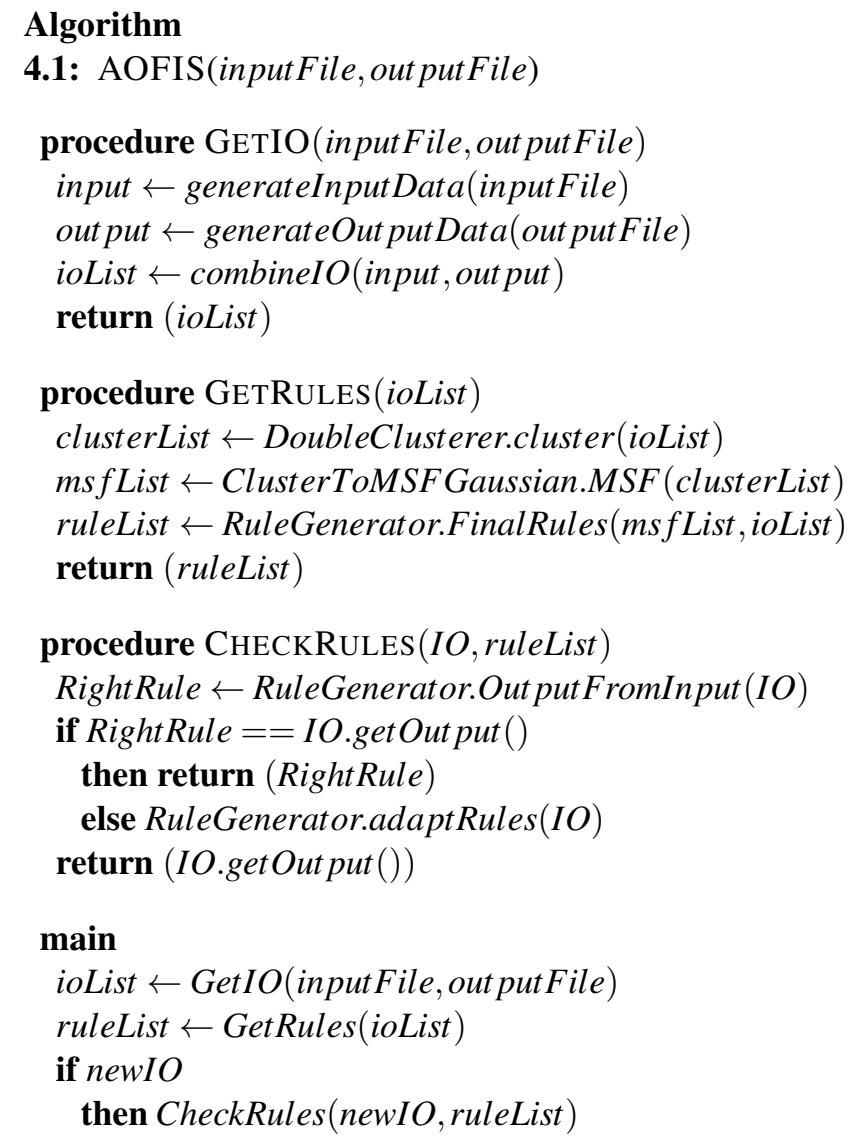

\section{References}

[1] Munstermann M. Technisch unterstützte Pflege von morgen. Springer. 2015.

[2] eurostat. Population and population change statistics. http://ec.europa.eu/eurostat/statisticsexplained/index.php/Population_and_population_ change_statistics. 
[3] Van Den Broek G, Cavallo F, Wehrmann C. AALIANCE Ambient Assisted Living Roadmap. Amsterdam, The Netherlands, The Netherlands: IOS Press. 2010.

[4] Jang J. ANFIS: Adaptive-Network-Based Fuzzy Inference System. IEEE Transactions on Systems Man and Cybernetics;.

[5] Doctor F, Hagras H, Callaghan V. An Intelligent Fuzzy Agent Approach for Realising Ambient Intelligence in Intelligent Inhabited Environments. IEEE Transactions on Systems, Man and Cybernetics, Part A: Systems and Humans. 2005;35:55 - 65.

[6] Zadeh L. Fuzzy Sets. Information and Control. 1965; 8:338-353.

[7] Zadeh L. Outline of a New Approach to the Analysis of Complex Systems and Decision Processes. IEEE Transactions on Systems, Man and Cybernetics. 1973; 3(1):28-45.

[8] Zadeh L. Towards a theory of fuzzy information granulation and its centrality in human reasoning and fuzzy logic. Fuzzy Sets and Systems. 1997;90:111-127.

[9] Cai K, Zhang L. Fuzzy Reasoning as a Control
Problem. IEEE Transactions on Fuzzy Systems. 2008; 16(3):600-614.

[10] Bezdek J. Pattern Recognition with Fuzzy Objective Function Algorithms. Springer. 1981.

[11] Apache. Commons Math. https://commons.apache.org/proper/commons-math/. 2015.

[12] AIT Austrian Institute of Technology GmbH Biomedical Systems. HOMER - Home Event Recognition System. http://homer.aaloa.org/. 2011.

[13] UCI. Machine Learning Repositorz. https://archive.ics.uci.edu/ml/datasets.html. 2015.

[14] Holmes A, Duman H, Pounds-Cornish A. The iDorm: Gateway to Heterogeneous Networking Environments. International ITEA Workshop on Virtual Home Environments. 2002;.

[15] Maimon O, Rokach L, eds. Data Mining and Knowledge Discovery Handbook. Springer. 2009. 


\title{
Expressing Requirements in Modelica
}

\author{
Lena Buffoni* , Peter Fritzson
}

Linköping University, SE-581 83 Linköping, Sweden; * lena.buffoni@liu.se

Simulation Notes Europe SNE 25(3-4), 185 - 189

DOI: $10.11128 /$ sne.25.tn. 10314

Received: Sept.10, 2015 (Selected SIMS 2014 Postconf. Publ.);

Accepted: October 20, 2015;

\begin{abstract}
As cyber-physical systems grow increasingly complex, the need for methodologies and tool support for an automated requirement verification process becomes evident. Expressing requirements in a computable form becomes a crucial step in defining such a process. The equation based declarative nature of the Modelica language makes it an ideal candidate for modeling a large subset of system requirements. Moreover, modeling both the requirements and the system itself in the same language presents numerous advantages. However, a certain semantic gap subsists between the notions used in requirement modeling and the concepts of cyber-physical modeling that Modelica relies on. To bridge this gap, in this paper, we illustrate through the use of dedicated types, pseudo function calls and function block libraries, how the Modelica language can be tailored to fit the needs of requirement modeling engineers
\end{abstract}

\section{Introduction}

Functional safety is a key concern in all industry sectors, be it nuclear plants, medical appliance manufactures or the automotive industry. The functional correctness of a component is the guarantee that the component behaves the way it should and fulfils all the functional requirements of the system. As the complexity of cyberphysical systems increases, maintaining coherent requirement specifications and using them to verify models of physical systems requires the formalisation of the requirements in a computable manner $[2,4]$. In this paper, we propose an approach to formalising the requirements in the same language as the model of the physical system.
For this purpose we choose Modelica, an objectoriented equation-based language for modeling multidomain physical systems [5, 1]. Expressing requirements in the same language as the physical model has numerous advantages. It improves the maintainability of the overall model, ensures that the requirements stay coherent as the model changes and simplifies the verification pro cess, as the requirements can be simulated together with the system model. However, engineers expressing requirements use domain specific terms and concepts [6]. Although requirement-specific notions can be expressed directly in Modelica, writing them from scrach every time manually can be complicated, and the resulting requirements can be harder to understand at first glance.

To bridge the gap between the requirement designer vision and the Modelica world, we define a set of types and pseudo functions, presented in the following section. A pseudo function is not a real function, since it allows side-effects and the use of time-dependent operators and equations in its body, which are disallowed in normal declarative Modelica functions. We extend Modelica with a mechanism for calling these pseudo functions, to simplify the readability of requirements. We illustrate these concepts on a simple example of a backup power system.

The paper is organized as follows. Section 1 introduces the notions used to map the requirements, Section 2 illustrates how the requirement verification is done, Section 3 discusses related works and finally Section 4 summarizes the article and discusses future works.

\section{Modelling Requirements}

In order to make the expression of requirements in Modelica as intuitive as possible in this section we introduce an approach of mapping concepts from the requirement modeling domain, such as those defined in [6] to the Modelica language. 


\subsection{Requirement type}

To treat requirements in a systematic manner, we need to define a dedicated requirement type. A requirement model should not influence the execution of the physical model, but only access the information from the physical model necessary for the requirement verification. Requirements are defined as special types of blocks: they have several inputs and a single output that represents the status of the requirement. A status can take the following values[11, 8]:

- violated when the conditions of the requirement are not fulfilled by the design model;

- not violated when the conditions of the requirement are fulfilled by the design model;

- undefined when the requirement does not apply, for instance a requirement that describes the behaviour of a power system when it is switched on, cannot be verified when the system is off.

If we take the example of a simple backup power system, which consists of several blocks connected in parallel and operates when the main power supply is lost, we can model a simple requirement 'When the power is on, the backup power-supply must not be activated', as follows in standard Modelica:

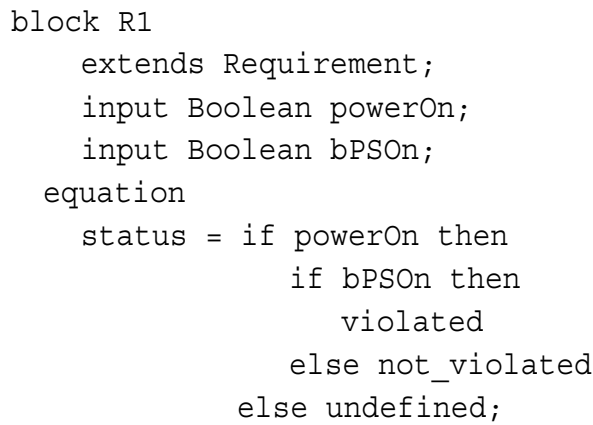

end R1;

In the case of such a simple requirement, no additional construct are necessary.

\section{2 'Pseudo function' library}

To bridge the semantic gap between the concepts used in requirement modeling and Modelica, we propose to define a set of Modelica function blocks to represent basic requirement modeling constructs. As mentioned, function blocks are a modified version of standard Modelica blocks, with a single output that can be called using a function syntax.
In particular, the time locator properties as defined in [6], such as after, WithinAfter, until, everyFor can be defined as Modelica function blocks. These constructs are used to which define a period in time when a requirement should be verified.

For instance everyFor (duration1, duration2), is a time locator that is used to define a requirement that must hold every duration1 seconds, for duration2 seconds.

Such constructs cannot be modeled as simple functions, as they are not context free and rely on time. Therefore to represent this everyFor, we can define the following Modelica function block:

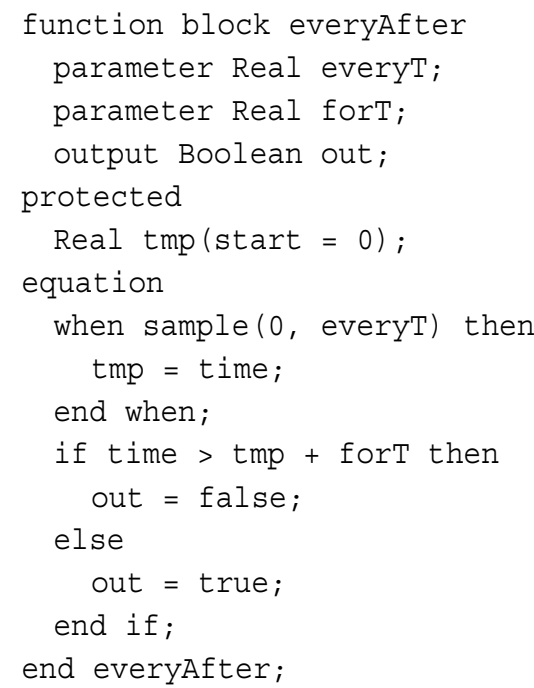

Requirements can then be expressed in terms of these basic building blocks in a more readable fashion. A set of predifined time locators based on the FORM-L specification is available, but the user can also define his own components.

\subsection{Anonymous function blocks through function calls}

If we take another simple requirement for a backup power unit, 'Within 40 seconds of the power being lost, at least two sets must be powered' and attempt to express it in Modelica, we will need to use the function block withinAfter, which is defined as follows: 


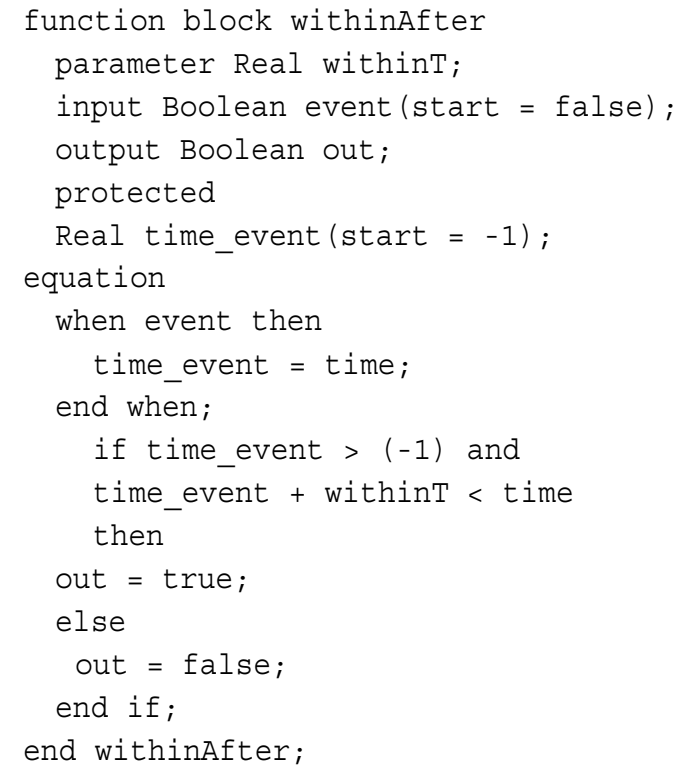

If we use standard Modelica blocks, then we need to explicitly create an instance of an withinAfter block and connect it to the corresponding inputs and outputs, which reduces the readability of the model. Therefore we propose to define a syntax for pseudo functions, where a function block can be called like a function by it's name and with parameters and input variables as arguments. We have implemented an extension in OpenModelica [7], that will automatically generate an instance of the required function block and the corresponding connection equations. With this syntax, we can define the above requirement in Modelica as follows:

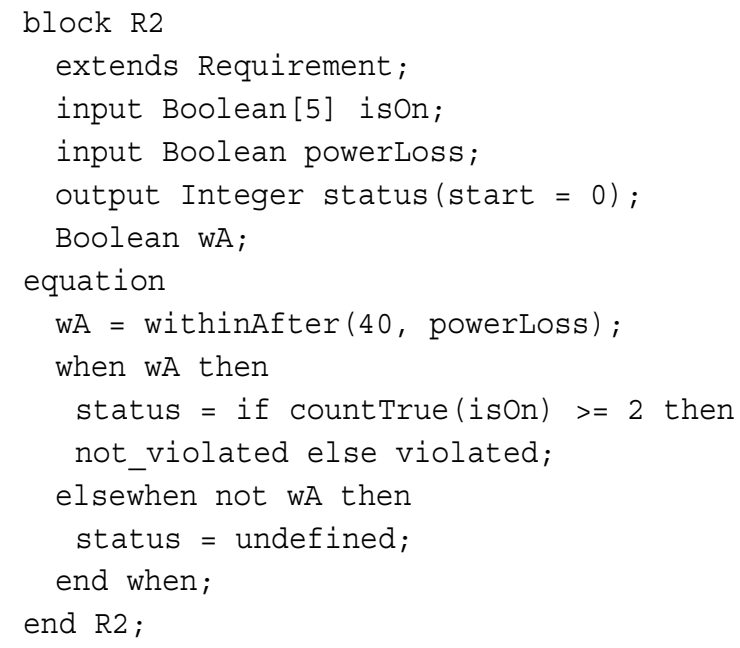

In this example, the function block withinAfter, is called as a function, and the arguments of the call represent the values that the function block should be instantiated with. The parameter withint should take the value 40 , and the signal powerLoss should be connected with the input event.

To generate this transformation we call the function rewriteFunctionBlockCalls (modelToRewrite, libraryPackage) in the OpenModelica API. This function will take two arguments, the model that needs to be rewritten and a package containing the function block definitions. It will then parse all the function calls, and replace all the calls to functions with the same names as the function blocks in the package passed in parameters with instantiations of the corresponding function blocks in the declaration section, and the result of pseudo function call will be the single output of the function block. The updated model is then reloaded into memory and can be simulated.

The argument passing works in the same way as for normal function calls, the positional instantiation will bind the values passed to the function call to the parameters and input variables of the function block in the order in which they are defined. Arguments can also be named explicitly, in which case the corresponding input value or parameter will be instantiated with the expression passed to the function. Saving a model after rewriteBlockCalls was called on it will generate standard Modelica code, for instance for the example above:

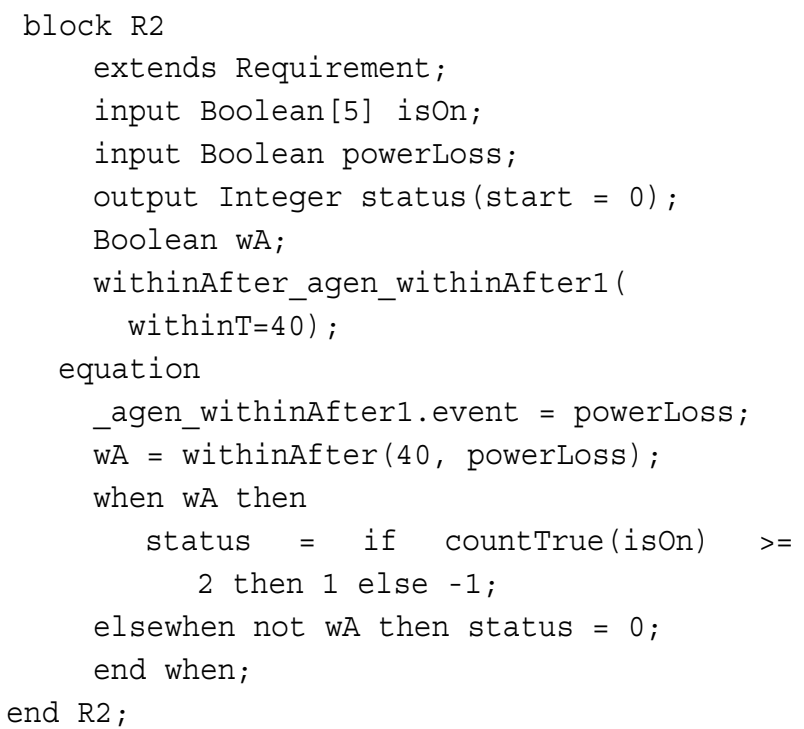


The extra step of generating standard Modelica code is important, as it allows to export the resulting models in standard Modelica, compatible with any Modelica tools, therefore function blocks are mapped to standard Modelica blocks.

It is improtant to destinguish between a requirement and a function block. The requirement maps to a system requirement, such as the one defined by R2 ('Within 40 seconds of the power being lost, at least two sets must be powered') and can contain one or more function blocks to represent time locators. As illustrated in the previous section, a requirement can also be independent of time and should then hold continuously.

\section{Requirement Verification}

Once the the requirement model and the system model are combined, they can be simulated together in order to verify the requirements. Each requirement has a status value which can subsequently be plotted to see at which times the requirement is violated.

The advantage of having the requirements in the same language as the system model is that no additional work is necessary to simulate the system. In the verification scenario in our example, the power is lost at time 20, and the back-up units 1 and 2 are turned on at time 40 (Figure 1). Therefore the requirement is not violated. The units 1 and 2 are turned off again at time 80, however since this behaviour does not affect the requirement, it remains not violated (Figure 2).

If we modify the verification scenario so that unit 2 is turned on at time 70 , the requirement will be violated as illustrated in Figure 3.

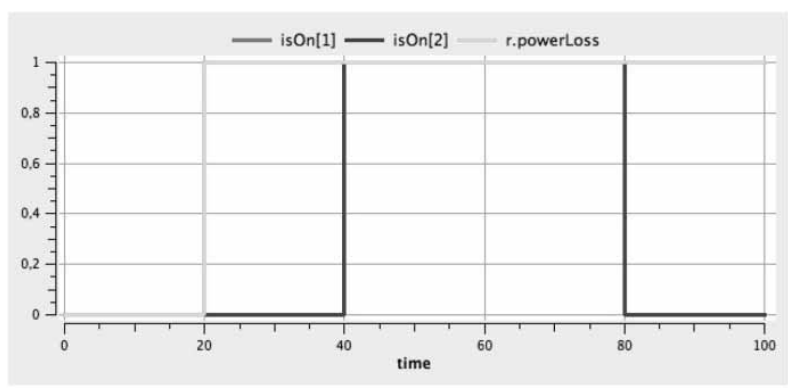

Figure 1. The power loss of the main power system and the switching on/off of backup units 1 and 2 .

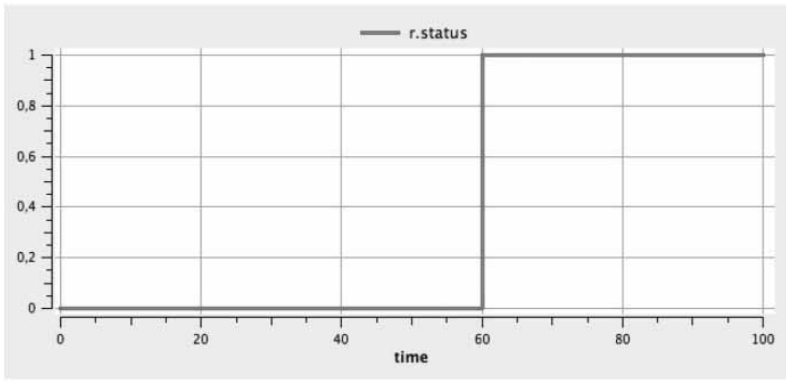

Figure 2. The requirement status, where 0 represents undefined, -1 violated and 1 not_violated.

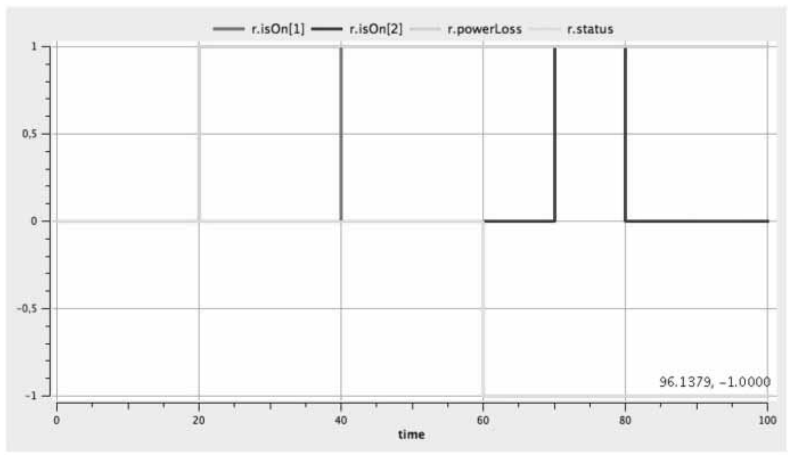

Figure 3. The requirement is violated because the power block 2 not turned on time.

\section{Related Work}

In this paper we have shown how textual require ments can be formalised in Modelica, however when dealing with large numbers of requirements and sim ulation scenarios, there is a need for an automated approach for composing the requirements with a given system design for the purpose of verification. In $[9,10]$ an approach for automating this process through the use of binding is proposed with an implementation in ModelicaML, a Modelica profile for UML. In [8] the requirement verification methodology is adapted to Modelica syntax. This work complements the work on formalising requirements in Modelica presented in this paper.

FORM-L language (FOrmal Requirements Modelling Language) is a language specification developed by EDF dedicated to expressing requirements and properties in a clear and concise manner [6]. In the work presented in this paper, concepts from FORM-L were mapped to Modelica function blocks in order to use them when modeling requirements in Modelica. 


\section{Conclusion}

In this paper we have illustrated how through a minimal set of extensions, we can use Modelica to formalise requirements and then verify them with respect to a specific system design.

Expressing requirements in the same language as the physical model brings the advantages of a modular, object-oriented language for system design to the process of requirement formalisation, and allows for a runtime verification of requirements. This work is part of a larger ongoing research project aiming to develop tool and methods [3] for model-driven, integrated system verification and fault analysis. Moreover, expressing the requirements in Modelica allows to formalise them and remove the ambiguity present in a verbal description.

The next step in this work is the integration with the work in [8] for an automatic generation of verification scenarios as well as tool support for batch processing of requirements.

\section{Acknowledgement}

This work is partially supported by the ITEA 2 MODRIO project.

\section{References}

[1] Fritzson P. Principles of Object-Oriented Modeling and Simulation with Modelica 2.1. Wiley IEEE Press, 2004.

[2] Hull E, Jackson K, Dick J. Requirements Engineering. Springer, 2005.

[3] ITEA 2 Projects. MODRIO. http://www.itea2.org/.

[4] Leucker and M, Schallhart C. A brief account of runtime verification. The Journal of Logic and Algebraic Programming, 78(5):293 - 303, 2009.

[5] Modelica Association. Modelica 3.2 revision 2 specification, 2013. www.modelica.org.

[6] Nguyen T. FORM-L: A MODELICA Extension for Properties Modelling Illustrated on a Practical Example. In Proceedings of The 10th International Modelica Conference, 2014.

[7] Open Source Modelica Consortium. Openmodelica project, 2013. www.openmodelica.org.

[8] Schamai W. Model-Based Verification of Dynamic System Behavior against Requirements. PhD thesis, Method, Language, and Tool Linköping: Linköping University Electronic, PressDissertations, 1547, 2013.

[9] Schamai W, Buffoni L, Fritzson P. An Approach to Automated Model Composition Illustrated in the Context of De-sign Verification. Modeling, Identification and Control, 35(2):79-91, 2014.

[10] Schamai W, Fritzson P, Paredis CJ. Translation of UML State Machines to Modelica: Handling Semantic Issues. Simulation, 89(4):498-512, April 2013.

[11] Tundis A, Rogovchenko-Buffoni L, Fritzson P, et al. Requirement verification and dependency tracing during simulation in modelica. In Proceedings of EUROSIM Congress on Modelling and Simulation, September 2013. 


\section{EUROSIM 2016 $9^{\text {th }}$ EUROSIM Congress on Modelling and Simulation}

City of Oulu, Finland, September 12 - 16, 2016
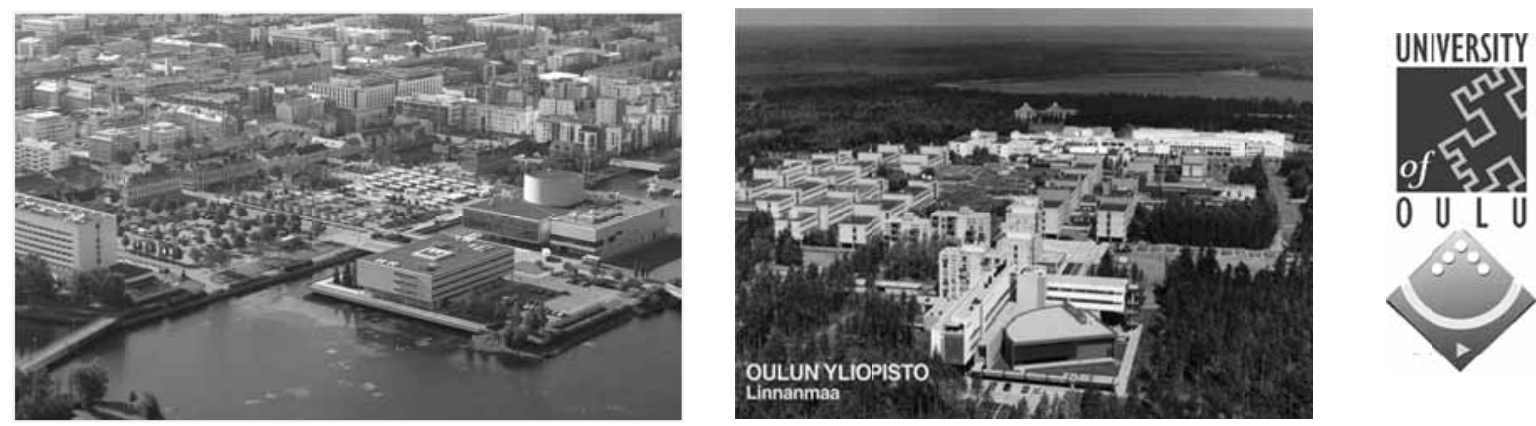

EUROSIM Congresses are the most important modelling and simulation events in Europe. For EUROSIM 2016, we are soliciting original submissions describing novel research and developments in the following (and related) areas of interest: Continuous, discrete (event) and hybrid modelling, simulation, identification and optimization approaches. Two basic contribution motivations are expected: M\&S Methods and Technologies and M\&S Applications. Contributions from both technical and non-technical areas are welcome.

Congress Topics The EUROSIM 2016 Congress will include invited talks, parallel, special and poster sessions, exhibition and versatile technical and social tours. The Congress topics of interest include, but are not limited to:

Intelligent Systems and Applications Hybrid and Soft Computing Data \& Semantic Mining Neural Networks, Fuzzy Systems \& Evolutionary Computation Image, Speech \& Signal Processing Systems Intelligence and Intelligence Systems Autonomous Systems Energy and Power Systems Mining and Metal Industry Forest Industry

Buildings and Construction Communication Systems

Circuits, Sensors and Devices

Security Modelling and Simulation
Bioinformatics, Medicine, Pharmacy and Bioengineering

Water and Wastewater Treatment, Sludge Management and Biogas Production

Condition monitoring, Mechatronics and maintenance

Automotive applications

e-Science and e-Systems

Industry, Business, Management, Human Factors and Social Issues

Virtual Reality, Visualization,

Computer Art and Games

Internet Modelling, Semantic Web and Ontologies

Computational Finance \& Economics
Simulation Methodologies and Tools Parallel and Distributed Architectures and Systems Operations Research Discrete Event Systems Manufacturing and Workflows Adaptive Dynamic Programming and Reinforcement Learning Mobile/Ad hoc wireless networks, mobicast, sensor placement, target tracking Control of Intelligent Systems Robotics, Cybernetics, Control Engineering, \& Manufacturing

Transport, Logistics, Harbour, Shipping and Marine Simulation

Congress Venue / Social Events The Congress will be held in the City of Oulu, Capital of Northern Scandinavia. The main venue and the exhibition site is the Oulu City Theatre in the city centre. Pre and Post Congress Tours include Arctic Circle, Santa Claus visits and hiking on the unique routes in Oulanka National Park.

Congress Team: The Congress is organised by SIMS - Scandinavian Simulation Society, FinSim - Finnish Simulation Forum, Finnish Society of Automation, and University of Oulu. Esko Juuso EUROSIM President, Erik Dahlquist SIMS President, Kauko Leiviskä EUROSIM 2016 Chair

Info: eurosim2016.automaatioseura.fi, office@automaatioseura.fi 


\title{
PDE Modeling with Modelica via FMI Import of HiFlow3 C++Components with Parallel Multi-core Simulations
}

\author{
Kristian Stavåker $^{1 *}$, Peter Fritzson ${ }^{1}$, Chen Song ${ }^{2}$, Martin Wlotzka ${ }^{2}$, Vincent Heuveline ${ }^{2}$ \\ ${ }^{1}$ Programming Environments Laboratory, Department of Computer and Information Science, Linköping University \\ Sweden; *Kristian.Stavaker@liu.se \\ ${ }^{2}$ Engineering Mathematics and Computing Laboratory, Interdisciplinary Center for Scientific Computing, \\ University of Heidelberg,Germany
}

Simulation Notes Europe SNE 25(3-4), 191 - 197

DOI: $10.11128 /$ sne. 25 .tn. 10316

Received: Sept.10, 2015 (Selected SIMS 2014 Postconf. Publ.);

Accepted: October 20, 2015;

Abstract. The Modelica modeling and simulation language is widely used in academia and industry to model complex, coupled dynamic systems which can be described by systems of ordinary differential equations (ODE) or differential algebraic equations (DAE). Recent work by the authors showed a way to enable partial differential equation (PDE) modeling with Modelica via functional mock-up interface (FMI) import of $\mathrm{C}+\mathrm{Com}$ ponents based on the multi-purpose finite element library HiFlow3. The finite element method (FEM) is largely used in both research and industry as a reliable technique for solving PDE problems.

In contrast to methods based on language extensions or automatic semidiscretizations in space, the approach with FMI import of HiFlow3 components into Modelica requires no change to the Modelica language, enables the use of specialized PDE solvers, and it allows for full flexibility in the choice of geometry, model parameters, and space discretization between simulation runs without recompilation. However, the computationally intensive PDE solving part in this approach can form a bottleneck in the simulations. In this work, we enhance the PDE solver by using a distributed memory parallelization based on a domain decomposition. As an example application, we consider a mechanical linear elasticity problem consisting of physical forces applied on a beam. Beams, plates and shells are common elements of solid structures with a sizable quantity of application in engineering design, appearing in fuselage, ship hulls, con- crete roof structure, etc. The derivation of elastic stress strain relations is a crucial point for mechanical analysis and validation, as the bending properties of the structure effects greatly the stability properties. In this work the actual beam is modeled and solved in parallel using a $\mathrm{C}++$ HiFlow3 component whereas the physical force acting on the beam is modeled using Modelica. We use the OpenModelica development environment but the same approach can be adapted to other Modelica environments.

\section{Introduction}

This paper is a continuation of the work in [20]. We discuss numerical simulation of models that couple partial differential equations (PDEs) and differentialalgebraic equations (DAEs) in the context of the Modelica modeling and simulation language $[14,5,6]$. Modelica originated around the idea of solving complex coupled dynamic systems, which can be described by systems of ordinary differential equations (ODE) or DAE. Up to now, there is only limited support for working with PDEs, despite the fact that the number of Modelica users in academia and in industry has lately grown significantly.

This paper is a continuation of the work in [20]. We discuss numerical simulation of models that couple partial differential equations (PDEs) and differentialalgebraic equations (DAEs) in the context of the Modelica modeling and simulation language $[14,5,6]$. Modelica originated around the idea of solving complex coupled dynamic systems, which can be described by systems of ordinary differential equations (ODE) or DAE. 
Up to now, there is only limited support for working with PDEs, despite the fact that the number of Modelica users in academia and in industry has lately grown significantly.

In this work, we propose a way to allow for PDE modeling with Modelica by importing $\mathrm{C}++$ components, written with the HiFlow3 multi-purpose finite element software [19], into Modelica using the Functional Mock-Up Interface (FMI) [15] import. FMI is a standard for model exchange and co-simulation between different tools. FMI supports only $\mathrm{C}$ but with correct linking it is possible to execute with $\mathrm{C}++$ code. We use the OpenModelica [3] development environment but the same approach can be adapted to other Modelica environments. As noted earlier, this paper is a continuation of the work in [20]. In that paper the heat distribution was computed for a copper beam. The heat source was controlled by a PID-controller. In this paper we continue to explore the Modelica-HiFlow3 coupling with parallel computations on multi-core architectures. We use a model of a steal beam with a force acting on it to demonstrate our implementation. We measure the elasticity deformation of the beam. In this work the actual membrane plate is modelled and solved in parallel using a $\mathrm{C}++$ HiFlow3 $[13,16]$ component whereas the physical force acting on the beam is modeled using Modelica.

The method described in this paper and in [20] has several advantages:

- HiFlow3 is well maintained and has strong support and capabilities for PDE modeling and solving;

- HiFlow3 and OpenModelica are free to download and use;

- The PDE structure is not lost but is maintained throughout the actual run-time simulation process. This allows for mesh refinement, solver run-time adjustments, etc.;

- It is possible to mix PDE and DAE systems in the same system setting. This is also possible in [10].

\section{Simulation Scenario}

We consider the deformation of a rectangular building element under a load. Figure 1 shows the setup of the configuration. The element is fixed at both ends, and the load is modeled by an external force acting on a part of the upper boundary.

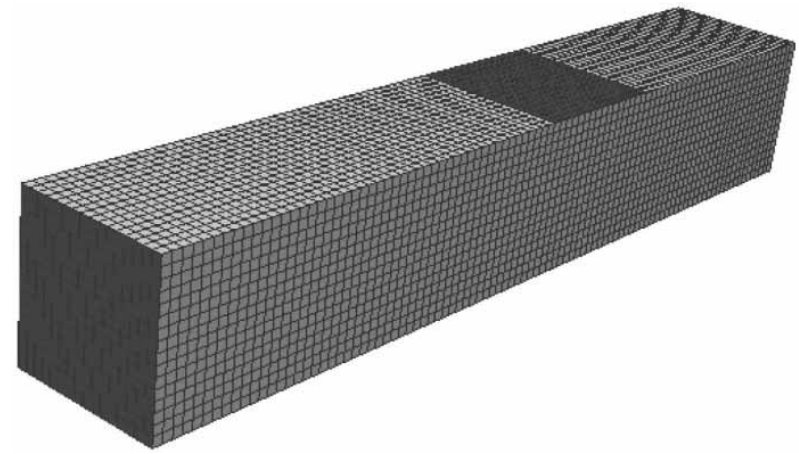

Figure 1. Geometry and computational mesh for the concrete element. The fixed front end is colored in blue, a force is acting on the red part.

\subsection{Linear elasticity model}

The element occupies a domain $\Omega \subset \mathbb{R}^{3}$ with boundary $\Gamma:=\partial \Omega$. The behaviour of the object subject to a force is described by means of the displacement $\boldsymbol{u}$ and the stress tensor $\sigma$. Conservation of momentum leads to the equilibrium equation

$$
-\nabla \cdot([I+\nabla u] \sigma)=\rho \boldsymbol{f} \text { in } \Omega
$$

where $\rho$ is the density of the material, $\boldsymbol{f}$ is a volumetric force, and $I$ denotes th identity matrix. In our scenario, gravity is the only volumetric force, therefore $\boldsymbol{f}=-g \boldsymbol{e}_{z}$.

According to Hooke's law for isotropic materials the stress tensor is related to the deformation tensor $\epsilon$ as

$$
\sigma=2 \mu \epsilon+\lambda \operatorname{tr}(\epsilon) I
$$

with material parameters $\mu$ and $\lambda$, known as the Lamé elasticity constants. Assuming small deformations, we neglect nonlinear terms in the deformation tensor resulting in the linearised form [2]

$$
\epsilon \approx \frac{1}{2}\left(\nabla u+\nabla u^{\top}\right)
$$

And also the deformation gradient is simplified as

$$
I+\nabla u \approx I .
$$

This leads to the following problem formulation: Find a function $u: \Omega \rightarrow \mathbb{R}^{3}$ as the solution of

$$
\begin{gathered}
-\mu \nabla \cdot\left(\nabla u+\nabla u^{\top}\right)-\lambda \nabla(\nabla \cdot u)=\rho f \text { in } \Omega, \\
u=0 \text { on } \Gamma_{0}, \\
{\left[\mu\left(\nabla u+\nabla u^{\top}\right)+\lambda \nabla(\nabla \cdot u) I\right] \cdot n=p \text { on } \Gamma_{1},} \\
{\left[\mu\left(\nabla u+\nabla u^{\top}\right)+\lambda \nabla(\nabla \cdot u) I\right] \cdot n=0 \text { on } \Gamma_{f} .}
\end{gathered}
$$


The homogeneous Dirichlet boundary condition (2b) fixes the beam at its ends. The load on the beam acts as a pressure $p$ through the Neumann boundary condition (2c), and the homogeneous Neumann condition (2d) is imposed on the free part of the boundary.

\subsection{Variational formulation}

A well-established method for numerically solving PDEs is the finite element method, which is based on a variational formulation of the system (2).

Assuming that there is a classical solution $u \in$ $C^{2}\left(\Omega, \mathbb{R}^{3}\right)$ of problem (2), equation (2a) is multiplied by a test function $v \in C_{0}^{\infty}(\Omega)$ and integrated over $\Omega$ :

$$
\begin{gathered}
-\mu \int_{\Omega}\left[\nabla \cdot\left(\nabla u+\nabla u^{\top}\right)\right] \cdot v d x-\lambda \int_{\Omega}\left[\nabla \cdot\left(\nabla u+\nabla u^{\top}\right)\right] \\
-\mu \int_{\Omega}\left[\nabla \cdot\left(\nabla u+\nabla u^{\top}\right) \cdot v d x\right] \\
-\lambda \int_{\Omega}[\nabla(\nabla \cdot u)] \cdot v d x=\int_{\Omega} \rho f \cdot v d x
\end{gathered}
$$

The divergence theorem yields (note that $\nabla v=\nabla v^{\top}$ )

$$
-\mu \int_{\Omega}\left[\nabla \cdot\left(\nabla u+\nabla u^{\top}\right)\right] \cdot c d x=\frac{\mu}{2} \int_{\Omega} \frac{\left(\nabla u+\nabla u^{\top}\right)}{\nabla v+\nabla v^{\top}} d x
$$

and

$$
-\lambda \int_{\Omega}[\nabla(\nabla \cdot u)] \cdot v d x=\lambda \int_{\Omega}(\nabla \cdot u)(\nabla \cdot v) d x,
$$

Where the boundary integrals are omitted since they vanish as $v=0$ on $\partial \Omega$. This leads to

$$
\begin{gathered}
\int_{\Omega} \frac{\mu}{2}\left(\nabla u+\nabla u^{\top}\right):\left(\nabla v+\nabla v^{\top}\right) \\
+\lambda(\nabla \cdot u)(\nabla \cdot v) d x=\int_{\Omega} \rho f \cdot v d x .
\end{gathered}
$$

For equation (3) to be well-defined, weaker regularity properties of $u$ and $v$ than in the classical context are sufficient. The problem can be formulated in terms of the Lebesgue space $\left[L^{2}(\Omega)\right]^{3}$ of square-integrable functions defined on $\Omega$ and with image in $\mathbb{R}^{3}$, and the Sobolev space $\left[H^{1}(\Omega)\right]^{3}$ of functions in $\left[L^{2}(\Omega)\right]^{3}$ with square-integrable weak derivatives.

We define the weak solution space

$$
V:=\left\{u \in\left[H^{1}(\Omega)\right]^{3}: u=0 \text { on } \Gamma_{0}\right\},
$$

the bilinear form

$$
\begin{gathered}
a:\left[H^{1}(\Omega)\right]^{3} \times\left[H^{1}(\Omega)\right]^{3} \rightarrow \mathbb{R} \\
a(u, v):=\int_{\Omega} \frac{\mu}{2}\left(\nabla u+\nabla u^{\top}\right):\left(\nabla v+\nabla v^{\top}\right) \\
+\lambda(\nabla \cdot u)(\nabla \cdot v) d x
\end{gathered}
$$

and the linear form

$$
\begin{aligned}
& l:\left[H^{1}(\Omega)\right]^{3} \rightarrow \mathbb{R}, \\
& l(v):=\int_{\Omega} \rho f \cdot v d x+\int_{\Gamma_{0}} p \cdot v d s .
\end{aligned}
$$

Note that the bilinear form a is symmetric, continuous and $V$-elliptic. Now we can state the variational formulation of problem (2):

Find $u \in V$ as the solution of

$$
a(u, v)=l(v) \quad \forall v \in V .
$$

This variational formulation admits a unique solution, which is called the weak solution of the elasticity problem.

\subsection{Finite element discretization}

Let $T_{h}:=\left\{K_{1}, \ldots, K_{N}\right\}$ be a triangulation of $\Omega$ with $N$ tetrahedron cells $K_{i}(i=1, \ldots, N)$. We define the finite element space of piecewise linear functions

$$
V_{h}:=\left\{v \in V:\left.v\right|_{K} \text { is linear }\left(K \in T_{h}\right)\right\} .
$$

$V_{h}$ has the finite dimension $n:=\operatorname{dim}\left(V_{h}\right)$. We give the problem formulation for a conforming finite element approximation of (4):

Find $u_{h} \in V_{h}$ as the solution of

$$
a\left(u_{h}, v_{h}\right)=l\left(v_{h}\right) \quad \forall v_{h} \in V_{h} .
$$

Let $\left\{\varphi_{1}, \ldots, \varphi_{h}\right\}$ be a basis of $V_{h}$. We define the ansatz function as:

$$
u_{h}(x):=\sum_{i=1}^{n} x_{i} \varphi_{i}(x)
$$

with coefficients $x_{i} \in \mathbb{R}$ and insert it into (5), yielding

$$
\sum_{i=1}^{n} x_{i} a\left(\varphi_{i}, \varphi_{j}\right)=b\left(\varphi_{j}\right) \quad(j=1, \ldots, n) .
$$

This can be written as the linear system

$$
A x=b,
$$

where

Is the stiffness matrix and

$$
A:=\left(a\left(\varphi_{j}, \varphi_{i}\right)\right)_{i, j=1, \ldots, n} \in \mathbb{R}^{n \times n}
$$

$$
b:=\left(l\left(\varphi_{i}\right)\right)_{i=1, \ldots, n} \in \mathbb{R}^{n}
$$

is the load vector. As the stiffness matrix is symmetric and positive definite [2], we employ the Conjugate Gradient (CG) method [7] for solving (6).

\section{Coupled Implementation}

More details of the coupled simulation setup can be found in [20]. 


\subsection{The Modelica modeling and simulation language}

Modelica is a language for equationbased objectoriented mathematical modeling which is being developed and standardized through an international effort in the Modelica Association [14]. The equation parts of Modelica requires a lot of the compiler developer: knowledge in compiler construction, symbolic manipulation of equations and associated mathematics, as well as knowledge of numerical mathematics. The simulation runtime system is also an important part and can be complex; various solver techniques for solving the differential equations can be applied. Modelica allows high-level concepts such as objectoriented modeling and component composition. Multidomain modeling is also possible in Modelica with the possibility of combining model components from a variety of domains within the same application. There exist several mature and wellmaintained Modelica development environments, such as Dymola, OpenModelica, MapleSim, Wolfram SystemModeler, Simulation X, and JModelica.org. See [5] and [6] for more information on the Modelica language.

\subsection{The OpenModelica environment}

OpenModelica is a modeling and simulation environment, which is developed and supported by an international consortium, the Open Source Modelica Consortium (OSMC) [3]. This effort includes an open-source implementation of a Modelica compiler, a simulator and a development environment for research, education and industrial purposes.

\subsection{The HiFlow3 Finite Element Library}

HiFlow3 $[19,1]$ is a multi-purpose finite element software providing powerful tools for efficient and accurate solution of a wide range of problems modeled by partial differential equations (PDEs). Based on object-oriented concepts and the full capabilities of $\mathrm{C}++$ the HiFlow3 project follows a modular and generic approach for building efficient parallel numerical solvers. It provides highly capable modules dealing with mesh setup, finite element spaces, degrees of freedom, linear algebra routines, numerical solvers, and output data for visualization. Parallelism - as the basis for high performance simulations on modern computing systems - is introduced at two levels: coarse-grained parallelism by means of distributed grids and distributed data structures, and fine-grained parallelism by means of platform-optimized linear algebra backends.

\subsection{HiFlow3-based PDE component}

HiFlow3 is a multi-purpose finite element method orientated mathematical library, it is well designed for modular and generic approach. Considering our application, HiFlow3's modules provide us an efficient computing platform [1] for things such as mesh setting, finite element function spaces, linear algebra structure, numerical solvers, etc. HiFlow3 also provides a welldefined parallel environment which allows to build up our elasticity solver as a robust component that we can execute on large scale machines with respect to high performance computing (HPC).

\subsection{Modelica model}

Our (relatively simple) Modelica model is shown in Listing 1. It contains the PDE component and the force variable. The variables $\mathrm{hf}$ Block. stateVar and $\mathrm{hf}$ Block. derstateVar do not do anything useful but are in the model because the OpenModelica runtime system needs at least one state variable to operate.

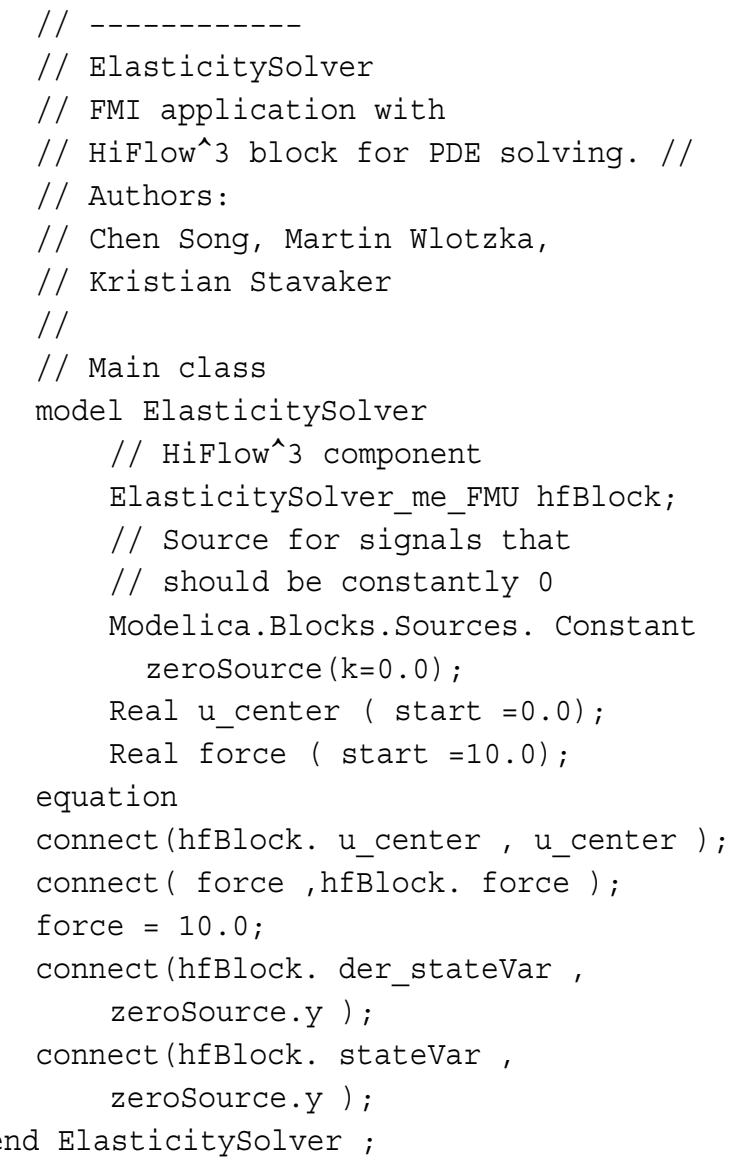

Listing 1. Modelica model. 


\section{Parallelization Concept}

The parallelization concept of HiFlow3 is based on a decomposition of the spatial domain into a number of subdomains. For distributed memory systems, the message passing interface (MPI) [4] is used for data transfer. Each MPI process is dedicated to the computation for one of the subdomains. Hereby, work is distributed among the processes. After creating the mesh, that our finite element discretization is based on, the METIS graph partitioner [9] is used to detemine a balanced partitioning of the mesh according to the number of MPI processes. Each process then only stores one part of the global mesh. Couplings between neighbouring parts are taken into account by means of a layer of ghost cells. Figure 2 shows an example of a domain decomposition into 8 parts. The matrix and vector data structures in HiFlow3 are distributed data structures which fit the partitioning imposed by the domain decomposition. Each process holds exactly those degrees of freedom of the finite element space which belong to its part of the domain. Couplings between different partitions are achieved by using ghost degrees of freedom. Only these have to be exchanged during parallel matrix-vectorproduct execution.

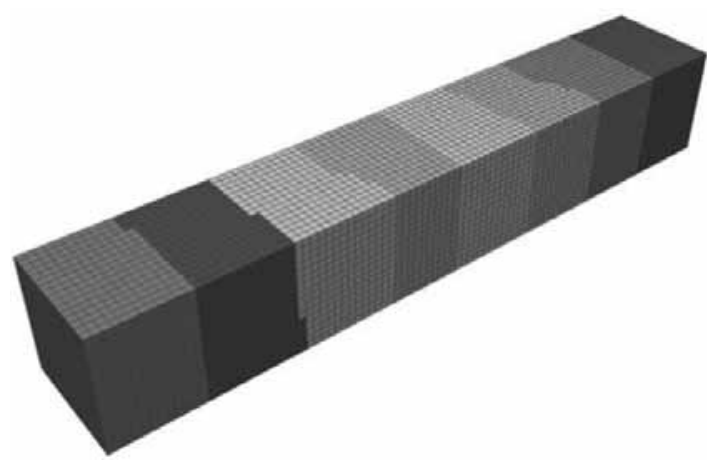

Figure 2. Patitioning of the mesh into 8 subdomains, indicated ba different color.

Assembly of the system matrix and right-hand-side vector, i.e. the computation of the entries, is done independently on each process for the corresponding subdomain. Hereby, the assembling process is designed in two levels: The global assembler iterates concurrently on each subdomain over the cells, while the local assembler computes the contributions for any single cell. Once the matrix and vector are assembled, the Conjugate Gradient linear solver takes advantage of the parallel implementation of the matrix-vector-operations when computing the solution.

\subsection{Parallel execution of the model}

The Modelica compiled model code is executed on a number of processes. The Modelica compiled model code is hereby replicated on each process. Whenever the HiFlow3 PDE component is called, it performs distributed parallel computations for solving the elasticity problem, hereby taking advantage of its parallelization concept based on the domain decomposition.

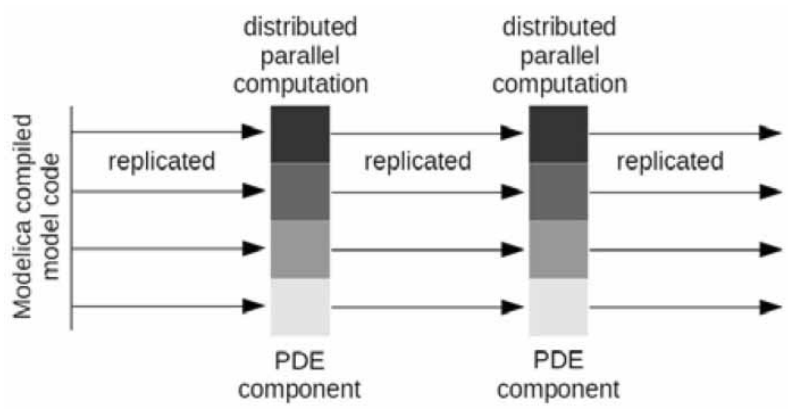

Figure 3. Replicated parallel execution of the Modelica compiled model code and distributed parallel computation in the HiFlow3 PDE component, sketched for 4 processes.

\section{Numerical Experiments}

We carried out a series of test runs with number of processes $n \in\{1,2,4,8,16\}$. We measured the runtime $T_{n}$ for the PDE component when running on $n$ processes.

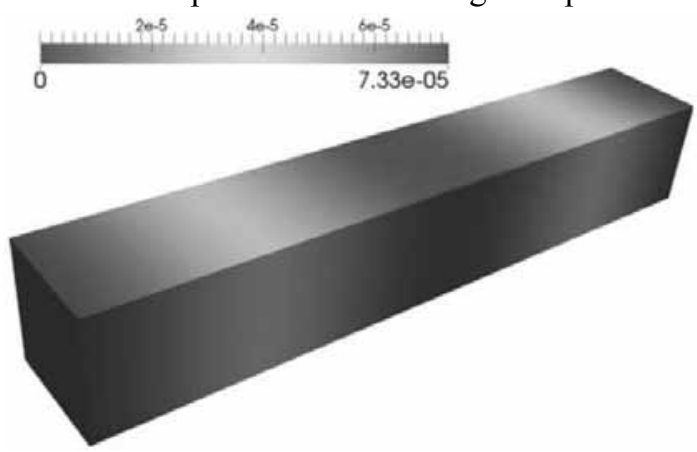

Figure 4. Visualization of the displacement in vertical direction.

To asses the parallel performance of the solver, we computed the speedup

and the efficiency

$$
S_{n}:=\frac{T_{n}}{T_{1}}
$$

$$
E_{n}:=\frac{S_{n}}{n}
$$

Where $T_{n}$ is the run-time of the solver when exevuted on $n$ MPI processes. Results are given in Table1. 


\begin{tabular}{llll}
\hline $\mathbf{n}$ & $\begin{array}{l}\text { run-time } T_{n} \\
{[\mathrm{sec}]}\end{array}$ & speedup $S_{n}$ & efficiency $E_{n}$ \\
\hline $\mathbf{1}$ & 8.830 & 1.0 & 1.0 \\
\hline $\mathbf{2}$ & 4.736 & 1.864 & 0.932 \\
\hline $\mathbf{4}$ & 2.948 & 2.995 & 0.749 \\
\hline $\mathbf{8}$ & 1.968 & 4.487 & 0.561 \\
\hline $\mathbf{1 6}$ & 1.741 & 5.072 & 0.317 \\
\hline
\end{tabular}

Table 1. Run-times for the PDE component with varying number of MPI processes.

Figure 5 shows a plot of the speedup and efficiency. The results show a parallel performance of the Hi-Flow3 PDE component within the Modelica context which is much poorer than the performance obtained for pure HiFlow3 applications. This is due to a technical reason: We needed to install a custom Open-MPI library, where we disabled the plugin architecture of the OpenMPI implementation on the machine while still using shared libraries. This was necessary for compiling the HiFlow3 PDE component into adynamic shared object which can be loaded by the Modelica compiled model code during runtime. The diminished parallel efficiency is clearly due to the use of such non-optimized MPI installation, since HiFlow3 shows good scalability on other machines with a high-performance MPI installation [8]. Nevertheless, we were able to leverage the parallel computing capabilities of HiFlow3 in the PDE component to introduce distributed memory parallelization for Modelica simulations. The performance tests show that even if parallel efficiency may not be optimal, our approach allows for solving large scale 3D PDE problems in high resolution on distributed memory machines. This is especially advantageous with respect to the amount of memory available, as the problem data can be split and distributed to several compute nodes, as opposed to a shared memory parallelization.

\section{Conclusions}

In this paper we have investigated a method of incorporating PDEs in the context of a Modelica model, by using FMI to import a PDE solver from the finite element library HiFlow3.
Numerical results obtained using a simple coupled model that invoke a force and measures the elasticity deformation of a beam demoefficient and with already validated software. This paper is a continuation of a paper from the SIMS 2013 workshop [20]. In that paper the distribution of heat was measured in a piece of copper. The heat source was controlled by a PID-controller. A novelty in this paper is that we make use of parallel computations. The main advantages of this type of coupling include its simplicity and the possibility to reuse existing solver technology on multi-core and distributed memory architectures. In the results section we provided some run-time measurements of these parallel computations and compared to single-core computations. Induced by the needs for compiling the PDE component into a dynamic shared object and loading it by the Modelica compiled model code, limitations in the MPI library influenced the parallel performance. However, the speedup obtained is considerable in simulation practice, and the use of distributed memory architectures is a clear advantage with respect to memory, especially for large scale problems. In combination with our previous work reported in [20], this opens opportunities to address even more compute- and memory-intensive applications like instationary fluid dynamics problems. The parallelization approach with replication might seem somewhat clumsy. A better method would perhaps be to let the OpenModelica run-time system drive the parallel distribution. This is future work and it should be noted that the FMI places limits on parallel communication.

\section{Acknowledgments}

Funded by the ITEA2 European Union MODRIO Project, by SSF in the EDOp project, and by the Swedish National Graduate School of Computer Science (CUGS).

\section{References}

[1] Anzt H, Augustin W, Baumann M, et al. Hiflow3 a multi-purpose and flexible parallel finite element package. pages 1-15, 2011.

[2] Dym CL, Shames IH. Solid Mechanics: A Variational Approach, Augmented Edition. Springer New York, 2013.

[3] The Open-Source OpenModelica Development Environment. http://www.openmodelica.org. 
[4] Message Passing Interface Forum. MPI: A MessagePassing Interface Standard, Version 3.0. HighPerformance Computing Center Stuttgart (HLRS), 2012. http://www.mpi-forum.org.

[5] Fritzson P. Principles of Object-Oriented Modeling and Simulation with Modelica 2.1. Wiley-IEEE Press, 2004.

[6] Fritzson P. Principles of Object-Oriented Modeling and Simulation with Modelica 3.3: A Cyber-Physical Approach. (to appear). Wiley-IEEE Press, 2014.

[7] Hestenes MR, Stiefel E. Methods of conjugate gradients for solving linear systems. Journal of research of the National Bureau of Standards, 49:409-436, 1952.

[8] Heuveline V, Ketelaer E, Ronnas S, et al. Scalability study of hiflow3 based on a fluid flow channel benchmark. 8th BFG/bw-GRID Workshop Proceedings, 2012.

[9] Karypis G, Kumar V. A fast and high quality multilevel scheme for partition-ing irregular graphs. SIAM J. Sci. Comput., 20(1):359-392, December 1998.

[10] Li Z, Zheng L, Zhang H. Solving pde models in modelica. 2008 Interna-tional Symposium on Information Science and Engieering. ISISE 08, 1:53-57, 2008.

[11] Ljubijankic' M, Nytsch-Geusen C. 3d/1d co-simulation von raumlufströ-mungen und einer luftheizung am beispiel eines thermischen modellhauses. Fourth GermanAustrian IBPSA Conference, BauSIM 2012, Berlin University of the Arts, 2012.

[12] Ljubijankic' M, Nytsch-Geusen C, Rädler J, Löffler M. Numerical coupling of modelica and cfd for building energy supply systems. 8th International Modelica Conference 2011, 2011.
[13] Engineering Mathematics and Computing Lab (EMCL) publication database., 2014 http://emcl.iwr.uni-heidelberg.de/79.html.

[14] Modelica and the Modelica Association. http://www.modelica.org.

[15] Modelica Association. Functional Mock-up Interface for Model Exchange and Co-Simulation, v. 2.0 beta 4 edition, 2012. https://www.fmi-standard.org/downloads [Accessed 2013-08-06].

[16] Preprint Series of Engineering Mathe-matics and Computing Lab (EMCL)., 2014. https://journals.ub.uniheidelberg.de/index.php/emcl-pp/issue/archive.

[17] Saldamli L. PDEModelica - A High- Level Language for Modeling with Partial Differential Equations. Dissertation 1016, Department of Computer and Informa-tion Science, Linköping University, 2006. http://urn.kb.se/resolve?urn=urn:nbn:se:liu:diva-7281.

[18] Saldamli L, Bachmann B, Wiesmann HJ, Fritzson P. A frame-work for describing and solving pde models in modelica. In Proceedings of the 4th International Modelica Conference, Hamburg, Ger-many, March 7-8, 2005.

[19] The HiFlow3 Multi-Purpose Finite Element Software. http://www.hiflow3.org.

[20] Stavåker K, Ronnås S, Wlotzka M, et al. Pde modeling with modelica via fmi im-port of hiflow $3 \mathrm{c}++$ components. Proceed-ings of SIMS 54th Conference, Bergen, Nor-way, 2013. 


\section{EUROSIM 2016 $9^{\text {th }}$ EUROSIM Congress on Modelling and Simulation}

City of Oulu, Finland, September 12 - 16, 2016
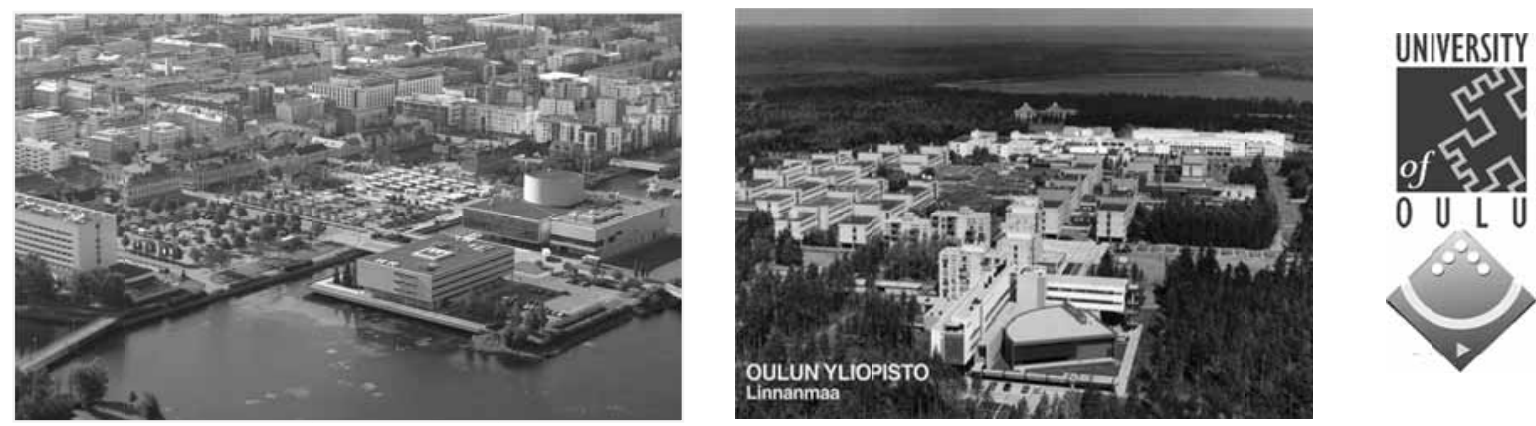

EUROSIM Congresses are the most important modelling and simulation events in Europe. For EUROSIM 2016, we are soliciting original submissions describing novel research and developments in the following (and related) areas of interest: Continuous, discrete (event) and hybrid modelling, simulation, identification and optimization approaches. Two basic contribution motivations are expected: M\&S Methods and Technologies and M\&S Applications. Contributions from both technical and non-technical areas are welcome.

Congress Topics The EUROSIM 2016 Congress will include invited talks, parallel, special and poster sessions, exhibition and versatile technical and social tours. The Congress topics of interest include, but are not limited to:

Intelligent Systems and Applications Hybrid and Soft Computing Data \& Semantic Mining Neural Networks, Fuzzy Systems \& Evolutionary Computation Image, Speech \& Signal Processing Systems Intelligence and Intelligence Systems Autonomous Systems Energy and Power Systems Mining and Metal Industry Forest Industry

Buildings and Construction Communication Systems

Circuits, Sensors and Devices

Security Modelling and Simulation
Bioinformatics, Medicine, Pharmacy and Bioengineering

Water and Wastewater Treatment, Sludge Management and Biogas Production

Condition monitoring, Mechatronics and maintenance

Automotive applications

e-Science and e-Systems

Industry, Business, Management, Human Factors and Social Issues

Virtual Reality, Visualization,

Computer Art and Games

Internet Modelling, Semantic Web and Ontologies

Computational Finance \& Economics
Simulation Methodologies and Tools Parallel and Distributed Architectures and Systems Operations Research Discrete Event Systems Manufacturing and Workflows Adaptive Dynamic Programming and Reinforcement Learning Mobile/Ad hoc wireless networks, mobicast, sensor placement, target tracking Control of Intelligent Systems Robotics, Cybernetics, Control Engineering, \& Manufacturing

Transport, Logistics, Harbour, Shipping and Marine Simulation

Congress Venue / Social Events The Congress will be held in the City of Oulu, Capital of Northern Scandinavia. The main venue and the exhibition site is the Oulu City Theatre in the city centre. Pre and Post Congress Tours include Arctic Circle, Santa Claus visits and hiking on the unique routes in Oulanka National Park.

Congress Team: The Congress is organised by SIMS - Scandinavian Simulation Society, FinSim - Finnish Simulation Forum, Finnish Society of Automation, and University of Oulu. Esko Juuso EUROSIM President, Erik Dahlquist SIMS President, Kauko Leiviskä EUROSIM 2016 Chair

Info: eurosim2016.automaatioseura.fi, office@automaatioseura.fi 


\title{
Probabilistic Drift Formulation of SIRS Models based on SPDEs and the Kolmogorov Equation
}

\author{
Günter Schneckenreither \\ Institute for Analysis and Scientific Computing, Vienna University of Technology, Wiedner Haupstraße 8-10, \\ 1040 Vienna, Austria; guenter.schneckenreither@tuwien.ac.at
}

Simulation Notes Europe SNE 25(3-4), 2015, 199-206

DOI: $10.11128 /$ sne. 25. tn. 10317

Received: October 5, 2015; Revised October 30, 2015;

Accepted: November 25, 2015

\begin{abstract}
The SIR (susceptible-infected-recovered) differential equations model for the spread of infectious diseases is very prominent in mathematical literature. The key interest of investigations is often to find and analyse extensions of the basic model structure in order to introduce a more detailed and supposedly realistic representation of the underlying disease and population dynamics. It is however true that problems and solutions in healthcare and health economics actually tend to require more and more sophisticated modelling approaches which are also capable of incorporating larger data sets as parametrisation in an effective way. This makes the comparison and combination of different modelling techniques and results an important research topic. This paper investigates a probabilistic drift formulation of the basic differential equations model which allows a very fine-grained parametrisation of the progression of diseases. It is shown that this formulation is capable of reproducing results from models with delay. Aggregation leads directly back to the traditional compartment approach and, in the heterogeneous case, a discrete representation can be interpreted as a system of local Markov processes. Furthermore some preliminary results on epidemiological measures like the basic reproduction number are presented.
\end{abstract}

\section{Introduction}

The SIR (susceptible-infected-recovered) epidemic model was originally derived by Kermack and McKendrick using a time-discrete scheme with multiple stages of disease progression [5]. For constant transition rates this model can be represented as a system of ordinary differential equations with three compartments, often referred to as the classical SIR(S) ODE model (1).

$$
\begin{aligned}
& \partial_{t} s(t)=-a s(t) i(t)+c r(t) \\
& \partial_{t} i(t)=a s(t) i(t)-b i(t) \\
& \partial_{t} r(t)=b i(t)-c r(t)
\end{aligned}
$$

Among the basic principles of the epidemic model the following are key to this paper:

(D1) The rates $b$ and $c$ and as well as the force of infection $f:=a i(t)$ determine the (linear) flow or transition rates between the three compartments.

(D2) Since the force of infection acts on the number of susceptible individuals in a linear fashion, the incidence rate $a i(t) s(t)$ as a bilinear function implies a homogeneous mixing of the population.

(D3) For the basic SIRS model, the epidemic threshold (basic reproduction number) is given by $R_{0}=\frac{a}{b}$. This threshold controls the existence of an endemic equilibrium.

It is well known that complex dynamic patterns such as bifurcations or periodic behaviour are more likely to occur when the spread of infection is constrained by heterogeneity or delay $[1,4,10,11]$.

Nevertheless, as a fist step towards a system description which is capable of simulating such behaviour, we regard the following one-dimensional SIS simplification $(S=R)$.

$$
d \xi(t)=-b \xi(t) d t+a(1-\xi(t)) \xi(t) d t
$$

The state space of this dynamical system is the continuous bounded disease space $\Xi:=[0,1]$, where a disease state $\xi \in \Xi$ indicates the strength or concentration of an infectious disease in a population, $\xi=1$ meaning fully infected. 
In order to obtain a discrete signal from this model define a susceptible domain $\Xi_{s}:=\left[0, \frac{1}{2}\right)$ and a infected domain $\Xi_{i}:=\left[\frac{1}{2}, 1\right]$ such that $\Xi=\Xi_{s} \cup \Xi_{i}$.

In this paper we extend the one-dimensional SIS model (2) with the periodic disease space $\Xi:=[0,1)_{\text {per }}$ and define instead of the susceptible and infected regions, the domains susceptible, infected, exposed, contagious and recovered/immune, $\Xi_{s}, \Xi_{i}, \Xi_{e}, \Xi_{c}, \Xi_{r}$, such that

$$
\Xi=\Xi_{s} \dot{\cup} \Xi_{i} \dot{\cup} \Xi_{r}, \quad \Xi_{e} \dot{\cup} \Xi_{c} \subseteq \Xi_{i} .
$$

It is obvious that the resulting disease space leads to a model for SEIRS-type epidemics (see Figure 1). The
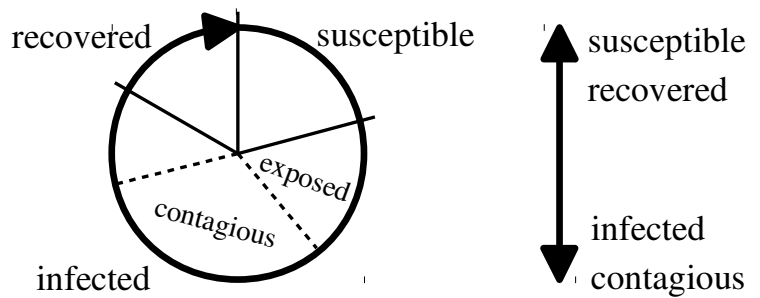

Figure 1: Periodic disease space (SIRS) compared to bounded disease space (SIS).

basic structure of the equations investigated in this paper is

$$
d \xi(t)=g_{1}(\xi(t)) d t+g_{2}(\xi(t)) f(\xi(t)) d t
$$

Using a probabilistic approach (Kolmogorov forward equation) [1] and nonlinear incidence rates [4] it can be shown that the resulting transport (and diffusion) formulation (4) is a generalisation of the classical compartment approach and also of delay models.

For completeness this paper also introduces an arbitrary heterogeneity space $X$ and takes into account random additive (white) noise such that theoretically we finally arrive at a stochastic partial differential equation (SPDE). For simplicity and readability most equations are written without the noise or respective diffusion term. For noise in the bounded domain $\Xi=[0,1]$ we have to ensure that the boundaries of the domain are honoured (compare [11]).

\section{Heterogeneity States}

The introduction of heterogeneity states in epidemic models has a long tradition and leads to abstract Cauchy problems and parabolic PDEs [8] with richer patterns of behaviour. From a modelling point of view, heterogeneity allows to simulate variable rates and parameters (i.e. heterogeneous populations) and to obtain inhomogeneous propagation and spread of a disease.

Let $X$ be a finite dimensional topological vectorspace such that $\xi(t, x)$ indicates the abundance of a infectious disease (think of viral concentration, number of infected individuals, ...) with heterogeneity state $x \in X$. The topological vectorspace $X$ can for example represent a spatial domain or the ages of the individuals of a population. The most natural idea for epidemic models with heterogeneous populations is that the force of infection depends on an aggregated (nonlocal) concentration of the disease $\xi_{N}(t, x)$ instead of the local state $\xi(t, x)$.

The aggregated state can for example be a weighted integral

$$
\xi_{N}(t, x):=\int_{X} \lambda(x, y) \xi(t, y) d y
$$

with kernel $\lambda(x, y)$, which allows to model different types of nonlocal interaction [9]. For example a Gaussian kernel can simulate a diffusive interaction process and the Taylor series expansion of $\xi(t, y)$ around $x$ allows to find a differential representation $\xi_{N}=\lambda_{1} \xi+$ $\lambda_{2} \Delta_{x} \xi$.

Technically the aggregated state $\xi_{N}(t, x)$ replaces the actual local state $\xi(t, x)$ in the argument of the force of infection $f(\cdot)$.

The epidemic threshold for heterogeneous SIS systems [11] corresponds under some assumptions (separable and symmetric kernel $\lambda$, constant population density) to the integral

$$
R_{0}=\int_{X} \lambda(x, x) \frac{a(x)}{b(x)} d x
$$

\section{Probabilistic Formulation and Semilinear Incidence}

A probabilistic formulation of homogeneous SIR models can be found in [1] for example, where the authors transform the homogeneous SIR model into a transport problem and derive the Kolmogorov forward equation from a discrete SIR approach. They also state that the probabilistic representation with no diffusion has degenerate distributions as its limit, conforming with the theory on epidemic thresholds and equilibria. 


\subsection{SIS Model}

For the heterogeneous SIS model (nonlocal force of infection) the stochastic formulation

$$
\begin{aligned}
d \xi(t, x)= & -b \xi(t, x) d t \\
& +a(1-\xi(t, x)) \xi_{N}(t, x) d t
\end{aligned}
$$

is (locally) a Itô diffusion since the drift term is Lipschitz continuous and the corresponding Kolmogorov forward equation [7] (probabilistic approach) is

$$
\begin{aligned}
& \frac{\partial}{\partial t} p(t, x, \xi)=\left(a \xi_{N}+b\right) p(t, x, \xi)+ \\
& +\left(\left(a \xi_{N}+b\right) \xi-a \xi_{N}\right) \frac{\partial}{\partial \xi} p(t, x, \xi) .
\end{aligned}
$$

The drift term can be separated into

$$
\begin{aligned}
& g_{1}(t, x, \xi):=-b \xi \\
& g_{2}(t, x, \xi):=a(1-\xi) \\
& f\left(t, x, \xi_{N}\right):=\xi_{N}
\end{aligned}
$$

such that $g_{1} \leq 0$ defines a constant drift towards the susceptible state $\xi=0$ and, depending on the force of infection $f\left(\xi_{N}\right), g_{2} \geq 0$ generates drift towards the infected state $\xi=1$. Note that all three functions are linear in $\xi$ or $\xi_{N}$ respectively (see Figure 2). In the

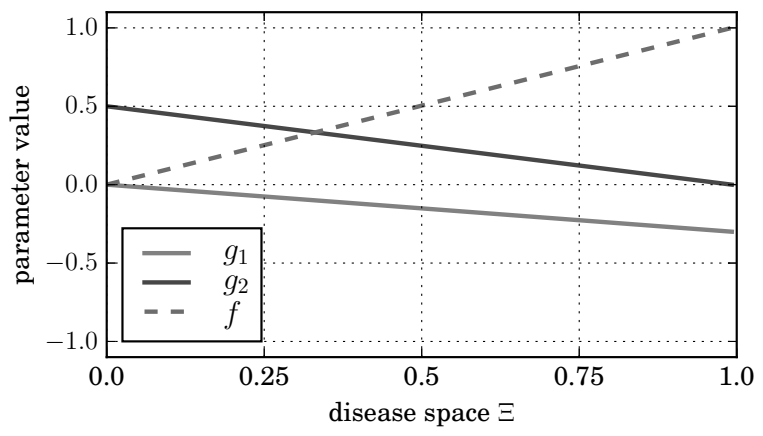

Figure 2: Parameter functions of the SIS model for arbitrary $a$ and $b$.

stochastic formulation (7) $\xi(t, x)$ is actually a random field. In the probabilistic representation (8) it is necessary to replace the aggregated random variable $\xi_{N}$ respectively the force of infection $f\left(\xi_{N}\right)$ with a statistic [8]. A plausible choice is of course the expectation value

$$
\mathbb{E}\left[f\left(\xi_{N}\right)\right]=\int_{\Xi} f(\eta) p_{N}(t, x, \eta) d \eta
$$

where $p_{N}(t, x, \xi)=\int_{X} \lambda(x, y) p(t, y, \xi) d y$ is the distribution of the accumulated random variable $\xi_{N}$.

\subsection{SEIRS Model}

For the stochastic SEIRS approach, i.e. when the disease space is periodic, and the corresponding Kolmogorov forward equation

$$
\begin{array}{r}
\frac{\partial}{\partial t} p(t, x, \xi)=-\frac{\partial}{\partial \xi}\left\{\left(g_{1}(\xi)+\right.\right. \\
\left.\left.+g_{2}(\xi) \int_{\Xi} f(\eta) p_{N}(t, x, \eta) d \eta\right) p(t, x, \xi)\right\}
\end{array}
$$

drift happens in positive direction only (except maybe for local $\Xi$ areas with negative drift).

Let $g_{1}$ define a constant drift, which describes normal disease progression like entering and leaving a contagious phase $\Xi_{c}$, which is a subset of the infected phase $\Xi_{i}$, or transition from infected to recovered/immune $\Xi_{r}$ and from recovered to susceptible $\Xi_{s}$. The drift from the susceptible domain to the infected domain however shall be generated by $g_{2}$ with strength controlled by the force of infection $f$. From a certain point of view, the function $g_{2}$ compensates for the lack of drift generated by $g_{1}$ in the susceptible domain $\Xi_{s}$. The total drift is given by

$$
g(\xi):=g_{1}(\xi)+g_{2}(\xi) \int_{\Xi} f(\eta) p_{N}(t, x, \eta) d \eta .
$$

Figure 3 shows a possible configuration of the parameter functions.

We make the following heuristic (and not fully necessary) but plausible assumptions on the parameter functions.

(P1) The basic drift term $g_{1}$ is mostly constant with a value $k \in[0,1]$ except for a region around the interface between the susceptible and the infected domain, where $g_{1}$ vanishes. The value $k$ determines the speed of disease progression. Of course the exact shape of $g_{1}$ is a result of modelling decisions. Also the value $k$ is actually only a scaling variable which relates disease progression to time (see section 3). 


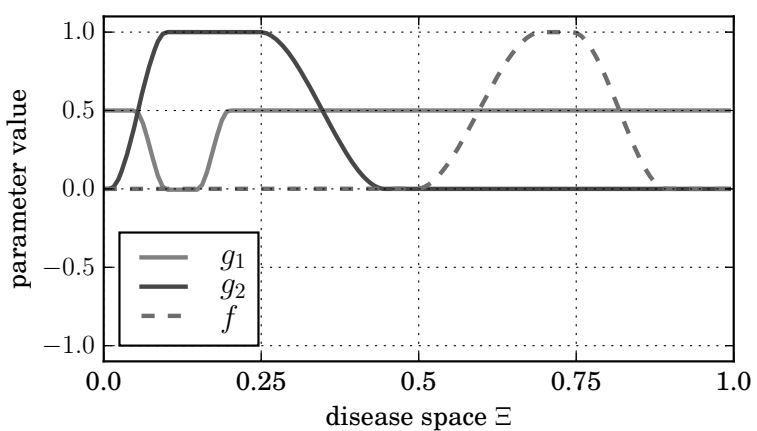

Figure 3: Parameter functions of the SIRS model with periodic disease space. Here $g_{1}$ actually takes (small) negative values in the susceptible domain and $g_{2}$ takes very large values compared to $g_{1}$.

(P2) The incidence drift term $g_{2}$ is larger than zero only in a region around the interface between the susceptible and the infected domain.

(P3) As a consequence in the infected region the total drift term is mostly constant $\left.g\right|_{\Xi_{i}} \equiv k$ independent of the shape of $p_{N}$ (i.e. the force of infection).

(P4) The function $f$ is larger than 0 only in the contagious domain or in other words, the support of $f$ determines the contagious domain (compare fuzzy sets). We can assume that $f$ is normalised $\|f\|_{L^{\infty}(\Xi)}=\|f\|_{\infty}=1$.

(P5) There exists a configuration $p_{i}$ of $p_{N}$ such that the force of infection takes a maximum value.

Set $F^{-1}:=\|f\|_{1}$ then this configuration is given by $p_{i}(\xi)=F f(\xi)$ and the inequality

$$
1=F\|f\|_{1} \leq F\|f\|_{2} \leq F\|f\|_{\infty}=F
$$

shows that $F^{-1} \leq\|f\|_{2} \leq 1$.

We can interpret this configuration as a situation in which locally all susceptible individuals come into contact with infected individuals and the speed of spread is at the maximum. In other words $p_{i}$ is fully infectious.

Note that these assumptions restrict the drift SIRS model to a small subset of possible configurations. They however simplify some technical considerations in the following sections. But still the probabilistic formulation is capable of producing patterns that cannot be obtained with the stochastic formulation (see Figure 6).

\section{Delay Models}

As mentioned before delay plays an important role in the dynamics of epidemiology. In [4] three different types of delays are distinguished:

- Temporary immunity corresponds to the recovered/immune phase after the infection period.

- Delay caused by the latency in a vector happens when infection is spread by agents (e.g. mosquitoes).

- The latent period in a host is the time delay between infection and the contagious phase.

The following delay differential equation

$$
d \xi(t)=-b \xi(t) d t+a(1-\xi(t)) \xi(t-\tau) d t
$$

models a latent period in the host [4]. The corresponding Kolmogorov forward equation is

$$
\frac{\partial}{\partial t} p(t, \xi)=-\frac{\partial}{\partial \xi}\left\{\left(-b \xi+a(1-\xi) \xi_{T}\right) p(t, \xi)\right\}
$$

where again we replace the stochastic force of infection $f\left(\xi_{T}\right)$ with the expectation $\mathbb{E}[f(\xi(t-\tau))]$. Since in (P4) it was assumed that the support of the force of infection $f$ is $\Xi_{c}$, the expectation can be written as

$$
\mathbb{E}[f(\xi(t-\tau))]=\int_{\Xi_{c}} f(\eta) p(t-\tau, \eta) d \eta .
$$

From (P3) it follows that drift (velocity) is constant $g(\xi) \equiv k$ in $\Xi_{i}$ and especially in a region around $\Xi_{c}$ such that

$$
\left.\frac{\partial}{\partial t} p(t, \xi)\right|_{\Xi_{i}}=-\left.k \frac{\partial}{\partial \xi} p(t, \xi)\right|_{\Xi_{i}}
$$

which by the formal argument with scaling $\xi=k \tau$

$$
\lim _{\tau \rightarrow 0} \frac{p(t, \xi)-p(t-\tau, \xi)}{\tau}=\lim _{\tau \rightarrow 0} \frac{k p(t, \xi+k \tau)-k p(t, \xi)}{k \tau}
$$

leads to

$$
p(t-\tau, \xi)=p(t, \xi+k \tau)
$$


in $\Xi_{i}$. Inserting into (18) results in

$$
\begin{aligned}
\mathbb{E}[f(\xi(t-\tau))] & =\int_{\Xi_{c}} f(\eta) p(t, \eta+k \tau) d \eta \\
& =\int_{\Xi_{c}+k \tau} f(\eta-k \tau) p(t, \eta) d \eta
\end{aligned}
$$

Setting $\Xi_{c^{\prime}}:=\Xi_{c}+k \tau \subset \Xi_{i}$ finally means that the new contagious domain was shifted to the right by the scaled time delay $k \tau$ and we arrive back at the original model with different (shifted) force of infection $f(\xi)$.

This shows that the probabilistic approach is very suitable for modelling delay in epidemic spread. Or in other words, the probabilistic formulation is inherently delayed.

\section{Discretisation and Numerical Scheme}

For the sake of regularisation [2, 3] a relatively small noise respectively diffusion term is added to the equations. As a numerical scheme for the stochastic equations the straight-forward explicit Euler-Maruyama method [6] is used.

\subsection{Discretisation of the Probabilistic Formulation}

For the probabilistic formulations (Kolmogorov equations), a local Markov chain [8] representation can be constructed.

To that end let $\mathbf{p}(t, x) \in \mathbb{R}_{+}^{n}$ be a discretisation of $p(t, x, \xi)$ in the disease space $\Xi$ with $\sum_{i=1}^{n} p_{i}(t, x)=1$ for all $t$ and $x$. The local Kolmogorov equation with discretised disease space is formally given by

$$
\frac{\partial}{\partial t} \mathbf{p}=-\frac{\partial}{\partial \xi}\{\mathbf{g} \odot \mathbf{p}\}
$$

where $\odot$ denotes the element-wise multiplication and $\mathbf{g}$ is the vectorised drift term. From (13) we conclude that

$$
\mathbf{g}=\mathbf{g}_{1}+\mathbf{g}_{2}\left(\mathbf{f} \cdot \mathbf{p}_{N}\right)
$$

where $\mathbf{f} \cdot \mathbf{p}_{N}$ is the scalar product. The accumulated discrete distribution $\mathbf{p}_{N}$ is calculated from a finite number of discretised heterogeneity states from $X$.
Define the differentiation matrices

$$
D_{+}=\left(\begin{array}{cccc}
-1 & & & +1 \\
+1 & -1 & & \\
& +1 & -1 & \\
& & +1 & -1
\end{array}\right), \quad D_{-}=\left(\begin{array}{rrrr}
-1 & +1 & & \\
& -1 & +1 & \\
& & -1 & +1 \\
& & & -1
\end{array}\right),
$$

which calculate the derivative in negative and positive $\xi$ direction respectively (up to discretisation length and here displayed for the periodic case only), then

$$
\begin{aligned}
\frac{\partial}{\partial t} \mathbf{p} & =D_{+}(\mathbf{g} \odot \mathbf{p})_{>0}+D_{-}(\mathbf{g} \odot \mathbf{p})_{<0} \\
& =\left(D_{+} \operatorname{diag}\left(\mathbf{g}_{>0}\right)+D_{-} \operatorname{diag}\left(\mathbf{g}_{<0}\right)\right) \mathbf{p} \\
& =M(\mathbf{g}) \mathbf{p},
\end{aligned}
$$

where $\operatorname{diag}(\mathbf{a})$ is the diagonal matrix with the vector $\mathbf{a}$ as its diagonal.

Accordingly the SIRS model with discretised disease space, without diffusion and parameters as in Figure 3 locally corresponds to the Markov model visualised in Figure 4 with transition matrix $I+d t M(\mathbf{g})$ portrayed in Figure 5 and also to a system of ODEs with a finite number of compartments.

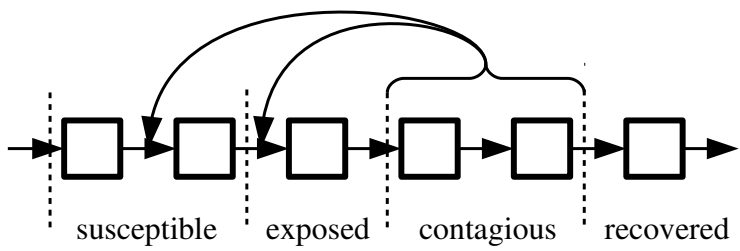

Figure 4: Discretised periodic disease space (SIRS) with feedback (concentration in $\Xi_{c}$ controls force of infection which determines the flow from $\Xi_{s}$ to $\Xi_{i}$ ).

\subsection{Global Explicit Scheme}

The corresponding global explicit iteration scheme to (25)-(27) can be formulated as a cellular automaton [8]. The discretisation of the heterogeneity space $X$ can be interpreted as a lattice of cells, which leads to a formal description of a cellular automaton with

(X) a discretisation of $X$ as cellular space,

(S) vectors $\mathbf{p} \in \mathbb{R}_{+}^{n}$ as cell states,

(N) a finite sum (discretisation of (5)) as a discrete version of the accumulated (neighbourhood-) state $\mathbf{p}_{N}$,

(L) and a local iteration rule given by (25)-(27). 


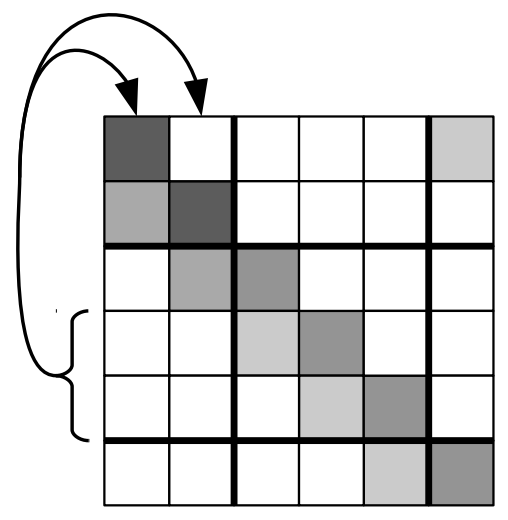

Figure 5: Basic structure of the transition matrix. Concentration in the contagious domain controls flow from the susceptible to the infected domain. For simplicity no negative flow is shown.

In a more abstract fashion also the stochastic formulation can be interpreted in the context of cellular automata.

(S) Instead of discretised distributions (vectors), the states of the cells shall be random variables in $\Xi$.

(N) Accordingly the accumulated random state is a multivariate random variable or defined by a measurable function $\Xi^{d} \rightarrow \Xi$, which maps "neighbouring" random variables onto a "accumulated" random variable.

(L) The local iteration rule is defined by a conditional probability or Markov kernel.

From a modelling point of view, the cellular automaton approach can be classified as a direct modelling approach (local Markov model) with discrete heterogeneity space, whereas the discretisation of the differential equation formulations is a numerical scheme. Visualisations of a simulation run can be seen in Figure 6 .

\section{The Probabilistic Formulation Extends The Compartment Approach}

For the SIRS model let the dimension of the discretisation of the disease space be $n:=3$ and identify the first dimension with $\Xi_{s}$, the second dimension with $\Xi_{i}=\Xi_{c}$ and the third dimension with $\Xi_{r}$. For the discretisation
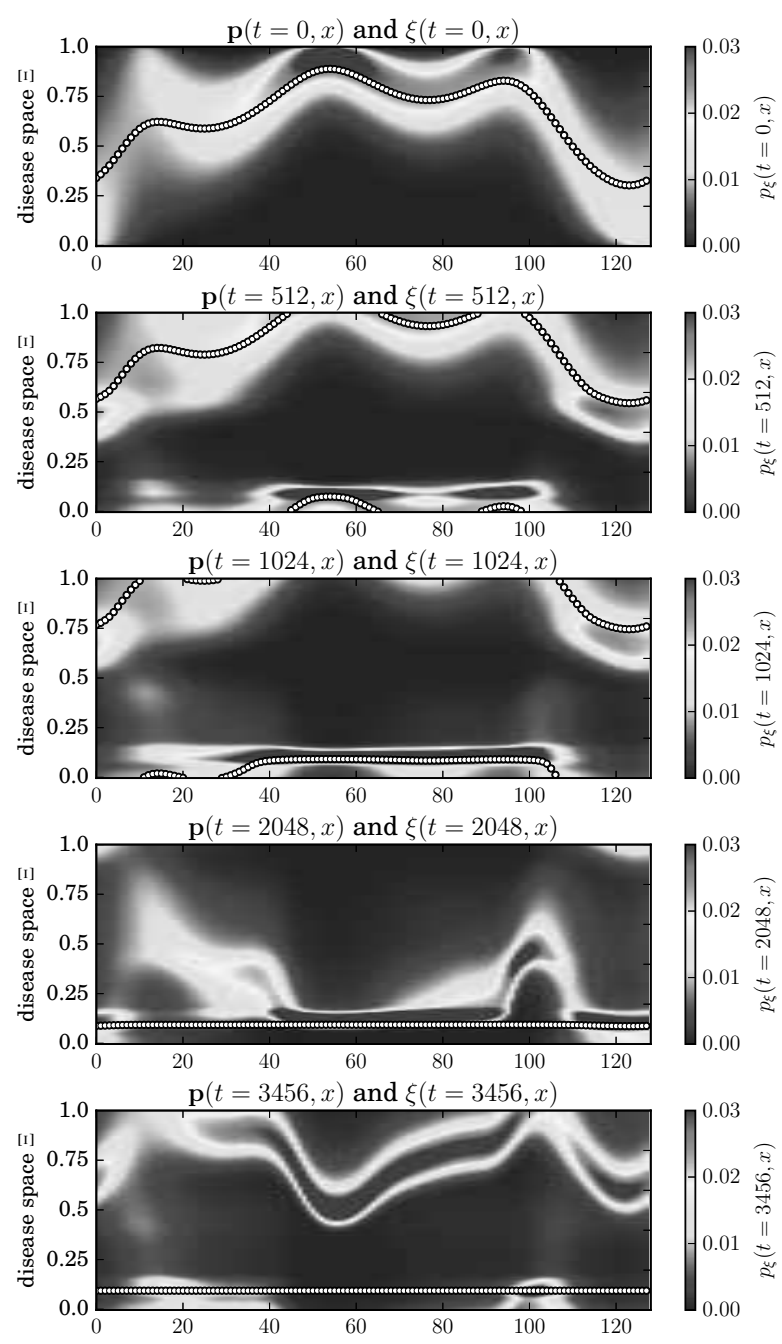

Figure 6: Visualisation of the probabilistic heterogeneous SIRS model $(\mathbf{p}(t, x, \boldsymbol{\xi})$, colorscale) and the corresponding stochastic model $(\xi(t, x)$, black/white line) without diffusion. The (periodic) heterogeneity space $X$ is displayed in horizontal direction.

of the parameter functions $g_{1}, g_{2}$ and $f$ we conclude the following from (P1)-(P4).

$$
\begin{aligned}
0 \approx g_{11} & :=\int_{\Xi_{s}} g_{1}(\xi) d \xi \\
b & :=g_{12}:=\int_{\Xi_{i}} g_{1}(\xi) d \xi \\
c & :=g_{13}:=\int_{\Xi_{r}} g_{1}(\xi) d \xi \\
a & :=g_{21}:=\int_{\Xi_{s}} g_{2}(\xi) d \xi
\end{aligned}
$$




$$
\begin{aligned}
0 \approx g_{22} & :=\int_{\Xi_{i}} g_{2}(\xi) d \xi \\
0 \approx g_{23} & :=\int_{\Xi_{r}} g_{2}(\xi) d \xi \\
0 \approx f_{1} & :=\int_{\Xi_{s}} F f(\xi) d \xi \\
1 \approx f_{2} & :=\int_{\Xi_{i}} F f(\xi) d \xi \\
0 \approx f_{3} & :=\int_{\Xi_{r}} F f(\xi) d \xi
\end{aligned}
$$

Instead of discretisation by integration as shown above, it should also be possible to use point evaluations at the interfaces between the different subdomains. According to (P4), in (34)-(36) also the $L^{\infty}$-norm could be used.

Inserting in the evolution equation (25)-(27) leads to

$$
\begin{aligned}
& \frac{\partial}{\partial t}\left(\begin{array}{l}
p_{1} \\
p_{2} \\
p_{3}
\end{array}\right)=D_{+} \operatorname{diag}\left(a p_{N, 2}, b, c\right)\left(\begin{array}{l}
p_{1} \\
p_{2} \\
p_{3}
\end{array}\right) \\
& =\left(\begin{array}{ll}
-a p_{N, 2} & +c \\
+a p_{N, 2} & -b \\
& +b-c
\end{array}\right)\left(\begin{array}{l}
p_{1} \\
p_{2} \\
p_{3}
\end{array}\right) .
\end{aligned}
$$

This corresponds to the compartment formulation of the heterogeneous SIRS model. Additionally setting $\mathbf{p}_{N}:=\mathbf{p}$ results in the classical homogeneous SIRS ODE model (1).

\section{Outlook - Basic Reproduction Number}

The basic reproduction number $R_{0}$ can be interpreted as the number of secondary infections created by one infectious individual in a fully susceptible population $[11,1,10,4]$.

In order to find a similar measure for the probabilistic drift formulation we start from a fully susceptible homogeneous population which can be represented by the equilibrium distribution $p_{s}$ of (13) when starting from distributions with support in $\Xi_{s}$ or an arbitrary nonequilibrium distribution $p_{s}$ with support in $\Xi_{s}$. There also exists a configuration $p_{k}$ with unit force of infection, which is characterised by

$$
g(\xi)=g_{1}(\xi)+g_{2}(\xi) \int_{\Xi} f(\eta) p_{k}(\eta) d \eta \approx k .
$$

For example using (P5) and $p_{s}$ we can set

$$
p_{k}:=\left(1-\frac{k}{\max g_{2}}\right) p_{s}+\frac{k}{\max g_{2}} p_{i} .
$$

\subsection{Ratio of Infectiousness}

If we set $p_{N}:=p_{k}$ and assume that the force of infection stays constant over time, (13) can be written as

$$
\frac{\partial}{\partial t} p(t, \xi)=-k \frac{\partial}{\partial \xi} p(t, \xi) .
$$

Let us calculate the infectiousness of the solution of the initial value problem (40) with initial condition $p(0, \xi)=p_{k}(\xi)$ at some later time $t$ and compare it with the infectiousness of $p_{k}$. From (21) we know that $p(t, \xi)=p(0, \xi-k t)=p_{k}(\xi-k t)$.

Accordingly the function $Q_{1}:[0,1)_{\text {per }} \rightarrow \mathbb{R}$,

$$
\begin{aligned}
Q_{1}(t) & :=\frac{\exp \left(\int p_{k}(\xi-k t) f(\xi) d \xi\right)}{\exp \left(\int p_{k}(\xi) f(\xi) d \xi\right)} \\
& =\frac{\exp \left(\mathbb{E}^{p_{k}}[f(\xi+k t)]\right)}{\exp \left(\mathbb{E}^{p_{k}}[f(\xi)]\right)}
\end{aligned}
$$

can be used for measuring the delayed $(t)$ infectiousness of an initially susceptible population with one unit force of infection and stores information about the strength and succession of epidemic waves. Due to the simplifications made, this measure may not be very accurate in practice.

\subsection{Balance of Infectiousness}

From (29) and (31) we may assume that

$$
R_{0}:=\frac{\int_{\Xi_{s}} g_{2}(\xi) d \xi}{\int_{\Xi_{c}} g_{1}(\xi) d \xi}
$$

is useful as a measure for secondary infections. This is motivated by the definition of the basic reproduction number of the compartment SIRS model, which is the ratio between the flow rate to the contagious domain $a$ and the flow rate from the contagious domain $b$ under the condition of a fully susceptible population with one unit force of infection.

In- and outflow of the contagious domain under the same conditions in the probabilistic model at time $t$ is accessible through the balance equation

$$
\begin{aligned}
\frac{\partial}{\partial t} \int_{\Xi_{c}} p(t, \xi) d \xi & =-k \int_{\Xi_{c}} \frac{\partial}{\partial \xi} p(t, \xi) d \xi \\
& =k\left(p\left(t, \xi_{0}\right)-p\left(t, \xi_{1}\right)\right)
\end{aligned}
$$

where $\xi_{0}$ and $\xi_{1}$ are the boundaries of $\Xi_{c}$. But since the contagious domain is defined by $f$, with integration by 
parts we arrive at

$$
\begin{aligned}
& \frac{\partial}{\partial t} \int_{\Xi} p(t, \xi) f(\xi) d \xi=-k \int_{\Xi} f(\xi) \frac{\partial}{\partial \xi} p(t, \xi) d \xi \\
& =k \int_{\Xi} p(0, \xi) f^{\prime}(\xi+k t) d \xi=k \mathbb{E}^{p_{k}}\left[f^{\prime}(\xi+k t)\right]
\end{aligned}
$$

which yields

$$
Q_{2}(t):=\frac{\exp \left(k p\left(t, \xi_{0}\right)\right)}{\exp \left(k p\left(t, \xi_{1}\right)\right)}=\frac{\exp \left(k p_{k}\left(\xi_{0}-k t\right)\right)}{\exp \left(k p_{k}\left(\xi_{1}-k t\right)\right)}
$$

and

$$
Q_{3}(t):=\exp \left(k \mathbb{E}^{p_{k}}\left[f^{\prime}(\xi+k t)\right]\right)
$$

as possible time-dependent measures for secondary infections. As before we must note that these measures rely on a great number of simplifications.

For the extremely simplified case

$$
\begin{aligned}
g_{1}(\xi) & :=\mathbb{I}_{\left[\frac{0}{4}, \frac{1}{4}\right)}(\xi) \\
g_{2}(\xi) & :=k \mathbb{I}_{\left[\frac{1}{4}, \frac{4}{4}\right)}(\xi) \\
f(\xi) & :=\mathbb{I}_{\left[\frac{2}{4}, \frac{3}{4}\right)}(\xi) \\
p_{k}(\xi) & :=4(1-k) \mathbb{I}_{\left[\frac{0}{4}, \frac{1}{4}\right)}(\xi)+4 k \mathbb{I}_{\left[\frac{2}{4}, \frac{3}{4}\right)}(\xi)
\end{aligned}
$$

we obtain (partially in a formal way only) $R_{0}=\frac{1}{k}$ and the functions $Q_{1}, Q_{2}, Q_{3}$ portrayed in Figure 7. In a next step numerical tests must be conducted in order to find information about the reliability of these measures.
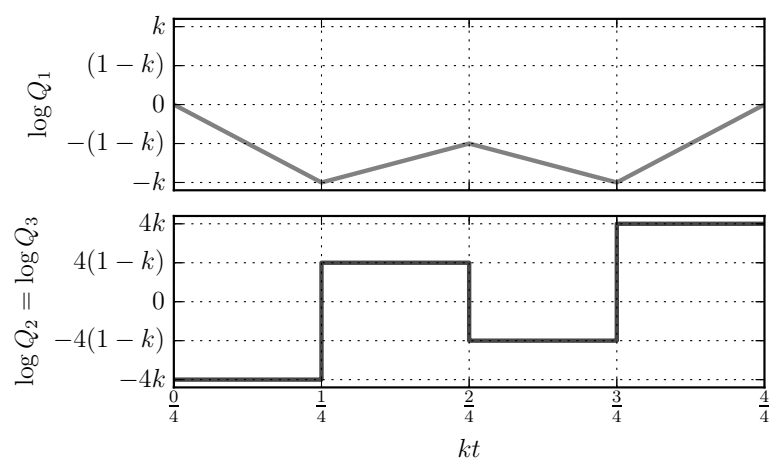

Figure 7: With the parameter functions defined in (47)-(50) the functions $Q_{2}$ and $Q_{3}$ coincide in a formal way. In logarithmic scale the flow-based measure $Q_{2}=Q_{3}$ is the derivative of the delay-based measure $Q_{1}$. This figure assumes $k \approx \frac{2}{3}$.

\section{Acknowledgements}

This work was funded by the K-Project DEXHELPP, which is supported by Austrian BMVIT and BMWFW as well as the state of Vienna via COMET - Competence Centers for Excellent Technologies. The COMET program is processed by FFG.

\section{References}

[1] Chalub FAAC, Souza MO. The SIR epidemic model from a PDE point of view. Mathematical and Computer Modelling. 2011; 53(7-8): 1568-1574. doi: 10.1016/j.mcm.2010.05.036.

[2] Gyöngy I. Existence and uniqueness results for semilinear stochastic partial differential equations. Stochastic Processes and their Applications. 1998; 73(2): 271299.

[3] Gyöngy I, Nualart D. Implicit scheme for quasi-linear parabolic partial differential equations perturbed by space-time white noise. Stochastic Processes and their Applications. 1995; 58(1): 57-72.

[4] Huang G, Takeuchi Y. Global analysis on delay epidemiological dynamic models with nonlinear incidence. Journal of Mathematical Biology. 2010; 63(1): 125-139. doi: 10.1007/s00285-010-0368-2.

[5] Kermack WO, McKendrick AG. A Contribution to the Mathematical Theory of Epidemics. Proceedings of the Royal Society A: Mathematical, Physical and Engineering Sciences. 1927; 115(772): 700-721.

[6] Lord G, Powell CE, Shardlow T. An introduction to computational stochastic PDEs. Cambridge University Press; 2014.

[7] Øksendal BK. Stochastic differential equations; an introduction with applications. Berlin: Springer; 2007.

[8] Schneckenreither G. Developing Mathematical Formalisms for Cellular Automata in Modelling and Simulation [master thesis]. Vienna University of Technology; 2014.

[9] Schneckenreither G. Modelling SIR-type epidemics by ODEs, PDEs, difference equations and cellular automata - A comparative study. Simulation Modelling Practice and Theory. 2008; 16(8): 1014-1023, doi: 10.1016/j.simpat.2008.05.015.

[10] Vynnycky E, White RG. An introduction to infectious disease modelling. Oxford University Press; 2010.

[11] Widder A, Kuehn C. Heterogeneous Population Dynamics and Scaling Laws near Epidemic Outbreaks. ArXiv e-prints. 2014; 1411.7323. 


\title{
ARGESI M Benchmark C13 'Crane and Embedded Control' with SI MULI NK - modelled Dynamics and MATLAB-programmed Control
}

\author{
Georg Janisch, Miriam Leopoldseder*, Andreas Körner
}

Institute for Analysis and Scientific Computing, Vienna Univ. of Technology Wiedner Hauptstraße 8 1040 Vienna, Austria; miriam.leopoldseder@tuwien.ac.at

Simulation Notes Europe SNE 25(3-4), 207 - 209

DOI: $10.11128 /$ sne.25.bn13.10318

Received: February 20, 2015; Revised September 20, 2015;

Accepted: October 10, 2015;

Abstract. ARGESIM Benchmark C13 'Crane and Embedded Control' is based on nonlinear and a linear continuous dynamics for a crane crab and on discrete control by a linear observer model. This solution makes use of the M ATLAB/Simulink system in a 'mixed' manner. The continuous dynamics are modelled graphically in the Simulink environment - using partly connected blocks and partly MATLAB functions. The discrete control with observer model and the diagnosis is programmed in MATLAB, framed by a sampled date discrete submodel in Simulink connected to the continuous submodels for the dynamics. This approach allows fast modelling, but deeper insight into the simulation environment is necessary.

\section{I ntroduction}

MATLAB is a high-level language and interactive environment for numerical computation, visualization, and programming [1]. It is based on numerical vector and matrix manipulation. SIMULINK is a graphical block diagram environment for multidomain simulation and model-based design [2]. Within Simulink, MATLAB functions can be used, with high complexity. This solution makes use of the MATLAB/Simulink system in a 'mixed' manner.

The continuous dynamics are modelled graphically in the Simulink environment - using partly connected blocks and partly MATLAB functions. The discrete control with observer model and the diagnosis is programmed in MATLAB, framed by a sampled date discrete submodel in Simulink connected to the continuous submodels for the dynamics.

\section{Modelling}

Basis for the dynamic model are the linear and nonlinear mechanical equations for the crane crab, and the 'electrical equation for the DC motor

Linear model for crane crab:

$$
\begin{gathered}
\ddot{x}_{c}=\frac{f_{c}}{m_{c}}+g \frac{m_{l}}{m_{c}} \alpha-\frac{d_{c}}{m_{c}} \dot{x}_{c} \\
r \ddot{\alpha}=-g\left(1+\frac{m_{l}}{m_{c}}\right) \alpha+\left(\frac{d_{c}}{m_{c}}-\frac{d_{l}}{m_{l}}\right) \dot{x}_{c}-r \frac{d_{l}}{m_{l}} \dot{\alpha} \\
-\frac{f_{c}}{m_{c}}+\frac{f_{d}}{m_{l}} \\
x_{l}=x_{c}+r \alpha
\end{gathered}
$$

Nonlinear model for crane crab:

$\ddot{x}_{c}\left[m_{c}+m_{l} \sin \alpha^{2}\right]=-d_{c} \dot{x}+f_{c}+f_{d} \sin \alpha^{2}+$ $m_{l} \sin \alpha\left[r \dot{\alpha}^{2}+g \cos \alpha\right]-d_{l} \dot{x} \sin \alpha^{2}$

$\ddot{\alpha} r^{2}\left[m_{l} \sin \alpha^{2}+m_{c}\right]=\left[f_{d} \frac{m_{c}}{m_{l}}-f_{c}+\right.$

$\left.d_{c} \dot{x}\right] r \cos \alpha-\left[g\left(m_{l}+m_{c}\right)+\right.$

$\left.m_{l} r \dot{\alpha}^{2} \cos \alpha\right] r \sin \alpha-d_{l}\left[\frac{m_{c}}{m_{l}}\left(\dot{x} r \cos \alpha+r^{2} \dot{\alpha}\right)+\right.$

$\left.r^{2} \dot{\alpha} \sin \alpha^{2}\right]$

$x_{l}=x_{c}+r \sin \alpha$

Linear model for DC motor:

$t_{m} \dot{f}_{c}+f_{c}=k_{m} v$

In general, for dynamic model parts Simulink submodels with continuous blocks were used. For discrete model parts - i.e. the controller and the diagnosis - Simulink submodel(s) with MATLAB functions inside were used (Figure 1 - modelling overview) 


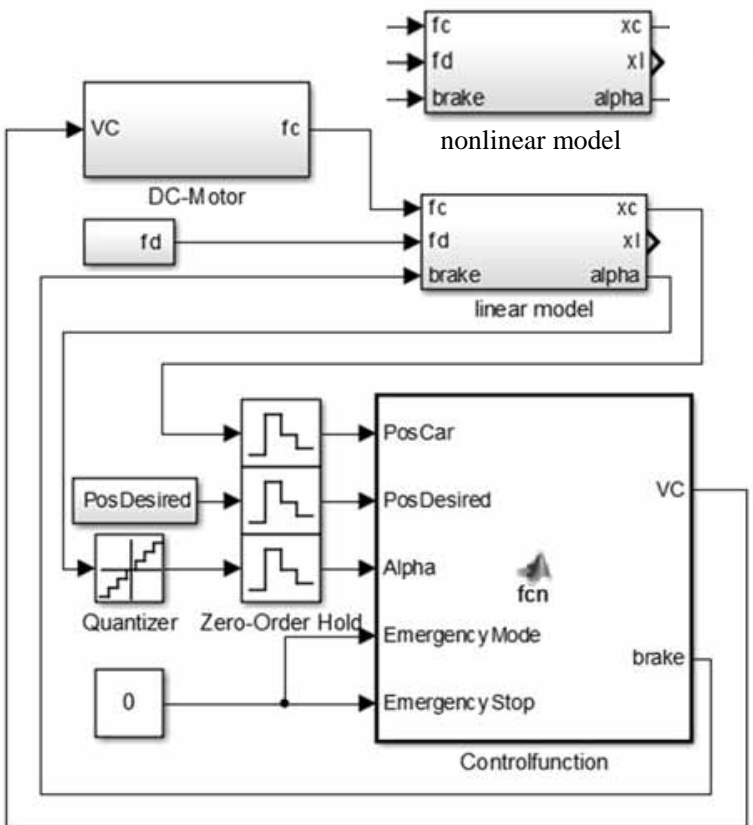

Figure 1. Overall model with continuous dynamics (linear / nonlinear) and discrete control.

The crane dynamics and the DC-motor were built in SIMULINK using continuous blocks with differnet levels of granularity.

The control is based on a linear observer which operates discrete. With observer matrices $\mathrm{A}, \mathrm{B}$ and $\mathrm{K}$ and observer state vector $\vec{q}$ control $\mathrm{v}$ is computed in the main by

$$
\begin{gathered}
\vec{q}_{n+1}=A \vec{q}_{n}+B\left[\begin{array}{ll}
v_{n} & x_{c, n}
\end{array}\right]^{T}, \quad y_{n}=K \vec{q}_{n} \\
v_{n+1}=k_{p}\left(\text { pos }_{\text {desired }}\left(x_{c, n}+r \alpha\right)\right)-y_{n+1}
\end{gathered}
$$

The controller algorithm and the diagnose functions were programmed in MATLAB and implemented in SIMULINK as a MATLAB function block. These blocks are supposed to work discrete. To assure that Zero-Order-Hold blocks are used. The angle sensor is simulated by a quantizer block and by comparing the current value to maximum and minimum defined in the control function (Figure 1).

The diagnosis is also implemented in form of a MATLAB-function. Fortunately Simulink allows different sample times for discrete blocks, so for the controller function a sample time of $10 \mathrm{~ms}$ and for the diagnosis functions a sample time of $1 \mathrm{~ms}$ is set.

\section{Task a: Comparison of Nonlinear and Linear model without Control}

The linear and nonlinear models were compared without brake and controller, so that from the model in Figure 1 only the nonlinear submodel and the linear submodel for the crane crab were used, without feedback control, only with given input $f_{c}$ and disturbances $f d$.

The nonlinear model is an implicit. Before implementation, the model was transformed symbolically into an explicit state space model (with four states for the crane crab, and one state for the motor). As the nonlinearities are relatively comprehensive (after making the model explicit), no basic Simulink blocks were used: the nonlinearities were calculated in a MATLAB function, and fed into a Simulink integrator block.

The dynamic calculations are done by an ODE45solver which is the standard MATLAB solver for nonstiff ODEs. Below the steady-state difference of $x_{l}$ and $f_{d}$ disturbance is shown.

\begin{tabular}{cccc}
\hline $\boldsymbol{f}_{\boldsymbol{d}}$ disturbance & -750 & -800 & -850 \\
\hline $\boldsymbol{x}_{\boldsymbol{l}}$ difference & -5.3086 & -6.8225 & -7.8131 \\
\hline
\end{tabular}

Table 1. A-Task: steady state difference

\section{Task b: Simulation of the Linear Fully Controlled System}

The task was to simulate the fully controlled system in a given scenario. The car starts out at position zero with no external force affecting it and with the desired position +3 . After 16 seconds the target position is changed to -0.5 and then after 36 seconds changed again to 3.8 . At time $t=42 f_{d}$ is set to -200 for 1 second and then to zero. The simulation stops at 60 seconds.

The controller and the brake are implemented via the DC-motor interacting with the linear model (Figure 1) following a linear discrete observer control.

The signal $d_{c}$ of the model stands for the friction coefficient of the car. To implement the brake, the value of $\mathrm{d}_{\mathrm{c}}$ is increased to simulate a mechanical brake. Again, the brake mechanism is described in the MATLAB controller function. When the break is applied the internal control parameters are held constant and the VC output is set to zero. In the linear model the instant stop of the car is achieved by increasing the friction coefficient to $10000 \mathrm{~s}^{-1}$. 
The dynamic calculations are done by an ODE45solver which is the standard MATLAB solver for nonstiff ODEs, and which also works well with discrete model parts. The simulation results are shown in Figure 2 and Figure 3.

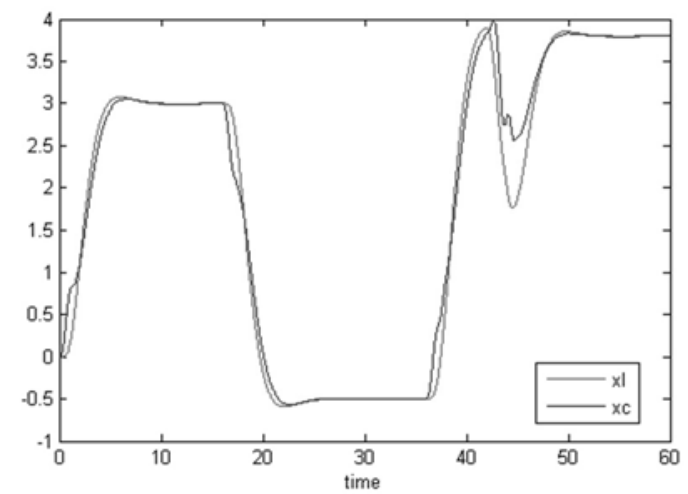

Figure 2. B-Task: Output parameters $\mathrm{xl}$ and $\mathrm{xc}$ over time, reacting to changing input signal PosDesired.

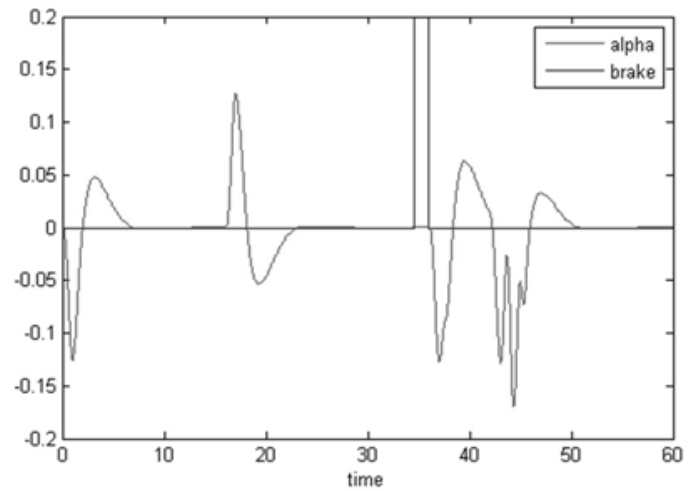

Figure 3. B-Task: Brake is activated at time $=34.59 \mathrm{~s}$ and resetted at time $=36 \mathrm{~s}$.

\section{Task c: Simulation of Controlled System with Sensor Diagnosis}

In this task the sensor diagnosis was added to the system. During the 60 second simulation time the desired position is shifted from +3 to -0.5 . After 18 seconds the angle sensor is forced to trigger the Emergencymode. Then at $\mathrm{t}=36$ the desired position is changed again to 3.8 after which $\mathrm{f}_{\mathrm{d}}$ is set to 200 for $1 \mathrm{~s}$, before resetting it to zero.

The diagnosis is described in a MATLAB block with a sample time of $1 \mathrm{~ms}$. The range sensors are programmed in the diagnosis MATLAB function.
If the range sensors for the maximum or minimum position are activated for more than $20 \mathrm{~ms}$ during a 100 ms time period the Emergencystop is initiated. To assure that, every millisecond the states of the range sensors are stored in an array. It has exactly 100 slots and if the sum exceeds 20 the Emergencystop is set.

The Emergencymode is handled the same way, except that it is activated when the position limits are exceeded for more than $50 \mathrm{~ms}$.

The break is applied when the car reaches its maximum position and the Emergencystop is activated. The load oscillates after the stop (results see Figure 4 and Figure 5.

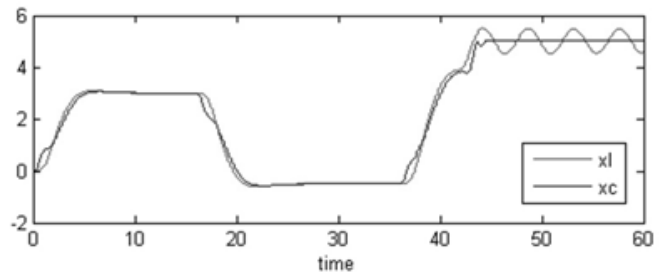

Figure 4. C-Task: Output parameters $\mathrm{xl}$ and $\mathrm{xc}$ over time, reacting to changing input signal PosDesired.

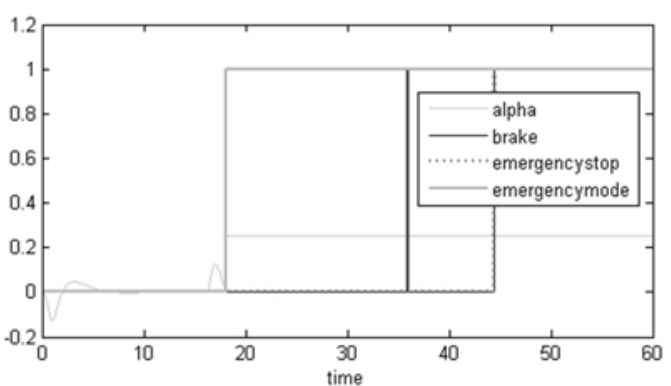

Figure 5. C-Task: Reaction of the model to broken angle sensor.

The interaction between the controller and the model is extremely sensible. Minor changes of the circle time have a great impact. They result in a decreased performance of the control.

\section{References}

[1] http://www.mathworks.de/de/help/matlab/learn matlab/ product-description.html

[2] http://www.mathworks.de/products/simulink/

[3] Scheikl J, Breitenecker F, Bausch-Gall I. C13 Crane and Embedded Control Revised Defintion. SNE 35/36, p 6971, December 2002 


\section{ASIM - Buchreihen / ASIM Book Series}

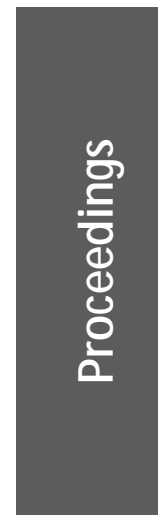

Simulation in Production und Logistics 2015 - 16. ASIM -Fachtagung Simulation in Produktion und Logistik M. Raabe, U. Clausen (Hrsg.); ISBN 978-3-8396-0936-1, Stuttgart: Fraunhofer Verlag, 2015. (O. P.)

Simulation in Produktion und Logistik 2013: Entscheidungsunterstützung von der Planung bis zur Steuerung W. Dangelmaier, C. Laroque, A. Klaas (Hrsg.); ISBN 978-3-942647-35-9, HNI-Verlagsschriftenreihe, Heinz Nixdorf Institut, Paderborn, 2013 (O. P.)

Modellierung, Regelung und Simulation in Automotive und Prozessautomation - Proc. 5. ASIM-Workshop Wismar 2011. C. Deatcu, P. Dünow, T. Pawletta, S. Pawletta (eds.), ISBN 978-3-901608-36-0, ASIM / ARGESIM, Wien, 2011 (O. A.)

Simulation in Produktion und Logistik 2010: Integrationsaspekte der Simulation - Technik, Organisation und Personal. G. Zülch, P. Stock, (Hrsg.), ISBN 978-3-86644-558-1, KIT Scientific Publ. Karlsruhe, 2010 (O.P.)

Simulation und Optimierung in Produktion und Logistik - Praxisorientierter Leitfaden mit Fallbeispielen. L. März, W. Krug, O. Rose, G. Weigert, G. (Hrsg.); ISBN 978-3-642-14535-3, Springer, 2011 (O.P.)

Verifikation und Validierung für die Simulation in Produktion und Logistik - Vorgehensmodelle und Techniken. M. Rabe, S. Spieckermann, S. Wenzel (eds.); ISBN: 978-3-540-35281-5, Springer, Berlin, 2008 (O. P.)

Qualitätskriterien für die Simulation in Produktion und Logistik - Planung und Durchführung von Simulationsstudien. S. Wenzel, M. Weiß, S. Collisi - Böhmer, H. Pitsch, O. Rose (Hrsg.); ISBN: 978-3-540-35281-5, Springer, Berlin, 2008

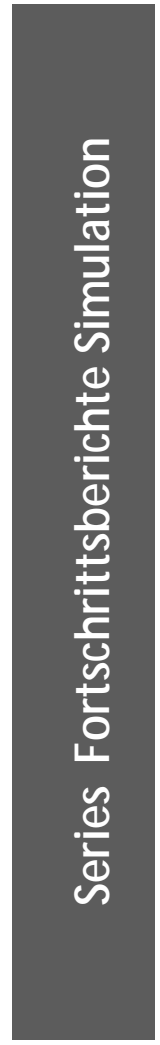

P. Einzinger: A Comparative Analysis of System Dynamics and Agent-Based Modelling for Health Care Reimbursement Systems. FBS 25, ASIM/ARGESIM Vienna, 2014; ISBN 978-3-901608-75-9, ARGESIM Report 75 (0. A.)

M. Bruckner: Agentenbasierte Simulation von Personenströmen mit unterschiedlichen Charakteristiken FBS 24, ASIM / ARGESIM Vienna, 2014; ISBN 978-3-901608-74-2, ARGESIM Report 74 (O. A.)

S. Emrich: Deployment of Mathematical Simulation Models for Space Management FBS 23, ASIM/ARGESIM Vienna, 2013; ISBN 978-3-901608-73-5, ARGESIM Report 73 (0. A.)

G. M aletzki: Rapid Control Prototyping komplexer und flexibler Robotersteuerungen auf Basis des SBC-Ansatzes. FBS 22, ASIM/ARGESIM Vienna, 2014; ISBN 978-3-901608-72-8, ARGESIM Report 72 (0. A.)

$X$. Descovich: Lattice Boltzmann Modeling and Simulation of Incompressible Flows in Distensible Tubes for Applications in Hemodynamics FBS 21, ASIM / ARGESIM Vienna, 2012; ISBN 978-3-901608-71-1, ARGESIM Report 71 (0. A.)

F. M iksch: Mathematical Modeling for New Insights into Epidemics by Herd Immunity and Serotype Shift FBS 20, ASIM/ARGESIM Vienna, 2012; ISBN 978-3-901608-70-4, ARGESIM Report 70 (0. A.)

S. Tauböck: Integration of Agent Based Modelling in DEVS for Utilisation Analysis: The MoreSpace Project at TU Vienna; FBS 19, ASIM/ARGESIM Vienna, 2012; ISBN 978-3-901608-69-8, ARGESIM Report 69 (O. A.)

Ch. Steinbrecher: Ein Beitrag zur prädiktiven Regelung verbrennungsmotorischer Prozesse FBS 18, ASIM / ARGESIM Vienna, 2010; ISBN 978-3-901608-68-1, ARGESIM Report 68 (O. A.)

O. Hagendorf: Simulation-based Parameter and Structure Optimisation of Discrete Event Systems FBS 17, ASIM/ARGESIM Vienna, 2010; ISBN 978-3-901608-67-4, ARGESIM Report 67 (0. A.)

Orders via ASIM (O. A.) or via Publisher (O. P.):

ASIM / ARGESIM Office Germany, Hochschule Wismar, PF 1210, 23952 Wismar, Germany

ASIM/ARGESIM Geschäftsstelle Österreich, c/ o DWH, Neustiftgasse 57, 1040 Vienna, Austria

Download (some books) via ASIM webpage in preparation

Info: www.asim-gi.org, info@asim-gi.org 


\section{SNE Simulation News}

\section{EUROSIM Data and Quick Info}
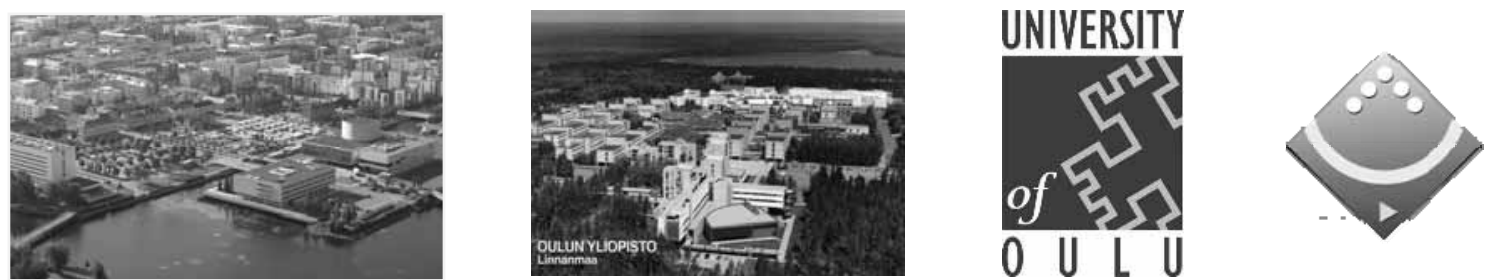

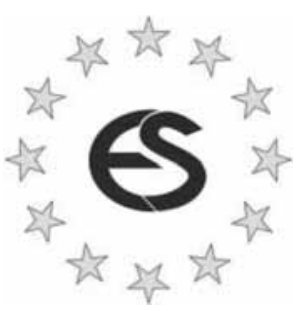

Contents

Info EUROSIM 2

Info EUROSIM Societies $3-8$

Info ASIM, CAE-SM SG 3

Info CROSSIM . CSSS, DBSS, FRANCOSIM

Info HSS, ISCS, LIOPHANT.

4

Info LSS, PSCS, SIMS, SLOSIM .

Info UKSIM , KA-SIM , ROM SIM

Info RNSS, Info SNE

Simulation Notes Europe SNE is the official membership journal of EUROSIM and distributed / available to members of the EUROSIM Societies as part of the membership benefits. SNE is published in a printed version (Print ISSN 2305-9974) and in an online version (Online ISSN 2306-0271). With Online SNE the publisher ARGESIM follows the Open Access strategy for basic SNE contributions. Since 2012 Online SNE contributions are identified by DOI 10.11128 /sne.xx.nnnnn. for better web availability and indexing.

Print SNE, high-resolution Online SNE, and additional SNE contributions are available via membership in a EUROSIM society.

This EUROSIM Data \& Quick Info compiles data from EUROSIM societies and groups: addresses, weblinks, officers of societies with function and email, to be published regularly in SNE issues.

\section{SNE Reports Editorial Board}

EUROSIM Esko Juuso,esko.juuso@oulu.fi

Borut Zupančič, borut.zupancic@fe.uni-lj.si

Felix Breitenecker, Felix.Breitenecker@tuwien.ac.at

ASIM Thorsten Pawletta,pawel@mb.hs-wismar.de

CAE-SMSG Emilio Jiminez, emilio.jiminez@unirioja.es

CROSSIM Vesna Dušak,vdusak@foi.hr

CSSS Mikuláš Alexík, alexik@frtk.utc.sk

DBSS A. Heemink, a.w.heemink@its.tudelft.nl

FRANCOSIM Karim Djouani, djouani@u-pec.fr

HSS András Jávor, javor@eik.bme.hu

ISCS M. Savastano, mario.savastano@unina.it

LIOPHANT F. Longo,f.longo@unical.it

LSS Yuri Merkuryev, merkur@itl.rtu.lv

PSCS Zenon Sosnowski, zenon@ii.pb.bialystok.pl

SIMS Esko Juuso, esko.juuso@oulu.fi

SLOSIM Rihard Karba, rihard.karba@fe.uni-lj.si

UKSIM Richard Zobel, r.zobel@ntlworld.com

KA-SIM Edmnd Hajrizi, info@ka-sim.com

ROMSIM Florin Stanciulescu, sflorin@ici.ro

RNSS Y. Senichenkov, sneyb@dcn.infos.ru

\section{SNE Editorial Office / ARGESIM}

$\rightarrow$ www.sne-journal.org, www.eurosim.info

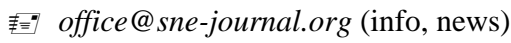

莑" eic@sne-journal.orgt Felix Breitenecker (publications)

If you have any information, announcement, etc. you want to see published, please contact a member of the editorial board in your country or the editorial office. For scientific publications, please contact the EiC. 


\section{EUROSIM}

\section{$\boldsymbol{S}$ * Federation of European Simulation Societies}

General Information. EUROSIM, the Federation of European Simulation Societies, was set up in 1989. The purpose of EUROSIM is to provide a European forum for simulation societies and groups to promote advancement of modelling and simulation in industry, research, and development. $\rightarrow$ www.eurosim.info

Member Societies. EUROSIM members may be national simulation societies and regional or international societies and groups dealing with modelling and simulation. At present EUROSIM has fourteen Full Members and three Observer Members:

\begin{tabular}{|c|c|}
\hline ASIM & $\begin{array}{l}\text { Arbeitsgemeinschaft Simulation } \\
\text { Austria, Germany, Switzerland }\end{array}$ \\
\hline CEA-SMSG & $\begin{array}{l}\text { Spanish M odelling and Simulation Group } \\
\text { Spain }\end{array}$ \\
\hline CROSSIM & $\begin{array}{l}\text { Croatian Society for Simulation M odeling } \\
\text { Croatia }\end{array}$ \\
\hline CSSS & $\begin{array}{l}\text { Czech and Slovak Simulation Society } \\
\text { Czech Republic, Slovak Republic }\end{array}$ \\
\hline DBSS & $\begin{array}{l}\text { Dutch Benelux Simulation Society } \\
\text { Belgium, Netherlands }\end{array}$ \\
\hline FRANCOSIM & $\begin{array}{l}\text { Société Francophone de Simulation } \\
\text { Belgium, France }\end{array}$ \\
\hline HSS & $\begin{array}{l}\text { Hungarian Simulation Society } \\
\text { Hungary }\end{array}$ \\
\hline ISCS & $\begin{array}{l}\text { Italian Society for Computer Simulation } \\
\text { Italy }\end{array}$ \\
\hline LIOPHANT & $\begin{array}{l}\text { LIOPHANT Simulation Club } \\
\text { Italy \& International, Observer M ember }\end{array}$ \\
\hline LSS & $\begin{array}{l}\text { Latvian Simulation Society } \\
\text { Latvia }\end{array}$ \\
\hline PSCS & $\begin{array}{l}\text { Polish Society for Computer Simulation } \\
\text { Poland }\end{array}$ \\
\hline SIMS & $\begin{array}{l}\text { Simulation Society of Scandinavia } \\
\text { Denmark, Finland, Norway, Sweden }\end{array}$ \\
\hline SLOSIM & $\begin{array}{l}\text { Slovenian Simulation Society } \\
\text { Slovenia }\end{array}$ \\
\hline UKSIM & $\begin{array}{l}\text { United Kingdom Simulation Society } \\
\text { UK, Ireland }\end{array}$ \\
\hline KA-SIM & $\begin{array}{l}\text { Romanian Society for M odelling and Sim- } \\
\text { ulation, Romania, Observer M ember }\end{array}$ \\
\hline ROMSIM & $\begin{array}{l}\text { Romanian Society for M odelling and Sim- } \\
\text { ulation, Romania, Observer M ember }\end{array}$ \\
\hline RNSS & $\begin{array}{l}\text { Russian National Simulation Society } \\
\text { Russian Federation, Observer M ember }\end{array}$ \\
\hline
\end{tabular}

EUROSIM Board / Officers. EUROSIM is governed by a board consisting of one representative of each member society, president and past president, and representatives for SNE Simulation notes Europe. The President is nominated by the society organising the next EUROSIM Congress. Secretary and Treasurer are elected out of members of the Board.

\begin{tabular}{ll}
\hline President & $\begin{array}{l}\text { Esko Juuso (SIM S) } \\
\text { esko.juuso@ oulu.fi }\end{array}$ \\
\hline Past President & $\begin{array}{l}\text { Khalid Al.Begain (UKSIM) } \\
\text { kbegain@ glam.ac.uk }\end{array}$ \\
\hline Secretary & $\begin{array}{l}\text { Borut Zupančič (SLOSIM) } \\
\text { borut.zupancic@fe.uni-lj.si }\end{array}$ \\
\hline Treasurer & $\begin{array}{l}\text { Felix Breitenecker (ASIM) } \\
\text { felix.breitenecker@tuwien.ac.at }\end{array}$ \\
\hline SNE Repres. & $\begin{array}{l}\text { Felix Breitenecker } \\
\text { felix.breitenecker@tuwien.ac.at }\end{array}$ \\
\hline
\end{tabular}

SNE - Simulation Notes Europe. SNE is a scientific journal with reviewed contributions as well as a membership newsletter for EUROSIM with information from the societies in the News Section. EUROSIM societies are offered to distribute to their members the journal SNE as official membership journal. SNE Publishers are EUROSIM, ARGESIM and ASIM.

\section{Editor-in-chief Felix Breitenecker \\ felix.breitenecker@tuwien.ac.at}

$\rightarrow$ www.sne-journal.org,

麦=; office@sne-journal.org

EUROSIM Congress. EUROSIM is running the triennial conference series EUROSIM Congress. The congress is organised by one of the EUROSIM societies.

EUROSIM 2016 will be organised by SIMS in Oulu, Finland, September 16-20, 2016.

\section{Chairs / Team EUROSIM 2016}

Esko Juuso EUROSIM President, esko.juuso@oulu.fi Erik Dahlquist SIMS President, erik.dahlquist@mdh.se Kauko Leiviskä EUROSIM 2016 Chair, kauko.leiviska@oulu.fi

$\rightarrow$ www.eurosim.info

奉”。ffice@automaatioseura.fi 


\section{EUROSIM Member Societies}

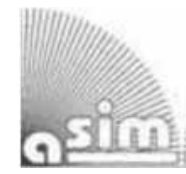

ASIM

\section{German Simulation Society \\ Arbeitsgemeinschaft Simulation}

ASIM (Arbeitsgemeinschaft Simulation) is the association for simulation in the German speaking area, servicing mainly Germany, Switzerland and Austria. ASIM was founded in 1981 and has now about 700 individual members, and 30 institutional or industrial members.

$\rightarrow$ www.asim-gi.org with members' area

豐info@asim-gi.org,admin@asim-gi.org

$\bowtie$ ASIM - Inst. f. Analysis and Scientific Computing

Vienna University of Technology

Wiedner Hauptstraße 8-10, 1040 Vienna, Austria

\begin{tabular}{|c|c|}
\hline \multicolumn{2}{|l|}{ ASIM Officers } \\
\hline President & $\begin{array}{l}\text { Felix Breitenecker } \\
\text { felix.breitenecker@tuwien.ac.at }\end{array}$ \\
\hline \multirow[t]{2}{*}{ Vice presidents } & Sigrid Wenzel, s.wenzel@uni-kassel.de \\
\hline & T. Pawletta, pawel@mb.hs-wismar.de \\
\hline Secretary & Ch. Deatcu, christina.deatcu@ hs-wismar.de \\
\hline Treasurer & Anna Mathe, anna.mathe@tuwien.ac.at \\
\hline \multirow{4}{*}{$\begin{array}{l}\text { Membership } \\
\text { Affairs }\end{array}$} & S. Wenzel, s.wenzel@ uni-kassel.de \\
\hline & W. M aurer, werner.maurer@zhwin.ch \\
\hline & Ch. Deatcu, christina.deatcu@ hs-wismar.de \\
\hline & F. Breitenecker, felix.breitenecker@tuwien.ac.at \\
\hline \multirow{4}{*}{$\begin{array}{l}\text { Universities / } \\
\text { Research Inst. }\end{array}$} & S. Wenzel, s.wenzel@ uni-kassel.de \\
\hline & W.Wiechert, W.Wiechert@fz-juelich.de \\
\hline & J. Haase, Joachim.Haase@ eas.iis.fraunhofer.de \\
\hline & Katharina Nöh, k.noeh@ fz-juelich.de \\
\hline \multirow[t]{2}{*}{ Industry } & S. Wenzel, s.wenzel@uni-kassel.de \\
\hline & K. Panreck, Klaus.Panreck@ hella.com \\
\hline \multirow[t]{2}{*}{ Conferences } & Klaus Panreck Klaus.Panreck@ hella.com \\
\hline & J.Wittmann, wittmann@ htw-berlin.de \\
\hline \multirow[t]{3}{*}{ Publications } & Th. Pawletta, pawel@mb.hs-wismar.de \\
\hline & Christina Deatcu, christina.deatcu@ hs-wismar.de \\
\hline & F. Breitenecker, felix.breitenecker@tuwien.ac.at \\
\hline \multirow[t]{2}{*}{ Repr. EUROSIm } & F. Breitenecker, felix.breitenecker@tuwien.ac.at \\
\hline & N. Popper, niki.popper@drahtwarenhandlung.at \\
\hline \multirow{3}{*}{$\begin{array}{l}\text { Education / } \\
\text { Teaching }\end{array}$} & A. Körner, andreas.koerner@tuwien.ac.at \\
\hline & N. Popper, niki.popper@drahtwarenhandlung.at \\
\hline & Katharina Nöh, k.noeh@fz-juelich.de \\
\hline \multirow{2}{*}{$\begin{array}{l}\text { International } \\
\text { Affairs }\end{array}$} & A. Körner, andreas.koerner@tuwien.ac.at \\
\hline & 0. Rose, Oliver.Rose@tu-dresden.de \\
\hline \multirow{2}{*}{$\begin{array}{l}\text { Editorial Board } \\
\text { SNE }\end{array}$} & T. Pawletta, pawel@mb.hs-wismar.de \\
\hline & Ch. Deatcu, christina.deatcu@ hs-wismar.de \\
\hline Web EUROSIM & Anna Mathe, anna.mathe@tuwien.ac.at \\
\hline
\end{tabular}

ASIM Working Committee. ASIM, part of GI - Gesellschaft für Informatik, is organised in Working Committees, dealing with applications and comprehensive subjects in modelling and simulation:

\begin{tabular}{cl} 
ASIM Working Committee \\
\hline GM MS & $\begin{array}{l}\text { Methods in Modelling and Simulation } \\
\text { Th. Pawletta, pawel@ mb.hs-wismar.de }\end{array}$ \\
\hline SUG & $\begin{array}{l}\text { Simulation in Environmental Systems } \\
\text { Wittmann, wittmann@ informatik.uni-hamburg.de }\end{array}$ \\
\hline STS & $\begin{array}{l}\text { Simulation of Technical Systems } \\
\text { H.T.M ammen, Heinz-Theo.M ammen@ hella.com }\end{array}$ \\
\hline SPL & $\begin{array}{l}\text { Simulation in Production and Logistics } \\
\text { Sigrid Wenzel, s.wenzel@ uni-kassel.de }\end{array}$ \\
\hline EDU & $\begin{array}{l}\text { Simulation in Education/Education in Simulation } \\
\text { N. Popper, niki.popper@dwh.at } \\
\text { A. Körner, andreas.koerner@tuwien.ac.at }\end{array}$ \\
\hline & $\begin{array}{l}\text { Working Groups for Simulation in Business Admin- } \\
\text { istration, in Traffic Systems, for Standardisation, for } \\
\text { Validation, etc. }\end{array}$ \\
\hline
\end{tabular}

\section{CEA-SM SG - Spanish Modelling and Simulation Group}

CEA is the Spanish Society on Automation and Control In order to improve the efficiency and to deep into the different fields of automation, the association is divided into thematic groups, one of them is named 'Modelling and Simulation', constituting the group.

$\rightarrow$ www.cea-ifac.es/wwwgrupos/simulacion

$\rightarrow$ simulacion@cea-ifac.es

$\triangle$ CEA-SMSG / María Jesús de la Fuente,

System Engineering and AutomaticControl department, University of Valladolid,

Real de Burgos s/n., 47011 Valladolid, SPAIN

\begin{tabular}{ll}
\hline \multicolumn{2}{l}{ CAE - SM SG Officers } \\
\hline President & M. A. Piera Eroles, M iquelAngel.Piera@uab.es \\
\hline Vice president & Emilio Jiminez, emilio.jiminez@unirioja.es \\
\hline Repr. EURoSIm & Emiliojiminez, emilio.jiminez@unirioja.es \\
\hline Edit. Board SNE & Emiliojiminez, emilio.jiminez@unirioja.es \\
\hline Web EURoSIM & Mercedes Peres, mercedes.perez@unirioja.es \\
\hline
\end{tabular}
Last data update December2013 


\section{CROSSIM - Croatian Society for Simulation Modelling}

CROSSIM-Croatian Society for Simulation Modelling was founded in 1992 as a non-profit society with the goal to promote knowledge and use of simulation methods and techniques and development of education. CROSSIM is a full member of EUROSIM since 1997.

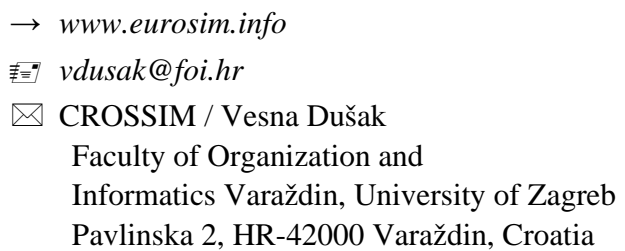

\begin{tabular}{ll}
\multicolumn{2}{l}{ CROSSIM Officers } \\
\hline President & Vesna Dušak, vdusak@foi.hr \\
\hline Vice president & Jadranka Božikov, jbozikov@snz.hr \\
\hline Secretary & Vesna Bosilj-Vukšić, vbosilj@ efzg.hr \\
\hline Executive board & Vlatko Čerić, vceric@ efzg.hr \\
\cline { 2 - 2 } members & Tarzan Legović, legovic@ irb.hr \\
\hline Repr. EuRoSım & Jadranka Božikov, jbozikov@snz.hr \\
\hline Edit. Board SNE & Vesna Dušak, vdusak@ foi.hr \\
\hline Web EuRoSıM & Jadranka Bozikov, jbozikov@ snz.hr \\
\hline
\end{tabular}

\section{\begin{tabular}{|l|l|}
\hline CSSS & $\begin{array}{l}\text { CSSS - Czech and Slovak } \\
\text { Simulation Society }\end{array}$ \\
\hline
\end{tabular}}

CSSS -The Czech and Slovak Simulation Society has about 150 members working in Czech and Slovak national scientific and technical societies (Czech Society for Applied Cybernetics and Informatics, Slovak Society for Applied Cybernetics and Informatics). The main objectives of the society are: development of education and training in the field of modelling and simulation, organising professional workshops and conferences, disseminating information about modelling and simulation activities in Europe. Since 1992, CSSS is full member of EUROSIM.

$\rightarrow$ www.fit.vutbr.cz/CSSS

㪯丮snorek@fel.cvut.cz

$\triangle$ CSSS / Miroslav Šnorek, CTU Prague

FEE, Dept. Computer Science and Engineering,

Karlovo nam. 13, 12135 Praha 2, Czech Republic

\begin{tabular}{ll}
\hline CSSS Officers & \\
\hline President & Miroslav Šnorek, snorek@fel.cvut.cz \\
\hline Vice president & Mikuláš Alexík, alexik@frtk.fri.utc.sk \\
\hline Treasurer & Evžen Kindler, ekindler@centrum.cz \\
\hline Scientific Secr. & A. Kavička, Antonin.Kavicka@upce.cz \\
\hline Repr. EuroSim & Miroslav Šnorek, snorek@fel.cvut.cz \\
\hline Deputy & Mikuláš Alexík, alexik@frtk.fri.utc.sk \\
\hline Edit. Board SNE & Mikuláš Alexík, alexik@frtk.fri.utc.sk \\
\hline Web EURoSIM & Petr Peringer, peringer@fit.vutbr.cz \\
\hline
\end{tabular}

\section{DBSS - Dutch Benelux Simulation Society}

The Dutch Benelux Simulation Society (DBSS) was founded in July 1986 in order to create an organisation of simulation professionals within the Dutch language area. DBSS has actively promoted creation of similar organisations in other language areas. DBSS is a member of EUROSIM and works in close cooperation with its members and with affiliated societies.

$\rightarrow$ ww. eurosim.info

䒠=”.w.heemink@its.tudelft.nl

$\triangle$ DBSS / A. W. Heemink

Delft University of Technology, ITS - twi,

Mekelweg 4, 2628 CD Delft, The Netherlands

\section{DBSS Officers}

\begin{tabular}{ll}
\hline President & A. Heemink, a.w.heemink@ its.tudelft.nl \\
\hline Vice president & W. Smit, smitnet@wxs.nl \\
\hline Treasurer & W. Smit, smitnet@wx.nl \\
\hline Secretary & W. Smit, smitnet@wxs.nl \\
\hline Repr. EuroSIm & A. Heemink, a.w.heemink@ its.tudelft.nl \\
\hline Deputy & W. Smit, smitnet@wxs.nl \\
\hline Edit. Board SNE & A. Heemink, a.w.heemink@ its.tudelft.nl \\
\hline
\end{tabular}

\section{FRANCOSIM - Société Francophone de Simulation}

FRANCOSIM was founded in 1991 and aims to the promotion of simulation and research, in industry and academic fields. Francosim operates two poles.

- Pole Modelling and simulation of discrete event systems. Pole Contact: Henri Pierreval, pierreva@imfa.fr

- Pole Modelling and simulation of continuous systems. Pole Contact: Yskandar Hamam, y.hamam@esiee.fr 
$\rightarrow$ www.eurosim.info

莑”y.hamam@esiee.fr

$\triangle$ FRANCOSIM / Yskandar Hamam

Groupe ESIEE, Cité Descartes,

BP 99, 2 Bd. Blaise Pascal,

93162 Noisy le Grand CEDEX, France

\section{FRANCOSIM Officers}

\begin{tabular}{ll}
\hline President & Karim Djouani, djouani@u-pec.fr \\
\hline Treasurer & François Rocaries, f.rocaries@esiee.fr \\
\hline Repr. EuRoSım & Karim Djouani, djouani@u-pec.fr \\
\hline Edit. Board SNE & Karim Djouani, djouani@u-pec.fr \\
\hline
\end{tabular}

Last data update December2012

\section{HSS - Hungarian Simulation Society}

The Hungarian Member Society of EUROSIM was established in 1981 as an association promoting the exchange of information within the community of people involved in research, development, application and education of simulation in Hungary and also contributing to the enhancement of exchanging information between the Hungarian simulation community and the simulation communities abroad. HSS deals with the organization of lectures, exhibitions, demonstrations, and conferences.

$\rightarrow$ www.eurosim.info

莑” javor@eik.bme.hu

$\triangle$ HSS / András Jávor,

Budapest Univ. of Technology and Economics,

Sztoczek u. 4, 1111 Budapest, Hungary

\begin{tabular}{ll} 
HSS Officers & \\
\hline President & András Jávor, javor@ eik.bme.hu \\
\hline Vice president & Gábor Szúcs, szucs@itm.bme.hu \\
\hline Secretary & Ágnes Vigh, vigh@itm.bme.hu \\
\hline Repr. EuRoSIm & András Jávor, javor@ eik.bme.hu \\
\hline Deputy & Gábor Szúcs, szucs@itm.bme.hu \\
\hline Edit. Board SNE & András Jávor, javor@ eik.bme.hu \\
\hline Web EuRoSım & Gábor Szúcs, szucs@itm.bme.hu \\
\hline
\end{tabular}

\section{ISCS - Italian Society for Computer Simulation}

The Italian Society for Computer Simulation (ISCS) is a scientific non-profit association of members from industry, university, education and several public and research institutions with common interest in all fields of computer simulation. $\rightarrow$ www.eurosim.info

莑丮 Mario.savastano@uniina.at

$\triangle$ ISCS / Mario Savastano, c/o CNR - IRSIP,

Via Claudio 21, 80125 Napoli, Italy

\begin{tabular}{ll}
\hline ISCS Officers & \\
\hline President & M. Savastano, mario.savastano@ unina.it \\
\hline Vice president & F. Maceri, Franco.M aceri@uniroma2.it \\
\hline Repr. EUROSIM & F. Maceri, Franco.M aceri@uniroma2.it \\
\hline Secretary & $\begin{array}{l}\text { Paola Provenzano, } \\
\text { paola.provenzano@ uniroma2.it }\end{array}$ \\
\hline Edit. Board SNE & M. Savastano, mario.savastano@ unina.it \\
\hline
\end{tabular}

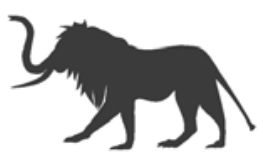

LIOPHANT Simulation

Liophant Simulation is a non-profit association born in order to be a trait-d'union among simulation developers and users; Liophant is devoted to promote and diffuse the simulation techniques and methodologies; the Association promotes exchange of students, sabbatical years, organization of International Conferences, organization of courses and stages in companies to apply the simulation to real problems.

$\rightarrow$ www.liophant.org

莑”info@liophant.org

$\triangle$ LIOPHANT Simulation, c/o Agostino G. Bruzzone, DIME, University of Genoa, Polo Savonese, via Molinero 1, 17100 Savona (SV), Italy

\begin{tabular}{ll}
\hline LOPHANT Officers \\
\hline President & A.G. Bruzzone, agostino@itim.unige.it \\
\hline Director & E. Bocca, enrico.bocca@ liophant.org \\
\hline Secretary & A. Devoti, devoti.a@ iveco.com \\
\hline Treasurer & Marina M asseimassei@ itim.unige.it \\
\hline Repr. EURoSIM & A.G. Bruzzone, agostino@itim.unige.it \\
\hline Deputy & F. Longo, f.longo@ unical.it \\
\hline Edit. Board SNE & F. Longo, f.longo@ unical.it \\
\hline Web EUROSIM & F. Longo, f.longo@ unical.it \\
\hline
\end{tabular}




\section{LSS - Latvian Simulation Society}

The Latvian Simulation Society (LSS) has been founded in 1990 as the first professional simulation organisation in the field of Modelling and simulation in the postSoviet area. Its members represent the main simulation centres in Latvia, including both academic and industrial sectors.

$\rightarrow$ briedis.itl.rtu.lv/imb/

䒠三”merkur@itl.rtu.lv

$\bowtie$ LSS / Yuri Merkuryev, Dept. of Modelling and Simulation Riga Technical University

Kalku street 1, Riga, LV-1658, LATVIA

\begin{tabular}{ll}
\hline LSS Officers & \\
\hline President & Yuri M erkuryev, merkur@itl.rtu.Iv \\
\hline Secretary & Artis Teilans, Artis.Teilans@ exigenservices.com \\
\hline Repr. EuROSIM & Yuri M erkuryev, merkur@itl.rtu.Iv \\
\hline Deputy & Artis Teilans, Artis.Teilans@exigenservices.com \\
\hline Edit. Board SNE & Yuri M erkuryev, merkur@itl.rtu.Iv \\
\hline Web EuRoSIM & Oksana Sosho, oksana@itl.rtu.Iv \\
\hline
\end{tabular}

Last data update December2013

\section{PSCS - Polish Society for Computer Simulation}

PSCS was founded in 1993 in Warsaw. PSCS is a scientific, non-profit association of members from universities, research institutes and industry in Poland with common interests in variety of methods of computer simulations and its applications. At present PSCS counts 257 members.

$\rightarrow$ www.ptsk.man.bialystok.pl

莑- leon@ibib.waw.pl

$\triangle$ PSCS / Leon Bobrowski, c/o IBIB PAN,

ul. Trojdena 4 (p.416), 02-109 Warszawa, Poland

\begin{tabular}{ll}
\hline PSCS Officers & \\
\hline President & Leon Bobrowski, leon@ ibib.waw.pl \\
\hline Vice president & Tadeusz Nowicki, \\
& Tadeusz.Nowicki@wat.edu.pl \\
\hline Treasurer & Z. Sosnowski, zenon@ii.pb.bialystok.pl \\
\hline Secretary & Zdzislaw Galkowski, \\
& Zdzislaw.Galkowski@ simr.pw.edu.pl \\
\hline Repr. EuroSım & Leon Bobrowski, leon@ ibib.waw.pl \\
\hline Deputy & Tadeusz Nowicki, tadeusz.nowicki@ wat.edu.pl \\
\hline Edit. Board SNE & Zenon Sosnowski, z.sosnowski@ pb.ed.pl \\
\hline Web EuroSım & Magdalena Topczewska \\
& m.topczewska@ pb.edu.pl \\
\hline
\end{tabular}

Last data update December2013

\section{SIM S - Scandinavian Simulation Society}

SIMS is the Scandinavian Simulation Society with members from the four Nordic countries Denmark, Finland, Norway and Sweden. The SIMS history goes back to 1959. SIMS practical matters are taken care of by the SIMS board consisting of two representatives from each Nordic country (Iceland one board member).

SIMS Structure. SIMS is organised as federation of regional societDjouaniies. There are FinSim (Finnish Simulation Forum), DKSIM (Dansk Simuleringsforening) and NFA (Norsk Forening for Automatisering).

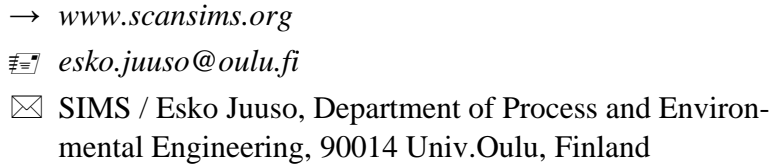

\begin{tabular}{ll}
\hline SIMS Officers & \\
\hline President & Esko Juuso, esko.juuso@ oulu.fi \\
\hline Vice president & Erik Dahlquist, erik.dahlquist@ mdh.se \\
\hline Treasurer & $\begin{array}{l}\text { Vadim Engelson, } \\
\text { vadim.engelson@ mathcore.com }\end{array}$ \\
\hline Repr. EUROSIM & Esko Juuso, esko.juuso@ oulu.fi \\
\hline Edit. Board SNE & Esko Juuso, esko.juuso@ oulu.fi \\
\hline Web EuRoSIM & Vadim Engelson, \\
& vadim.engelson@ mathcore.com \\
\hline
\end{tabular}
Last data update December2013

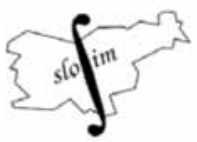

\section{SLOSIM - Slovenian Society for Simulation and Modelling}

SLOSIM - Slovenian Society for Simulation and Modelling was established in 1994 and became the full member of EUROSIM in 1996. Currently it has 69 members from both slovenian universities, institutes, and industry. It promotes modelling and simulation approaches to problem solving in industrial as well as in academic environments by establishing communication and cooperation among corresponding teams.

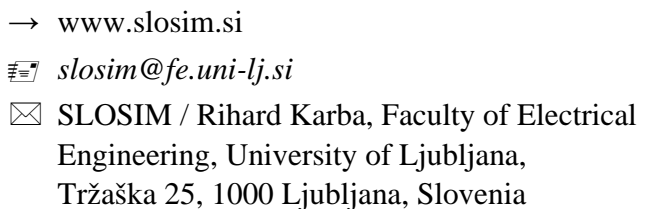




\begin{tabular}{|c|c|}
\hline SLOSIM Officers & \\
\hline President & Vito Logar, vito.logar@fe.uni-lj.si \\
\hline Vice president & Božidar Šarler, bozidar.sarler@ung.si \\
\hline Secretary & Aleš Belič, ales.belic@sandoz.com \\
\hline Treasurer & Milan Simčič, milan.simcic@fe.uni-lj.si \\
\hline Repr. EUROSIM & B. Zupančič, borut.zupancic@fe.uni-lj.si \\
\hline Deputy & Vito Logar, vito.logar@ fe.uni-lj.si \\
\hline Edit. Board SNE & Rihard Karba, rihard.karba@fe.uni-lj.si \\
\hline Web EuroSIM & Vito Logar, vito.logar@fe.uni-lj.si \\
\hline
\end{tabular}

\section{UKSIM - United Kingdom Simulation Society}

UKSIM has more than 100 members throughout the UK from universities and industry. It is active in all areas of simulation and it holds a biennial conference as well as regular meetings and workshops.

$\rightarrow$ www.uksim.org.uk

恝 david.al-dabass@ntu.ac.uk

$\bowtie$ UKSIM / Prof. David Al-Dabass

Computing \& Informatics,

Nottingham Trent University

Clifton lane, Nottingham, NG11 8NS

United Kingdom

\begin{tabular}{ll}
\hline UKSIM Officers & \\
\hline President & $\begin{array}{l}\text { David Al-Dabass, } \\
\text { david.al-dabass@ ntu.ac.uk }\end{array}$ \\
\hline Vice president & A. Orsoni, A.Orsoni@ kingston.ac.uk \\
\hline Secretary & Richard Cant, richard.cant@ ntu.ac.uk \\
\hline Treasurer & A. Orsoni, A.Orsoni@ kingston.ac.uk \\
\hline Membership chair & K. Al-Begain, kbegain@ glam.ac.uk \\
\hline Univ. liaison chair & R. Cheng, rchc@ maths.soton.ac.uk \\
\hline Repr. EuRoSIM & Richard Zobel, r.zobel@ ntlworld.com \\
\hline Deputy & K. Al-Begain, kbegain@ glam.ac.uk \\
\hline Edit. Board SNE & Richard Zobel, r.zobel@ ntlworld.com \\
\hline & \multicolumn{1}{c}{ Last data update December2013 }
\end{tabular}

\section{EUROSIM OBSERVER M EM BERS}

\section{KA-SIM Kosovo Simulation Society}

Kosova Association for Modeling and Simulation (KA SIM, founded in 2009), is part of Kosova Association of Control, Automation and Systems Engineering (KA CASE). KA - CASE was registered in 2006 as non Profit Organization and since 2009 is National Member of IFAC - International Federation of Automatic Control. KA-SIM joined EUROSIM as Observer Member in 2011.

KA-SIM has about 50 members, and is organizing the international conference series International Conference in Business, Technology and Innovation, in November, in Durrhes, Albania, an IFAC Simulation workshops in Pristina.

\section{$\rightarrow$ www.ubt-uni.net/ka-case

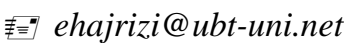 \\ $\triangle$ MOD\&SIM KA-CASE}

Att. Dr. Edmond Hajrizi

Univ. for Business and Technology (UBT)

Lagjja Kalabria p.n., 10000 Prishtina, Kosovo

\begin{tabular}{ll}
\multicolumn{2}{l}{ KA-SIM Officers } \\
\hline President & Edmond Hajrizi, ehajrizi@ubt-uni.net \\
\hline Vice president & M uzafer Shala, info@ka-sim.com \\
\hline Secretary & Lulzim Beqiri, info@ ka-sim.com \\
\hline Treasurer & Selman Berisha, info@ ka-sim.com \\
\hline Repr. EuRoSıM & Edmond Hajrizi, ehajrizi@ ubt-uni.net \\
\hline Deputy & M uzafer Shala, info@ ka-sim.com \\
\hline Edit. Board SNE & Edmond Hajrizi, ehajrizi@ ubt-uni.net \\
\hline Web EuROSIM & Betim Gashi, info@ ka-sim.com \\
\hline
\end{tabular}

\section{ROM SIM - Romanian Modelling and Simulation Society}

ROMSIM has been founded in 1990 as a non-profit society, devoted to theoretical and applied aspects of modelling and simulation of systems. ROMSIM currently has about 100 members from Romania and Moldavia.

$\rightarrow$ www.ici.ro/romsim/

麦=”sflorin@ici.ro

$\square$ ROMSIM / Florin Stanciulescu,

National Institute for Research in Informatics, Averescu Av. 8 - 10, 71316 Bucharest, Romania 


\begin{tabular}{ll}
\hline ROM SIM Officers \\
\hline President & Florin Stanciulescu, sflorin@ ici.ro \\
\hline Vice president & Florin Hartescu, flory @ici.ro \\
& Marius Radulescu, mradulescu@ ici.ro \\
\hline Repr. EUROSIM & Florin Stanciulescu, sflorin@ ici.ro \\
\hline Deputy & Marius Radulescu, mradulescu@ ici.ro \\
\hline Edit. Board SNE & Florin Stanciulescu, sflorin@ ici.ro \\
\hline Web EUROSIM & Zoe Radulescu, radulescu@ ici.ro \\
\hline & \multicolumn{1}{c}{ Last data update December2012 }
\end{tabular}

\section{RNSS - Russian Simulation Society}

NSS - The Russian National Simulation Society (Национальное Общество Имитационного Моделирования - НОИМ) was officially registered in Russian Federation on February 11, 2011. In February 2012 NSS has been accepted as an observer member of EUROSIM.

$\rightarrow$ www.simulation.su

莑”7yusupov@iias.spb.su

$\bowtie$ RNSS / R. M. Yusupov,

St. Petersburg Institute of Informatics and Automation

RAS, 199178, St. Petersburg, 14th lin. V.O, 39

\begin{tabular}{ll}
\hline RNSS Officers & \\
\hline President & R. M. Yusupov, yusupov@iias.spb.su \\
\hline Chair Man. Board & A. Plotnikov, plotnikov@sstc.spb.ru \\
\hline Secretary & M. Dolmatov, dolmatov@simulation.su \\
\hline Repr. EuRoSIM & R. M. Yusupov, yusupov@iias.spb.su \\
\hline Deputy & B. Sokolov, sokol@iias.spb.su \\
\hline Edit. Board SNE & Y. Senichenkov, sneyb@dcn.infos.ru \\
\hline &
\end{tabular}

\section{SNE - Simulation Notes Europe}

Simulation Notes Europe publishes peer reviewed Technical Notes, Short Notes and Overview Notes on developments and trends in modelling and simulation in various areas and in application and theory. Furthermore SNE documents the ARGESIM Benchmarks on Modelling Approaches and Simulation Implementations with publication of definitions, solutions and discussions (Benchmark Notes). Special Educational Notes present the use of modelling and simulation in and for education and for e-learning.
SNE is the official membership journal of EUROSIM, the Federation of European Simulation Societies. A News Section in SNE provides information for EUROSIM Simulation Societies and Simulation Groups. In 2013, SNE introduced an extended submission strategy i) individual submissions of scientific papers, and ii) submissions of selected contributions from conferences of EUROSIM societies for post-conference publication (suggested by conference organizer and authors) - both with peer review.

SNE is published in a printed version (Print ISSN 2305-9974) and in an online version (Online ISSN 2306-0271). With Online SNE the publisher ARGESIM follows the Open Access strategy, allowing download of published contributions for free. Since 2012 Online SNE contributions are identified by an DOI (Digital Object Identifier) assigned to the publisher ARGESIM (DOI prefix 10.11128). Print SNE, high-resolution Online SNE, source codes of the Benchmarks and other additional sources are available for subscription via membership in a EUROSIM society.

Authors Information. Authors are invited to submit contributions which have not been published and have not being considered for publication elsewhere to the SNE Editorial Office. SNE distinguishes different types of contributions (Notes):

- Overview Note - State-of-the-Art report in a specific area, up to 14 pages, only upon invitation

- Technical Note - scientific publication on specific topic in modelling and simulation, $6-8$ (10) pages

- Education Note - modelling and simulation in / for education and e-learning; max. 6 pages

- Short Note - recent development on specific topic, max. 4 pages

- Software Note - specific implementation with scientific analysis, max 4 pages

- Benchmark Note - Solution to an ARGESIM Benchmark;basic solution 2 pages, extended and commented solution 4 pages, comparative solutions on invitation

Interested authors may find further information at SNE's website $\rightarrow$ www.sne-journal.org (layout templates for Notes, requirements for benchmark solutions, etc.).

\section{SNE Editorial Office / ARGESIM \\ $\rightarrow$ www.sne-journal.org, www.eurosim.info \\ 暳: office@sne-journal.org (info, news) \\ 麦=icic@sne-journal.org Felix Breitenecker \\ (publications)}




\section{EUROSIM 2016 $9^{\text {th }}$ EUROSIM Congress on Modelling and Simulation}

City of Oulu, Finland, September 12 - 16, 2016
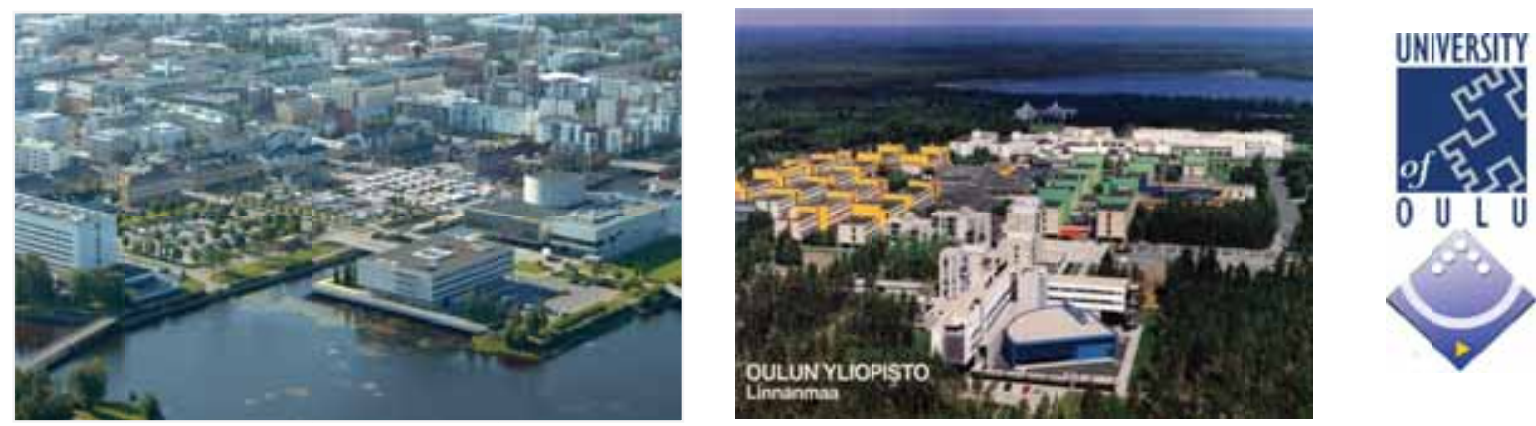

EUROSIM Congresses are the most important modelling and simulation events in Europe. For EUROSIM 2016, we are soliciting original submissions describing novel research and developments in the following (and related) areas of interest: Continuous, discrete (event) and hybrid modelling, simulation, identification and optimization approaches. Two basic contribution motivations are expected: M\&S Methods and Technologies and M\&S Applications. Contributions from both technical and non-technical areas are welcome.

Congress Topics The EUROSIM 2016 Congress will include invited talks, parallel, special and poster sessions, exhibition and versatile technical and social tours. The Congress topics of interest include, but are not limited to:

Intelligent Systems and Applications Hybrid and Soft Computing Data \& Semantic Mining Neural Networks, Fuzzy Systems \& Evolutionary Computation Image, Speech \& Signal Processing Systems Intelligence and Intelligence Systems Autonomous Systems Energy and Power Systems Mining and Metal Industry Forest Industry

Buildings and Construction Communication Systems

Circuits, Sensors and Devices

Security Modelling and Simulation
Bioinformatics, Medicine, Pharmacy and Bioengineering

Water and Wastewater Treatment, Sludge Management and Biogas Production

Condition monitoring, Mechatronics and maintenance

Automotive applications

e-Science and e-Systems

Industry, Business, Management, Human Factors and Social Issues

Virtual Reality, Visualization,

Computer Art and Games

Internet Modelling, Semantic Web and Ontologies

Computational Finance \& Economics
Simulation Methodologies and Tools Parallel and Distributed Architectures and Systems Operations Research Discrete Event Systems Manufacturing and Workflows Adaptive Dynamic Programming and Reinforcement Learning Mobile/Ad hoc wireless networks, mobicast, sensor placement, target tracking Control of Intelligent Systems Robotics, Cybernetics, Control Engineering, \& Manufacturing

Transport, Logistics, Harbour, Shipping and Marine Simulation

Congress Venue / Social Events The Congress will be held in the City of Oulu, Capital of Northern Scandinavia. The main venue and the exhibition site is the Oulu City Theatre in the city centre. Pre and Post Congress Tours include Arctic Circle, Santa Claus visits and hiking on the unique routes in Oulanka National Park.

Congress Team: The Congress is organised by SIMS - Scandinavian Simulation Society, FinSim - Finnish Simulation Forum, Finnish Society of Automation, and University of Oulu. Esko Juuso EUROSIM President, Erik Dahlquist SIMS President, Kauko Leiviskä EUROSIM 2016 Chair

Info: eurosim2016.automaatioseura.fi, office@automaatioseura.fi 


\section{Parlez-vous}

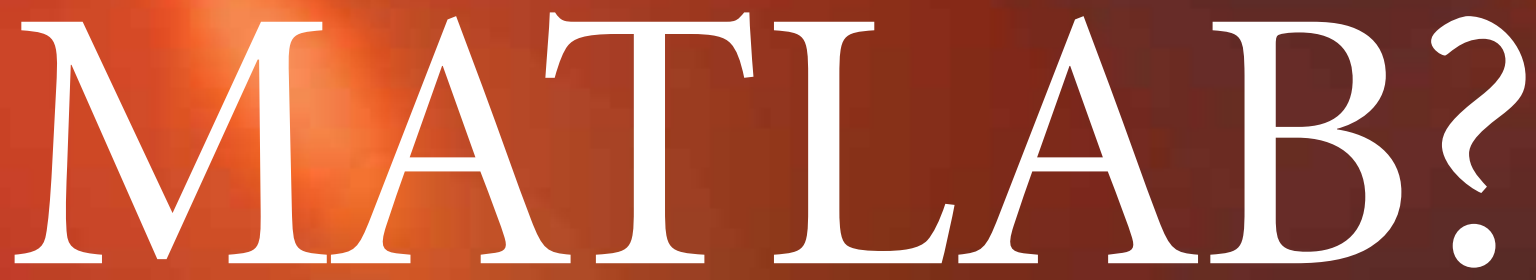

Über eine Million Menschen weltweit sprechen

MATLAB. Ingenieure und Wissenschaftler in

allen Bereichen - von der Luft- und Raumfahrt

über die Halbleiterindustrie bis zur Bio-

technologie, Finanzdienstleistungen und

Geo- und Meereswissenschaften - nutzen

MATLAB, um ihre Ideen auszudrücken.

Sprechen Sie MATLAB?

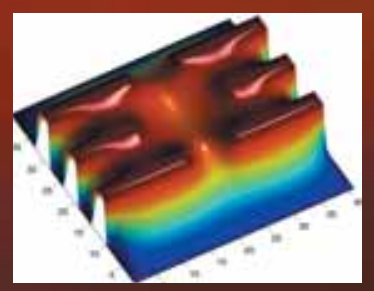

Modellierung eines elektrischen

Potentials in einem Quantum Dot.

Dieses Beispiel finden Sie unter:

www.mathworks.de/ltc

\section{The language of technical computing}

